Polytechnic University of Madrid

School of Aeronautical and Space Engineering

STRUCTURAL HEALTH MONITORING IN AERONAUTICAL STRUCTURES BY MEANS OF DISTRIBUTED

FIBRE OPTIC SENSING NETWORKS

Doctoral Thesis

Patricia Fernández Díaz-Maroto

Aeronautical Engineer

2018 

Department of Aerospace Materials and Production

School of Aeronautical and Space Engineering

Polytechnic University of Madrid
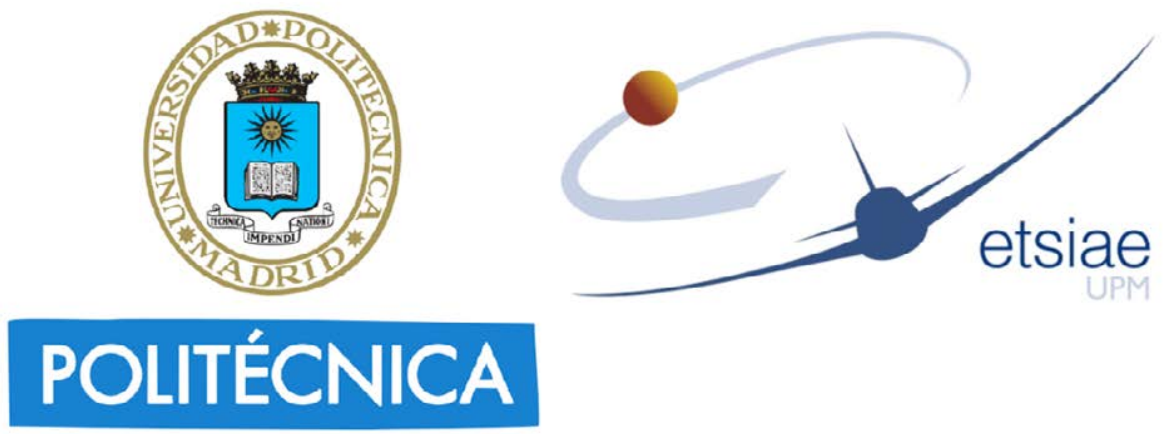

POLITÉCNICA

\title{
STRUCTURAL HEALTH MONITORING IN AERONAUTICAL STRUCTURES BY MEANS OF DISTRIBUTED FIBRE OPTIC SENSING NETWORKS
}

\author{
Author: \\ Patricia Fernández Díaz-Maroto \\ Aeronautical Engineer \\ Supervised by: \\ Jesús Alfredo Güemes Gordo \\ $\mathrm{PhD}$ in Aeronautical Engineering \\ Antonio Fernández López \\ $\mathrm{PhD}$ in Aeronautical Engineering
}





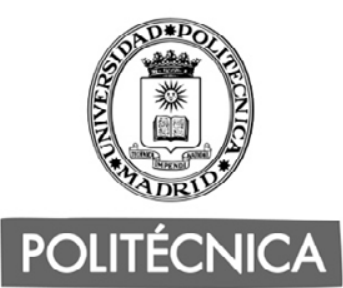

Tribunal nombrado por el Sr. Rector Magfco. de la Universidad Politécnica de

Madrid, el día de 20 .

Presidente

Vocal

Vocal

Vocal

Secretario

Suplente

Realizado el acto de defensa y lectura de la Tesis el día..........de...... de $2 \ldots \ldots$. en la E.T.S.I. / Facultad.

Calificación

EL PRESIDENTE

LOS VOCALES 



\section{ABSTRACT}

Ensuring the reliability of the aeronautical structures throughout its entire operating life has always been one of the most important challenges achieved by complex and thorough aircraft maintenance tasks. Therefore, the development of monitoring techniques to ensure the structural integrity and the ability to predict the structural failure at an early stage would help to improve its reliability. Furthermore, a maintenance plan based on the real aircraft usage leads to significant savings in terms of cost and time.

The major subject of this thesis focuses on the exploitation of the data from distributed measurements of fibre optic sensing networks to detect damage in aeronautical structures. Rayleigh scattering-based sensing technique has been selected to obtain strain and/or temperature distributed measurements. This technique, unlike the rest of the distributed sensing techniques by fibre optic sensors, is the one which enables the best spatial resolution regarding the strain and temperature accuracy. This recent and thriving technique has opened up to new possibilities for structural health monitoring, especially due to its performances in the aerospace field.

In this thesis, the detection of damage through distributed strain measurements has been addressed from two aspects: local and global damage approaches. On the one hand, the local damage detection approach has been demonstrated in different laboratory tests in order to develop and validate all the algorithms necessary to detect and locate damage from the strain measurements. On the other hand, the global damage detection approach has been evidenced for two composite stiffened panels representative of a cockpit fuselage skin for a regional aircraft cabin. Artificial damages have been induced in both panels in order to analyse its growth throughout a fatigue experimental test. Moreover, panels have also been tested under shear loads to carry out the buckling detection and location by means of distributed strain measurements in comparison to conventional sensing techniques.

For this purpose, a novel methodology has been developed based on a non-linear events detection by means of a second derivative analysis of the distributed strain measurements. The application of a Savitzky-Golay filter based on the signal conditioning parameters has become a key to reduce the signal noise generated by the derivatives of the distributed strain measurements. Furthermore, this methodology has also been compared with the so-called "Bifurcation" classic method to detect the buckling onset by comparing discrete strain measurements on both sides of the structure.

Finally, this thesis concludes that this technique offers extensive possibilities for structural health monitoring with very high resolution and allowing the data exploitation in a simple and efficient manner. 


\section{RESUMEN}

Asegurar la fiabilidad de las estructuras aeronáuticas durante toda su vida operativa ha sido siempre uno de los principales retos que se ha ido cumpliendo a través de complejas y minuciosas operaciones de mantenimiento en la aeronave. Por ello, el desarrollo de técnicas de monitorización que aseguren la integridad estructural y sean capaces de predecir un fallo temprano en la estructura, aportaría una mayor fiabilidad de las estructuras basada en el estado real de la misma, a la vez que significaría un importante ahorro en los planes y tareas de mantenimiento de la aeronave.

El tema principal de esta tesis se centra en la explotación de los datos de medida distribuida a través de sensores de fibra óptica para la detección de daño en estructuras aeronáuticas. La técnica de medida distribuida seleccionada ha sido aquella que permite la extracción de datos en deformaciones y/o temperatura a través de la medida de la dispersión de Rayleigh en la fibra óptica. Esta técnica, a diferencia del resto de técnicas de medida distribuida, presenta la mayor resolución espacial con relación a la precisión en la medida de deformaciones y temperatura. Por estos motivos, esta reciente y próspera técnica ha abierto nuevas posibilidades en la monitorización de la salud estructural, y especialmente, debido a sus altas prestaciones, en el campo aeronáutico.

En esta tesis, la detección de daño a través de la medida distribuida en deformaciones se ha llevado a cabo desde dos puntos de vista: detección de daño local y detección de daño global. Por un lado, la detección de daño local se ha demostrado en diferentes ensayos de laboratorio para servir como base de aprendizaje y validar los algoritmos desarrollados para la detección y localización de daño a través de la medida en deformaciones. Por otro lado, la detección de daño global se ha probado sobre dos paneles de material compuesto rigidizado representativo del fuselaje de una cabina de avión regional. Sobre estas estructuras se ha analizado el crecimiento de daños inducidos artificialmente durante un ensayo a fatiga. A continuación, los paneles han sido ensayados a cortadura para llevar a cabo la detección y localización de pandeo a través de la medida distribuida en deformación y comparándolo a su vez con técnicas convencionales de sensorización.

Con esta finalidad se ha desarrollado una nueva metodología basada en la detección de no linealidades a través de la segunda derivada del campo de deformaciones obtenido mediante la técnica de medida distribuida. De igual importancia ha sido la aplicación de un filtro Savitzky-Golay adecuado a los parámetros de condicionamiento de la señal con el objetivo de reducir el ruido generado al obtener las derivadas de la medida. A su vez, esta técnica ha sido comparada con el método clásico de "Bifurcación" para detectar el inicio del pandeo a través de la comparación de medidas de deformación discretas en ambos lados de la estructura.

A través de esta tesis se puede concluir que la medida distribuida ofrece múltiples posibilidades para la monitorización de la salud estructural con muy alta resolución y permitiendo explotar los datos de una manera sencilla y eficiente. 


\section{PUBLICATIONS}

Some ideas and figures have appeared previously in the following publications:

\section{Conferences and Proceedings}

The 8th European Workshop on Structural Health Monitoring 2016, Bilbao, Spain. Strain monitoring on a composite aircraft cabin with fiber optic sensors. Patricia F. Díaz-Maroto, Antonio Fernández, Rosario Fernández, Nicolás Gutiérrez, Fernando Lasagni, J. Alfredo Güemes.

The 8th European Workshop on Structural Health Monitoring 2016, Bilbao, Spain. Free-edge delamination location and growth monitoring with an embedded distributed fiber optic network. Patricia F. Díaz-Maroto, Antonio Fernández, Beatriz Larrañaga, Alfredo Güemes.

The 10th International Workshop on Structural Health Monitoring 2015, Stanford, California. Distributed fibre optic sensors for stiffened skin panels strain monitoring and failure mode detection. Patricia F. Díaz-Maroto, Antonio Fernández-Lopez, Alfredo Güemes.

XI Congreso Nacional de Materiales Compuestos, MATCOMP15, Madrid, Spain. Monitorización de uniones adhesivas con sensores distribuidos de fibra óptica. Patricia F. DíazMaroto, Antonio Fernández-Lopez, Alfredo Güemes.

The 7th International Conference on Composites Testing and Model Identification, Madrid, Spain. Fibre optic distributed sensing: a powerful tool for structural tests. Patricia F. Díaz-Maroto, Antonio Fernández-Lopez, Alfredo Güemes.

The 7th European Workshop on Structural Health Monitoring, Nantes, France. Damage detection in composite structures from fibre optic distributed strain measurements. Alfredo Güemes, Antonio Fernández-Lopez, Patricia Fernandez.

The 12th International Conference on Flow Processing in Composite Materials, Enschede, Netherlands. Monitoring non-isothermal polymerisation and crystallisation of cyclic butylene terephthalate composites manufactured by RTM. I. Ortiz de Mendibil, P. Fernandez, A. Fernández-Lopez, J. Aurrekoetxea, A. Güemes.

\section{Journal Papers}

Patricia F. Díaz-Maroto, A. Fernández-López, J. García-Alonso, M. Iglesias, A. Güemes, Buckling detection of an omega-stiffened aircraft composite panel using distributed fibre optic sensors, Thin-Walled Structures. 132 (2018) 375-384. doi:10.1016/j.tws.2018.08.024.

A. Güemes, A. Fernández-López, P. F. Díaz-Maroto, A. Lozano, J. Sierra-Perez, Structural Health Monitoring in Composite Structures by Fiber-Optic Sensors, Sensors. 18 (2018) 1094. doi:10.3390/s18041094. 
During my $\mathrm{PhD}$, I was able to support my research with two international research stays abroad.

- "XIII Convocatoria de Ayudas del Consejo Social para el Fomento de la Formación y la Internacionalización de Doctorandos para el Curso 2014-2015" by the Universidad Politécnica de Madrid. Structures and Composites Laboratory (from June to September 2015 - 3 months). Stanford University, Stanford, California.

- EM - Euro-Asian Sustainable Energy Development (EASED). Japan Exploration Aerospace Agency and Waseda University (from July 2016 to January 2017 - 6 months). Tokyo, Japan. 


\section{AGRADECIMIENTOS}

Va a ser muy difícil plasmar en pocas líneas el agradecimiento que siento hacia todas aquellas personas que han hecho posible, de una forma u otra, que esta tesis salga adelante.

En primer lugar, me gustaría agradecer al personal del departamento de Materiales y Producción Aeroespacial de la ETSIA donde he desarrollado mi trabajo, y muy en especial a mis tutores, Alfredo y Antonio, que me han dado la oportunidad de crecer y formarme durante este tiempo, por su esfuerzo y dedicación.

A lo largo de estos años he podido compartir momentos inolvidables con la pequeña familia de Composites: Beatriz, Rebeca, Ángel, Renato, Jeromy, Andrés y Martín. A todos vosotros, os deseo lo mejor en el ámbito profesional y todavía más en lo personal. No podría olvidarme tampoco de Mari Carmen, Lucía y Amor, por habernos ayudado siempre que lo hemos necesitado.

Agradecer también a Marisa y Cris, las de siempre, por vuestra paciencia durante este último tramo tan duro y por haberme mostrado todo vuestro apoyo. A Darío, Elena, Laura y Pili, porque juntos despegamos en esta aventura y nos queda todavía mucho por volar. A Marta y Alba, por regalarme alegría y grandes momentos siempre.

A mis compañeros de monitorización, que han vivido muy de cerca mi esfuerzo para cerrar esta etapa. Por sus ánimos, consejos y por escucharme cada mañana. En especial a Jaime, por estar siempre dispuesto a ayudar, y a Pablo, del que tanto he aprendido este último año y que ha confiado en esto hasta el final, gracias. Mucho ánimo P., el próximo tienes que ser tú.

El agradecimiento más difícil de expresar es hacia la persona que ha estado a mi lado en los momentos más duros. A ti, Roser, un infinito GRACIAS por toda la paciencia que has tenido día tras día, por guiarme con tu experiencia y apoyarme incondicionalmente. Sin ti, esta tesis no hubiera sido posible. Ahora te toca a ti hacer de gigante.

Un gracias muy especial a Tere y Juanjo, por vuestro cariño y por hacerme sentir como en casa a vuestro lado.

Por último, agradecer a mi hermana y mi madre el amor y la confianza que depositan en mi cada día. Por enseñarme que con esfuerzo y paciencia todo llega. Sois para mi un ejemplo a seguir y no puedo sentirme más afortunada cuando os tengo cerca. Los próximos agradecimientos los leeremos en Chile, F. Díaz-Maroto. Esta tesis está dedicada a vosotras, las personas más importantes de mi vida. 


\section{Contents}

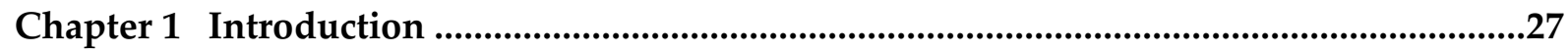

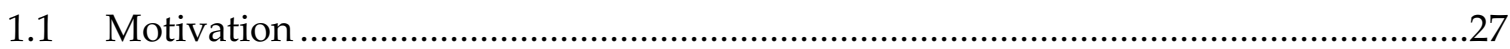

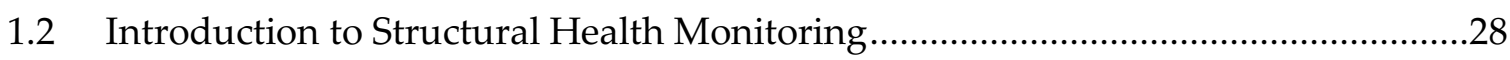

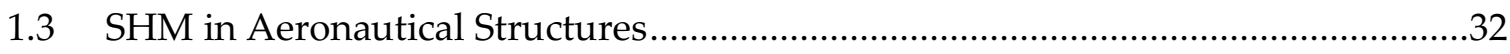

1.3.1 Typical Damages for Aircraft structures...............................................................33

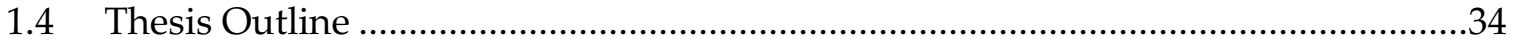

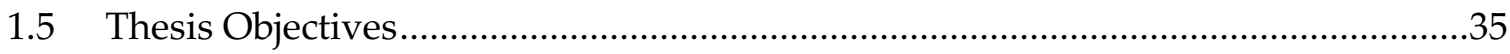

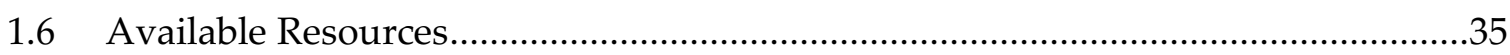

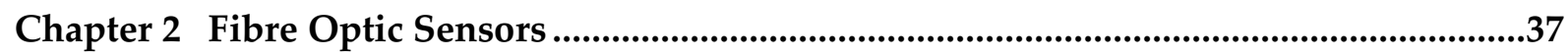

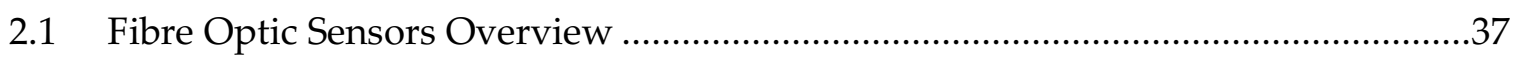

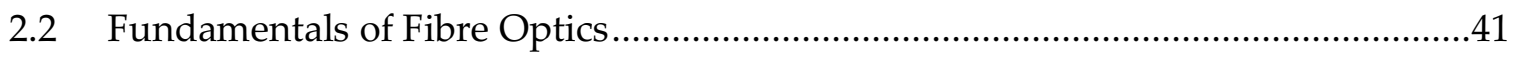

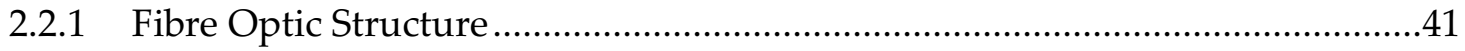

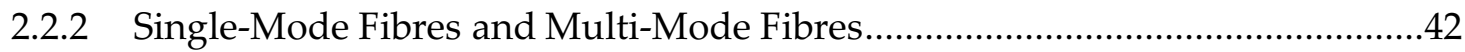

2.2.3 Advantages of Fibre Optic Sensors in SHM for Aircraft Structures ...................44

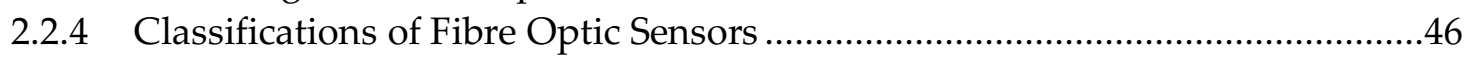

2.3 Optical Reflectometry Techniques.......................................................................

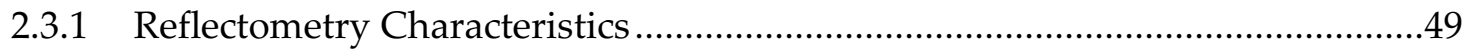

2.3.2 Optical Time Domain Reflectometry …............................................................

2.3.3 Low Coherence Frequency Domain Reflectometry …...................................52

2.3.4 Optical Frequency Domain Reflectometry ….....................................................53

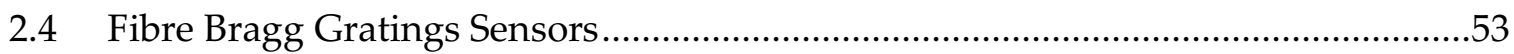

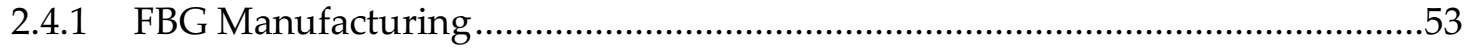

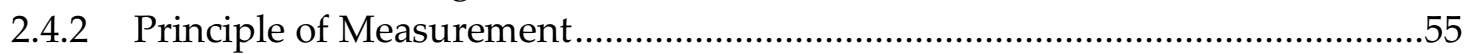

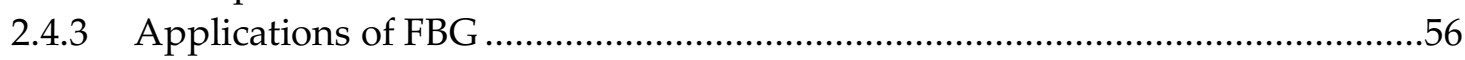

2.4.3.1 Strain Monitoring on a Composite Aircraft Cockpit by FBG.................... 56

2.4.3.2 Strain Monitoring on a Blade by FBG-OFDR technique ............................. 59

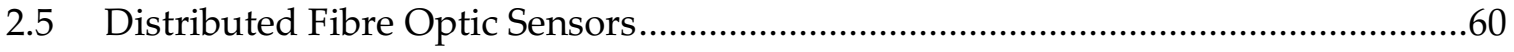

2.5.1 Distributed Sensors Based on Brillouin Scattering ..........................................62 
2.5.1.1 Distributed Brillouin Systems 63

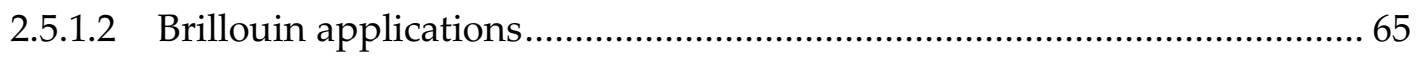

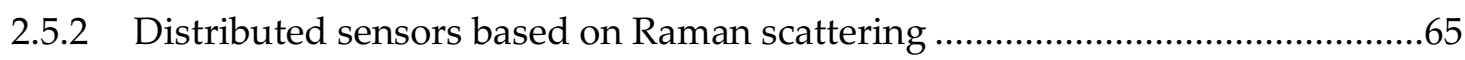

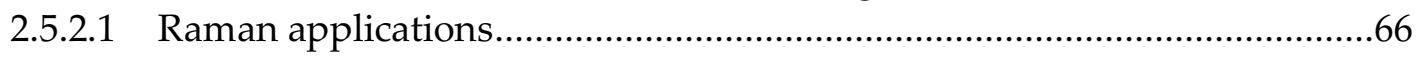

2.5.2.2 Pipe Temperature Monitoring by Raman, OBR and FBG..........................67

2.5.3 Distributed Sensors Based on Rayleigh Scattering ......................................... 70

2.5.3.1 Rayleigh applications........................................................................

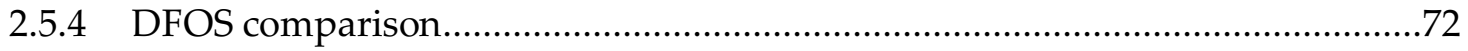

Chapter 3 Measurement Theory of Optical Backscatter Reflectometer..................................74

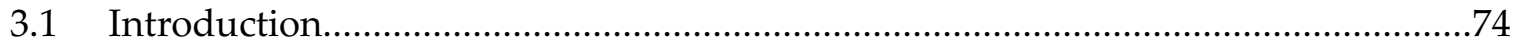

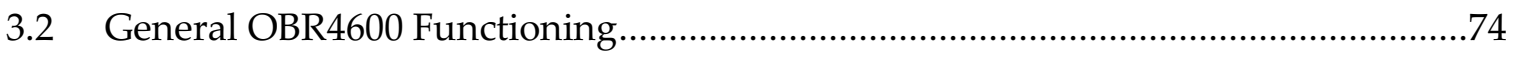

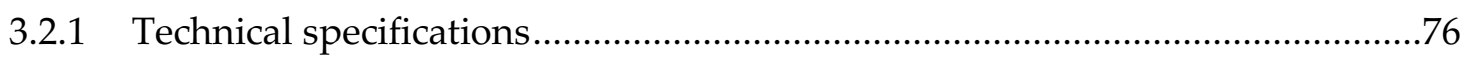

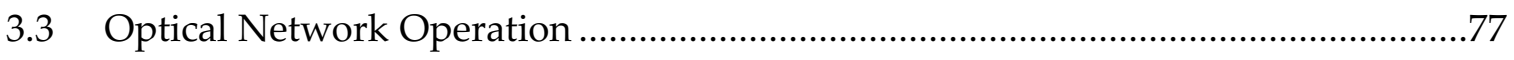

3.4 Interference Pattern Acquisition ..........................................................................

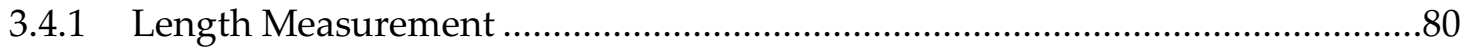

3.5 Group Delay and Polarization Mode Dispersion ......................................................8

3.5.1 Orthogonal Polarization Modes 's' and 'p' .......................................................

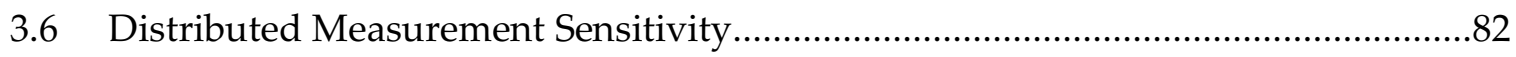

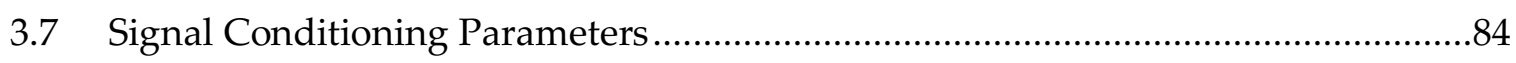

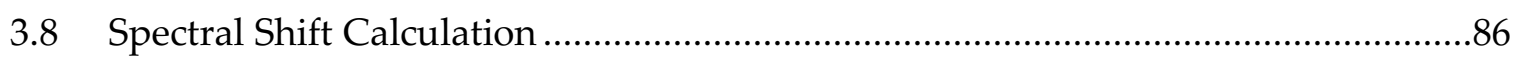

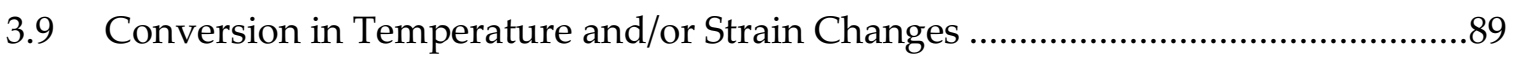

Chapter 4 Damage Detection Methodology...................................................................................91

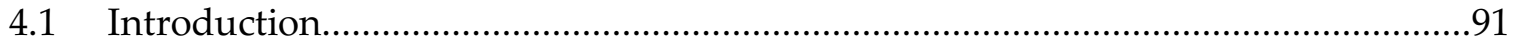

4.2 Experimental Tests and Sensor Network Definition.............................................92

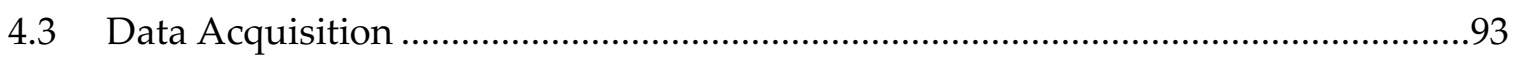

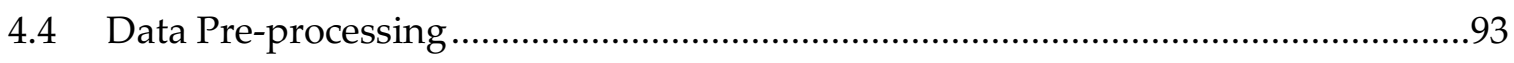

4.4.1 Error Detection and Correction for Distributed Measurement...........................95

4.4.2 Distributed Signal Measurement Smoothing ..................................................96

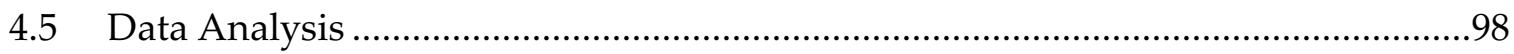

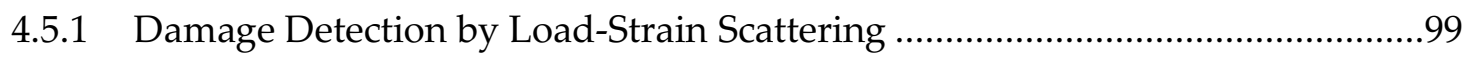

4.5.2 Damage Detection by Second Derivative Analysis..........................................100

4.5.2.1 Example of Second Derivative Analysis...................................................102 


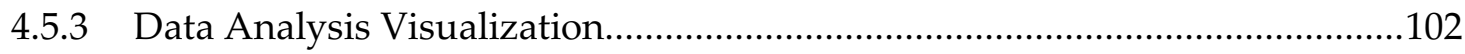

4.5.3.1 Distributed Measurement Maps .............................................................102

Chapter 5 Local Damage Detection Approach...............................................................................104

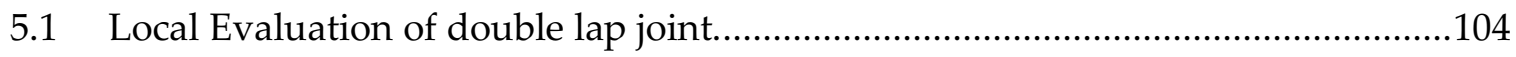

5.1.1 Introduction and Experimental Motivation ….................................................104

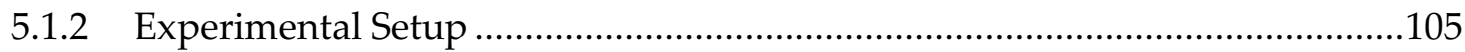

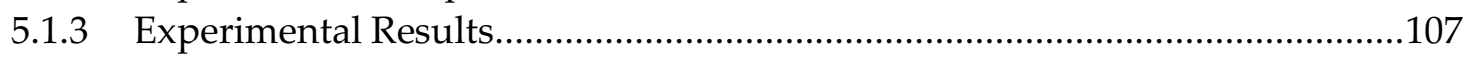

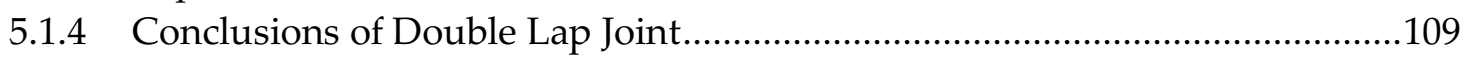

$5.2 \quad$ Free-Edge Delamination Damage ........................................................................... 110

5.2.1 Introduction and Experimental Motivation .................................................110

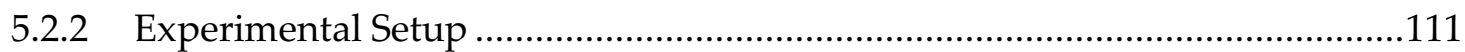

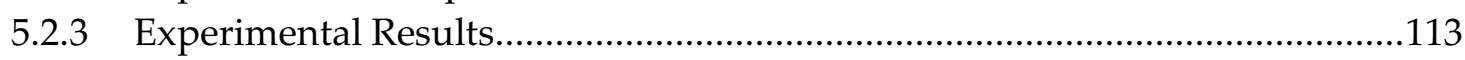

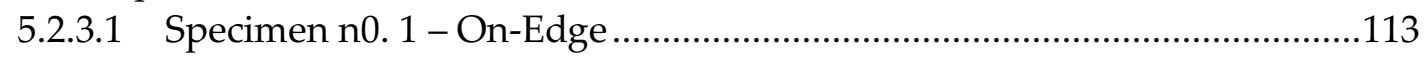

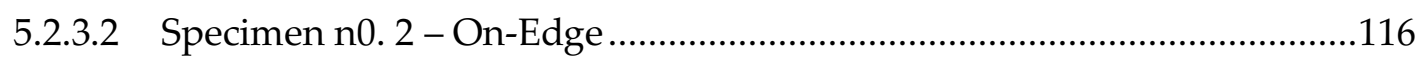

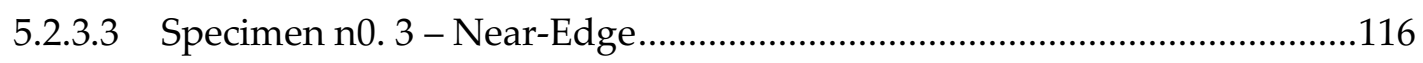

5.2.4 Conclusions of Free-edge test.....................................................................118

Chapter 6 Global Damage Detection Approach ..............................................................119

6.1 Introduction and Experimental Motivation............................................................119

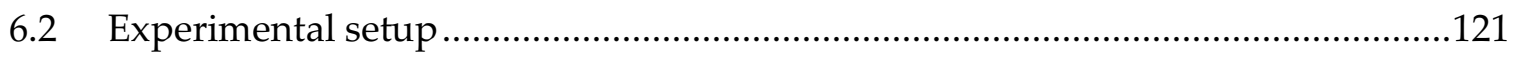

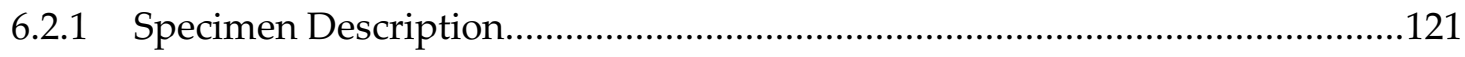

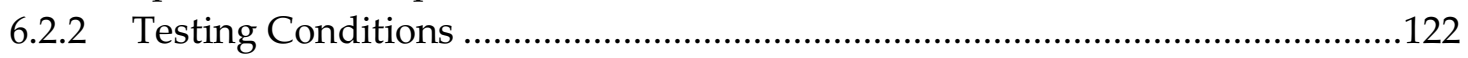

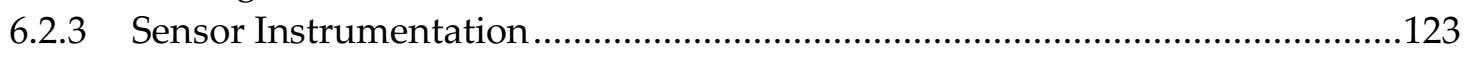

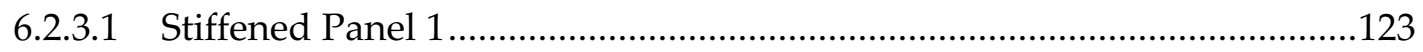

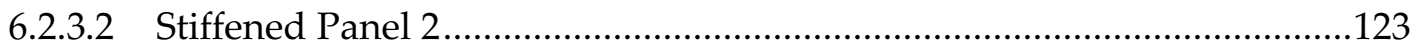

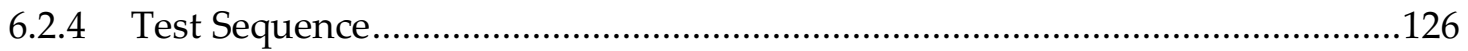

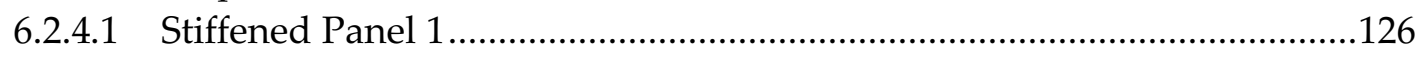

6.2.4.2 Stiffened Panel 2 ....................................................................................12

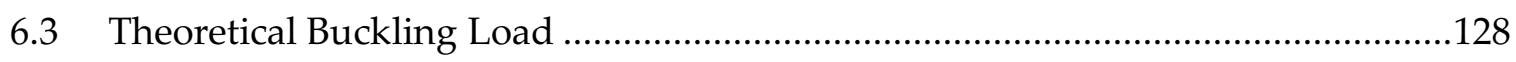

6.4 Temperature Compensation for Distributed Measurement ...................................128

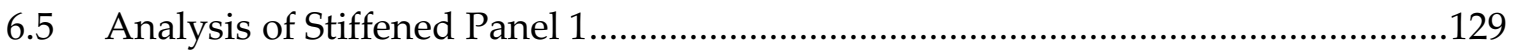

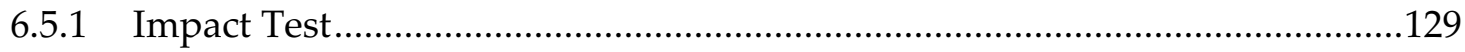

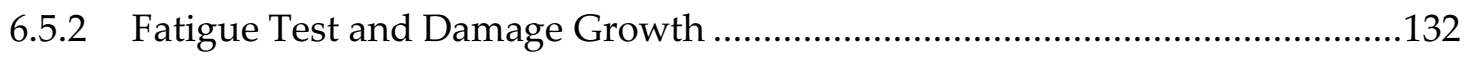

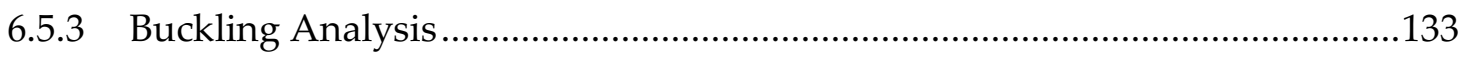

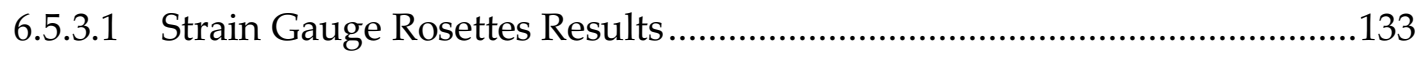

6.5.3.2 Distributed Strain Measurement Results .................................................135 


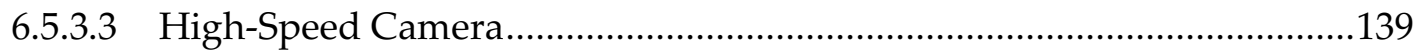

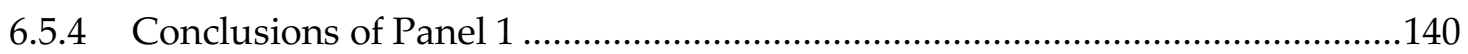

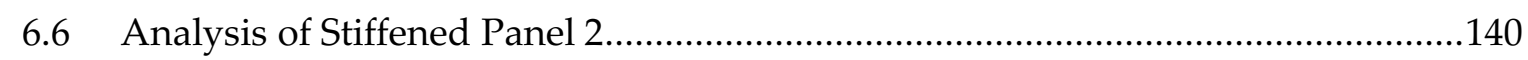

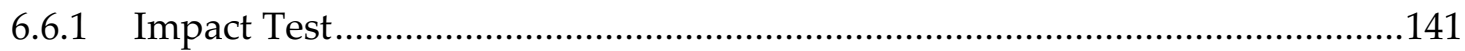

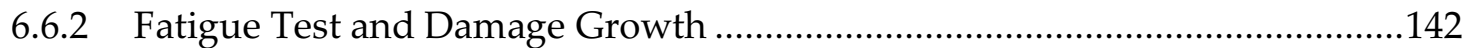

6.6.2.1 Non-Destructive Testing for Fatigue Test ..............................................142

6.6.2.2 Distributed Strain Measurement for Fatigue Test ....................................143

6.6.2.3 Analysis of Damage MUI1 and MUI3 ...................................................147

6.6.2.4 Analysis of Damage MUI2 …..................................................................150

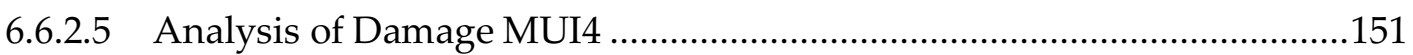

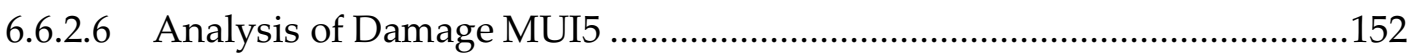

6.6.2.7 Summary of damage growth.................................................................. 153

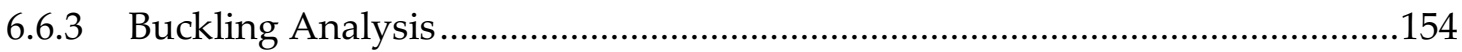

6.6.3.1 Distributed Strain Measurement Results ....................................................155

6.6.3.2 Strain Gauge Rosettes Results ................................................................156

6.6.3.3 Buckling detection by load-strain curves ...............................................158

6.6.3.4 Buckling detection by second derivative approach.................................160

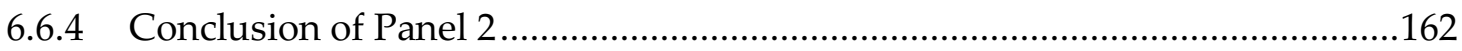

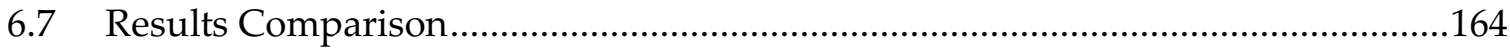

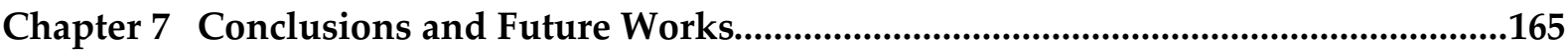

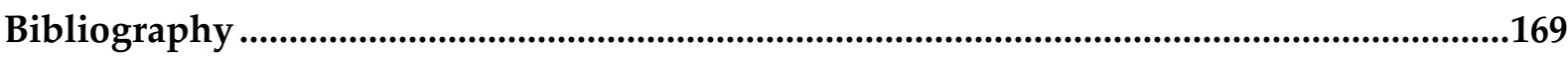

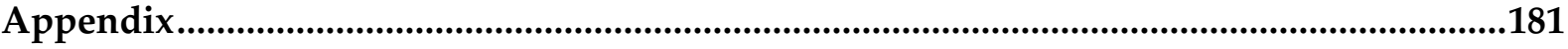




\section{List of Figures}

Figure 1. Examples of FOS application for structural health monitoring in different structures.

Figure 2. (Left) Long time-scale damage in two components. (Right) Short timescale damage for different events in its lifetime.......

Figure 3. Principle and organization of a SHM system (Balageas et al., 2006).................31

Figure 4. Summary of main loads in A/C operation..........................................................

Figure 5. Single-mode interferometer for pressure and temperature measurement based on interferent fringes observation (Hocker, 1979)....................................38

Figure 6. Timeline of main findings and progress in fibre optic sensors..........................40

Figure 7. Main parts of an optical fiber. (a) Multi-mode fibre and (b) single-mode

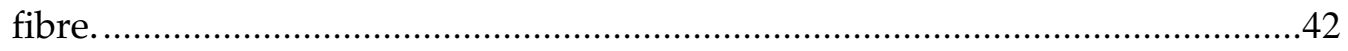

Figure 8. Mode formation with value (Krohn et al., 2014)...........................................43

Figure 9. Attenuation in terms of wavelength (Krohn et al., 2014).................................44

Figure 10. Example of fibre optic embedded in a stiffened composite panel.....................44

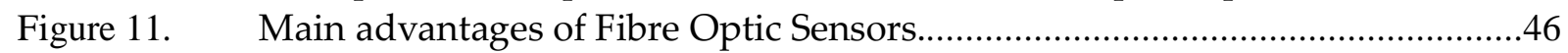

Figure 12. Classification of fibre optic sensors (Di Sante, 2015)......................................47

Figure 13. Classification FOS based on its topology .....................................................48

Figure 14. (Upper) Single point strain and/or temperature measurement. (Lower)

Distributed strain and/or temperature measurement.......................................49

Figure 15. Comparison of reflectometry techniques regarding length and resolution

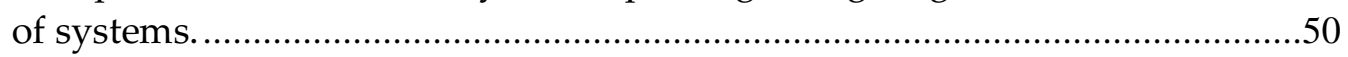

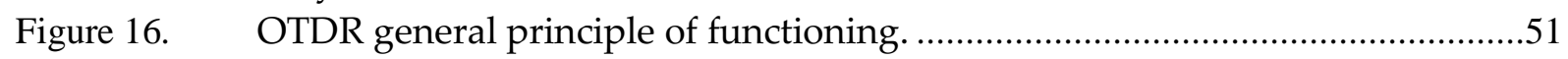

Figure 17. OLCR general schema of functioning ( adapted from Pluciński et al.,

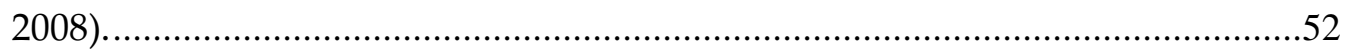

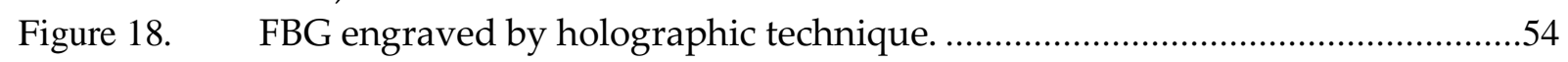

Figure 19. FBG engraved by phase mask and UV laser. ...............................................55

Figure 20. Four fibre optic paths which includes the FBG sensors (black squares)...........57

Figure 21. FBG measurements throughout the structural test...........................................57

Figure 22. Schema of functioning of a FBG-OFDR system (Murayama, Wada, \&

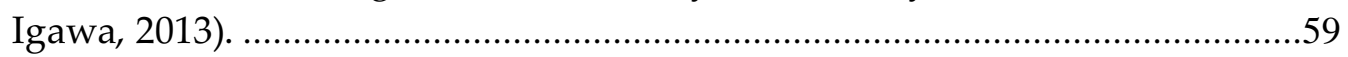

Figure 23. Experimental results for OFDR-FBG validation. (Left) First static test with a hanging load of $113 \mathrm{kN}$. (Right) Second static test with a hanging load of $97.5 \mathrm{kN}$.

Figure 24. Stokes and Anti-Stokes scatterings (adapted from (Fang, Chin, Qu, \& Cai, 2012)).

Figure 25. Light scattering: Raman, Rayleigh and Brillouin. ............................................62

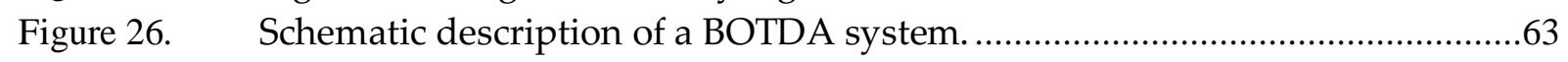


Figure 27. Schematic description of a BOTDR system.

Figure 28. Principle of measurement of a DTS system (Hurtig, Großwig, \& Kühn, 1996)

Figure 29. Setup of experimental distributed temperature measurement.

Figure 30. Single measurement acquired by Halo-DTS. (Left) Stokes and Anti-

Stokes components of Raman scattering.

Figure 31. Distributed temperature measurement by OBR system. The dashed line is the average of the distributed measurement.

Figure 32. Temperature-time histories for the FBGs placed at lower and upper section points, for the Halo-DTS and for the OBR.

Figure 33. Formation of the reflectivity pattern in an optical fibre based on Rayleigh backscattering.

Figure 34. An aluminium coupon under $2 \mathrm{kN}$ and $4 \mathrm{kN}$ of tensile load. The number of spectral shifts can be obtained after cross-correlation against the reference measurement (Patricia F. Díaz-Maroto et al., 2018).

Figure 35 . Commercial Luna OBR-4600 system

Figure 36.

Functioning schema of an OBR.

Figure 37.

Simplified OBR system schema. Three basic components: TLS, DUT and

Mach-Zender interferometer.

Figure 38. Reflection peak in time domain for an instant frequency (Luna

Technologies Inc., 2013).

Figure 39. Differential Group Delay in an optical fibre.

Figure 40. Polarization states in time domain in a real measurement acquired by the OBR. The measurement has been obtained by a moving average of 1000 points.

Figure 41. Experimental setup for the crystallisation monitoring.

Figure 42. Results of the crystallisation experimental test. (a) Temperature, (b) Conversion degree, (c) DSC test, (d) fibre optic losses at different paths. .83

Figure 43. Gauge length and sensor spacing possibilities.

Figure 44. OBR parameters for signal conditioning. (Upper) Total reflectivity measurement for the reference and loaded measurement. (Lower) Specific signal conditioning parameters.

Figure 45. Spectral shift between the reference and the loaded of an OBR measurement. (a) Rayleigh Backscatter Amplitude in frequency domain.........86

Figure 46. Results of the cross-correlation in different fibre sub-sections. .88

Figure 47. Raw data obtained in the cross correlation of the distributed measurement and the cubic spline interpolation of the raw data.

Figure 48. Tasks sequence for each experimental test. ...................................................92

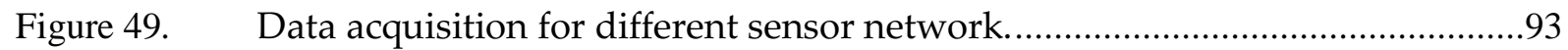

Figure 50. Output file of the OBR system after signal conditioning parameters selection......

Figure 51. OBR pre-processing flowchart.

Figure 52. (a) Optical losses, (b) global signal noise, (c) local signal noise and (d) signal spikes. 
Figure 53. Polynomial order of Savitzky-Golay filter and RMSE in distributed strain approximation (Patricia F. Díaz-Maroto et al., 2018)..

Figure 54. (Left) First derivative of initial strain measurements. (Right) First derivative after Sgolay filter applied.....

Figure 55. Relationship between the original sensing location in the fibre optic length and the local positioning on the structure through the layout matrix.....

Figure 56. (Left) Distributed strain measurements on a composite reinforced panel. (Right) Strain against load for random sensing points (Patricia; F.DíazMaroto et al., 2015).

Figure 57. Example of global buckling detection by the second strain derivative analysis (Patricia F. Díaz-Maroto et al., 2018).

Figure 58. Flow char of the algorithm methodology to perform distributed strain

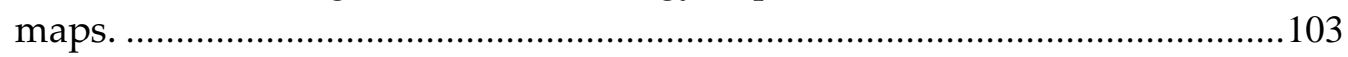

Figure 59. Double lap metallic joint monitored by two optical fibre................................105

Figure 60. (Left) Optical fibre embedded in the aluminium plate. (Right) Ingress/Egress of the optical fibre with additional adhesive.

Figure 61. Double lap joint test held in the MTS machine. Optical losses can be

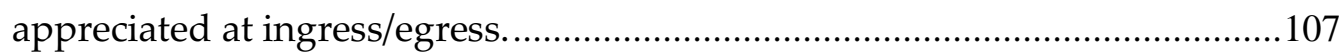

Figure 62. Distributed fibre optic measurement at $3 \mathrm{kN}, 4 \mathrm{kN}$ and $6 \mathrm{kN}$.......................108

Figure 63. Boundary conditions of FEM for the double lap joint and $3 \mathrm{kN}$ of tensile

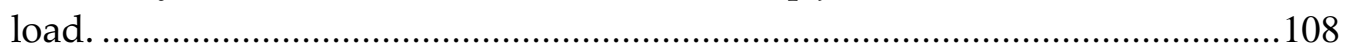

Figure 64. FEM results for the double lap joint under $3 \mathrm{kN}$ of tensile load.....................109

Figure 65. Comparative of FEM results and distributed strain measurement under

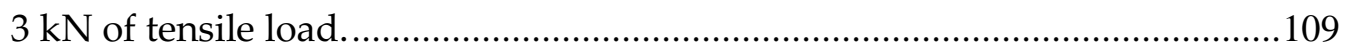

Figure 66. Positioning of polyimide fibre optic during specimen manufacturing...........111

Figure 67. Drop-weight testing machine. (a) On-edge impact. (b) Near-edge impact.....112

Figure 68. Schema of different impact configurations and disposition of fibre optic network bonded close to the near edge made up 6 paths separated $5 \mathrm{~mm}$ each other.

Figure 69. Whole distributed strain measurement from impacts from $1 \mathrm{~J}, 2 \mathrm{~J}, 3 \mathrm{~J}$ and

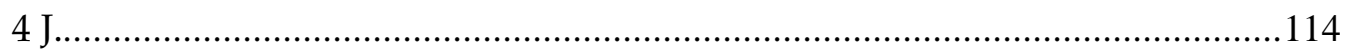

Figure 70. Residual strain evolution after each impact from 2J, 3J and 4J for specimen $n 0.2$ face A. ......................................................................... 114

Figure 71. Residual strain evolution after each impact from 2J, 3J and 4J for specimen $\mathrm{n} 0.1$ face $\mathrm{B}$.

Figure 72. Strain colour map on both faces (A and B).

Figure 73. (Left) Specimen 2 before impact test n0.1. (Right) Specimen n0.1 after 5J of impact energy. Lamina debonding appeared on face A side.

Figure 74. All distributed fibre optic measurement for specimen n0.2.

Figure 75. All distributed fibre optic measurement for specimen n0.3- delamination near edge.

Figure 76. 3D residual strain map for specimen $\mathrm{n} 0.3$

Figure 77. NDT results for the specimen n 0.3 after $6.8 \mathrm{~J}$ of impact energy 118 
Figure 78. Local and global buckling in stiffened panels (Coburn, $\mathrm{Wu}$, \& Weaver, 2014).

Figure 79. (Left) Dimensions of flat side panel with two metallic frames (F1 and F2), two omega stiffeners (S1 and S2), and the shear frame (dark grey) (Patricia F. Díaz-Maroto et al., 2018).

Figure 80. Support conditions, applied load for test specimen and sensors (Patricia

F. Díaz-Maroto et al., 2018).

Figure 81. Detailed figure of the fibre optic sensing network and back-to-back strain gauges rosettes.

Figure 82. The second stiffened composite panel. The two fibre optic networks can be appreciated along with the strain gauges over the panel and the damage area.

Figure 83. Detailed figure of the optical fibre regarding the rosettes location.

Figure 84. Buckling appearance under compressive load. (a) First and (b) second mode.

Figure 85. Strain compensation by temperature increment effect.

Figure 86. Impact locations on the stiffened panel 1. Start and end lines of the fibre optic paths.

Figure 87. All impacts, from impact 1 (top) to 4 (bottom). The damaged area is expressed in millimetres.

Figure 88. Fatigue Test

Figure 89.

Residual strains obtained from the distributed measurement around the impact 1 .

Figure 90. Residual strains obtained from the distributed measurements around the impact 3 .

Figure 91. Load-strain curves from rosettes (a) R1 (b) R2, and R3 (c) (Patricia F. Díaz-Maroto et al., 2018).

Figure 92. Load-strain curves for the $0^{\circ}$ and $45^{\circ}$ of the rosettes R1, R2 and R3. 135

Figure 93. Full distributed strain measurement of the fibre optic line (Patricia F. Díaz-Maroto et al., 2018).

Figure 94. All distributed measurement paths in 3D for $-60 \mathrm{kN},-120 \mathrm{kN}$ and -195 kN (Patricia F. Díaz-Maroto et al., 2018).

Figure 95. Distributed strain measurements from paths 10, 11, 12, and 13. Nonlinear behaviours of each path are highlighted with a red square (Patricia F. Díaz-Maroto et al., 2018).

Figure 96. (Left) First strain field for Path 11. (Right) Second derivative (Patricia F. Díaz-Maroto et al., 2018).

Figure 97. Path 4 distributed strain measurement (left), first derivative (centre), and second derivative (right). (Patricia F. Díaz-Maroto et al., 2018) 138

Figure 98. Summary of distributed strain measurement results (stiffened side). (a) Contour of the second derivative strain map. (b) Detailed buckling appearance of bay 4 from -180 to $-190 \mathrm{kN}$. (c) Strain map at ultimate load (Patricia F. Díaz-Maroto et al., 2018). 
Figure 99. High-speed camera photographs of the stiffened panel at $-195 \mathrm{kN}$ (Patricia F. Díaz-Maroto et al., 2018)................................................................140

Figure 100. Impact Barrel (Courtesy of University Carlos III of Madrid)...........................141

Figure 101. Damages and impact location over the stiffened panel 2 ...............................142

Figure 102. Correlation between path 10 and 11 for tensile test at 45000 cycles. ...............144

Figure 103. Correlation between path 10 and 11 for compressive test at 45000 cycles......144

Figure 104. Strain colour map under $-120 \mathrm{kN}$ of compressive load (a) and $120 \mathrm{kN}$ of tensile load (b) after 45000 cycles.

Figure 105. Comparison of the total of distributed measurements recorded at 45000 , 90000,135000 and 180000 cycles under $-40 \mathrm{kN},-80 \mathrm{kN}$ and $-120 \mathrm{kN}$ of compressive loads.

Figure 106. Comparison of the total of distributed measurements recorded at 45000 , 90000, 135000 and 180000 cycles under $40 \mathrm{kN}, 80 \mathrm{kN}$ and $120 \mathrm{kN}$ of tensile loads.

Figure 107. Distributed measurement around damage MUI1 analysed by path 10, 11 and 12.

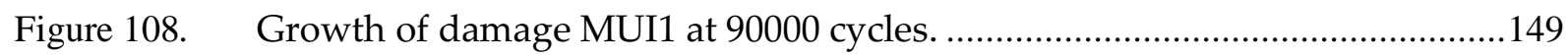

Figure 109. Strain reading at $0,45 \mathrm{~K}, 90 \mathrm{~K}, 135 \mathrm{~K}$ and $180 \mathrm{~K}$ cycles for rosette 4 and $5 \ldots \ldots . .149$

Figure 110. Distributed measurement around damage MUI2 analysed by path 6, 7 and 8.

Figure 111. Growth of damage MUI2 at 38243 cycles.

Figure 112. Distributed measurement around damage MUI4 analysed by path 17, 18 and 19.

Figure 113. Distributed measurement around damage MUI4 analysed by path 3, 4 and 5.

Figure 114. Distributed map of residual strain during fatigue test.

Figure 115. Complete distributed measurement under compressive load for buckling test acquired by the fibre optic channel 1 .....

Figure 116. Complete distributed measurement under compressive load for buckling test acquired by the fibre optic channel 2.........................................156

Figure 117. Full-field strain for $-235 \mathrm{kN}$ of compressive load.

Figure 118. Comparison of compressive buckling test between rosettes and distributed measurement.

Figure 119. Rosettes strain gauge comparison at $0^{\circ}$ for buckling test under compressive load.

Figure 120. Rosettes strain gauge comparison at $0^{\circ}$ for buckling test under tensile load.

Figure 121. (Left) Distributed strain measurement recorded by the OBR for path 20 under compressive load. (Right) Bifurcation of load-strain curves at three different lengths.

Figure 122. (Left) Distributed strain measurement recorded by the OBR for path 4 under compressive load. (Right) Bifurcation of load-strain curves at three different lengths. 
Figure 123. (Left) Distributed strain measurement recorded by the OBR for path 4 under tensile load. (Right) Bifurcation of load-strain curves at three different lengths

Figure 124. Results of the second derivative map for buckling detection under -235 $\mathrm{kN}$ of compressive load.

Figure 125. Distributed strain measurement for buckling test under compressive load on paths 2, 3, 4 and 5 .

Figure 126. Results of the second derivative map for buckling detection under 300 $\mathrm{kN}$ of tensile load.

Figure 127. Comparison between compressive and tensile buckling test around damage MUI1.

Figure 128. Results of the second derivative analysis for the stiffened panel 1. 183

Figure 129. Whole distributed measurement for the buckling test under compressive load for the stiffened panel 2.

Figure 130. Whole distributed measurement for the buckling test under tensile load for the stiffened panel 2 


\section{List of Tables}

Table 1. Comparison of the strain read-out for the FBG and strain gauge sensors

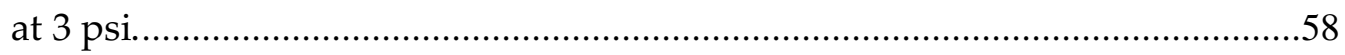

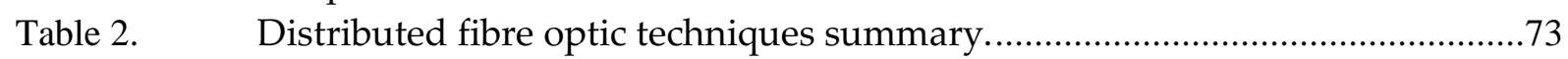

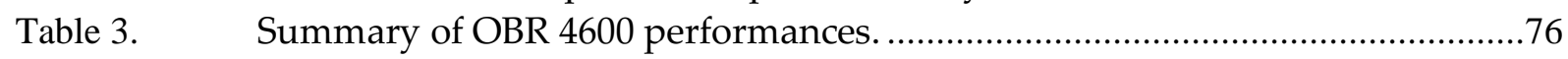

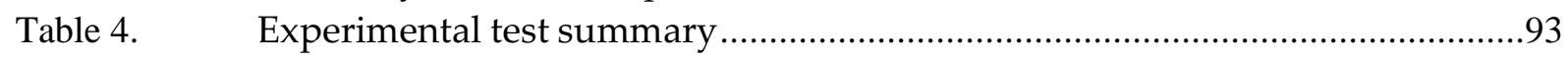

Table 5. Common errors and issues for OBR distributed measurements. .......................96

Table 6. Properties of aluminium plates and adhesive used for the experimental

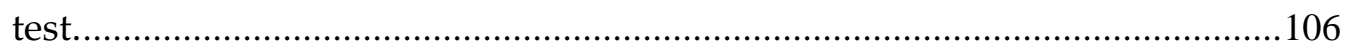

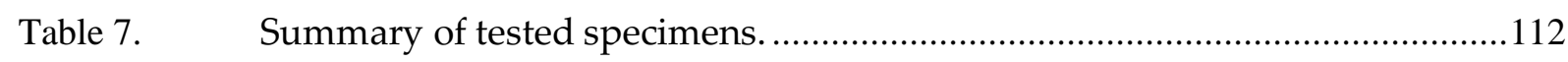

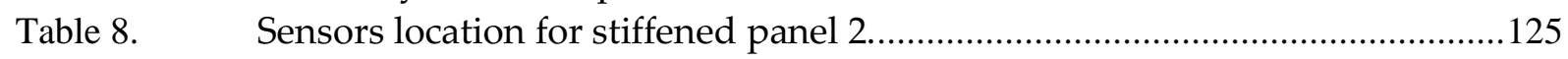

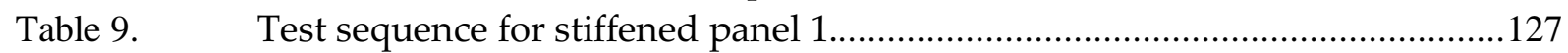

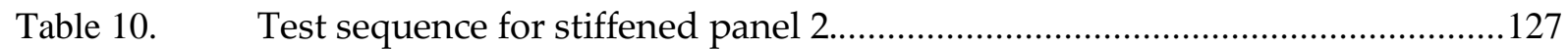

Table 11. Buckling critical load for tensile and compressive test....................................128

Table 12. Specification of impacts for stiffened panel 1..............................................130

Table 13. Residual strains of strain gauge rosettes after buckling test. ..........................135

Table 14. Specification of impacts for stiffened panel 2 ...............................................141

Table 15. Non-Destructive Testing summary for damage growth during fatigue

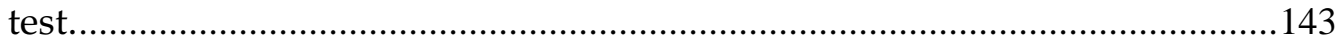




\section{List of Abbreviations}

$\begin{array}{ll}\text { BOCDA } & \text { Brillouin Optical Correlation Domain Analysis } \\ \text { BOFDA } & \text { Brillouin Optical Frequency Domain Analysis } \\ \text { BOTDA } & \text { Brillouin Optical Time Domain } \\ \text { BOTDR } & \text { Brillouin Optical Time Domain Reflectometry } \\ \text { c } & \text { Vacuum light velocity } \\ \text { CAMID } & \text { Compression Air Mobile Impact Device } \\ \text { CBM } & \text { Condition Based Maintenance } \\ \text { CCMSS } & \text { Centre of Composite Materials and Smart Structures } \\ \text { CFRP } & \text { Carbon Fibre Reinforced Polymer } \\ \text { CM } & \text { Condition Monitoring } \\ \text { CW } & \text { Continuous Wave } \\ \text { DAQ } & \text { Data Acquisition } \\ \text { DFOS } & \text { Distributed Fibre Optic Sensor } \\ \text { DGD } & \text { Differential Group Delay } \\ \text { DIC } & \text { Digital Image Correlation } \\ \text { DP } & \text { Damage Prognosis } \\ \text { DSC } & \text { Differential scanning calorimetry } \\ \text { DSF } & \text { Damage Sensitive Feature } \\ \text { DTS } & \text { Distributed Temperature Sensors } \\ \text { DUT } & \text { Device Under Test } \\ \text { EDC } & \text { Error Detection and Correction } \\ \text { FBG } & \text { Fibre Bragg Grating } \\ \text { FC/APC } & \text { Fibre Connector / Angle Physical Contact } \\ \text { FEM } & \text { Finite Element Model } \\ \text { FFT } & \text { Fast Fourier Transform } \\ \text { FMCW } & \text { Frequency Modulated Continuous Wave } \\ \text { FO } & \text { Fibre Optic } \\ \text { FOS } & \text { Fibre Optic Sensors } \\ \text { GD } & \text { Group Delay } \\ & \end{array}$




\begin{tabular}{|c|c|}
\hline GL & Gauge Length \\
\hline HUMS & Health and Usage Monitoring \\
\hline LM & Limit Load \\
\hline MMF & Multi-Mode Fibre \\
\hline NA & Numerical Aperture \\
\hline NDT & Non-Destructive Testing \\
\hline OBR & Optical Backscatter Reflectometer \\
\hline OFDR & Optical Frequency Domain Reflectometry \\
\hline OLCR & Optical Low Coherence Reflectometry \\
\hline OTDR & Optical Time Domain Reflectometry \\
\hline PMD & Polarization Mode Dispersion \\
\hline RBS & Rayleigh Backscattering \\
\hline RMSE & Root Mean Square Error \\
\hline RTM & Resin Transfer Moulding \\
\hline SBS & Stimulated Brillouin Scattering \\
\hline SCP & Signal Conditioning Parameters \\
\hline SG & Strain gauge \\
\hline SHM & Structural Health Monitoring \\
\hline SHMS & Structural Health Monitoring System \\
\hline SMF & Single-Mode Fibre \\
\hline SPC & Statistical Process Control \\
\hline SRS & Stimulated Raman Scattering \\
\hline SWI & Swept Wavelength Interferometry \\
\hline TLS & Tuneable Laser Source \\
\hline UMF & Usage Monitoring Function \\
\hline UL & Ultimate Load \\
\hline UV & Ultraviolet \\
\hline
\end{tabular}




\section{Chapter 1 INTRODUCTION}

This chapter provides a general overview of the motivation for the research topic of this thesis. A brief state of the art for structural health monitoring in aeronautical structures is also presented from a fibre optic sensor's perspective. Finally, the outline, objectives and available resources for this thesis are presented through this introductory chapter.

\subsection{Motivation}

As is stated in the title of this thesis, the major aim of this work is to detect damages in representative aeronautical structures by means of distributed strain measurements acquired with help of fibre optic sensors (FOS). Different methodologies have been developed to achieve this goal, tested in laboratory specimens and finally proved in a real composite structure which belongs to a regional aircraft.

The motivation to carry out this thesis revolves around the need to ensure the reliability of aeronautical structures whose failure triggers catastrophic consequences. Thereby, structural health monitoring field developed in this context, not only for aeronautical structures, but also for a large variety of frameworks. The increase and development of different sensing techniques which allow to measure nearly every parameter conceivable for the structures, motivated the growth of the structural health monitoring field.

In the quest to achieve more powerful techniques for structural health monitoring, distributed fibre optic sensors have been breaking through as a one the most promising technologies. Particularly, among the three key techniques to achieve distributed measurements from fibre optics, Rayleigh technique has already proved to be the most suitable to monitor aircraft structures due to its high performances in terms of spatial resolution and measurement accuracy.

Since its appearance, distributed fibre optic sensors have been widely used and tested in different applications. Therefore, the capability to obtain distributed temperature measurements for large distances has strongly burst in the field of pipes thermal control in order to detect leaks. Most innovative companies have already included this monitoring system for more than 20 years (Vurpillot, Inaudi, \& Ducret, 1996). In the field of the large 
civil structures, bridge deformation monitoring has been always a huge challenge to ensure its feasibility. Thanks to the distributed strain measurement through fibre optics this goal has been successfully achieved. Regarding the aeronautical field, fibre optics have become incorporated into different domains. Then, one of the most common applications lies in the structural tests where conventional strain gauge sensors have been replaced by FOS due to its easier installation and integration. Structures such as a wind turbine blade of $40 \mathrm{~m}$ length have been successfully monitored under critical buckling loads (Guemes, Fernandez-Lopez, \& Soller, 2010). Furthermore, the FOS technology is breaking through into the experimental flight test domain to detect impacts, stress distribution, temperature changes and strain flight profile. Along these lines, structural health monitoring by FOS has potential growth as a particular field whose sensor data is evaluated to detect damages and material changes that compromise the performance of the structures.
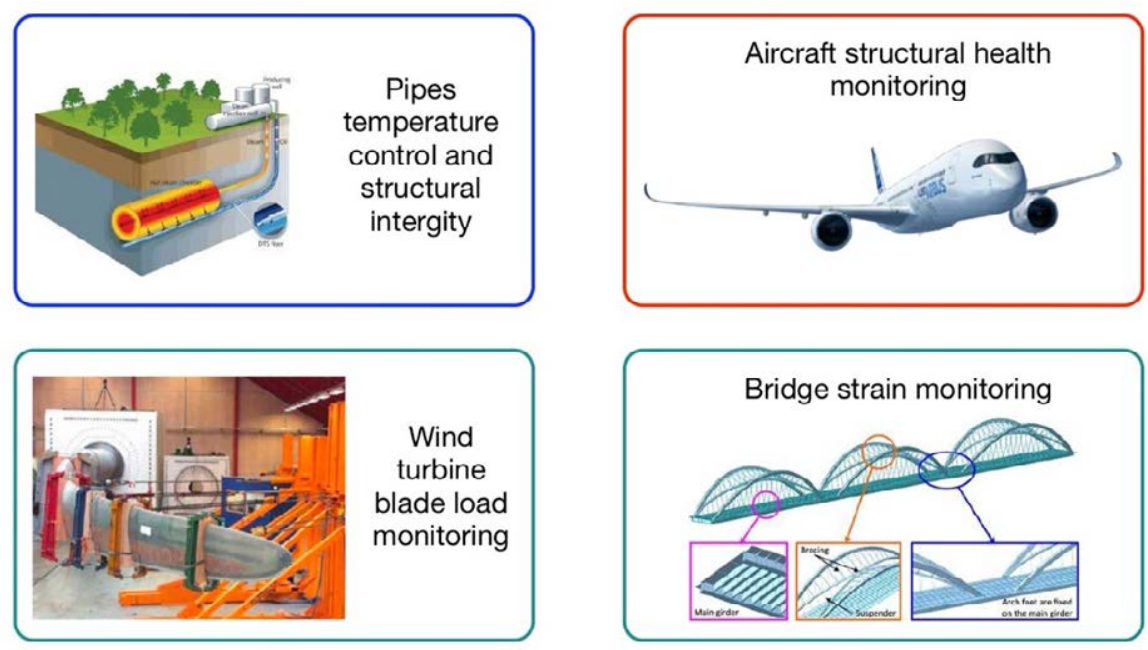

Figure 1. Examples of FOS application for structural health monitoring in different structures.

One of the main challenges to spread the use of FOS for damage detection is focused on the development of algorithms which enable the correlation of damage appearance with the measurand data acquired by the sensing network. In this thesis, the distributed fibre optic sensing (DFOS) exploited the full-field strain of the structures to detect and locate the presence of damage.

\subsection{Introduction to Structural Health Monitoring}

All engineering structures are continuously degraded from the moment they are in service. There are several harsh environments which can contribute to this degradation: humidity, corrosion, overload, fatigue, impact.... For that reason, the maintenance and the intervals of inspection of these structures have always been essential to ensure the life safety and also, to provide economic benefits. In general, most of the structures are under periodic maintenance tasks which include interval of inspection and, in case of presence of damage, corrective actions can be carried out if applicable. Furthermore, the performance of preventive actions so as to avoid failure at an early stage, are also commonly included in the maintenance plan. 
In the case of civil engineering infrastructures, such as buildings, unless they are located in areas of natural disasters, it is unusual to encounter special checks that are not during regular period of inspection. Other civil infrastructures, such as bridges, have come to increase their inspections and join efforts to keep these structures safe life. In the case of power plants, not only are regular inspections carried out, but also additional maintenance tasks have since been defined to prevent failures and avoid potential risks.

Regarding aeronautical structures, both maintenance and inspection have become increasingly challenging due to the complex design of the components that shape the structure as well as the operational mode of the aircrafts. Moreover, the kind of failures that can be encountered in an aircraft come from a wide variety of sources and their detection and characterization becomes very assorted (Wiesław Ostachowicz \& Güemes, 2013). Accidental damages are a very common type of failure in aeronautical structures which may occur, for example, during maintenance operations. Whereas some of the damages can be appreciated at a glance, other structures can host internal damages which are difficult to detect, such as fibre and matrix breakage or delamination. Another problem to deal with under periodic maintenance is when these damages occur during two intervals of inspection since they can culminate into the catastrophic failure of the structure. The concept of condition-based maintenance was developed in this context. In this maintenance strategy, a sensing system on the structure informs the user of the presence of damage in order to take the necessary corrective actions.

Before continuing, some definitions have already appeared in these paragraphs and they need to be clearly described within the context of damage detection in aeronautical structures. These are the terms of structural health monitoring, damage and failure of the structures.

In order of complexity, the definition of damage is the first that has to be settled. Hereafter, damage is associated with a change introduced in a specific structure which involves deficiency in the host material and/or in the geometry properties. In general terms, the presence of damage affects the current structural performance. Hence, in order to identify and detect damage it is necessary to represent a baseline or reference state (normally undamaged) from which to establish a comparison. Then, the term of failure is used when, under different loading states, the damage increases, and the structure or system fails as a consequence of it. At that point, it can be said that structural failure has been reached and the structure cannot operate normality.

In long time-scale terms, damage can also be accumulated over the operational time leading to the failure of the structure, e.g. this is the case of damages caused by fatigue or corrosion. Nevertheless, in shorter time-scale terms, different events over the entire operational lifetime can occur, which can undergo high local damage. Figure 2 illustrates these two sorts of damage appearances that can lead to failure of the structure: by accumulating damage or by isolated events throughout the operational lifetime of the structure. 
Long-time Scale Damage

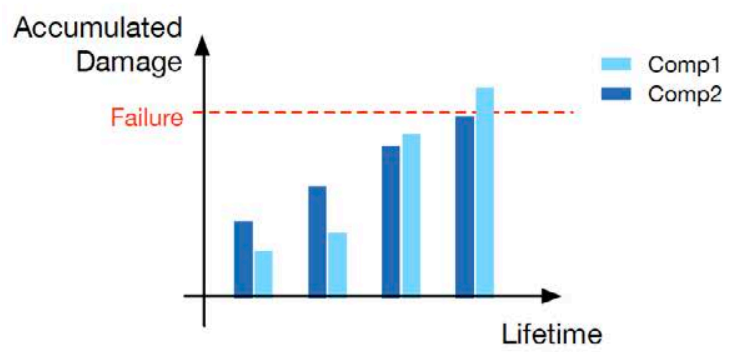

Short-time Scale Damage

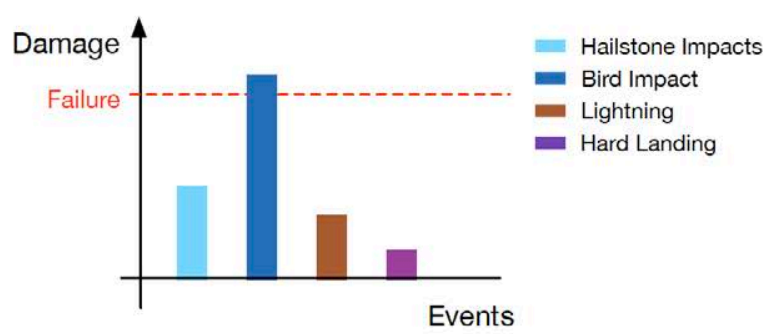

Figure 2. (Left) Long time-scale damage in two components. (Right) Short time-scale damage for different events in its lifetime.

Once the definition of damage and failure has been clarified, the definition of structural health monitoring (SHM) can be addressed. Thereby, the SHM term has had several definitions from different authors in manifold fields of application. Here, the SHM approach is taken from Farrar \& Worden, (2007) which refer to SHM as a "process of implementing a damage strategy for aerospace, civil and mechanical engineering infrastructure". In this way, the sensors installed in the structure are able to extract damage-sensitive features in order to perform an analysis from which the state of the structure over a period of time can be determined. Hence, in this thesis distributed fibre optic acts as a sensor to extract the strain field of the structures which will be summited to external loading factors. Then, the signals acquired by the sensing network need to be processed to identify the presence of damage.

It should be pointed out that a SHM system is cost-effective when it is able to detect damage at an early stage. Hence, a corrective action can be performed before achieving the failure of the structure. Otherwise, the development of the SHM system can become costly and unnecessary when no-preventive task can be performed to avoid the failure of the structure.

Overall, the installation of a SHM system also involves the sensor integration on the structure, the data transmission and processing. Figure 3 is an attempt to schematize the organization of a whole SHM system (Balageas, Fritzen, \& Güemes, 2006). In this way, the existence of damage produces a physical phenomenon which has to be measurable by the sensors installed into the structure. Then, these sensors perform signals that are transmitted to the acquisition system. On the other hand, other usage parameters are measuring simultaneously and stored in the usage monitoring function (UMF). In order to evaluate the residual life of the structure, i.e. determine the prognosis, as well as its health state, data from SHM and UMF systems are combined. Same functioning schema for different structures can provide the Health Management of the full system (aircraft, power plan, bridge...). 


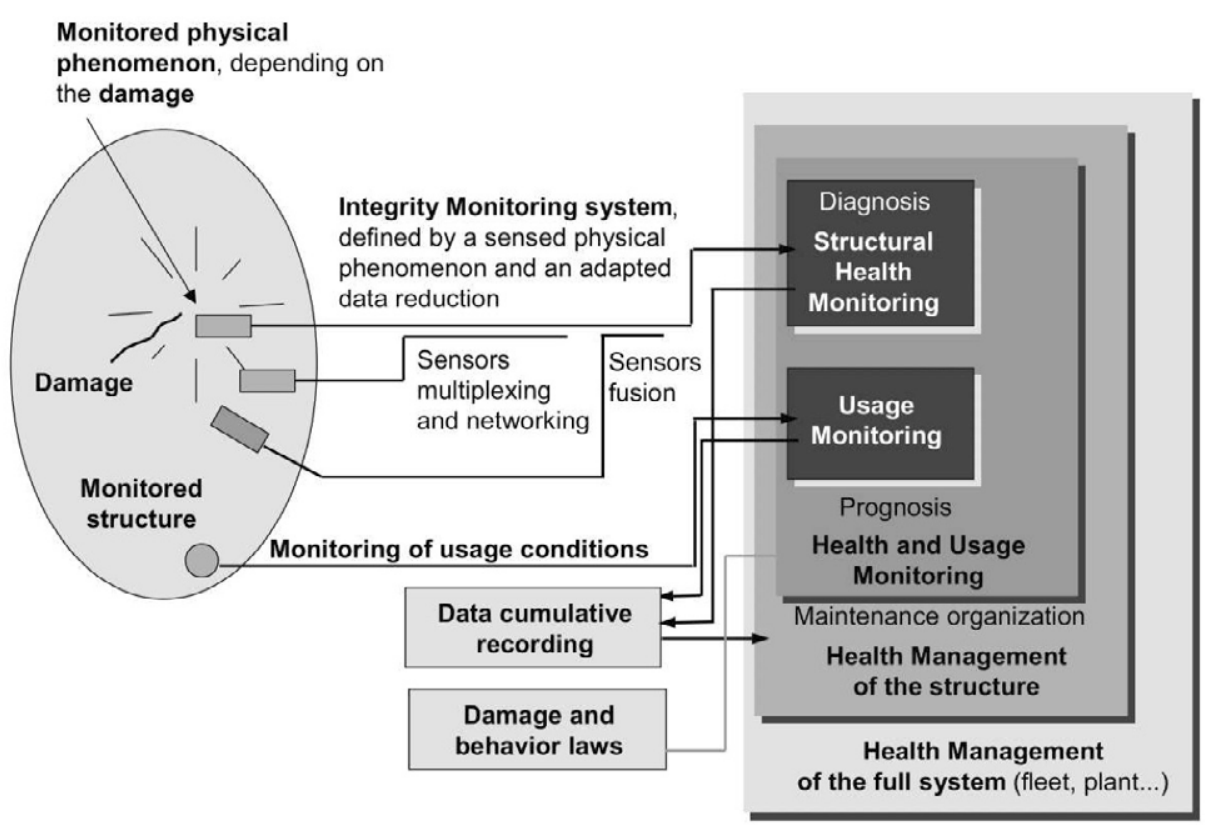

Figure 3. Principle and organization of a SHM system (Balageas et al., 2006).

From a practical point of view, the SHM strategy can be considered as a technique which is divided into four-main levels as described by Rytter (1993):

- Detection, the methodology or strategy developed for health monitoring in a specific structure detects the presence of an anomaly or damage.

- Location, the presence of the damage can be accurately located over the structure.

- Assessment, the severity and damage size are evaluated.

- Prognosis, the safety and reliably of the structure is analysed.

In this thesis, the two first steps are analysed in the results of local damage approach by distributed strain measurements. The detection and location of the damage are two steps that are connected in the case of the distributed measurements, as long as the position of the fibre is known, the presence of the damage is straightforward correlated. Moreover, the assessment level of the damage is also evaluated in the presentation of the global approach.

Not only is SHM a key field for monitoring and assessing damage, but also other disciplines should be mentioned that are relevant in damage evaluation (Worden \& Dulieu-Barton, 2004) and have developed along with SHM.

- Condition Monitoring (CM). This field addresses the detection of damage in rotating and reciprocating machinery such as used in manufacturing (Wiesław Ostachowicz \& Güemes, 2013). Moreover, CM technique is often vibration-based to detect changes in time, frequency or modal domains which indicates the presence of damage (Carden \& Fanning, 2004). 
- Non-Destructive Techniques (NDT). This strategy is usually performed out of line when the damage has already been located. Typical non-destructive techniques such as ultrasounds, thermography and shearography, are used to classify and evaluate the severity of the damages. These techniques aim to interrogate the structure in a non-intrusive manner in order to inform about the current structural state.

- Health and Usage Monitoring (HUMS). This discipline collects the necessary data of loads to calculate and modify the remaining life of the structure. These system's so-called HUMS were successfully implemented in helicopters since the 70s. (Cronkhite, Dickson, Martin, \& Collingwood, 1998).

- Statistical Process Control (SPC). This technique is based on a statistical procedure to detect the presence of damage. In the same manner that occurs with $\mathrm{CM}$ discipline, SPC is well-stablished for rotating machinery and vibration-based (Fugate, Sohn, \& Farrar, 2001).

- Damage Prognosis (DP). As it was already presented as a SHM level, once damage has been detected and located the calculation of the remaining life of the structure is analysed through this discipline.

\subsection{SHM in Aeronautical Structures}

As formerly mentioned in the introduction, aeronautical structures have a substantial number of SHM applications. Unlike other domains, these structures are subject to a wide range of operational environments, some of which are the harshest in terms of structural loads and hazard environments (Himelblau, Manning, Kern, \& Piersol, 2001).

In addition, nowadays, modern aircraft structures have been breaking through in the design optimization and lightweight structures in order to achieve a more cost-effective design. In most cases, these performances make even more necessary the development of a monitoring system which can still offer reliable and quality structures. However, nowadays a very restrictive airworthiness directives limit the use of SHM systems.

The selected system should consider the following aspects to ensure the suitability of the SHM system:

- The type of structure to be monitored. This aspect encompasses not only the structural parts which are integrated, but also the materials and their specific properties.

- The types of damage that the structure is prone to suffer and how they affect the structural integrity.

- The measurand parameter of the structure that is sensitive for a specific type of damage. This is also called damage sensitive feature (DSF).

- How the damage sensitive feature is affected by the damage. 
Once the SHM system is selected according to the criteria above, the technique or methodology for damage detection can be addressed. According to Wieslaw Ostachowicz, Malinowski, \& Wandowski (2013), the main SHM techniques can be classified into:

- Vibration based methods.

- Guided waves-based methods.

- Optical fibre-based methods.

- Acoustic emission-based methods.

- Differential pressures-based methods.

- Electromagnetic fields-based methods.

In this thesis optical fibre-based methods have been adopted for the detection of damage in different representative aeronautical structures.

\subsubsection{Typical Damages for Aircraft structures}

Based on the different structural parts of an aircraft, varied damage can be observed. As was formerly defined, damage is considered to be a change in the host material and/or in the geometry properties which affects the current performance of the structure. As can be seen in Figure 4, the most common damages are classified and highlighted depending on the structural part of the aircraft (Boller \& Staszewski, 2004). Hence, impacts are mostly found in the nose of the aircraft or/and in the leading edge and the tail plane. They can be originated by different sources such as hails, birds or grave. Moreover, the upper wing skin is under compression which is prone to undergo unstable and non-linear behaviours. Finally, the cabin pressurization involves hoop stress.

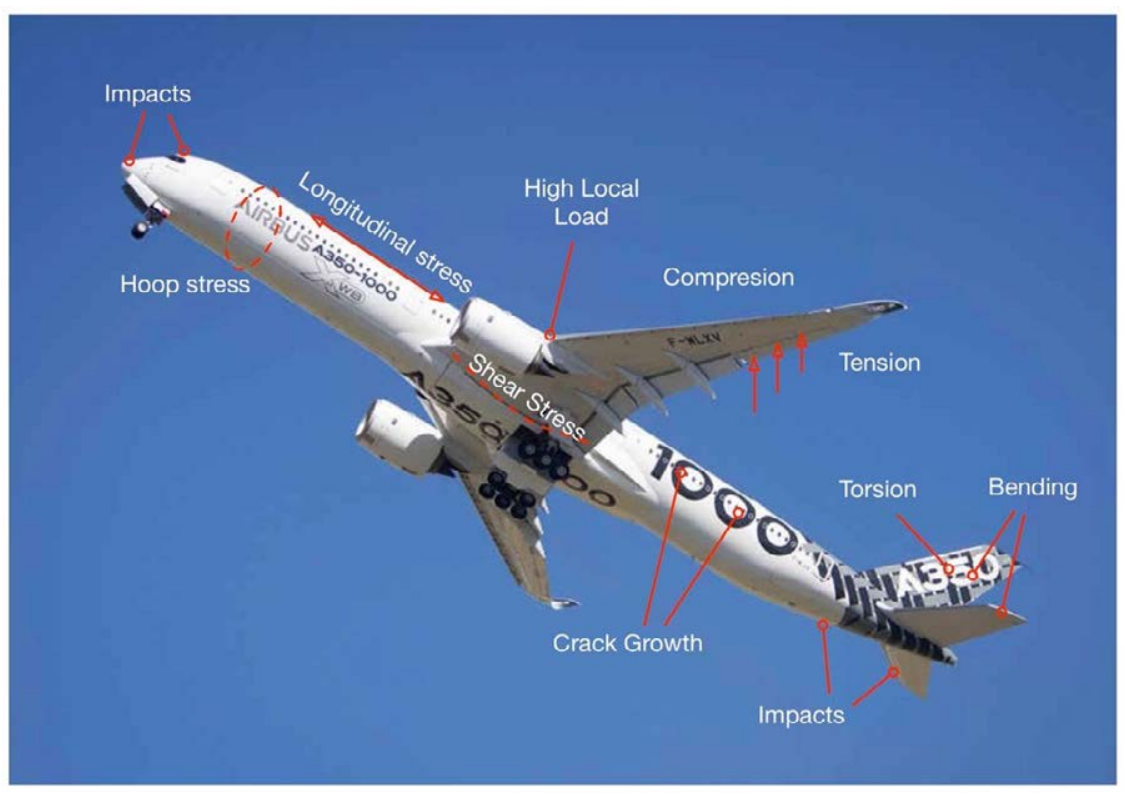

Figure 4. Summary of main loads in A/C operation. 
In general, the mentioned damages encountered in an aircraft structure can be classified as follows (Alonso García, 2016):

- Accidental damage. The source of this damage is random, and it cannot be predicted, e.g. a bird impact.

- Fatigue damage. When the structure operates under load cyclic loading, a damage can be originated such as cracks.

- Environmental damage. This is also unpredictable damage caused by the operation environment of the aircraft. Corrosion and moisture can damage the structure severely.

- Operational damage. This damage cannot be predicted, and it occurs under abnormal aircraft operations, e.g. hard landing, turbulence or overloading.

Normally, the source of the damage falls into two or more of these categories.

\subsection{Thesis Outline}

This thesis is focused on the local and global approaches to detect and locate damage in representative aeronautical structures through distributed strain measurements. Therefore, different methodologies and algorithms to process the data have been developed and they will be presented throughout the next chapters. Four main parts make up the structure of this work:

- The first part of this thesis is mainly theoretical. This section aims to describe the current state-of-the-art distributed fibre optic sensors. Thereby, a historical overview of the fibre optic sensing techniques is described with the presentation of different applications. Finally, a specific chapter has been dedicated to the measurement theory of optical backscatter reflectometer which has been employed to acquire the distributed fibre optic measurements here presented.

- A section in particular is destined to describe the damage detection methodology applied to the experimental validation results, which is presented in the second part of this thesis.

- The third part analyses the local damage detection by means of distributed strain measurement. Most of these experimental tests have been carry carried out in the Centre of Composite Materials and Smart Structures. The aim of this section is to develop and test the basis to identify different damages through distributed strain measurement.

- The fourth part of this thesis address the global damage detection in stiffened composite panels which are representative of a regional aircraft cabin. After the analysis of damage detection, the conclusion and results are presented to evaluate the capabilities for structural health monitoring of this technique. 


\subsection{Thesis Objectives}

The main objectives can be summarized as follows:

- Describe and analyse the capabilities of the different distributed measurement techniques as part of a structural health monitoring system through experimental tests.

- Demonstrate the influence of the signal conditioning parameters for distributed strain measurements. More specifically, the combination of the gauge length selection and the sensor spacing to perform a reliable distributed strain measurement regarding the expected full-field strain under external loads.

- Detect different types of local damages in composite structures through distributed strain measurements, such as delamination damage and debonding monitoring. This objective also includes the development of different algorithms and methodologies to pre-process the distributed strain measurement.

- Structural health monitoring of a real aircraft structure through distributed strain measurements in comparison with conventional techniques such as NDT for nondestructive inspection, back-to-back strain gauge rosettes for single strain measurement and high-speed camera.

\subsection{Available Resources}

This thesis has been mainly carried out at the Centre of Composite Materials and Smart Structures (CCMSS) located at the Polytechnic University of Madrid (UPM) ${ }^{1}$. All systems and equipment that have been used in this thesis has been available at the CCMSS department. They can be summarized as follows:

- Carbon Fibre Reinforced Polymer (CFRP) prepreg plies to manufacture composite coupons.

- Aluminium plates to manufacture coupons.

- Representative aeronautical structures for laboratory experiments.

- Optical Backscatter Reflectometer (OBR) 4600 for distributed measurement acquisition and data pre-processing.

- OBR switch to enable multiple distributed measurements acquisition.

- Fibre optic sensors:

\footnotetext{
${ }^{1}$ https://www.aero.upm.es/departamentos/smart-composites/index.html
} 
- Single Mode Fibre (SMF) $-28 \mathrm{e}+^{2}$ with acrylate coating.

- Fibre optic with polyimide coating.

- Fibre Bragg Gratings (FBGs).

- Fibre Connector / Angle Physical Contact (FC/APC) connectors.

- Fusion splicer Fujikura.

- Signal processing developed in software environments:

- The Mathworks, MATLAB.

- Analytical finite element model software:

- Patran.

- Nastran.

${ }^{2}$ Corning $®$ SMF-28e+ (http://www.corning.com/WorkArea/showcontent.aspx ?id=41261) 


\section{Chapter 2 FIBRE OPTIC SENSORS}

This chapter provides the fibre optic sensors that have been state-of-the-art since their inception. Thereby, a review of the introduction of the fibre optic sensors from the beginning of telecommunication applications to the most skilled sensing techniques is analysed. The three key distributed optical sensing techniques are presented and compared. Furthermore, some experimental tests performed throughout the realization of this thesis are also shown in order to evaluate the capabilities of the different fibre optic techniques. Finally, the aim at the end of this chapter is to ease the reader into comprehending of the different ways to obtain a distributed measurement by fibre optics.

\subsection{Fibre Optic Sensors Overview}

The distributed fibre optic sensing (DFOS) technique was possible thanks to the laser invention in 1960. Then, DFOS grew as a particular field of opto-electronics (Rogers, 1999). This invention offered the opportunity of transmitting light at very high frequency $(\simeq$ $10^{14} \mathrm{~Hz}$ ) which increased the interest in information transfer. Due to the realization of the light not being able to be transmitted freely through the atmosphere the attention was focused on guided-waves to protect the line paths during transmission. Furthermore, during the mid-1960s the most important breakthrough established the optical fibre category with the invention of the first low-loss optical fibre and its manufacturing process. A total attenuation of $20 \mathrm{~dB} / \mathrm{km}$ was achieved in a single-mode fibre with a diameter of 100 micron. Without this invention, the development of the telecommunications industry would not have been possible (Maurer \& Schultz, 1972).

Guided-wave optical sensors were developed at the end of 1960s as it was stated in Optical Fiber Sensors (Chester, Martellucci, \& Scheggi, 1967). Furthermore, in the same year, the Fotonic Sensor was patented ${ }^{3}$ which consisted of a bundle of fibres used to illuminate a surface and receive the reflected light from it (Menadier, Kissinger, \& Adkins, 1967). After a correct calibration, the distance between the end of the fibre and the surface can be obtained. But it was not until the 1970s when fibre optics started to be commonly used for several

${ }^{3}$ U.S. 03327584 granted June 27, 1967 
applications related to data transmission. This period also coincided with the growth of the telecommunications. Another demonstration of displacement sensors by means of two largediameter optical fibre was developed by Powell (1974). This sensor was based on the basic idea of the angle that formed one of the optical fibres, which was acting as a transmitter, to one another which was considered a receiver. At the end of this decade, fibre optics started to be considered a potential sensor able to replace the conventional electrical strain gauges. Hence, Butter \& Hocker (1978) demonstrated the ability to measure strains in a cantilever beam by means of the motion of fringes in an optical interference pattern. Furthermore, first interferometers built through single mode fibre started to appear. One year later, an interferometer was successfully developed to measure temperature and pressure changes through the same methodology of optical fringes observation (Hocker, 1979). The developed interferometer is presented in Figure 5, where the simplicity of the optical systems which provide a measurement should be pointed out.

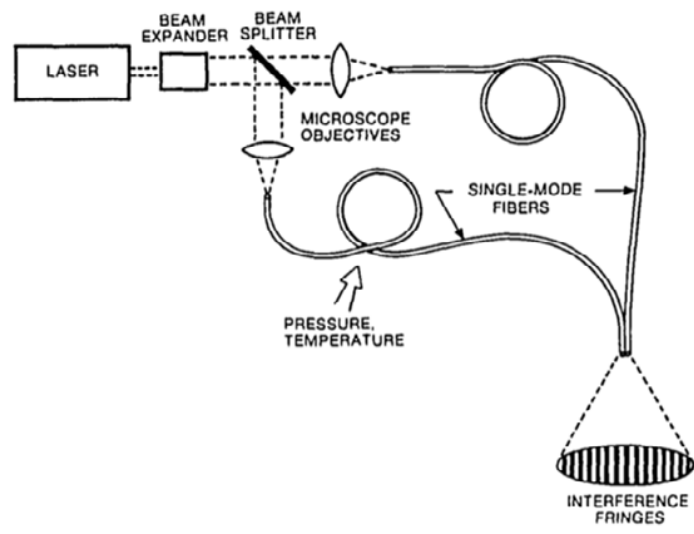

Figure 5. Single-mode interferometer for pressure and temperature measurement based on interferent fringes observation (Hocker, 1979).

The first DFOS system developed to measure the fibre attenuation characteristics was the optical time-domain reflectometry (OTDR), whose methodology was introduced by Barnoski \& Jensen (1976). Through this work, Rayleigh backscattering was recorded as the light pulse propagated down the optical fiber core. Thus, the attenuation of the optical fiber all along the fiber could be recorded and spatially distributed. Later, other sensitive parameters could be measured such as pressure, temperature and strain. After this system was invented in 1977, (Barnoski, Rourke, Jensen, \& Melville, 1977) numerous applications were used in optical fiber communication domain.

At the beginning of 80s the optical frequency domain reflectometry (OFDR) was developed in order to mitigate the difficulties encountered in OTDR in launching short powerful pulses into single-mode fibers (Eickhoff \& Ulrich, 1981). This finding achieved spatial resolutions in the order of $0.5 \mathrm{~m}$ when the frequency swept was lower enough.

Afterwards, the next DFOS technique, which will be deeply described in next chapters, was also discovered: the acoustic sensitivity of Brillouin effect in single mode fibres (Lagakos, 
Bucaro, \& Hughes, 1980). This finding allowed to build fibre optic acoustic sensors with a 5\% of error for low numerical aperture. Finally, the first experimental distributed measurement technique by Raman technique was demonstrated by measuring the temperature profile over dozens of metres fibre length (J.P. Dakin, Pratt, Bibby, \& Ross, 1985). At the time, the resolution of the distributed Raman measurements was better than $10^{\circ} \mathrm{C}$ and $3 \mathrm{~m}$. Throughout the next years, fibre optic sensors technology quickly grew in a variety of fields.

Later, in the early 90s, the Brillouin scattering was also exploited to obtain distributed temperature measurements. Thereby, in the experimental test carried out by Kurashima, Horiguchi, \& Tateda (1990) a temperature distribution was obtained in a SMF with an accuracy of $3^{\circ} \mathrm{C}$ and $100 \mathrm{~m}$ over $1.2 \mathrm{~km}$ of fibre length. Despite, the temperature accuracy was enhanced the spatial resolution was still a major challenge to deal with. Furthermore, at this time the first Michelson interferometer independent of the fibre optic polarization state was described (Kersey, Marrone, \& Davis, 1991).

Another important development occurred in the early years of the 90s with the finding of FBG engraving through phase mask technique (Meltz, Morey, \& Glenn, 1989) (K. O. Hill, Malo, Bilodeau, Johnson, \& Albert, 1993). Although, the formation of the permanent gratings had already been demonstrated (Kawasaki, Hill, Johnson, \& Fujii, 1978). Since this progress, the field of optoelectronic experienced a strong development (Kersey \& Morey, 1993) and many researchers guided their efforts towards the enlargement of the capabilities of these devices. Thus, significant progresses were achieved during this period in FBG technology and the reader can get reference from the literature (Kenneth O. Hill \& Meltz, 1997)(Kashyap, 2010).

Regarding the DFOS techniques, towards the end of 90s both strain and temperature measurements were achieved by using a novel technique based on Brillouin optical fibre frequency-domain analysis (BOFDA) (Garcus, Gogolla, Krebber, \& Schliep, 1997). Here, in this work, the spatial resolution was enhanced up to $3 \mathrm{~m}$ compared to those obtained by Kurashima et al. in the previous work.

Furthermore, during this time the OFDR technique involved an important growth since several experimental and theoretical developments were carried out to test the components and assemblies in the optical fibre networks (Passy, Gisin, von der Weid, \& Gilgen, 1994; von der Weid, Passy, Mussi, \& Gisin, 1997). These developments were driven by the achievement of narrow bandwidth of the tuneable laser sources, which enables to obtain spatial resolutions in the order of millimetre for dozens of kilometres. Thus, Froggatt \& Moore (1998) set the basis of what would later be the distributed measurement technique of the Optical Backscatter Reflectometer. In this work, an accuracy of $10 \mu \varepsilon$ over $30 \mathrm{~cm}$ interval of fibre length with $6 \mathrm{~mm}$ of spatial resolution was obtained. The methodology of crosscorrelation the Rayleigh scatter spectra for both strain and unstrained measurement was first successfully demonstrated in this experimental test to obtain the applied strain. After this work, a novel technique for distributed fibre-optic sensing based on Rayleigh backscattering involved significant progress towards DFOS techniques (Gifford, 2005; Soller, Gifford, Wolfe, \& Froggatt, 2005; Soller, Wolfe, \& Froggatt, 2005). 
Nowadays, the sensing parameters measurable by OFDR technique has been extended, not only for distributed strain and temperature measurement but also for stress vibration, 3D shape, magnetic field and so on (Ding et al., 2018). And, in general terms, the FOS field is still ongoing in several application fields (Bao \& Chen, 2012; Leung et al., 2015).

Afterwards, the growth of the FOS field over the next decades has been driven for significant cost-reduction in fibre optics, as well as, the development of a wide variety of fibre optic sensing technologies and applications (Di Sante, 2015). For some time now, the cost of the fibre optics has decreased and, despite the demand having increased, the interrogation systems are still too expensive compared to its competitors. In response to the growing interest and the potential of this technology, more solutions at a lower cost are being investigated (Liu et al., 2013).

A timeline of the most relevant findings in FOS technology previously described is shown in Figure 6.

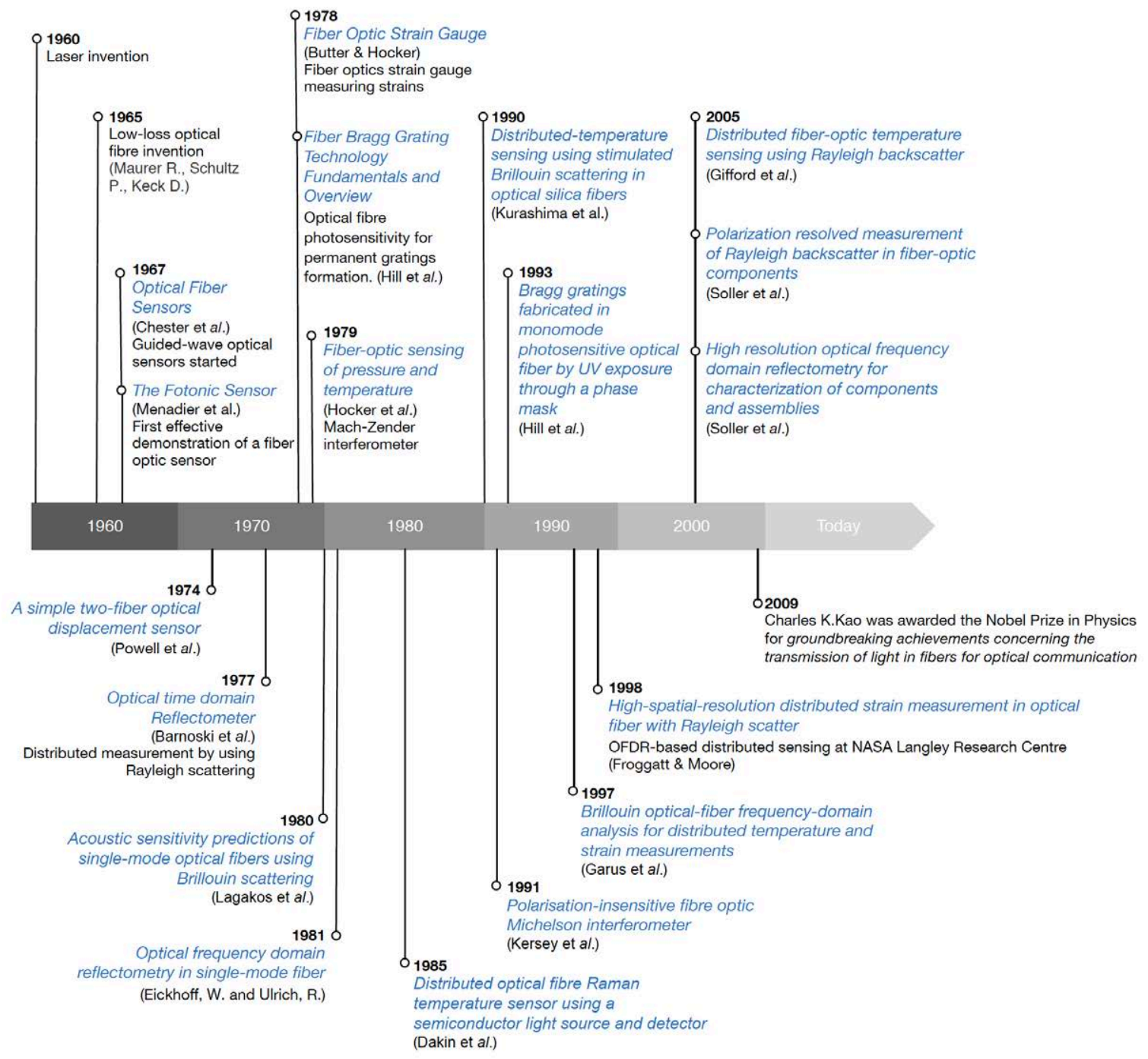

Figure 6. Timeline of main findings and progress in fibre optic sensors. 


\subsection{Fundamentals of Fibre Optics}

In this section, general concepts of the fibre optics sensors will be described in order to provide an overall understanding of this technology. Starting from the principal optical fibre parts and main classifications of the FOS to the advantages of the usage of these sensors at present.

\subsubsection{Fibre Optic Structure}

As a general definition: an optical fiber is a glass manufactured in fused silica whose principal use is to transmit light over large distances with very small losses. Light is kept in the core of the optical fibre due to refractive index of the optical core $\left(n_{1}\right)$ which is far greater (1.46 for silica) than the cladding one $\left(n_{2}\right)$. While light travels down the length of the optical fibre core, incident rays continuously hit with the cladding. As long as the angle of the incident ray is greater than the critical angle $\theta_{C}$, they will experience several internal reflections. The critical angle can be expressed in Equation 2-1. When the fibre optic exceeds a certain bending angle, or it is abnormally twisted, the transmitted light can escape.

$$
\theta_{C}=\sin ^{-1} \frac{n_{2}}{n_{1}}
$$

In the desirable case, a total internal reflection is achieved all along the length of the optical fibre with very small losses. Furthermore, the relationship between the refractive index of the fibre core and the cladding is called numerical aperture (NA) and it can be expressed through the Snell's law in Equation 2-2.

$$
N A=\sqrt{n_{1}^{2}-n_{2}^{2}}
$$

The main parts of an optical fibre are shown in Figure 7. Not only is it made up of cladding and core, but also a coating is a fundamental part of any optical fibre. As explained above, the cladding and the core are essential to achieve a total internal reflection of the light, whereas the coating handles with the protection of the optical fibre against harsh environments. There are several types of coating, which should be selected regarding the operating environment of the sensor. Most of experiments carried out in this thesis used optical fibre coated with acrylate. In some cases, with high temperature requirements, a polyimide coating was selected since it has a thermal resistance up to $400{ }^{\circ} \mathrm{C}$. Other coatings can also be made with metal, such as aluminum or gold. Furthermore, an additional outer jacket is commonly used in telecommunications to cover the optical fibers to avoid cracks made of plastic and reinforced with Kevlar.

Another fundamental characteristic of the optical fibres is the group index $n_{g}$, also called effective group index of refraction. It is defined as the ratio of the speed of light in vacuum to 
the group velocity (Todd, 2005). This parameter is a specific characteristic for each type of optical fibre.

The fibre length distance $(z)$ as a function of time, $(t)$ can be calculated as shown in Equation $2-3$, where $v_{F O}$ is the speed of light in the optical fibre.

$$
z=v_{F O} t=\frac{c}{2 n_{g}} t
$$

\subsubsection{Single-Mode Fibres and Multi-Mode Fibres}

A first classification of the optical fibre can be performed regarding the number of allowed light modes: multi-mode fibre (MMF) (Figure 7a) and single-mode fibre (SMF) (Figure 7b). As the names suggest, an MMF allows multiple light propagation modes while a SMF only allows one mode.

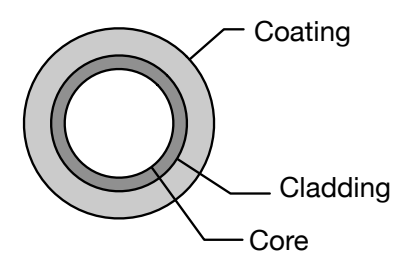

Multi-mode fibre MMF

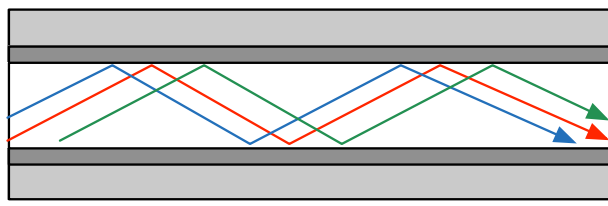

(a)

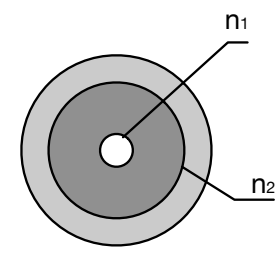

Single-mode fibre SMF

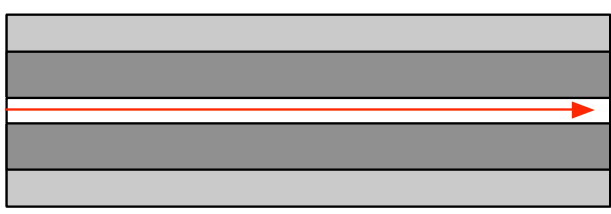

(b)

Figure 7. Main parts of an optical fiber. (a) Multi-mode fibre and (b) single-mode fibre.

Next Equation 2-4 defines $V$ as a parameter to approximate the number of modes that an optical fibre allows. It can be noticed that this parameter is clearly related to the numerical aperture and it is characteristic of each fibre. Thus, the bigger NA, the higher number of propagation modes are allowed.

$$
V=\frac{2 \pi a}{\lambda_{0}} \sqrt{n_{1}^{2}-n_{2}^{2}}
$$


where $a$ is the radius of the optical fibre core and $\lambda_{0}$ is the wavelength of the propagating light.

The Figure 8 shows the relationship between the numerical aperture and the $V$ parameter. The differently represented curves are related to the Maxwell's equations for cylindrical boundary conditions which correspond to the allowed modes. As can be seen, $V$ value must be lower than 2.405 in order to support a single mode operation, designated as HE11. For $V$ values greater than 2.405 more modes can appear depending also on the NA. It should also be pointed out that each mode is doubly degenerate into two orthogonal polarization states at the same wavelength due to polarization (Krohn, MacDougall, \& Mendez, 2014). This fact plays a significant role for the distributed measurement technique obtained by the OBR and it will be described in further detail in 3.5.1.

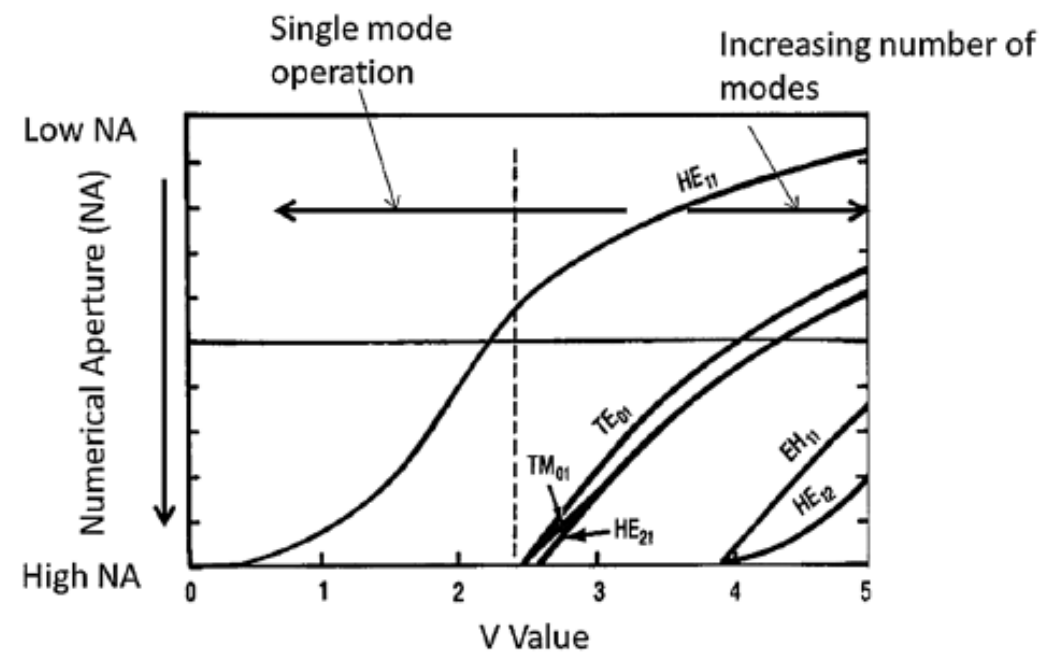

Figure 8. Mode formation with value (Krohn et al., 2014).

In general terms, it can be assumed that SMF requires small cores $(6-12 \mu \mathrm{m})$ and thus, lower NA values. Then, the major problem related to low NA values lies in the susceptibility of fibre bending since this can promote optical losses. However, regarding MMF which can support several modes of propagation, higher radius of fibre core $(50-200 \mu \mathrm{m})$ numerical aperture values are necessary.

On the other hand, Figure 9 illustrates the influence of the wavelength related to the optical signal attenuation. Therefore, the higher wavelength value used in the light propagation, the lower attenuation is experienced. As can be seen in this figure, single mode fibres are strongly dependant on this parameter and present a minimum at a $1550 \mathrm{~nm}$ of wavelength. As it will be explained in section 3.1, the centre wavelength of the SMF used by the OBR system to interrogate the fibre is also $1550 \mathrm{~nm}$ due to the lowest dispersion. Unlike SMF, the MMF are hardly influenced by the increase of the wavelength (no cut-off is appreciated). 


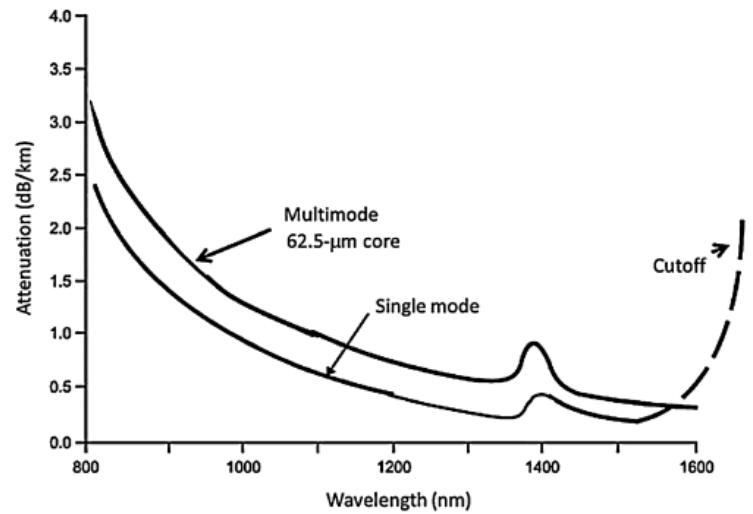

Figure 9. Attenuation in terms of wavelength (Krohn et al., 2014).

Summarizing, the SMF has lower attenuation at the same wavelength as MMF, but higher losses can be recorded due to fibre microbending and twisting. However, this type of configuration has lower dispersion than MMF and the transmitted output pulse is as sharp as the input pulse. Despite the dispersion of the MMFs for the output signal, they have an important advantage that, due to its large core diameter, the alignment of the optical fibre components and systems becomes easier. For this reason, MMFs are widely in telecommunications for transmitting light over large distances.

\subsubsection{Advantages of Fibre Optic Sensors in SHM for Aircraft Structures}

The advantages of fibre optic sensor usage are wide and varied which have turned into one of most suitable technologies for structural health monitoring, especially for aircraft structures. As is well known, weight savings is a key requirement in the design of these structures allowing to acquire high specific properties. Furthermore, they usually operate under harsh environments, such as electromagnetic interferences or moisture. Thereby, the use of FOS overcomes these issues while bringing many outstanding advantages such as:
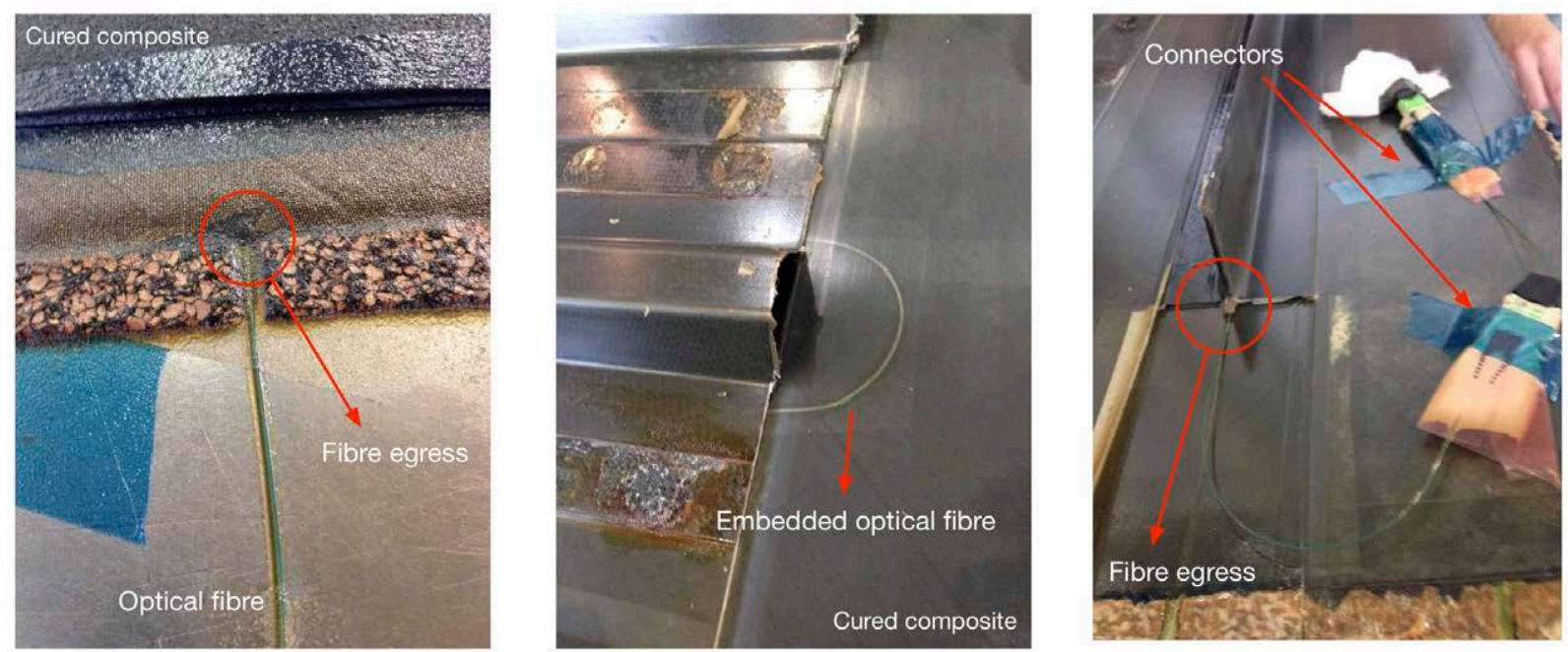

Figure 10. Example of fibre optic embedded in a stiffened composite panel. 
- Small size. The most conventional fibre optic has a diameter of 9/125 $\mu \mathrm{m}$ (core/cladding) which turns the FO into an intrusive technique for structure monitoring with no additional weight.

- Embeddable. Due to the also small size of the FO, it can be embedded in composite materials as it was demonstrated in Figure 10. In this figure, a stiffened panel with $\mathrm{T}$ and omega stiffeners were strain monitored by an embedded optical fibre with four cores (ribbon fibre type) in the last composite layer. This experimental test was successfully conducted at FIDAMC ${ }^{4}$ in collaboration with the CCMSS. Not only was the fibre able to resist the cured temperature and pressure in autoclave but also the fibre egress was successfully achieved. Several experimental projects can be found in the literature, which demonstrate the ability of embedding fibre optics in composite materials (Lesiak et al., 2012; Waite \& Sage, 1988).

- Electromagnetic field immunity. The optical fibres have no interference with the electromagnetic field which makes them one of the best choices for data communications.

- High thermal resistance. Since the core of the optical fibre is made of pure silica $\left(\mathrm{SiO}_{2}\right)$, it is able to resist very high temperatures. However, the temperature limitation is determined by the fibre coating. Then, e.g., polyimide coating can resist temperatures up to $400{ }^{\circ} \mathrm{C}$. Conventional coating of acrylate has an operating temperature range ${ }^{5}$ from $-60{ }^{\circ} \mathrm{C}$ and $85{ }^{\circ} \mathrm{C}$. From such temperature, the coating is degraded despite the core being able to remain undamaged.

- High mechanical resistance. Under a tensile proof test ${ }^{4}$, the fibre optic is able to resist $\geq 200 \mathrm{kpsi}$ or, expressed in terms of strain, $20000 \mu \varepsilon$.

- Linear response to strain and temperature. As long as the temperature or the strain field over the fibre length is increased, a linear increment in these parameters is recorded.

- Long term stability. The measurements of the fibre optic sensor have a high reproducibility since they are almost inert in harsh environments.

Figure 11 summarizes the advantages of the use of fibre optic sensors.

\footnotetext{
${ }_{5}^{4}$ http://fidamc.es

5

https://www.corning.com/media/worldwide/csm/documents/Corning\%20SMF28e\%2B®\%20Photonic \%20Specialty\%20Fiber.pdf
} 


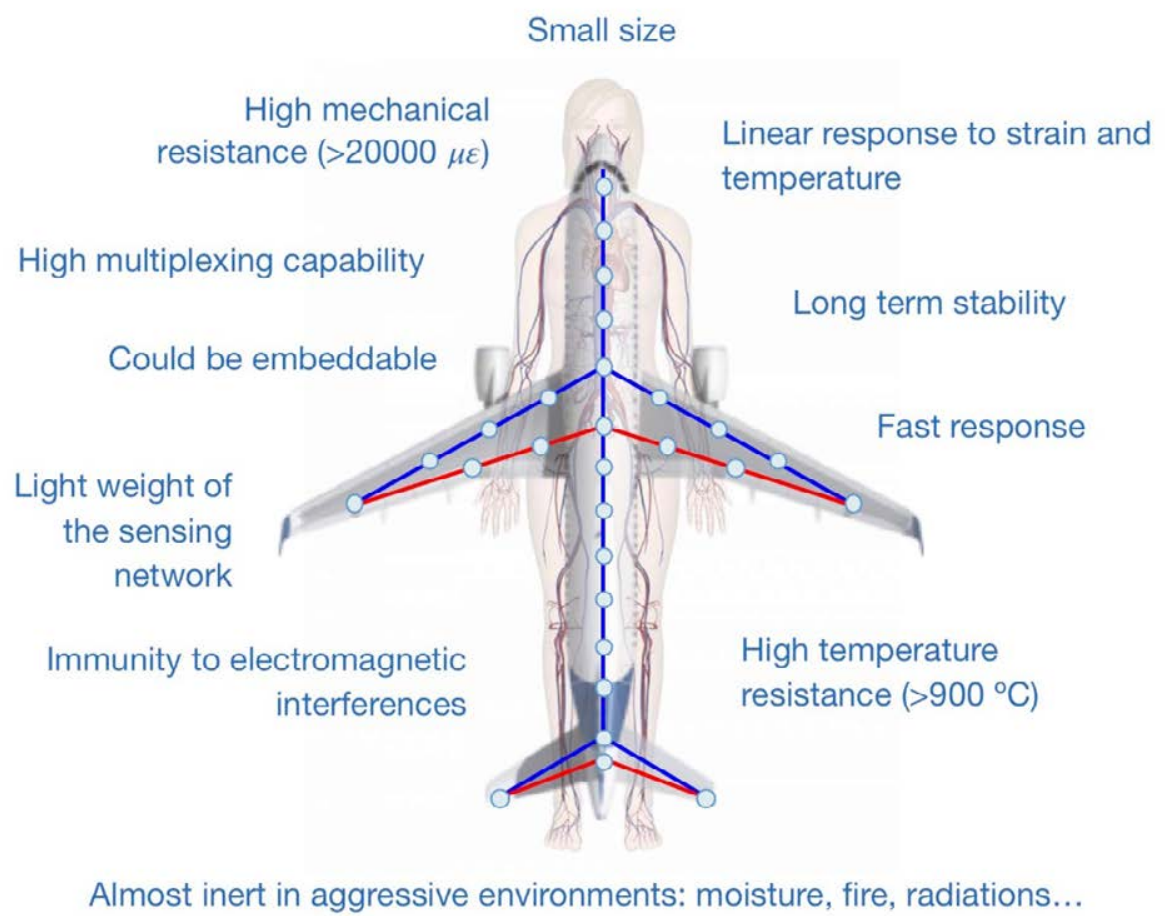

Figure 11. Main advantages of Fibre Optic Sensors.

However, it should also be pointed out that the main drawback of the optical fibre lies in its brittleness under transverse stress, the same happens in the ingress and egress of the structure. This is one of the most important matters to study in the experimental setup stage to avoid accidental breakages. Also, the location of the ingress/egress of the optical fibre deserves special attention as well as the connectors to the optical devices.

\subsubsection{Classifications of Fibre Optic Sensors}

Several classifications of the fibre optic sensors have been presented and can be found in the literature (Castrellon-Uribe, 2012; Di Sante, 2015; Grattan \& Ning, 1998).

In general terms, fibre optic sensors can be classified in three main groups:

- Interferometric sensors. These sensors enable the phase detection through a coherent light as a consequence of local length or refractive index changes by external environments. Two different measurements of light intensity are needed to detect these changes, i.e. the sum of the intensities for a reference and a perturbed measurement signal. Both signals came from the same laser source.

- Grating-based sensors. The refractive index of these type of sensors is modified to induce two main different directions of the light propagation. These fibres, socalled fibre of high level of birefringence, have an anisotropy clearly distinct from each other. 
- Distributed sensors. The main feature of the optical fibre sensors included in this group is that they are considered to be a sensor by itself, i.e. no additional action is needed to obtain a distributed measurement. Furthermore, the backscatter light, which is produced as a result of random fluctuations in the local refractive index when light is travelling through the optical core, is used to perform a distributed measurement by means of discrete sensors located all along the fibre. The three light scatterings exploited are: the Raman, Rayleigh and Brillouin scattering. These techniques will be further described in the following chapters.

This general classification and their principal sensors for each group is presented in Figure 12 .

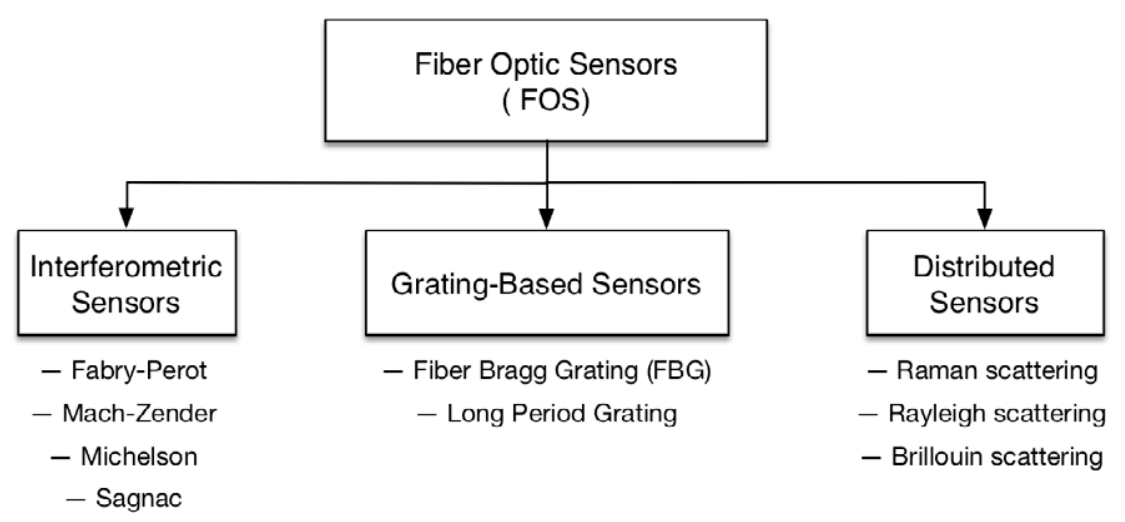

Figure 12. Classification of fibre optic sensors (Di Sante, 2015).

Moreover, another important and general classification of the fibre optic sensors is according to its topology (see Figure 13). Then, three main groups can be distinguished:

- Single point sensor. These sensors can be compared with conventional punctual sensors such as strain gauges, thermocouple or accelerometers. In this case, only one point of measurement is provided in the read-out of the sensing element. Regarding FOS field, FBGs are the most common sensor used as a single sensing element (Güemes, Fernández-López, Díaz-Maroto, Lozano, \& Sierra-Perez, 2018). At the same time, single point sensors can be classified by the measurable light property leading to four main sensor groups: intensity, frequency, phase and polarization. Further information of this classification is available in the existing literature (Menéndez, 1999; Santos \& Farahi, 2015).

- Distributed fibre optic sensor. In this case, the optical fibre is continuously sensing all along the length. A distributed measurement is built from discrete single measurement whose spatial resolution and sensing range depends on the selected technique. Then, all the optical fibre length is considered as a sensing element. The distributed sensing technique has been mentioned above and they will be meticulously described in the next sections. 
- Multi-point sensor. This is the compromise solution between a single and distributed sensor. In this group, different single point sensors are located all along the fibre length at the desired intervals in order to achieve a distributed measurement. An example of these type of sensors is described in 2.4.3.2, where several FBGs were engraved spatially distributed in an optical fibre line. In some cases, these sensors are used to obtain dynamic measurements, but in general, they present lower accuracy and spatial resolution
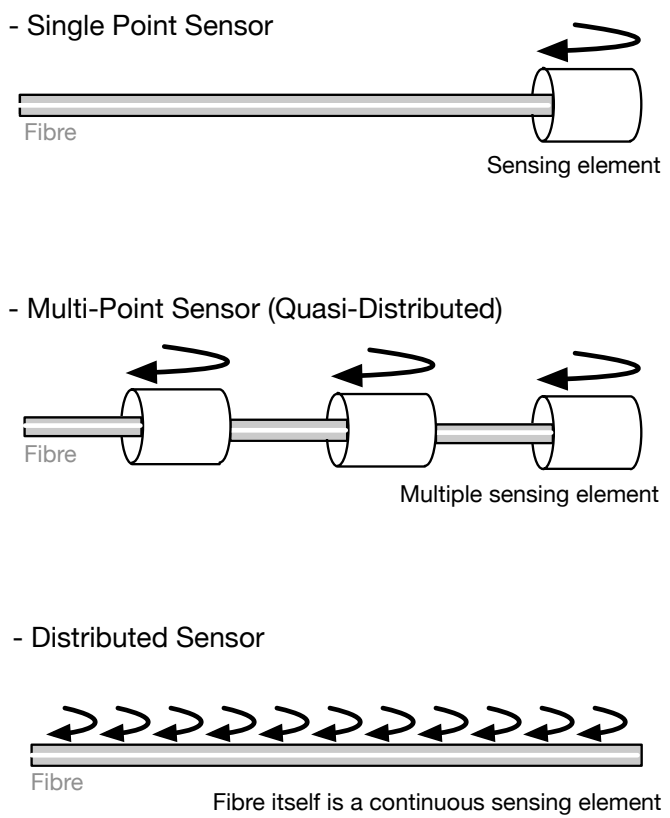

Figure 13. Classification FOS based on its topology.

In order to obtain a comprehensive view of the difference between the single point measurement and the distributed measurement Figure 14 is presented. Here, the upper measurement can be obtained by a single sensor, such as an FBG single sensor whose readout is a strain and/or temperature time history. Then the measurement obtained is represented as the dashed blue line. Instead, in the lower graph of Figure 14, an example of a distributed measurement is presented. In this case, an additional dimension is added: the fibre optic length. Hence, a distributed measurement as a function of the fibre length at a different time stamp. As long as the measurement rate increases, a strain/temperature surface can be obtained in the time domain. 

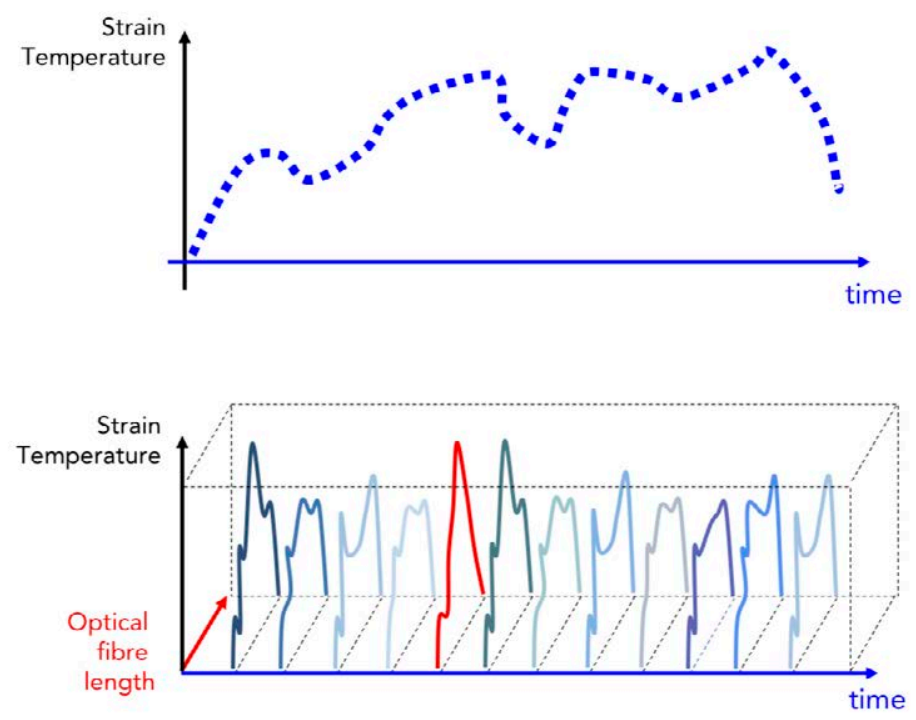

Figure 14. (Upper) Single point strain and/or temperature measurement. (Lower) Distributed strain and/or temperature measurement.

\subsection{Optical Reflectometry Techniques}

Prior to the present different sensing techniques of fibre optic sensors, optical reflectometry deserves is worthy of a description. In general terms, the optical reflectometry techniques are those based on the analysis of the reflected light waves in a specific medium, i.e., the optical system. Hence, when a light wave is propagated through a specific medium and it encounters inhomogeneities and, consequently, the light can be propagated back toward the light source. This is the so-called backscattered light which it is able to provide important and additional information about the target medium.

There are three main techniques which can be clearly distinguished in terms of reflectometry domains: Optical Time Domain Reflectometry (OTDR), Optical Low Coherence Reflectometry (OLCR) and Optical Frequency Domain Reflectometry (OFDR). These techniques have different performances regarding the measurement range, resolution, speed, sensitivity and accuracy (Soller, Gifford, et al., 2005). Whereas the OTDR is commonly used for applications with kilometers range of requirement, OLCR offers a resolution of millimeter with also a very high accuracy but in a short fiber length (roughly $5 \mathrm{~m}$ ) (W. V. Sorin \& Baney, 1992). Also the halfway solution is obtained through the OFDR technique for applications that can provide a good a combination of high speed, sensitivity and resolution all along the different length ranges (Soller, Gifford, et al., 2005).

\subsubsection{Reflectometry Characteristics}

The comparison among techniques is carried out regarding three main common features: the spatial resolution, the dynamic measurable range and system sensitivity. These features are defined in the following point: 
- Spatial resolution. This feature is referred to as a two-point resolution. In other words, it is the minimum distance necessary to distinguish two reflective events (usually of equal reflectivity). Furthermore, in some cases, the spatial resolution term is also referred to the minimum distance necessary which guarantees the strain and/or temperature resolution. For instance, the spatial resolution (twopoint) of the OBR system is $10 \mu \mathrm{m}$, i.e. minimum distance between two points of the reflective pattern, and the spatial resolution (for the distributed measurement) is $5 \mathrm{~mm}$, i.e. the separation between two sensing points (see Table 3). In practice, the spatial resolution depends on the interrogation method and the light scattering selected.

- Dynamic range. This is the total fiber length, so-called device under test (DUT), that can be interrogated by the selected optical system. This characteristic is a very important feature to take into consideration for the experimental application, i.e. the dynamic range should be in the order of the structural element dimensions.

- Sensitivity. This feature is related to the system and it is defined as the lowest backscatter power that can still be used to obtain information from the measurement.

In Figure 15 a qualitative comparison among the reflectometry techniques is presented based on the spatial resolution and the dynamic sensing range. The field of application for each technique is clearly identified regarding these performances. The OFDR field is the most varied technique since different combinations can be carried out regarding both features. In terms of sensitivity, OLCR technique obtains the highest performances with sensitivities in a range from $-140 \mathrm{~dB}$ to $-160 \mathrm{~dB}$. OFDR technique also can achieve high sensitivities around $120 \mathrm{~dB}$, while OTDR is in a range from $-60 \mathrm{~dB}$ to $-120 \mathrm{~dB}$.

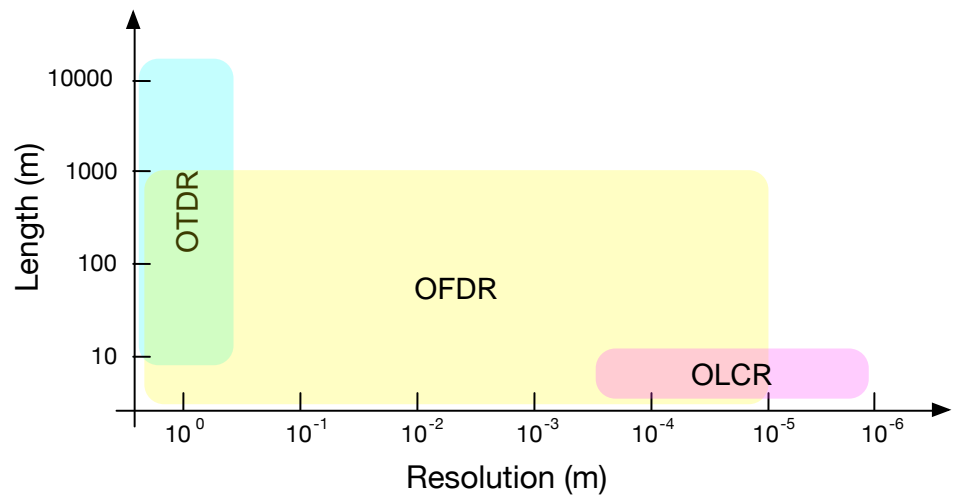

Figure 15. Comparison of reflectometry techniques regarding length and resolution of systems. 


\subsubsection{Optical Time Domain Reflectometry}

Since this technique was introduced (Barnoski \& Jensen, 1976), it has been settled as a one the most widely used for fiber characterization in telecommunication networks. The optical time domain reflectometry is used when requirements lie in long distances and the resolution of the measurement is not relevant. An OTDR measurement allows to determine the splice loss, fiber breaks location and signal attenuation with a single pulse over large distance.

A basic OTDR consists of a laser source that generates the pulse, the optical circulator or coupler to send the pulse over to the DUT and the photodetector. The normal principle of functioning is: a short light wave, i.e., the pulse, is sent down the optical fiber (DUT). Then, the photodetector receives and measures the backscatter and reflected light as a function of time by means of the relationship of the light speed and the refractive index of the optical fiber core. In most cases, this process is repeated and averaged in order to enhance the obtained results of the system network.

Figure 16 illustrates the general principle of functioning schema of an OTDR.

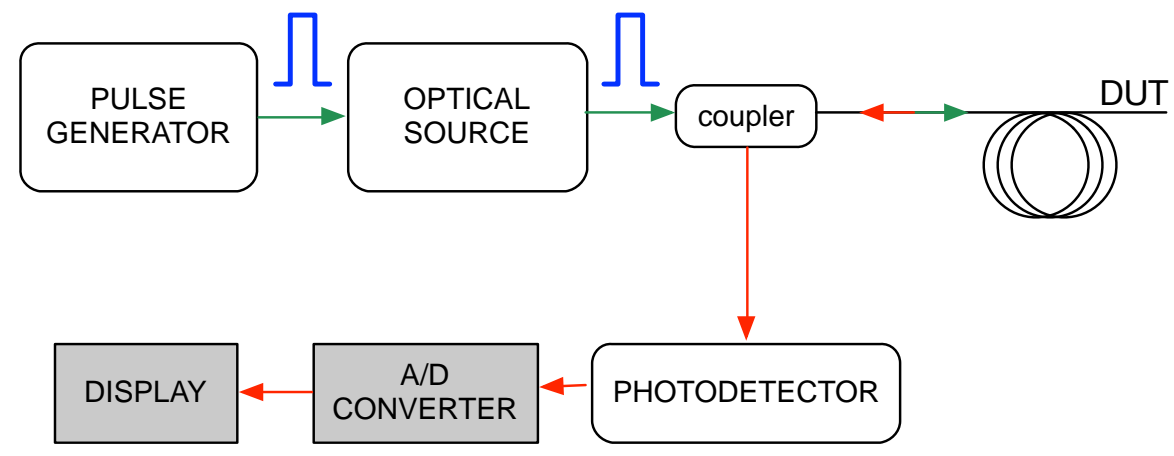

Figure 16. OTDR general principle of functioning.

The spatial resolution of the OTDR system is given by the width of the optical pulse and the response of the receiver. Therefore, the shorter length pulse, the better spatial resolution. Hence, considering the pulse width $\Delta t$, the spatial resolution $\Delta z$ is obtained as shown in Equation 2-5.

$$
\Delta z=\frac{V_{g}}{2} \Delta t
$$

Where $V_{g}$ is the group velocity of the emitted pulse (W. V Sorin, 1993). The limitation occurs when time response of the photodetector is lower than $\Delta t$. In this case, the spatial resolution of the optical system is limited by the receiver bandwidth. In SMF fibers there are some additional difficulties to send powerful short pulses and more requirements regarding the laser system, electronic and acquisition times are needed. 
Although OTDR is a powerful technique to characterize optical fiber components over large measurement ranges, the challenges regarding the spatial resolution have limited the use of this technique.

\subsubsection{Low Coherence Frequency Domain Reflectometry}

This technique is also referred to as a white light reflectometry. It emerges in 1986, achieving measurements with $10 \mu \varepsilon$ of resolution and more than $100 \mathrm{~dB}$ of dynamic range (Youngquist, Carr, \& Davies, 1987).

The basic functioning of this technique is similar to the OTDR explained in 2.3.2, however in this case the transmitted coherent light pulse into the DUT has a very short length (range of $\mu m)$. Moreover, in OLCR system, the light also travels into a so-called reference arm. The reflected light in the DUT $\left(P_{d u t}\right)$ and from the moving mirror $\left(P_{r e f}\right)$ are recombined into the fibre optic coupler and received by the detector (Pluciński et al., 2008).

A general OLCR functioning schema is shown in Figure 17.

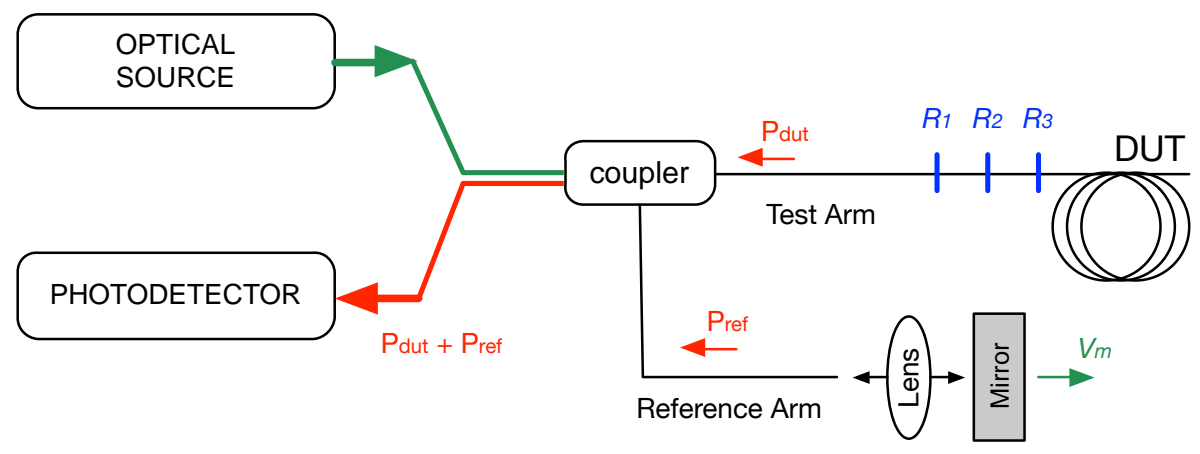

Figure 17. OLCR general schema of functioning ( adapted from Pluciński et al., 2008).

The experimental test carried out by Clivaz, Marquis-Weible, \& Salathé (1992) demonstrates the high sensitivity of this technique $(\approx-160 \mathrm{~dB})$ in a measurement range of $1 \mathrm{~m}$. Same results were reported by W. V. Sorin \& Baney (1992) with a spatial resolution of $32 \mu \mathrm{m}$ for $148 \mathrm{~dB}$ of sensitivity.

The spatial resolution of this technique can be approximated as shown in Equation 2-6 (Derickson, 1998):

$$
\Delta z \cong \frac{k}{2 n_{g}} \cdot L_{c}
$$

Where $k$ is a coefficient that depends on the light spectrum, $n_{g}$ is the optical fibre refractive index and $L_{c}$ is the coherence length of the light source. 
Despite the OLCR technique being suitable to obtain very high spatial resolution, the measurement range is too short to be considered a suitable approach for distributed sensing monitoring. Therefore, this technique is now outdated.

\subsubsection{Optical Frequency Domain Reflectometry}

The need to improve the spatial resolution of the sensing systems led to a search for solutions. Then, the first OFDR was developed in the early 80 s to obtain short powerful pulses to enable better spatial resolution approaches (Eickhoff \& Ulrich, 1981; MacDonald, 1981). This technique is also called swept wavelength interferometry (SWI), frequencymodulated continuous wave (FMCW) or coherent optical frequency domain reflectometry.

The principle of measurement for OFDR system is based on the division of a light narrowlinewidth into two interferometer arms: the reference and the DUT. Both backscatter lights of the different arms are coherently combined by the photodetectors. The reflectivity pattern is built from the detected interference fringes in the frequency domain. Through the Fourier transform the measurement is translated into the time domain, which is equivalent to obtain an OTDR measurement. The general schema is similar to that of the description represented in Figure 17.

Further detail of an OFDR system is given in 3.2 since the Optical Backscatter Reflectometer, which has been used in this thesis to obtain distributed measurements, belongs to this type of optical reflectometry.

\subsection{Fibre Bragg Gratings Sensors}

As explained in the FOS introduction (2.1), during the first years of the 90s, Fibre Bragg Gratings (FBG) (also so-called wavelength based sensors) turned into the most successful optical fibre sensor thus far (Kersey et al., 1997; Meltz et al., 1989; Rao, 1997).

Up to now, several applications have been carried out by means of FBG sensors for strain and temperature measurement in different engineering fields. It should be highlighted that the civil field to monitor bridges and wind turbine, the aeronautic field in order to evaluate the structural life of aircraft structures and even the naval field to monitor the yacht structure.

In the next sections the manufacturing of the FBG will be described, as well as the main applications of these sensors for structural health monitoring and, finally, an experimental pressurization test carried out through this technique.

\subsubsection{FBG Manufacturing}

The first observation of photosensitivity in the core of fibre optics was reported in 1978 (K. O. Hill, Fujii, Johnson, \& Kawasaki, 1978; Kawasaki et al., 1978). Then, the formation of these gratings was demonstrated by using the photosensitivity of the fibre core. 
Later, in the early 90s, the first FBG was engraved by exposing the optical fibre core to an ultraviolet laser light which created an interference pattern (Meltz et al., 1989). This is the socalled holographic technique. Figure 18 described FBG formation process. Here, two coherent UV beams from the laser source interfere at the optical fibre core to induce an index modulation of $\Lambda$ period. Also, in this figure it can be observed how the input spectrum can be acquired in either transmission $\left(\mathrm{I}_{\mathrm{t}}\right)$ or reflection $\left(\mathrm{I}_{\mathrm{r}}\right)$. Both signals show strain and temperature sensibility and then, the Bragg wavelength as a consequence of any external perturbation.

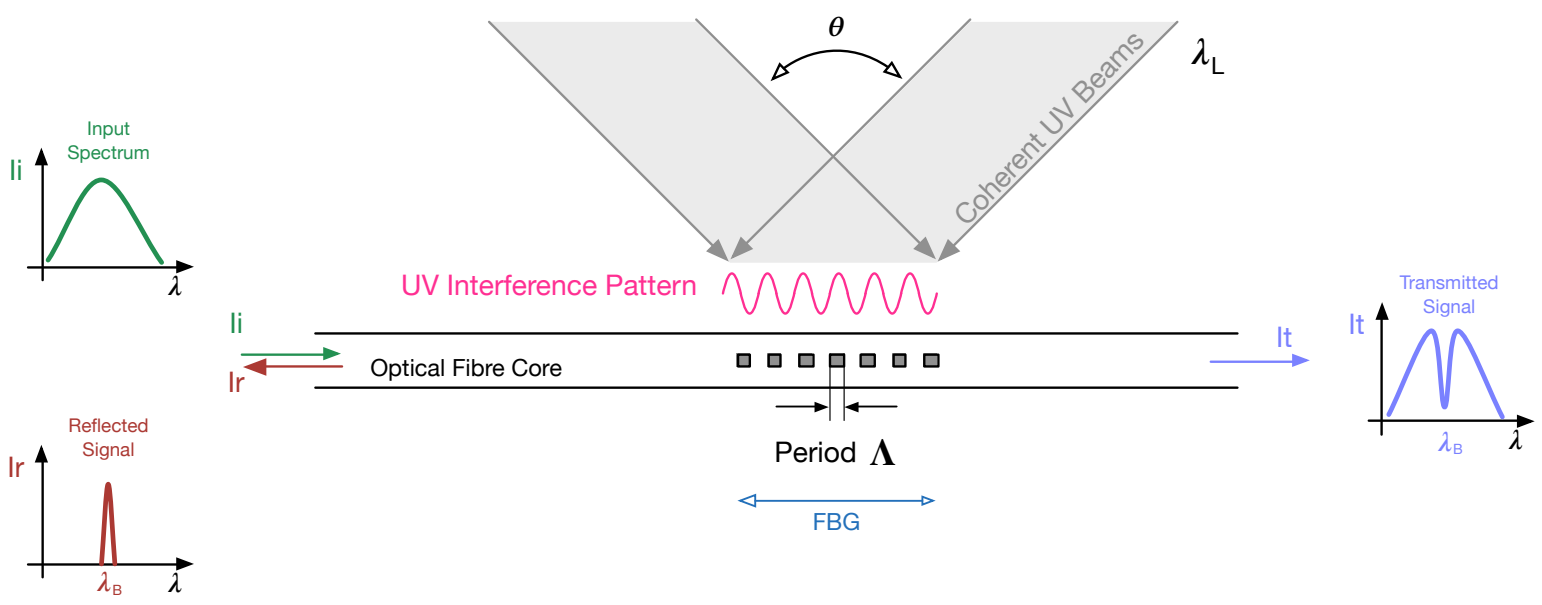

Figure 18. FBG engraved by holographic technique.

However, the most important progress of this technique was accomplished with the development of non-holographic technique to engrave FBG in 1990 (K.O. Hill et al., 1990). This novel technique was able to engrave internal grating which were also known as "Hill" gratings (K. O. Hill et al., 1978). Non-holographic techniques expose the fibre to pulsed sources or it can be masked through a spatially periodic amplitude (see Figure 19). Then, a permanent periodical perturbation in the refractive index of the optical fibre core is achieved through this process. Therefore, numerous FBGs in the same fibre can be engraved at different locations, which results in simultaneous measurement points. In the case of reflection, each of the recorded peaks by the sensing system corresponds to the Bragg wavelength at which the FBG was engraved. 


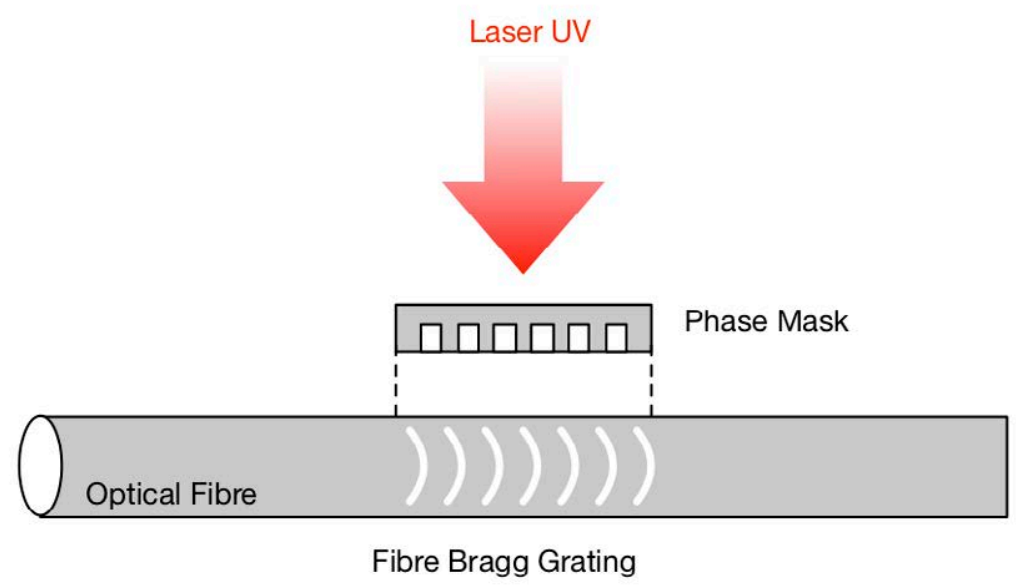

Figure 19. FBG engraved by phase mask and UV laser.

\subsubsection{Principle of Measurement}

Once the FBG is written in the optical fibre and the input light travels down all along the fibre, the reflected (or transmitted) Bragg wavelength can be calculated by the Equation 2-7. under normal incidence.

$$
\lambda_{B}=2 \overline{n_{e f f}} \Lambda
$$

Where $\Lambda$ is the modulation period, $\overline{n_{e f f}}$ is the refractive fibre optic index (averaged) and $\lambda_{B}$ is the Bragg wavelength of the grating.

Once the fibre is strained or heated, the Bragg wavelength experiences a shift proportionally to the applied load (strain and/or temperature). It should be noticed that this shift is coupled, and hence, in order to obtain the strain or the temperature separately, a compensation is needed. The changes experienced in the grating period can be approximated by the Equation 2-8 (Trutzel et al., 2000) :

$$
\frac{\Delta \lambda_{B}}{\lambda_{B_{0}}}=\frac{\Delta \Lambda\left(\varepsilon_{i}\right)}{\Lambda_{0}}+\frac{\Delta n\left(\varepsilon_{i}, \Delta T\right)}{\overline{n_{e f f}}}
$$

Where $\varepsilon_{i}$ are the six strain components (mechanical and thermal) and $\Delta T$ is the temperature change. Then through this linear relationship the measure in strain or temperature is straightforward obtained without previous calibration in the fibre lengthwise.

It should also be noted that the FBG are sensible to transverse stresses. Therefore, when the optical fibre is submitted to transverse stresses the transmitted peak at Bragg wavelength is doubled. Commercial sensors have also been developed for this purpose. 


\subsubsection{Applications of FBG}

The FBGs is considered as one the most suitable sensors to replace the conventional electrical strain gauge since they present similar features. However, FBG sensor also presents a very high multiplexing capability which is a very important aspect to consider for the monitoring of large structures. Additionally, no previous calibration is needed, and it shows a very longterm stability unlike the strain gauges.

Among the numerous application fields of the FBG sensors, aeronautical structures lead the field and they possess a large number of applications. In the following sections, two different experimental tests to monitor the strain field are presented on a composite aircraft cockpit and on a composite blade.

\subsubsection{Strain Monitoring on a Composite Aircraft Cockpit by FBG}

An example of the FBG application for strain test monitoring in real aircraft structures was carried out in a cockpit of a regional aircraft manufactured in composite material. The cockpit was monitored through a dense sensing network of FBG and strain gauges. The results here presented can be found in the conference paper entitled "Strain monitoring on a composite aircraft cabin with fiber optic sensors" (Patricia F. Díaz-Maroto, Fernández, Fernández, et al., 2016).

The FBG sensing network was divided into four channels of measurement which corresponds to the maximum number of fibre lines connected at the same time in a multiplexor. The different paths located on the cockpit surface is shown in Figure 20. The FBG sensing points are indicated with blue dots over the red lines which represent the whole length of the fibre optic paths. These lines with the specific FBGs engraved were manufactured ad-hoc in order to correctly locate the sensing points in the areas of interest. These areas were located close to the windscreen, the door, the window and the torsion box of the cockpit. This selection was based on the expected highest strain gradients over the cabin. 

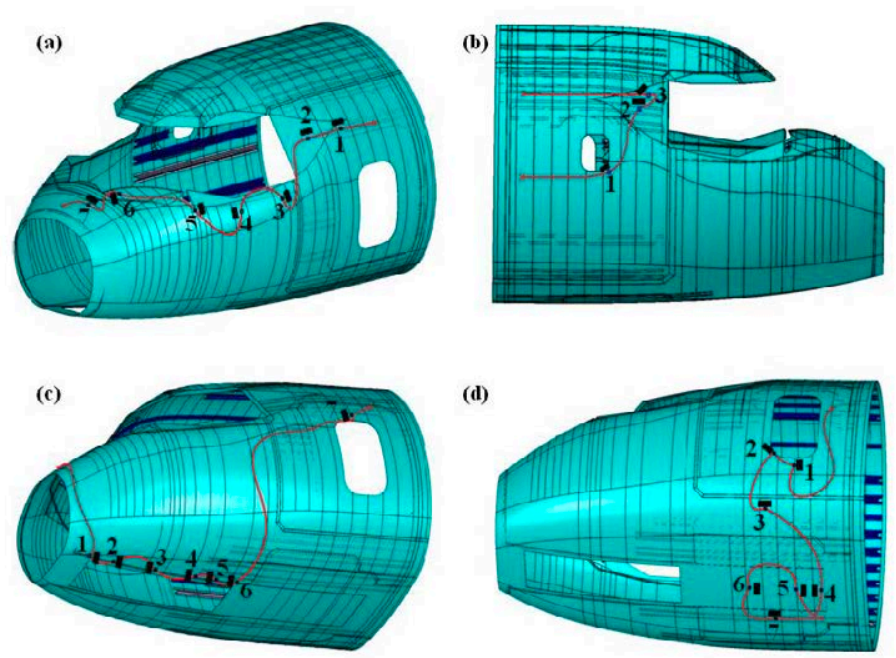

Figure 20. Four fibre optic paths which includes the FBG sensors (black squares).

The strain time-histories for each FBG sensing points are presented in Figure 21. Here, each plot corresponds to one fibre circuit. Furthermore, so as to allow the comparison, the inner pressure time-histories have also been overlapped in the same graphs. Then, the load step value can be compared with the strain distribution at each time stamp. Also, some of the FBG sensors were lost and they were not represented in the lower graphs.
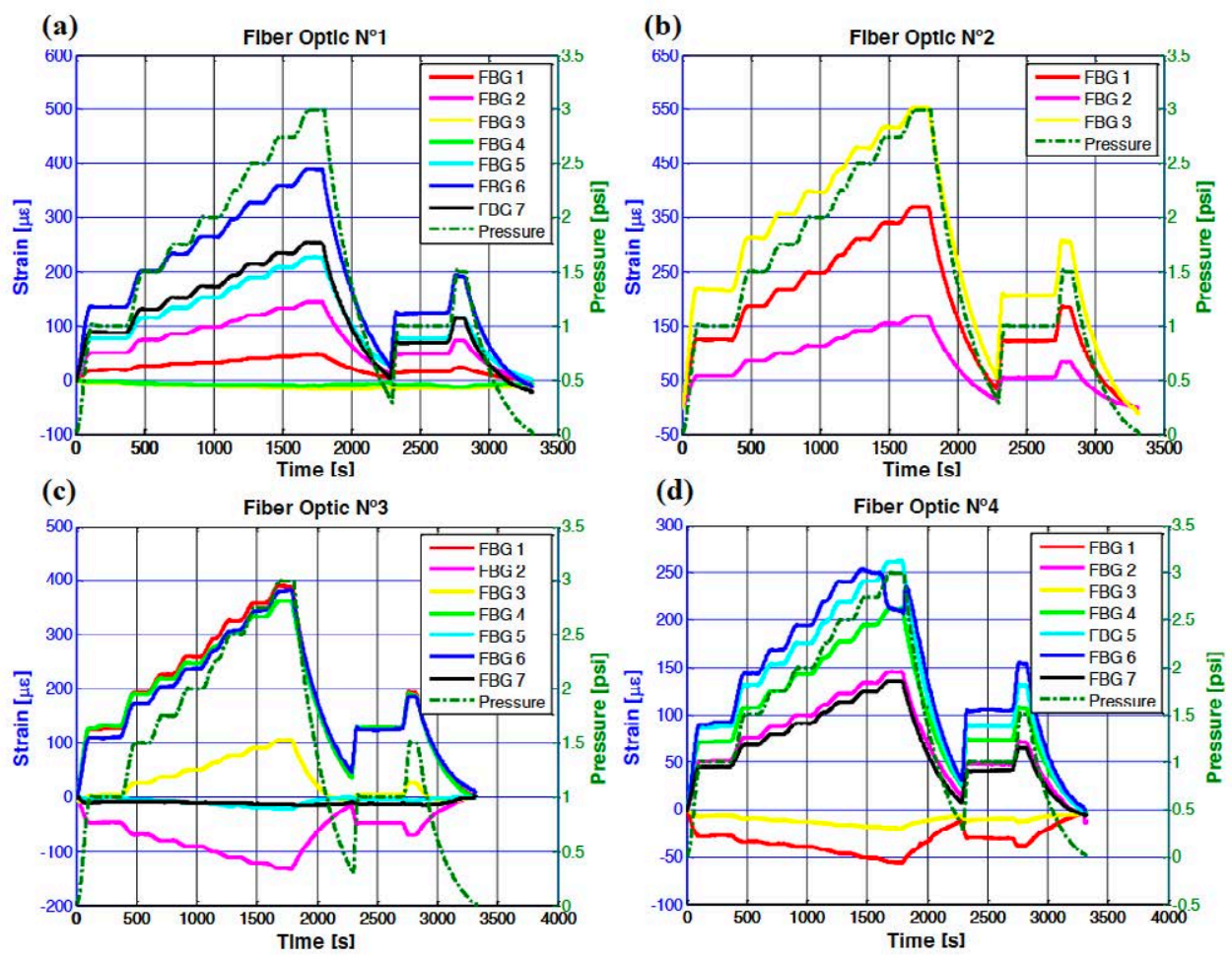

Figure 21. FBG measurements throughout the structural test. 
It should be noted that in these figures the pressure load increments can be straightforward obtained through the FBG read-out. The highest record of strain was detected in the fibre optic № 1 for the FBG №3 with $553 \mu \varepsilon$, corresponding to the closest sensor located at the cockpit ceiling. And, the minimum value was recorded in the fibre optic № 3 for the FBG № 2 with $-132 \mu \varepsilon$. This sensor was situated near the torsion box and the landing gear. Both maximum and minimum values were measured, as expected, when the inner pressure was 3 psi.

Table 1 shows the comparison between FBG measurements and strain gauges records, for an applied pressure of 3 psi.

Table 1. Comparison of the strain read-out for the FBG and strain gauge sensors at 3 psi.

\begin{tabular}{|c|c|c|c|c|c|c|c|c|c|c|c|c|}
\hline & \multicolumn{3}{|c|}{ Circuit 1 } & \multicolumn{3}{|c|}{ Circuit 2 } & \multicolumn{3}{|c|}{ Circuit 3 } & \multicolumn{3}{|c|}{ Circuit 4 } \\
\hline FBG & SG & $\begin{array}{c}\text { Relative } \\
\text { Error }\end{array}$ & FBG & SG & $\begin{array}{c}\text { Relative } \\
\text { Error }\end{array}$ & FBG & SG & $\begin{array}{c}\text { Relative } \\
\text { Error }\end{array}$ & FBG & SG & $\begin{array}{c}\text { Relative } \\
\text { Error }\end{array}$ \\
\hline No & {$[\mu \varepsilon]$} & {$[\mu \varepsilon]$} & $\%$ & {$[\mu \varepsilon]$} & {$[\mu \varepsilon]$} & $\%$ & {$[\mu \varepsilon]$} & {$[\mu \varepsilon]$} & $\%$ & {$[\mu \varepsilon]$} & {$[\mu \varepsilon]$} & $\%$ \\
\hline 1 & 47 & 23 & 104.35 & 370 & 707 & 47.67 & 389 & 378 & 2.91 & -56 & -36 & 55.56 \\
\hline 2 & 143 & 168 & 14.88 & 167 & 195 & 14.36 & -132 & -90 & 46.67 & 145 & 376 & 61.44 \\
\hline 3 & -17 & -124 & 86.29 & 553 & 401 & 37.91 & 105 & -207 & 150.72 & -20 & -29 & 31.03 \\
\hline 4 & -10 & -159 & 93.71 & - & - & - & 361 & -215 & 267.91 & 212 & 150 & 41.33 \\
\hline 5 & 227 & -26 & 973.08 & - & - & - & -21 & -35 & 40.00 & 262 & 120 & 118.33 \\
\hline 6 & 389 & 397 & 2.02 & - & - & - & 381 & 349 & 9.17 & 210 & 188 & 11.70 \\
\hline 7 & 253 & 171 & 47.95 & - & - & - & -13 & 13 & 200.00 & 136 & 169 & 19.53 \\
\hline
\end{tabular}

Significant differences between both values are detected in most of the FBG locations. These discrepancies are mostly promoted by the location of the FBG and strain gauge sensors, e.g. FBG № 4 of fibre optic circuit № 3 was located at $150 \mathrm{~mm}$ from its analogous SG. Additionally, for fibre lines № 2 and № 3, the maximum differences are registered by the couple of sensors identified by numbers 1 and 2, respectively. In both cases, the measuring devices are located in a region under the effect of high stress concentrations (window and door corners). Despite FBG sensors were positioned as close as possible to strain gauges, the presence of stress concentrations can cause large strain changes in short distances, as can be appreciated in Table 1.

Finally, great and abnormal differences were also observed in sensors № 5 (fibre optic circuit № 1). In this case, sensors were positioned on a riveted region to join a frame with the 
cockpit skin. In spite of distance between both sensors was lower than $20 \mathrm{~mm}$, large strain differences were recorded.

This experimental test shows the capabilities of the FBGs to be installed in such large structure so as to obtain discrete strain measurements at different points. The main drawback found out was the complex installation of the FBGs at the desired locations for each single fibre. Seven FBGs were engraved at different lengths in a unique optical fibre of several metres, thus the rest of the fibre (free of FBGs) was randomly allocated over the skin. This presents an important risk of accidental fibre breakage which could spoil all the FBGs engraved.

\subsubsection{Strain Monitoring on a Blade by FBG-OFDR technique}

A recent technique which combines the advantages of the OFDR and the FBG was developed to obtain quasi-distributed strain measurement by $5 \mathrm{FBG}$ with $6 \mathrm{~mm}$ of gauge length (Igawa et al., 2005). Unlike other approaches (Froggatt \& Moore, 1998a), this technique engraves FBG at the same wavelength and the DUT was interrogated by changing the tuneable laser range.

The FBG-OFDR system (Figure 22) consists of a tuneable laser source (TLS), two photodetectors, three broadband reflectors, 3-dB couplers, a long-length FBG whose length depends on the experimental test, and a computer with a high-speed analogue to digital converter. The TLS carries out a wavelength swept at a specific rate and range. Hence, the reflected spectrum at every FBG position is recorded. By means of a fast Fourier transform (FFT), the wavenumber and frequency of every reflected point can be calculated. Converting the frequency into position through the knowledge of light speed, the spectral distributed measurement is obtained. Then, the strain distribution can be also performed from that reflected spectrum obtained at each location all along the FBG.

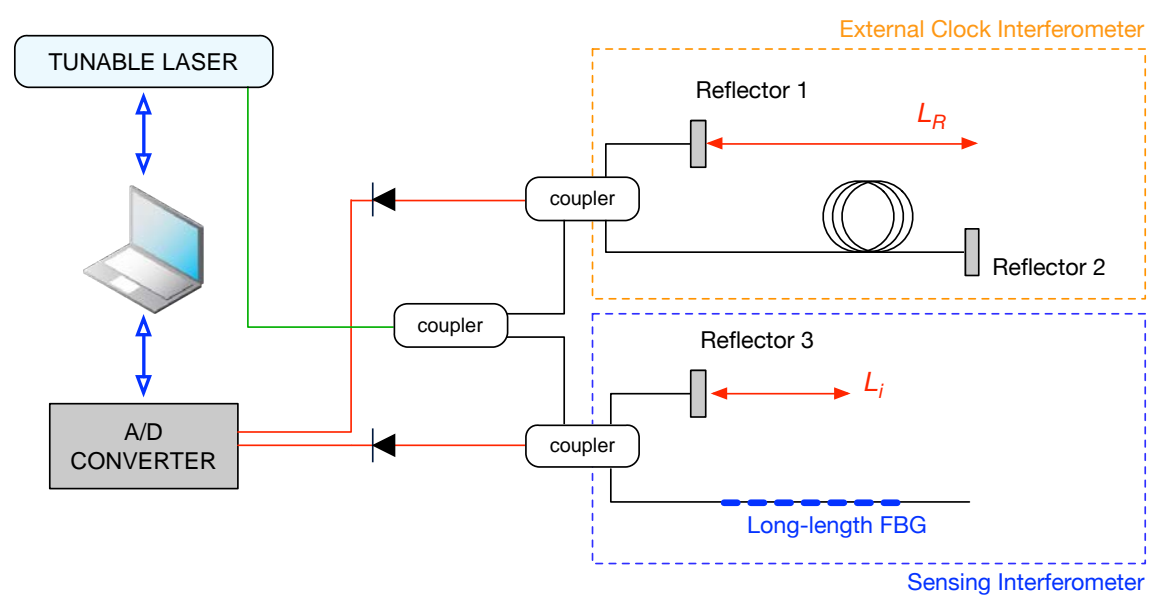

Figure 22. Schema of functioning of a FBG-OFDR system (Murayama, Wada, \& Igawa, 2013).

So as to validate the experimental OFDR measurements a finite element model (FEM) has been executed through Abaqus ${ }^{\circledR}$ software. For this purpose, two hanging loads were applied 
to the surface blade. In the first case, a load of $113 \mathrm{~N}$ was introduced close to the tip blade area. While in the second case, a load of $97.5 \mathrm{kN}$ was applied close to the middle area.

The blade finite element model has been validated through strain gauges and FBG-OFDR distributed strain measurements. In Figure 23, results from these three techniques have been presented in both cases. A suitable agreement among them has been clearly visible. Although, a small shift can be observed in case 2 due to the location accuracy of the second load in the FEM and the optical fibre positioned with respect to the blade FEM geometry.
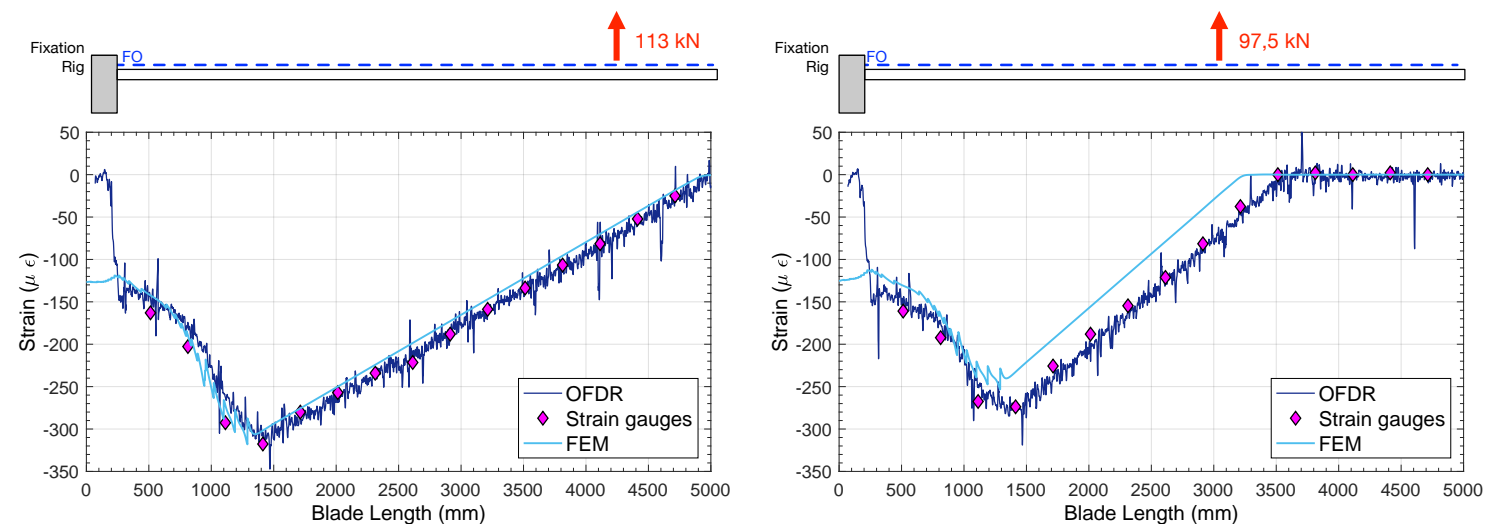

Figure 23. Experimental results for OFDR-FBG validation. (Left) First static test with a hanging load of $113 \mathrm{kN}$. (Right) Second static test with a hanging load of $97.5 \mathrm{kN}$.

\subsection{Distributed Fibre Optic Sensors}

This section focuses on three fundamental aspects to introduce the distributed fibre optic sensing techniques. First, the commence and introduction of different distributed techniques are presented. Rather recently, these techniques have become some of the most promising techniques in the field of optical sensors. Not only are they changing the way to monitor the structures, but also the data processing of the measurement acquired since large amounts of discrete sensing points are contained in a single optical fibre. Then, main features and advantages of each distributed technique as well as disadvantages are deeply described and studied. And, finally, this section concludes with a comparison among techniques based on structural monitoring requirements, such as magnitude of measurement, spatial resolution or accuracy.

Before dividing into the distributed sensing technologies, the light backscattering effect in optical fibres should be described in detail for a better understanding. The beginning of the distributed techniques dates back to the 1800s, when the optical light scattering was discovered by Lord Rayleigh based on the theory of sky colours as a result of the thermal movement of molecules. After many years, the theory of backscattering effect in waveguides was widely studied in the 1970s after the optical fibre appearance (Kapron, Maurer, \& Teter, 1972) (2.1). 
The backscattering of the light is continuously produced when it is propagated through the core of the optical fibres. In this propagation, there are photons of light that go back toward the optical source, whereas much others are traveling forward to the optical receiver (Harris \& Bertolucci, 1978). Most of these photons are elastically scattered from an atom or molecule, i.e., they retain the same energy of the incident photons, its frequency and wavelength. However, few of them tend to change this energy and scatter the light in an inelastically manner.

The three possible scenarios are illustrated in Figure 24: Rayleigh, Stokes and Anti-Stokes scattering. As is well known by the Quantum Theory of Radiation (Einstein, 1917): an electron emits a photon of frequency $v=E / h$ when it transits down to a lower energy state. Instead, an electron needs to absorb energy from photons to transit up to higher and unstable energy states, the so-called virtual states $E_{v}$. As expected, most electrons in the material occupy the ground state at a stable energy denoted by $E_{0}$. Also, some electrons occupy excited states with an energy $E_{1}=E_{0}+\Delta E$.

In a spontaneous scattering process, when an incident photon of energy $h v$ reaches the material, the electrons absorb this energy to transit up to the virtual state, which acquires energy $E_{v}=E_{0}+h v$. Because of this unstable state, the electron quickly returns into the ground state releasing a photon of the same energy (Figure 24a) or into the excited state (Figure 24b) releasing a photon of energy $h v_{S}$ (Stokes scattering). When electrons in the excited state absorb the incident photon and then, transit down to the ground state, they release an additional energy denoted by $\Delta E$. Hence the anti-Stokes scattering corresponds to an energy $E_{v}=h v+\Delta E$. These are the three interactions that may occur between photons.

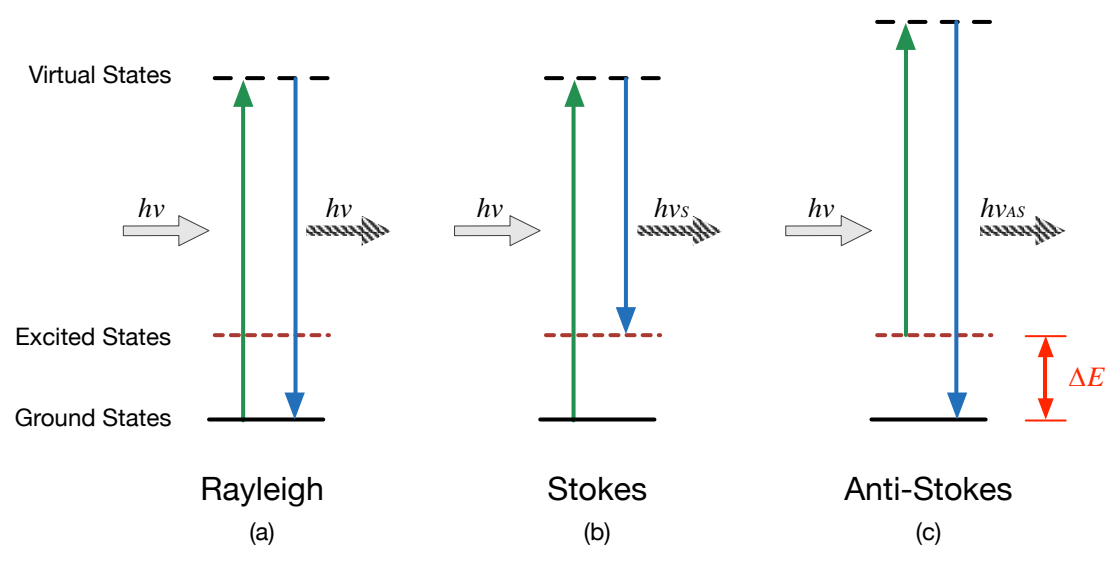

Figure 24. Stokes and Anti-Stokes scatterings (adapted from (Fang, Chin, Qu, \& Cai, 2012)).

The main inelastic light scatterings are the Brillouin and the Raman scattering. Whereas, the linear elastic scattering, as mentioned above, is the Rayleigh scattering. All of these scatterings are illustrated in Figure 25 regarding their wavelengths in which they appear. As can be seen in this figure, both Raman and Brillouin have Stokes and anti-Stokes components. However, in the case of Raman scattering, only anti-Stokes component is sensitive to temperature changes since these photons have greater energy (lower 
wavelength). The higher the temperature, the more active photons at anti-Stokes wavelength. Also, it can be confirmed that Rayleigh scattering is the strongest scattering based on the probability of elastic interaction between photons.

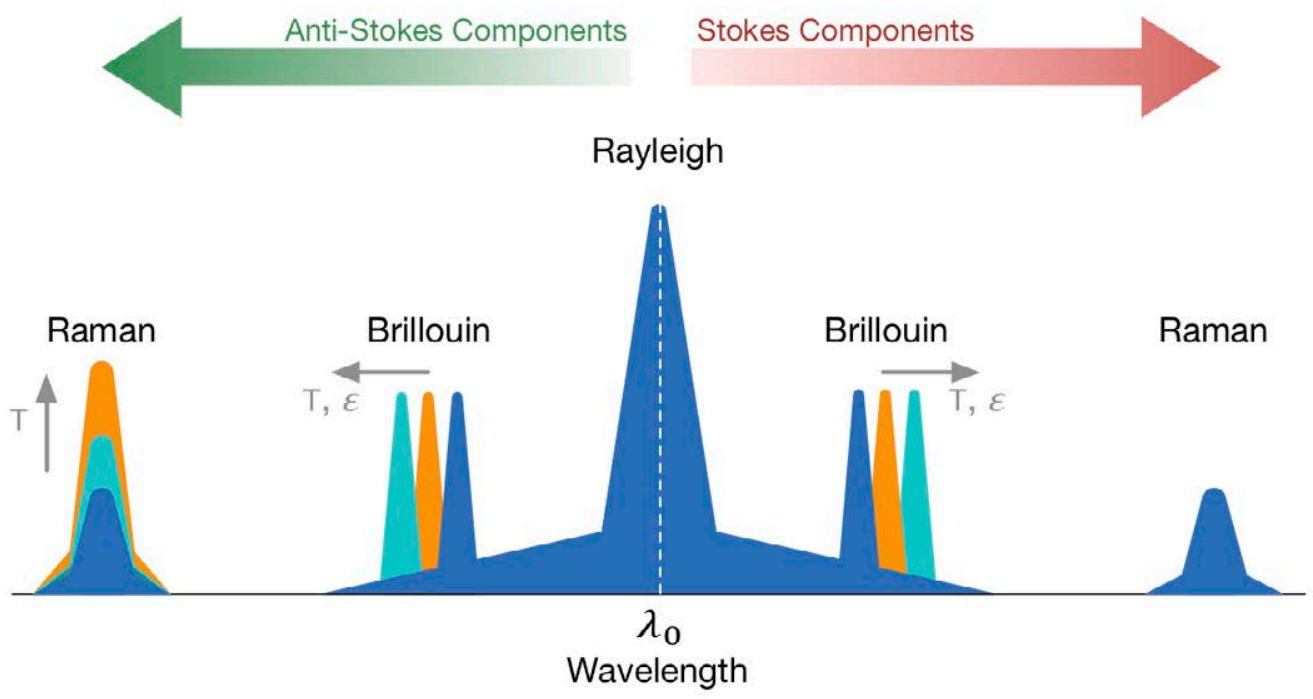

Figure 25. Light scattering: Raman, Rayleigh and Brillouin.

Moreover, in the case of non-linear scatterings, they can be stimulated so as to reinforce the acoustic waves and enhance the scattering of phonon. Then, the so-called stimulated Brillouin scattering (SBS) and stimulated Raman scattering (SRS) were obtained as a result of this technique (Tang, 1966). The stimulated scattering can be achieved at relatively high pump powers in long fibre lengths which allows to amplify the light scattering at Raman and Brillouin frequencies (Santos \& Farahi, 2015).

Later on, the three different distributed sensing techniques will be described showing some of the applications which have been successfully used.

\subsubsection{Distributed Sensors Based on Brillouin Scattering}

As explained in the previous section, the Brillouin scattering is caused by the interaction of photons and acoustic waves (phonons). First applications of Brillouin addressed the characterization of single and multimode optical fibres (Rich \& Pinnow, 1974; Tkach, Chraplyvy, \& Derosier, 1986). Later, the Brillouin backscattering was measured to build a tensile strain sensor since it was demonstrated that the Brillouin frequency shifts were sensitive to the applied strain in the optical fibres (Tsuneo Horiguchi, Kurashima, \& Tateda, 1989).

Similarly, in the case of Bragg gratings, light is reflected back by changes in the refractive index, the Brillouin frequency experiences shifts due to index modulations caused by acoustic waves. The Brillouin frequency $v_{B}$ can be calculated from Equation 2-9 (Tsuneo Horiguchi et al., 1989): 


$$
v_{B}=\frac{2 n_{e f f} V_{a}}{\lambda}
$$

Where $n_{e f f}$ is the refractive index of the optical fibre and $\lambda$ is the wavelength of the light in the glass. Then, the acoustic velocity, $V_{a}$ is obtained as shown in Equation 2-10 (Timoshenko \& Goodier, 1951):

$$
V_{a}=\sqrt{E \frac{1-\kappa}{(1+\kappa)(1-2 \kappa) \rho}}
$$

It should be pointed out that the acoustic velocity depends on Young's modulus $E$ and density $\rho$ of the optical fibre core. Thus, the BS is able to provide information about the material composition (T. Horiguchi, Shimizu, Kurashima, Tateda, \& Koyamada, 1995; Kurashima et al., 1990). Furthermore, the shift of the Brillouin frequency is linearly dependant on the temperature and strain a expressed by the Equation 2-11 (He, Zhou, \& Ou, 2014).

$$
\Delta v_{B}(T, \varepsilon)=C_{\varepsilon} \Delta \varepsilon+C_{T} \Delta T
$$

Where $\Delta v_{B}(T, \varepsilon)$ is the change of Brillouin frequency shift and $C_{\varepsilon}$ and $C_{T}$ are the strain and temperature coefficients respectively and, $\Delta \varepsilon$ and $\Delta T$, the strain and temperature changes regarding the reference measurement.

\subsubsection{Distributed Brillouin Systems}

A wide range of strain and temperature sensing systems have been settled based on the Brillouin scattering. Therefore, at the beginning of 90s, the Brillouin optical-time domain (BOTDA) analysis was tested to obtain a distributed temperature measurement with $3^{\circ} \mathrm{C}$ of temperature accuracy and a $100 \mathrm{~m}$ of spatial resolution in a range of $1.2 \mathrm{~km}$ (Kurashima et al., 1990). In general, a BOTDA system is able to obtain a distributed measurement based on the Brillouin gain spectroscopy. A simplified schema of this system is shown in Figure 26.

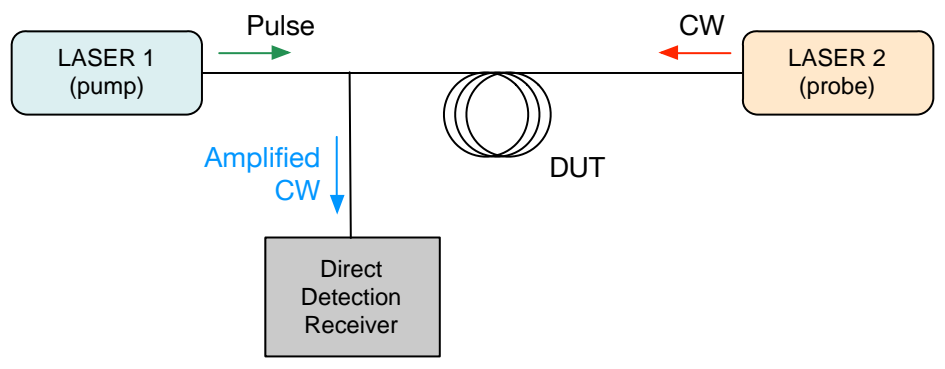

Figure 26. Schematic description of a BOTDA system. 
In this system, two waves are injected into the optical fibre: from one side (Laser 1) a pulsedoptical wave, i.e., the pump, and from the other side (Laser 2) a counterpropagating continuous wave (CW) light, i.e., the probe signal. Hence, in most BOTDA system both sides of the optical fibre are needed to measure the temperature dependence. An SBS occurs when the difference $\Delta v$ between the pump and the probe signal corresponds to the Brillouin frequency shift $\Delta v_{B}$ of the optical fibre. In this instant, the gain of the continuous wave light intensity is highly amplified which can be hundred times higher than Rayleigh scattering ( $\mathrm{T}$. Horiguchi et al., 1995). Then, the probe signal can be obtained as a function of time, such as an OTDR system.

Later, an improvement of the BOTDA system was accomplished: Brillouin optical-fibre time domain reflectometry (BOTDR) (Kurashima, Horiguchi, Izumita, Furukawa, \& Koyamada, 1993). The advantages with regarding the BOTDA are focused on only one side of the optical fibre which is used to inject the light and the sensing range that becomes therefore enhanced maintaining its accuracy and spatial resolution. A simplified schema of this system is shown in Figure 27.

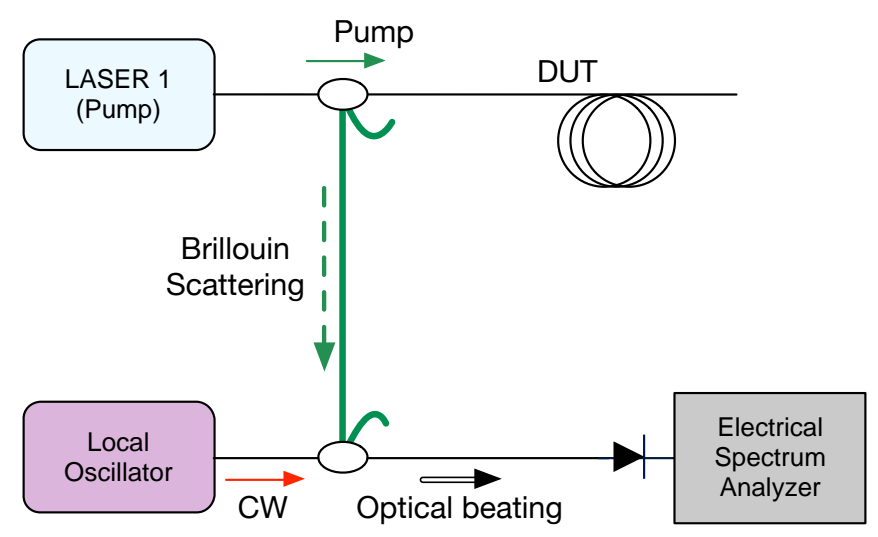

Figure 27. Schematic description of a BOTDR system.

In addition to these two first techniques, several methodologies and systems have been developed since the first BOTDA. For example, the Brillouin Optical Frequency Domain Analysis (BOFDA) which is based on the measurement of a complex baseband transfer function to relate the $\mathrm{CW}$ amplitude with the Stokes waves all along the fibre length (Garcus et al., 1997). Furthermore, a variant of BOTDA, the PPP-BOTDA which consists on using a pre-pump to measure the frequency difference between the pump and the probe waves instead of the Brillouin gain directly (Minakuchi, Okabe, \& Takeda, 2007). Also, more recently another technique Brillouin optical correlation-domain Analysis (BOCDA), in which two of the SBS are obtained by exciting it using a frequency-modulated pump and probe light waves (Hotate \& Hasegawa, 2000).

As demonstrated, several system and techniques have emerged from this technique. The simplicity of the system to obtain the Brillouin frequency has made possible the combination 
of multiple schemas to generate distributed measurements. However, the spatial resolution and the accuracy of these measurements is, in most cases, a major issue to overcome.

\subsubsection{Brillouin applications}

The applications of the Brillouin reflectometry for the SHM field is widely varied. This is due to the fact that this technique offers as much in static as dynamic distributed measurement with a considerable strain accuracy and spatial resolution. Also, the applications for health monitoring are not only limited to aerospace structures but also to civil and laboratory experiments.

One of the first applications of the Brillouin technique in an aircraft structure is found in the pressurization test under cryogenic conditions (Shimizu, Yari, Nagai, \& Takeda, 2001). Later, in 2003 the fuselage of a launch vehicle was monitored to analyse the deformations after curing (Murayama, Kageyama, Naruse, Shimada, \& Uzawa, 2003). Other small-scale experiments were also carried out, thereby bolt holes were also successfully monitored after bearing failure by using a BOCDA system (Takeda, Minakuchi, \& Nadabe, 2013).

Some experiments have attempted to enhance this characteristic in terms of measurement range, strain and temperature accuracy. Likewise, it has been demonstrated that a range of 325 kilometres can be achieved by using four repeaters (Gyger, Rochat, Chin, Niklès, \& Thévenaz, 2014). However, the spatial resolution in this case was limited to 3 metres with a temperature accuracy of $2{ }^{\circ} \mathrm{C}$. Better spatial resolution ( 2 centimetres) in 8.8 kilometres was obtained by London, Antman, Preter, Levanon, \& Zadok (2016). Similar results were obtained by Denisov, Soto, \& Thévenaz (2016). Considering these results, large measurement range can be obtained by means of wide strategies, however the spatial resolution and measurement accuracy is affected. Nevertheless, this makes it attractive to monitor large structures if the requirements in the measurement are less demanding.

\subsubsection{Distributed sensors based on Raman scattering}

Raman backscatter occurs when the light is travelling by the optical fibre length and it experiences two frequency shifts in the backscattered spectrum, Raman Stokes and Raman anti-Stokes respectively. This effect was first reported by C.V Raman (who received the Nobel Prize in 1930) and K. S. Krishnan (1928). The backscattered light received contains information about the temperature and the optical losses of the optical fibre.

The first system based on Raman technique was developed in the mid 80s (J.P. Dakin et al., 1985). An OTDR Raman-based distributed measurement system is able to measure the backscattered light of one pulse signal sent through the optical fibre and recorded as a function of the fibre length.

Therefore the temperature calculation can be elaborated by using two ratios (Bolognini, Park, Soto, Park, \& Di Pasquale, 2007), one between the light intensity of anti-Stokes component, which depends on both the temperature and optical losses, to Stokes component or between anti-Stokes intensity light to backscattered pump light. 


$$
\begin{gathered}
\frac{I_{A-S}}{I_{S}}=\left[\frac{\left(\kappa_{0}+\kappa_{k}\right)^{4}}{\left(\kappa_{0}-\kappa_{k}\right)^{4}}\right] \exp \left(-h c \frac{\kappa_{k}}{k T}\right) \\
\frac{I_{A-S}}{I_{S}} \propto\left[\exp \left(\frac{h \Delta v_{R}}{k T}\right)-1\right]^{-1}
\end{gathered}
$$

The Equation 2-12 and Equation 2-13 represent the ratio as described above, where $I_{A-S}$ is the anti-Stokes light intensity, $I_{s}$ might be the pump or Stokes light component, $k$ and $h$ are the Boltzmann and Planck constant respectively, $T$ is the absolute temperature and $\Delta v_{R}$ is the frequency separation between $I_{A-S}$ and $I_{S}$. Through this expression, the temperature can be obtained at a discrete single optical fibre point. Furthermore, since the pump injected light is a pulse of a few nano-seconds, the time of arrival can easily be correlated with the fibre position through the travel time of the backscattered light. Then, this operation can be repeated to obtain the temperature profile along the optical fibre.

A general schema of functioning of Raman-based system is shown in Figure 28. An optical pulse is coupled into the DUT by the pulsed laser. Due to the beam splitter the two intensities of Raman bands (Stokes and anti-Stokes) can be obtained separately. Then the ratio of anti-Stokes to Stokes is calculated to measure the temperature changes at any given point along the fibre. Despite the typical $0.2^{\circ} \mathrm{C}$ of temperature resolution (Glišić \& Inaudi, 2007), the spatial resolution is generally limited by the order of meters.

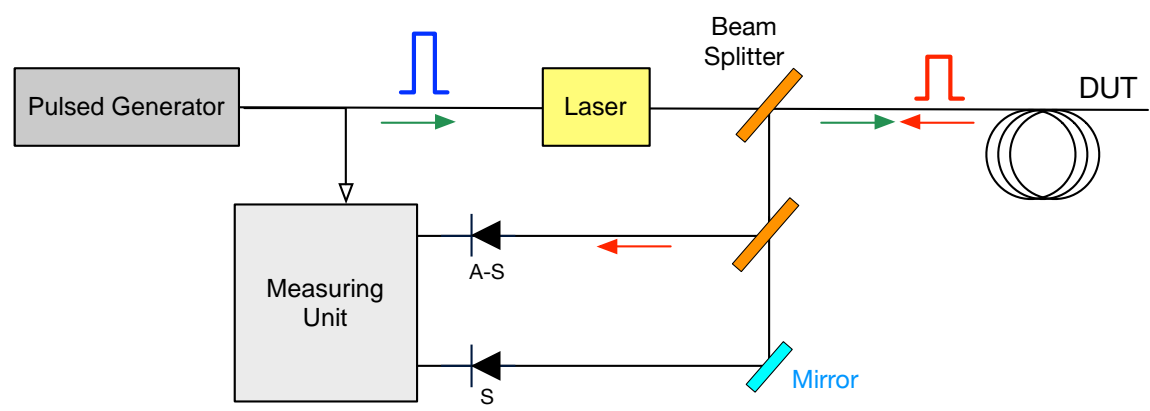

Figure 28. Principle of measurement of a DTS system (Hurtig, Großwig, \& Kühn, 1996).

Generally, the technique that uses Raman backscattered light to perform a continuous profile along the entire fibre length is called distributed temperature sensors (DTS) (Brown \& Hartog, 2002).

\subsubsection{Raman applications}

The Raman technique is mainly used for temperature measurement of civil structures such as oil pipes and electrical networks as well as leakage monitoring of large structures. Hence, in the literature can be found the DTS system to monitor the temperature in tunnels for fire detection (Ishii, Kawamura, Ono, Megumi, \& Kikkawa, 1997) or the temperature of injection well (Hurtig et al., 1996). This technique has also been used in a small scale to monitor the 
curing of epoxy resins in situ (Lyon, Chike, \& Angel, 1994). However, in terms of damage detection, smaller number of applications have been conducted using this technology. Hence some experiments can be found such as Gonzalez-Chi, Flores-Johnson, Carrillo-Baeza, \& Young (2010) that studied the axial stress around the kink bans, which may reduce the strength of the laminates, generated in a thermoplastic composite model.

Despite the high temperature accuracy and sensing range of this technique, the limitation in spatial resolution make it almost unsuitable for structural health monitoring in aerospace structures.

\subsubsection{Pipe Temperature Monitoring by Raman, OBR and FBG}

The experiment here presented was carried out to probe and test the Raman technology by the CCMSS at Puertollano solar thermal power plant. In this plant, the solar radiation is concentrated by means of parabolic mirrors which is then trapped by collector pipelines. This application aims to monitor the temperature of these pipelines in order to detect leaks at the earliest possible stage.

When a leak of a pipeline occurs, in most cases, it becomes difficult to detect and locate the event since these pipelines are in the order of tens of kilometres. Also, when this happens, the power plant should be closed resulting in high costs of maintenance and closure. Therefore, the possibility to obtain a distributed temperature measurement over the whole length of the pipeline offers significant benefits in terms of cost and maintenance time.

As has been proved, the main requirement of this application lies in the sensing range capability of the distributed measurement. Thereby, Rayleigh technique is not the most convenient technique since the sensing range is limited to 70 metres. Likewise, Brillouin technique is able to obtain better sensing range than Rayleigh technique in the order of hundreds of kilometres, but the strains are also coupled to the measurement and the spatial resolution achieved is around 5 metres (Angulo-Vinuesa et al., 2012). However, Raman technique can offer same sensing range with a resolution of 1 metre and it is only temperature sensitive. For such reasons, in this application, the most suitable proposal was to use the Raman technique.

Despite Raman technique, other different fibre optic sensors were installed to monitor the temperature of a pipe section. Therefore, besides the fibre optic line used for Raman measurement, a polyimide SMF fibre optic line was placed around the section of the pipe without bonding so as to avoid coupling of strains. Additionally, two FBGs were located at the upper and lower point of the pipe section. The schema of the configuration set-up with the different fibre optic sensing techniques is shown in Figure 29. 

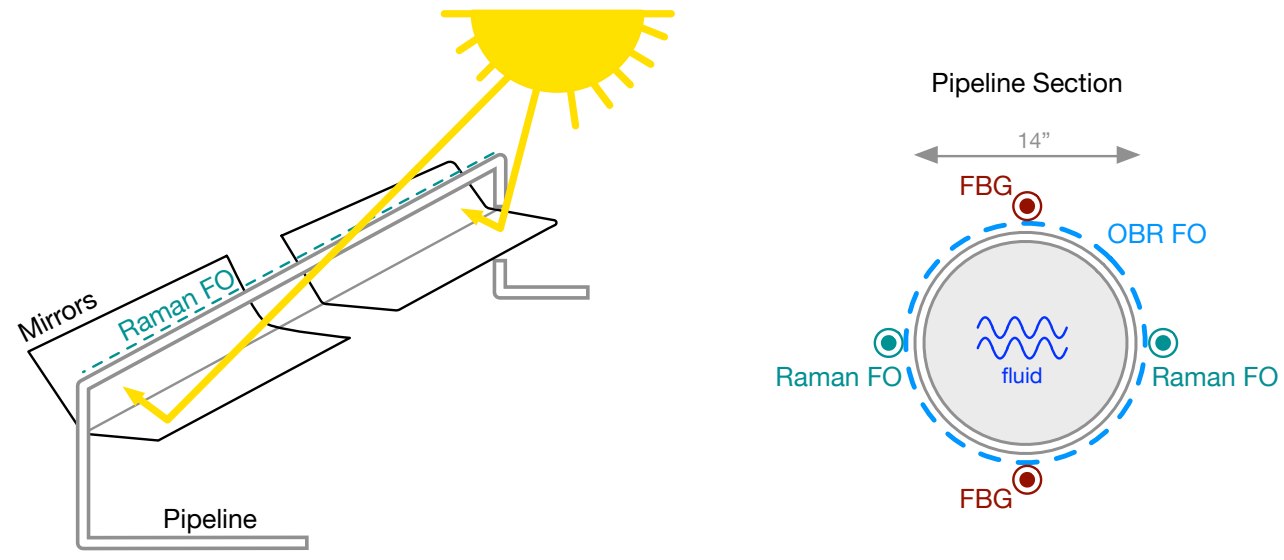

Figure 29. Setup of experimental distributed temperature measurement.

The pipe cross-section had a diameter of 14 inches of diameter, which makes a perimeter of 2.24 meters. The installation of the fibre optic line for Raman temperature measurement was performed in one of the rows of the power plant over a length of $20 \mathrm{~m}$. For this purpose, the coating of the pipe was partially removed for the installation of the sensors and then reinstalled to perform the experimental test. The test consists of a first part for temperature monitoring as long as the fluid flows over the pipeline by the different sensors (FBG, OBR and Raman). Then, a second part for temperature monitoring for one month only by means of Raman measurements.

The Raman system used to monitor the temperature was a Halo-DTS by Sensornet ${ }^{\circledR}$. The principle of this system is based on recording the Stokes and Anti-Stokes components of Raman backscattering from which the distributed temperature measurement is obtained. The frequency rate was fixed to $0.1 \mathrm{~Hz}$ with a spatial resolution of $2 \mathrm{~m}$.

Figure 30 shows the data that is included in a single measurement of the Halo-DTS system. Here, the distributed temperature measurement along the length of the fibre can be appreciated. The two points of the measurement located at length of $50 \mathrm{~m}$ had a lower readout since this section is outside of the pipeline coating at ambient temperature. Then, the fibre line return into the coating to measure the opposite side of the section. 

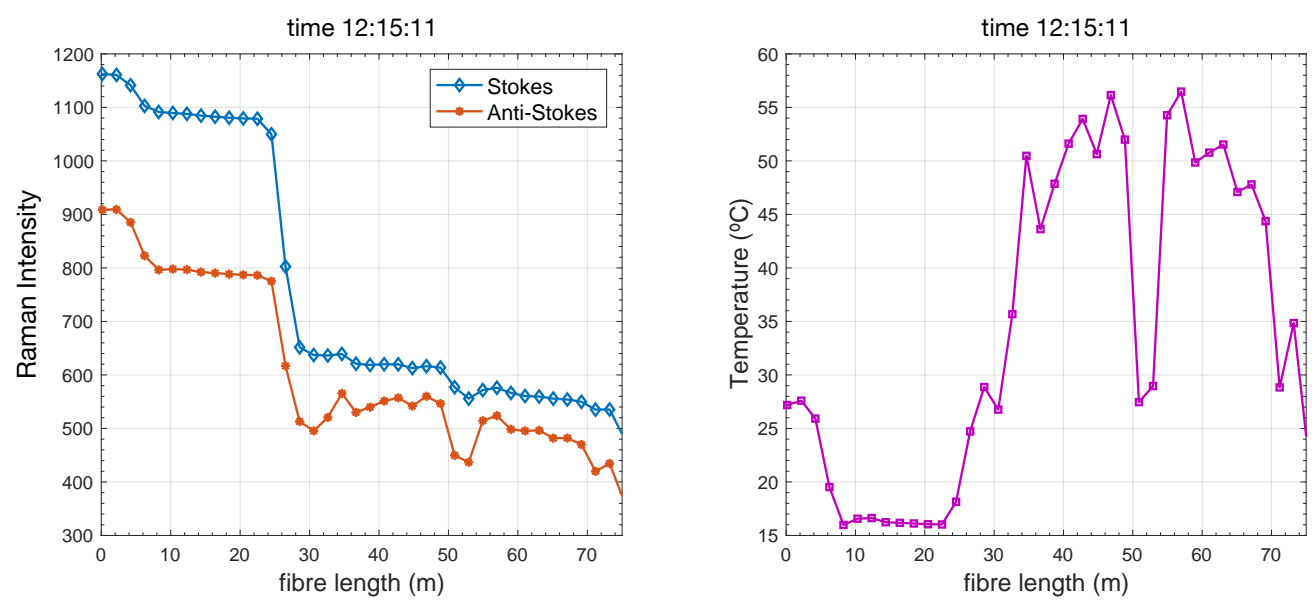

Figure 30. Single measurement acquired by Halo-DTS. (Left) Stokes and Anti-Stokes components of Raman scattering.

In Figure 31, the results of the distributed OBR measurement are presented for $251^{\circ} \mathrm{C}, 188$ ${ }^{\circ} \mathrm{C}, 22{ }^{\circ} \mathrm{C}, 244^{\circ} \mathrm{C}$ and $251^{\circ} \mathrm{C}$ regarding the thermocouple readout. As can be seen in this figure, there some areas with high temperature gradients. The origin of these changes is not well known since it may be induced by the coupling of the strain field or even by the temperature gradients around the pipeline section.

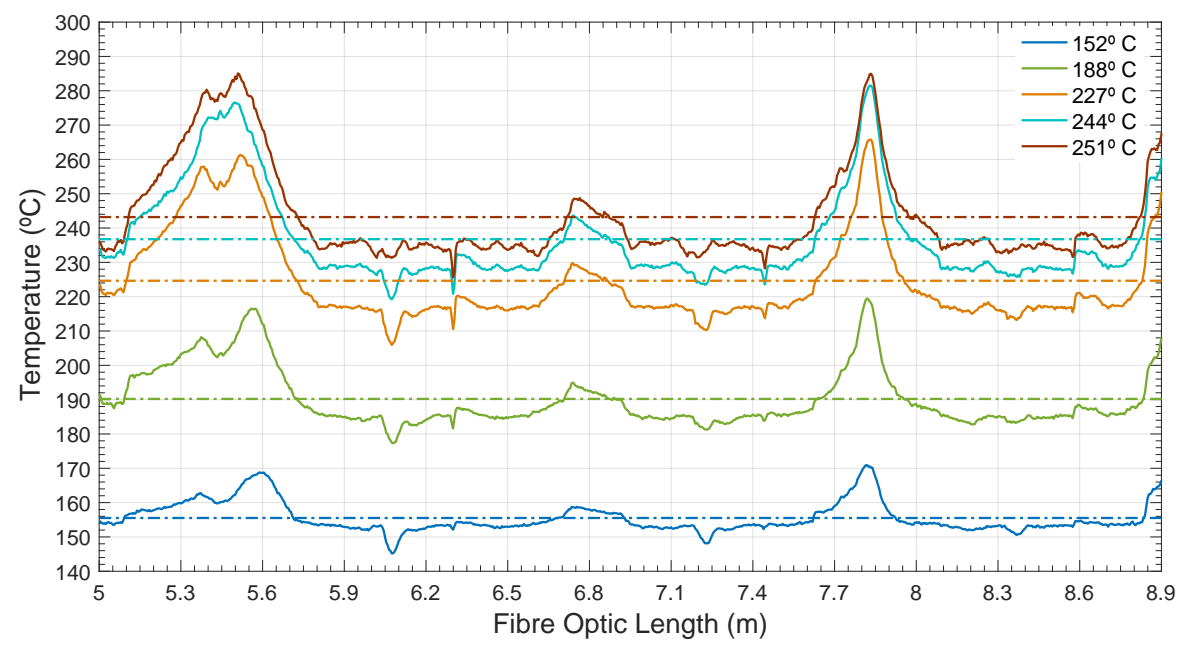

Figure 31. Distributed temperature measurement by OBR system. The dashed line is the average of the distributed measurement.

Figure 32 shows the comparison of the results obtained for all the FOS techniques installed in the pipeline. In view of the temperature results, FBG and OBR measurements show a fitting agreement at the steady temperature state. Also, some differences at the beginning of the fluid inlet can be appreciated. These variations are mainly due to the temperature transfer between the sensor and the pipeline. Therefore, a better agreement was obtained when all fluid was loaded at a steady temperature state. It should be noted that in order to ensure the 
correct temperature measurement without strain influence each FBG was embedded into a non-deformable tube. Regarding the Halo-DTS measurement, a good agreement was reached in the same manner. The differences between the measurements can be also explained by the temperature transfer. Whereas the FBG and OBR measurements were directly in contact with the surface of the pipeline, the fibre of the Halo-DTS had its own cladding and coating that was fixed to the pipeline by means of flanges. Another fact is, both FBG and OBR can detect strains, despite the measures taken, due to the thermal expansion of the pipeline.

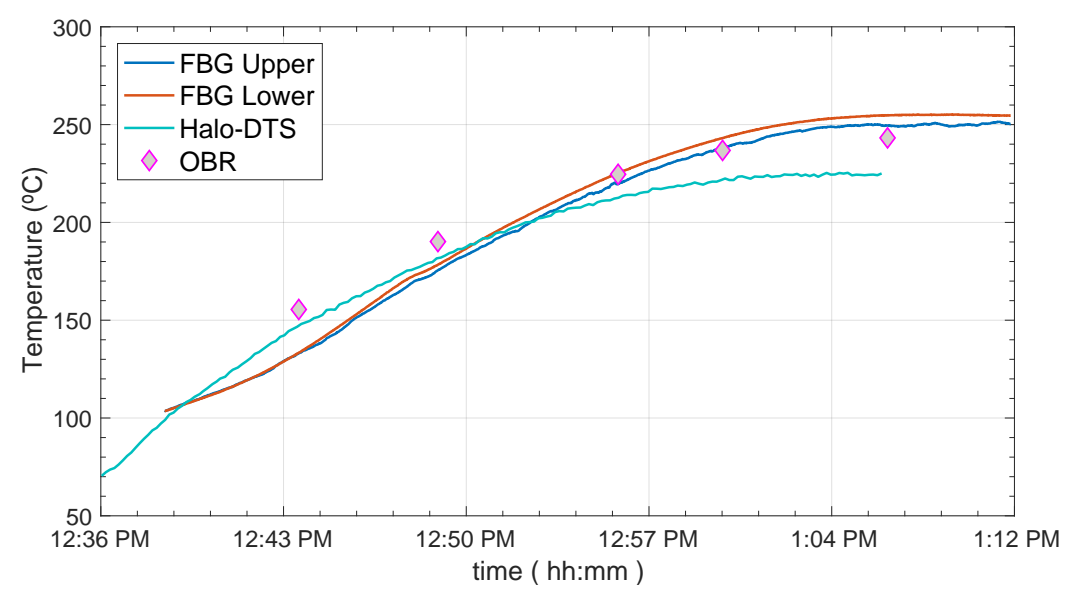

Figure 32. Temperature-time histories for the FBGs placed at lower and upper section points, for the Halo-DTS and for the OBR.

From this experiment the effectiveness of the choice of the Raman technique to monitor the temperature in large structures was demonstrated. Only dozens of meters were monitored to be able to compare the results with the other FOS techniques proposed. FBG and OBR return accuracy results with high spatial resolution but such a level for this application was not necessary. Moreover, a certain level of distrust appears to consider the thermal expansion of the pipeline and the coupling of the strain in the measurements. Nevertheless, by using Halo-DTS, similar temperature results are obtained without considering the expansion effect. Finally, the sensing range is a limiting requirement to consider Raman as the most suitable technique for this application.

\subsubsection{Distributed Sensors Based on Rayleigh Scattering}

Rayleigh scattering is a light feature that occurs in almost all mediums as a result of the interaction between particles much smaller than the propagation light wavelength.

Particularly, in optical fibres, the Rayleigh backscatter is a random but static feature of the light when it travels down to the core. This property can be understood as a repeatable scatter pattern and it is unique in each fibre. The origin of this pattern occurs in the manufacturing of the optical fibres, when small homogeneities are randomly created inside the core of the fused silica. Thereby, in general terms, when light is propagated through the 
optical fibre core, these small homogeneities change the reflectivity pattern of the light. This pattern is the so-called DNA of the optical fibre.

A schema of the formation of the reflectivity pattern is shown in Figure 33. The interaction of the light with the inhomogeneities of the optical fibre core result in a backscattering light which are strongly dependant of the propagation light wavelength with relation to $\propto \lambda^{-4}$ (Fang et al., 2012). Therefore, the longer wavelength used, the smaller intrinsic loss recorded. Therefore, the clearest example of this behaviour is found in the blue colour of the sky since the Rayleigh scattering of the shorter wavelength $(492-455 \mathrm{~nm})$ is much stronger.

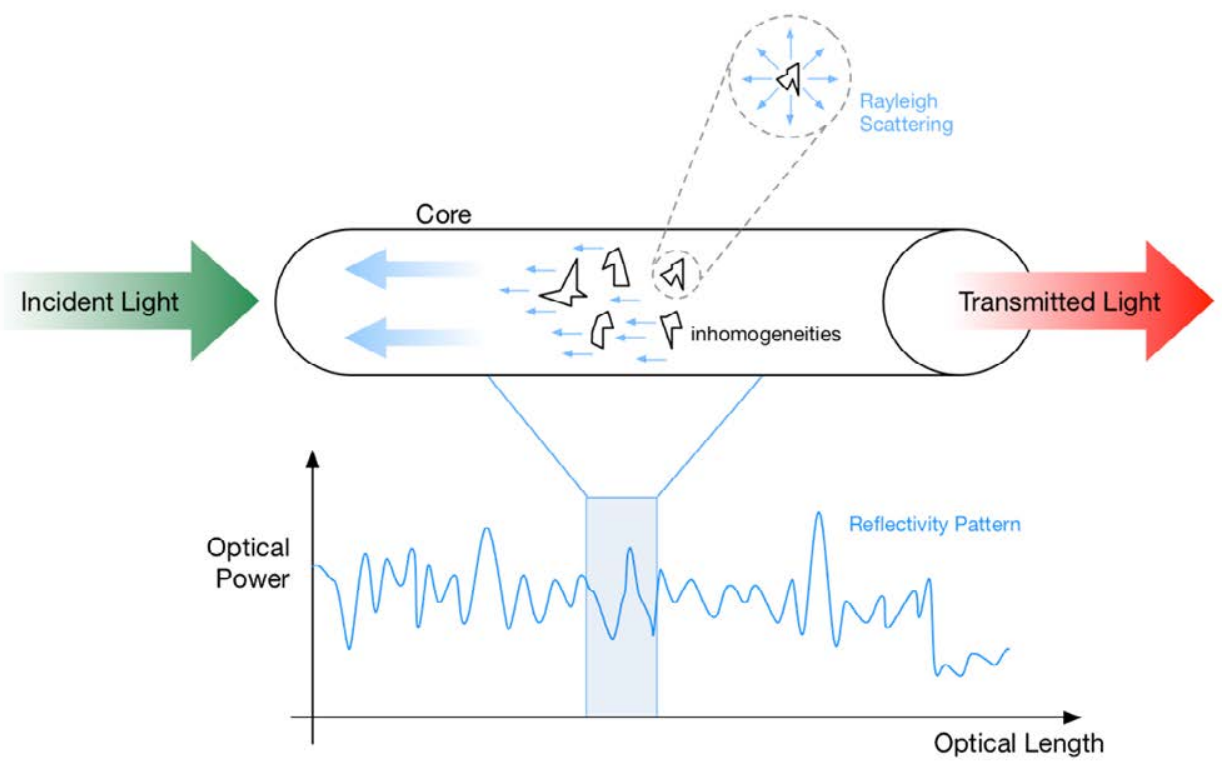

Figure 33. Formation of the reflectivity pattern in an optical fibre based on Rayleigh backscattering.

The measurement theory through Rayleigh technique is deeply described in Chapter 3.

\subsubsection{Rayleigh applications}

The first Rayleigh-based system was developed to evaluate the losses of an optical fibre, as described in 2.1. Since the OFDR based on Rayleigh backscattering was settled several and assorted experiments were carried out.

As occurred with the other distributed sensing techniques, the application fields are wide. Thereby, in the nuclear field, the temperature of a pool-type reactor was successfully monitored in order to indicate possible catastrophic failures (Sang, Froggatt, Gifford, Kreger, \& Dickerson, 2008). Furthermore, Rayleigh has been also used in civil structures. Therefore, a reinforced concrete slab was used to prove the effectiveness of this technique by monitoring the high strain concentrations due to cracks (Guemes et al., 2010). Also, in this operation, a wind turbine blade of $43 \mathrm{~m}$ long was monitored to detect the initiation of elastic buckling. In the field of aircraft structures, the work carried out by Güemes \& Fernandez-lopez (2010) 
should be regarded, to detect local cracks in bolted joints through distributed strain measurement.

Up to now, one of the most common applications for distributed Rayleigh-based technique is for structural test monitoring. This is mostly due to large sensing points that can be achieved with just a single optical fibre in a straightforward installation. In a local approach, the shear of a single lap joint was able to measure by means of a single optical fibre, even when a debonded area was induced in the adhesive (Grave, Håheim, \& Echtermeyer, 2015). Also, during the conduct of this thesis, different structural tests were put into effect. The experimental test was carried out in three composite stiffened panels to detect the appearance of potential failure points under compressive loads should be pointed out (Patricia; F.Díaz-Maroto, Fernández-Lopez, \& Güemes, 2015).

More examples of applications of Rayleigh-based technique will be presented in the results section (Chapter 5 and Chapter 6).

\subsubsection{DFOS comparison}

Following Table 2 establishes a comparison among the main different distributed techniques hereabove mentioned. It should be noted out that these performances can differ from one system to another for different techniques, but an overall information can be extracted from this table. Moreover, the references of these values are provided. 
Table 2. Distributed fibre optic techniques summary.

\begin{tabular}{|c|c|c|c|c|c|c|c|c|}
\hline & Measurand & $\begin{array}{c}\text { Strain } \\
\text { Resolution } \\
{[\mu \varepsilon]}\end{array}$ & $\begin{array}{c}\text { Temperature } \\
\text { Resolution } \\
{\left[{ }^{\circ} \mathrm{C}\right]}\end{array}$ & $\begin{array}{c}\text { Spatial } \\
\text { Resolution } \\
{[\mathrm{m}]}\end{array}$ & $\begin{array}{c}\text { Sensing } \\
\text { Range } \\
{[\mathrm{km}]}\end{array}$ & $\begin{array}{c}\text { Rate } \\
{[\mathrm{s}]}\end{array}$ & Suppliers & Sources \\
\hline $\begin{array}{l}\text { OTDR } \\
\text { (Rayleigh) }\end{array}$ & $\mathrm{T}, \varepsilon$ & $\mathrm{n} / \mathrm{a}$ & $\mathrm{n} / \mathrm{a}$ & 0.5 & $1-2$ & $<0.001$ & $\begin{array}{c}\text { NTEST, EXFO, } \\
\text { Viavi, VeEX, } \\
\text { AFL, ... }\end{array}$ & $\begin{array}{l}\text { (Eickhoff \& } \\
\text { Ulrich, 1981) }\end{array}$ \\
\hline $\begin{array}{l}\text { OFDR } \\
\text { (Rayleigh) }\end{array}$ & $\mathrm{T}, \varepsilon$ & 1 & 0.1 & 0.01 & 0.07 & 6 & $\begin{array}{c}\text { Luna } \\
\text { Technologies }\end{array}$ & $\begin{array}{c}\text { (Luna } \\
\text { Technologies } \\
\text { Inc., 2013) }\end{array}$ \\
\hline $\begin{array}{l}\text { OTDR } \\
\text { (Raman) }\end{array}$ & $\mathrm{T}$ & $\mathrm{n} / \mathrm{a}$ & $<1$ & $1-2$ & 20 & $\mathrm{n} / \mathrm{a}$ & $\begin{array}{l}\text { Halliburton } \\
\text { Co., AP } \\
\text { Sensing, } \\
\text { Sensornet }\end{array}$ & $\begin{array}{c}\text { (Glisic, 2013; } \\
\text { Güemes et al., } \\
\text { 2018) }\end{array}$ \\
\hline BOTDA & $\mathrm{T}, \varepsilon$ & 2 & 0.1 & 1.5 & 60 & 600 & $\begin{array}{c}\text { OZ Optics, } \\
\text { Smartec, } \\
\text { Neubrex }\end{array}$ & $\begin{array}{l}\text { (Schenato, } \\
\text { 2017) }\end{array}$ \\
\hline BOTDR & $\mathrm{T}, \varepsilon$ & 0.1 & 0.005 & 80 & 45 & 1800 & $\begin{array}{c}\text { OZ Optics, } \\
\text { Neubrex, } \\
\text { NTT, Smartec }\end{array}$ & $\begin{array}{l}\text { (Schenato, } \\
\text { 2017) }\end{array}$ \\
\hline BOFDA & $\mathrm{T}, \varepsilon$ & 30 & 1.8 & 3 & 0.009 & $\mathrm{n} / \mathrm{a}$ & FibrisTerre & $\begin{array}{c}\text { (Bernini, } \\
\text { Minardo, \& } \\
\text { Zeni, 2012) }\end{array}$ \\
\hline $\begin{array}{l}\text { OFDR } \\
\text { (FBG) }\end{array}$ & $\mathrm{T}, \varepsilon$ & 5 & $\mathrm{n} / \mathrm{a}$ & 0.001 & 0.02 & $1 / 150$ & $\begin{array}{c}\text { Lazoc, } \\
\text { FemtoFiberTec }\end{array}$ & $\begin{array}{c}\text { (Igawa et al., } \\
\text { 2008; Wada, } \\
\text { Igawa, } \\
\text { Murayama, \& } \\
\text { Kasai, 2014) }\end{array}$ \\
\hline
\end{tabular}




\title{
Chapter 3 MEASUREMENT THEORY OF OPTICAL BACKSCATTER REFLECTOMETER
}

\begin{abstract}
A detailed description of the measurement theory for Optical Backscatter Reflectometer, an OFDR system, is put into practice throughout this chapter for a better understanding of this technique. Furthermore, some of the presented results have been analysed by means of some specific algorithms.
\end{abstract}

\subsection{Introduction}

After presenting the different distributed sensing techniques, the OFDR Rayleigh-based was selected to obtain the distributed measurement. In this field, the Optical Backscatter Reflectometer 4600 is probably the most competitive and advanced system in the fibre optics market.

This system was introduced in 2004 by Luna Innovations and, from that moment, it supposed an important step forward into the fibre optic sensing field. At the time, OBR was the most sensitive commercial system $(-130 \mathrm{~dB})$ with the highest strain and temperature resolution (1 microstrain and $0.1^{\circ} \mathrm{C}$ ) (Froggatt \& Moore, 1998b; Soller, Wolfe, et al., 2005).

The theory behind the distributed measurement technique deserves to be discussed, not only for a better understanding, but also to get the most from this technology.

\subsection{General OBR4600 Functioning}

The Optical Backscatter Reflectometer system enables to measure the Rayleigh scattering which, as explained in section 2.5.3, is produced as a result of random fluctuations in the local refractive index occurred during the manufacturing of the optical fibres. OBR belongs to the OFDR type system that uses swept-wavelength interferometry. Therefore, through a tuneable laser the device under test, i.e. optical fibre, is interrogated with a high spatial resolution and accuracy.

The laser sweeps with equal frequency intervals in order to obtain a measurement of the total complex reflectivity along the device under test (DUT). This measurement is given by the vector sum of the two orthogonal polarization states, i.e. primary ('p') and secondary 
mode ('s'). By means of this complex sum in the frequency domain, the reflectivity pattern as a function of the fibre length is calculated through a fast Fourier transform (FFT) (Soller, Gifford, et al., 2005).

Two measurements of the reflectivity pattern are needed to obtain the strains and/or temperature differences between both states. For this purpose, one measurement is considered as a reference signal and the other measurement acts as a loaded signal. It should be noted that the reflectivity pattern experiences a linear shift when a segment of the DUT is strained or heated. Then, the experienced spectral shift is linearly dependant on the temperature $(\Delta T)$ and strain $(\Delta \varepsilon)$ changes at each segment as shown in Equation 3-1. Here, $K_{T}$ and $K_{\varepsilon}$ are the temperature and strain coefficients, $\Delta \lambda$ and $\Delta v$ are the wavelength and frequency shift, and $\lambda_{0}$ and $v_{0}$ are the centre wavelength and frequency of the distributed measurement, respectively. In order to determine this spectral shift in frequency or wavelength domain, a cross-correlation between the two fibre states is performed (see Figure 34).

$$
\frac{\Delta \lambda}{\lambda_{0}}=-\frac{\Delta \nu}{v_{0}}=K_{T} \Delta T+K_{\varepsilon} \Delta \varepsilon
$$

A whole distributed measurement can be built when shifts are calculated sequentially along the fibre, i.e. by carrying out a cross-correlation for each of the DUT segments. It should be mentioned that reflectivity pattern is likely to become attenuated due to fibre optic loops and/or transverse stresses. In such a case, the calculation of the cross-correlation may turn out to be cumbersome (Patricia F. Díaz-Maroto, Fernández-López, García-Alonso, Iglesias, \& Güemes, 2018).

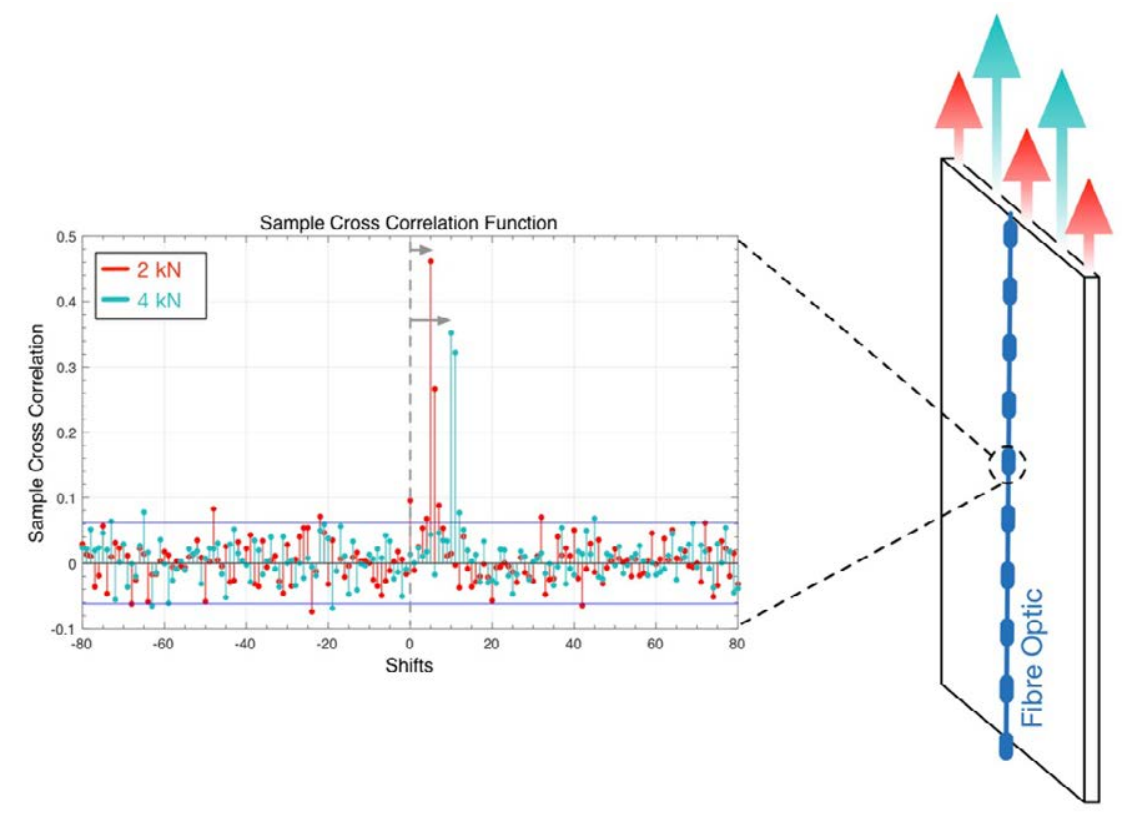

Figure 34. An aluminium coupon under $2 \mathrm{kN}$ and $4 \mathrm{kN}$ of tensile load. The number of spectral shifts can be obtained after cross-correlation against the reference measurement (Patricia F. Díaz-Maroto et al., 2018). 
An example is shown in Figure 34. This figure, shows the cross-correlation for a single point in a fibre optic line placed on an aluminium coupon (Patricia; F.Díaz-Maroto et al., 2015). In this case, the influence of the tensile load regarding the spectral shifts must be pointed out. As expected, the load of $4 \mathrm{kN}$ gives more number of shifts than the load of $2 \mathrm{kN}$. Then, the number of shifts can be converted into temperature and/or strain through the relationship given in Equation 3-1. In order to obtain a continuous distributed measurement, this operation is repeated as many times as the select section of the DUT.

\subsubsection{Technical specifications}

The Luna Technologies OBR 4600 is a Class 1 Laser Product. A summary of the OBR specifications is shown in Table 3 which are validated for single-mode operation. The commercial system is also shown in Figure 35.

Table 3. Summary of OBR 4600 performances $^{6}$.

\begin{tabular}{l|l|l}
\hline Parameter & Specifications & Units \\
\hline Optical Performances & & \\
\hline Maximum Device Length & 30 or 70 & $\mathrm{~m}$ \\
\hline Spatial Resolution (two-point) & 10 over 30 meters & $\mu \mathrm{m}$ \\
\hline Wavelength Range & 20 over 70 meters & $\mu \mathrm{m}$ \\
\hline Wavelength Resolution (max) & $1525-1610$ & $\mathrm{~nm}$ \\
\hline Wavelength Accuracy & 0.02 & $\mathrm{pm}$ \\
\hline Dynamic range & \pm 1.5 & $\mathrm{pm}$ \\
\hline Total range & 70 & $\mathrm{~dB}$ \\
\hline Sensitivity & 0 to -125 & $\mathrm{~dB}$ \\
\hline Resolution & -130 & $\mathrm{~dB}$ \\
\hline Accuracy & \pm 0.05 & $\mathrm{~dB}$ \\
\hline Group Delay Accuracy & \pm 0.10 & $\mathrm{~dB}$ \\
\hline Distributed Sensing Performances & 1.0 & $\mathrm{ps}$ \\
\hline Spatial Resolution & \pm 10 & \\
\hline Temperature Resolution & \pm 0.1 & $\mathrm{~mm}$ \\
\hline Strain Resolution & \pm 1 & $\mathrm{C}$ \\
\hline & & $\mu \mathrm{strain}$ \\
\hline
\end{tabular}

\footnotetext{
${ }^{6}$ http://lunainc.com/wp-content/uploads/2012/11/OBR-4600-Data-Sheet-9-Mar-2018.pdf
} 


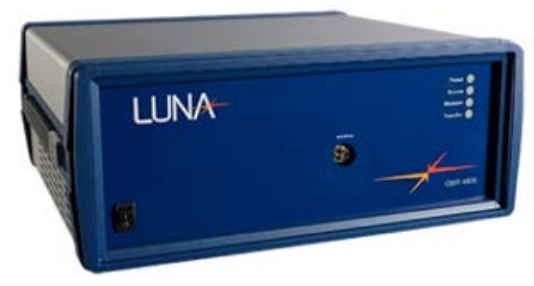

Figure 35. Commercial Luna OBR-4600 system.

\subsection{Optical Network Operation}

A functioning schema of an OBR is illustrated in Figure 36, where different optical devices and components can be distinguished:

- A tuneable laser to sweep the wavelength in equal intervals for a specific range. The typical scan range is from $1547 \mathrm{~nm}$ to $1590 \mathrm{~nm}$ to reduce the optical losses.

- 2 Mach-Zender interferometers. One of them is used as a reference to monitor the phase error during laser tuning, and the other interferometer is placed with the DUT.

- $3 \mathrm{~dB}$ couplers are used to recombine and split the light to the fibre optical paths.

- A beam splitter is used to split the light in the two orthogonal polarization modes.

- A detector receives the two polarization modes and the reference measurement.

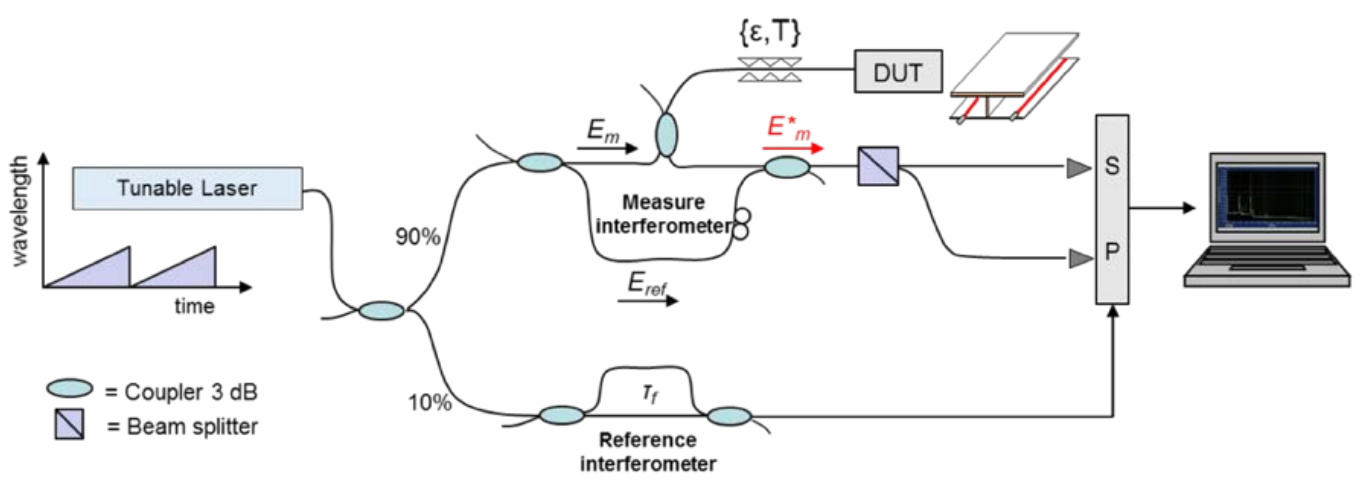

Figure 36. Functioning schema of an OBR.

\subsection{Interference Pattern Acquisition}

A simplified OBR system schema is shown in Figure 37. As explained above, the three basic components are a tuneable laser source (TLS), a Mach-Zender interferometer and a detector. The TLS allows to control the wavelength scan range and the scan rate. Then, the optical fibre network to monitor, which is expected to be under tensile, compressive or temperature loads, is a so-called device under test. As can be seen in the figure, the DUT is placed into one of interferometer arms. 


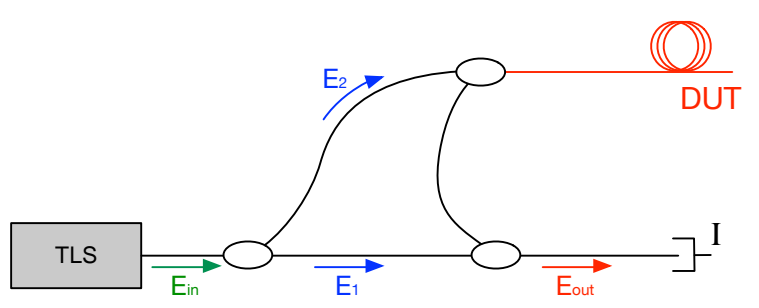

Figure 37. Simplified OBR system schema. Three basic components: TLS, DUT and Mach-Zender interferometer.

The TLS continuously sweeps the interrogation frequency. Therefore, in an instant $t$ the TLS sweeps in an instantaneous frequency $\omega(t)$. Then, the electric input $\left(E_{\text {in }}\right)$ generated can be expressed in terms of the two optical paths $E_{1}$ and $E_{2}$ of the interferometer (see Equation 3-2).

$$
E_{\text {in }}=E_{0}(t) e^{-i \omega(t) t}=E_{1}(\mathrm{t})+E_{2}(t)
$$

Once $E_{1}$ and $E_{2}$ travels along its paths, the light is recombined again in the coupler to record $E_{\text {out }}$. As expected, the output light from both paths has experienced different delays, which are denoted by $\tau_{1}$ and $\tau_{2}$. The electrical output, $E_{\text {out }}$ can be expressed by the Equation 3-3.

$$
E_{\text {out }}=E_{0}\left(t+\tau_{1}\right) e^{-i \omega\left(t+\tau_{1}\right) t}+E_{0}\left(t+\tau_{2}\right) e^{-i \omega\left(t+\tau_{2}\right) t}
$$

Making the assumption that the electrical output which arrives at the detector is proportional to the square of the electric field, it can be expressed in the following Equation 3-4 and Equation 3-5.

$$
\begin{gathered}
I(\omega) \alpha<\mathrm{E}_{1} E_{2}>={E_{1}}^{2}+{E_{2}}^{2}+2 E_{1} E_{1} \cos (\omega(t) \tau) \\
I=\left|E_{0}(t)\right|^{2}+\left|E_{0}(t-\tau)\right|^{2}+2 E_{0}(t) E_{0}(t-\tau) \cos (\omega(t) \tau)
\end{gathered}
$$

where $\tau=\tau_{1}-\tau_{2}$ is the difference time delay between the two arms of the interferometer. The DUT, located in one arm of the Mach-Zender interferometer can be described by its frequency domain linear transfer function $H(\omega)$. This function, as mentioned, contains information about the phase $\phi(\omega)$ and the amplitude $\rho(\omega)$ of the light through the DUT. It can be expressed in the Equation 3-6:

$$
H(\omega)=\rho(\omega) e^{i \phi(\omega)}
$$

In these terms, the electrical input received in the detector can be written as an Equation 3-7: 


$$
I=\underbrace{\left|E_{0}(t)\right|^{2}+\left|E_{0}(t-\tau)\right|^{2} \rho(\omega)^{2}}_{\text {Low frequencyterms }}+\underbrace{2 \rho(\omega) E_{0}(t) E_{0}(t-\tau) \cos (\omega(t) \tau-\phi(\omega))}_{\text {Interference term }}
$$

Hence, it can be noted that the two first terms $\left|E_{0}(t)\right|^{2}+\left|E_{0}(t-\tau)\right|^{2} \rho(\omega)^{2}$ are low frequency terms, i.e., it appears close to $t=0$ in the time domain. However, the third term, called interference term, includes the DUT information since it can oscillate at the frequency $\omega(t) \tau$. In order to separate these terms a long delay between interferometer arms is provided by the OBR to ensure no interaction between them. These terms can be separated spectrally through a fast Fourier transform and the reflected optical intensity can be clearly located in the time domain.

Multiple reflections at different delays build the whole interference pattern of the optical fibre. Each of these reflections can be therefore interpreted as a peak in the time domain when they are Fourier transformed. Moreover, reflections peaks in the time domain can be straightforward expressed in terms of the DUT length by the relationship between vacuum light speed and the group index of the optical fibre.

Figure 38 shows the FFT of the transfer function at an instant frequency $\omega(t)$. One peak is located at the corresponding delay in the time-domain. This is only one reflection, and the whole interference pattern of the DUT is obtained by recording multiple delays in the whole frequency scan range.

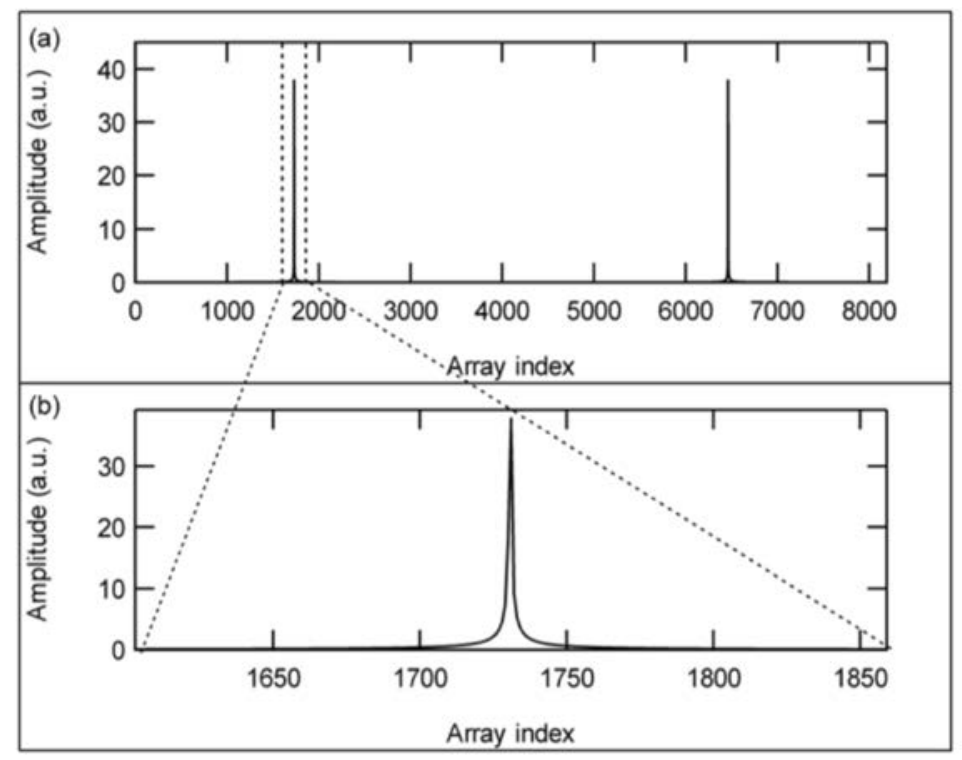

Figure 38. Reflection peak in time domain for an instant frequency (Luna Technologies Inc., 2013). 
Therefore, the impulse response in time domain can be calculated from the linear transfer function as shown in Equation 3-8.

$$
\tilde{h}_{j}=F F T\{H(\omega)\}_{j}
$$

Where $j$ corresponds to each location of the sensing points all along the DUT.

\subsubsection{Length Measurement}

The spatial resolution in terms of fibre optic length is constrained by the optical frequency range of the TLS. Then, the total time delay increment between two adjacent points of the DUT can be straightforward obtained as $\delta \tau=1 / \Delta v$, where $\Delta v$ is the total optical frequency scan range which can be calculated from the Equation 3-9 (Luna Technologies Inc., 2014). Where $S$ is the total number of sensing points that shape the distributed measurement and $\delta v_{T L S}$ and $\tau_{T L S}$ is the TLS frequency increment and TLS delay, respectively.

$$
\Delta v=S \delta v_{T L S}=\frac{S}{\tau_{T L S}}
$$

Therefore, in terms of fibre length the distance between two adjacent points can be obtained as expressed in Equation 3-10.

$$
\delta L=\frac{c \delta \tau}{2 n_{e f f}}=\frac{c}{2 n_{e f f} \Delta v}
$$

Where $c$ is the vacuum speed of light $\left(2.998 \times 10^{8} \mathrm{~m} / \mathrm{s}\right)$ and $n$ is the index of refraction.

For instance, if an OBR distributed measurement of a SMF $\left(n_{\text {eff }} \simeq 1.47\right)$ and the time increment $\delta \tau$ fixed at $9.54 \cdot 10^{5} n s$, the length increment between two adjacent points is 9.578 $\mu \mathrm{m}$. This is the distance at which an interference fringe is collected and analysed. It should be noted that OBR measurement always presents the same number sampling points for each of the two allowing interrogation lengths $(35 \mathrm{~m}$ or $70 \mathrm{~m}$ ) in order to keep the spatial resolution independent of the recorded points $S$.

\subsection{Group Delay and Polarization Mode Dispersion}

In previous chapters, the light scattering into the optical fibres have been meticulously introduced. Hereafter, the polarization mode dispersion (PMD) in optical fibres is presented.

Ideally, if the cross-section of an optical fibre is perfectly circular, there are two orthogonal polarization modes (as a consequence of electric field orientation) which travel at the same velocity. In a real case, the symmetry of the fibre core is broken, and birefringence appears. 
Then, two different orthogonal modes are scattered when they are propagated through the optical fibre. This is the so-called polarization mode dispersion. Therefore, under PMD the orthogonal modes travel with different velocities. It must be called to attention that, despite the polarization being randomly allocated, it is a constant feature for each optical fibre.

As explained in previous chapters, this dispersion is also caused by random inhomogeneities and asymmetries present in the core of the optical waveguide which induce changes in the refractive index. Not only is the birefringence produced by intrinsic factors in the core due to fibre optic manufacturing, but also it can be caused by external factors such as: external loads, temperature changes, fibre twisting or bending.

Another term should be mentioned: the differential group delay (DGD). This is the propagation time difference between both polarization modes (see Figure 39).

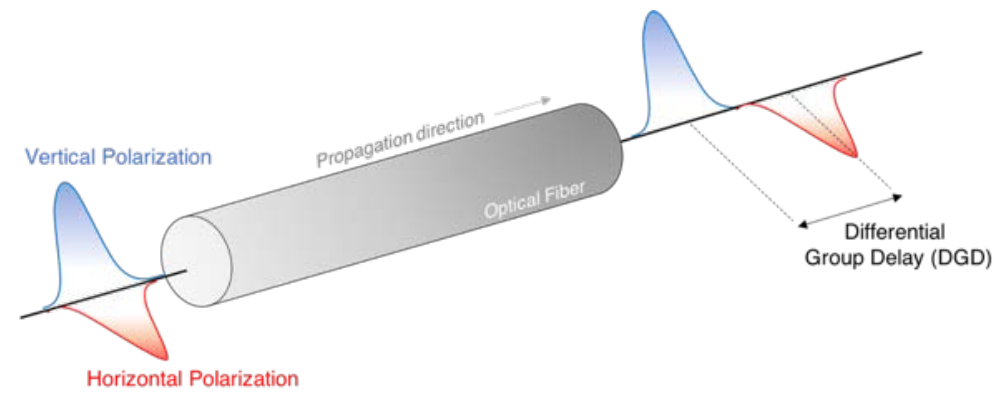

Figure 39. Differential Group Delay in an optical fibre.

In the OBR system, the DGD is understood as the phase changes in terms of frequency. It can be obtained from the signal delay of the modulated optical sinusoidal envelope when it is propagated through the optical fiber (Luna Technologies Inc., 2013). In more detail, the group delay (GD) at every sensing point of the fiber is calculated as:

$$
G D_{i}=\frac{\arg \left(H_{i+1} H_{i}^{*}\right)}{\Delta \omega}
$$

Where $\arg (x)$ is the argument of the complex number $x, H$ is the transfer function and $\Delta \omega$ is the frequency increment between samples.

\subsubsection{Orthogonal Polarization Modes 's' and 'p'}

As introduced in the previous section, the light is split into two orthogonal polarization states as a consequence of the electric field orientations. In the OBR system, these two polarization modes are recorded separately. Then, the data points from primary (mode ' $\mathrm{p}$ ') and secondary (mode ' $s$ ') is obtained in a complex form expressed in Equation 3-12.

$$
r_{p}=\rho(\omega) e^{i \phi(\omega)}=\rho(\omega)+i \phi(\omega)
$$


Where $\rho(\omega)$ is the amplitude and $\phi(\omega)$ is the phase for an instant frequency $\omega$. Also, this data can be obtained in time domain as shown in Equation 3-13 (Soller, Gifford, et al., 2005):

$$
r(\tau)=\sqrt{\left|\widetilde{\iota_{s}}(\tau)\right|^{2}+\left|\widetilde{\iota_{p}}(\tau)\right|^{2}}
$$

Where $\widetilde{\imath_{s}}(\tau)$ and $\widetilde{\imath_{p}}(\tau)$ is time-domain response of the DUT, i.e. primary and secondary modes respectively.

In Figure 40, the two orthogonal polarization states of a fibre optic were extracted to perform the total reflectivity pattern. As observed in this plot, the DUT section under strain and/or temperature, both modes sharply change, whereas in the rest of the fibre the polarization modes remain similar. The same behaviour is appreciated along the length of the fibre which is contained in the FO switch. The reflections due to the FC/APC are also noticeable.
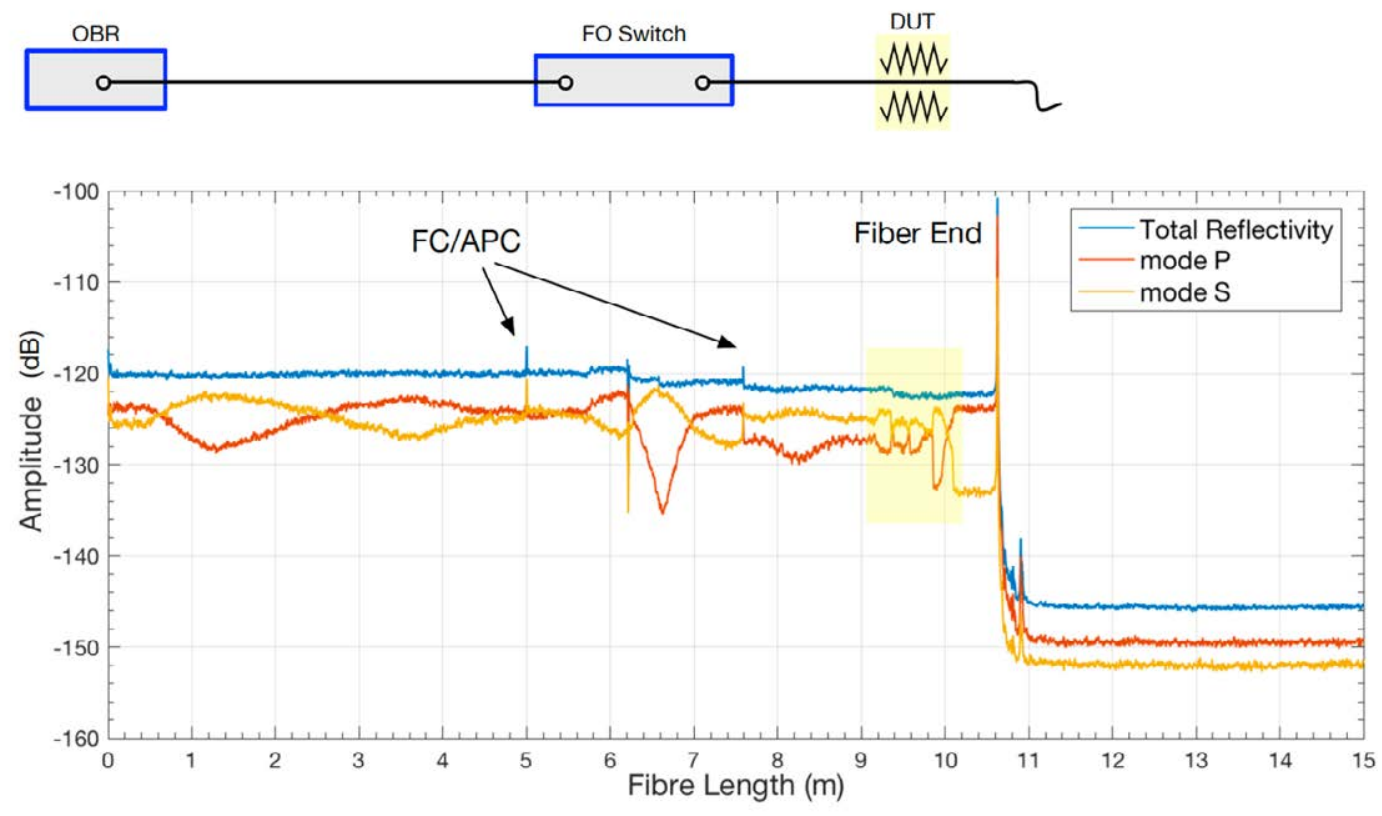

Figure 40. Polarization states in time domain in a real measurement acquired by the OBR. The measurement has been obtained by a moving average of 1000 points.

\subsection{Distributed Measurement Sensitivity}

The sensitivity of the distributed measurement acquired by the OBR can be presented through an experimental test to monitor the crystallisation of a ring-opening thermoplastic in a resin transfer moulding (RTM) process. In this experiment, the amplitude of the backscattering recorded by the optical fibre decrease provides information about the state of the resin crystallisation in a qualitative way. Then, the total crystallization corresponds to the lowest backscattered amplitude. 
The mould and plain weave fabric of glass with the optical fibre path is shown in the Figure 41. The fibre had polyimide coating in order to protect itself against the high temperatures of resin injection. But it was fixed to the last layer with cyanoacrylate. Small radius in loops can also be distinguished in this figure by means of the red light of the fibre checker.
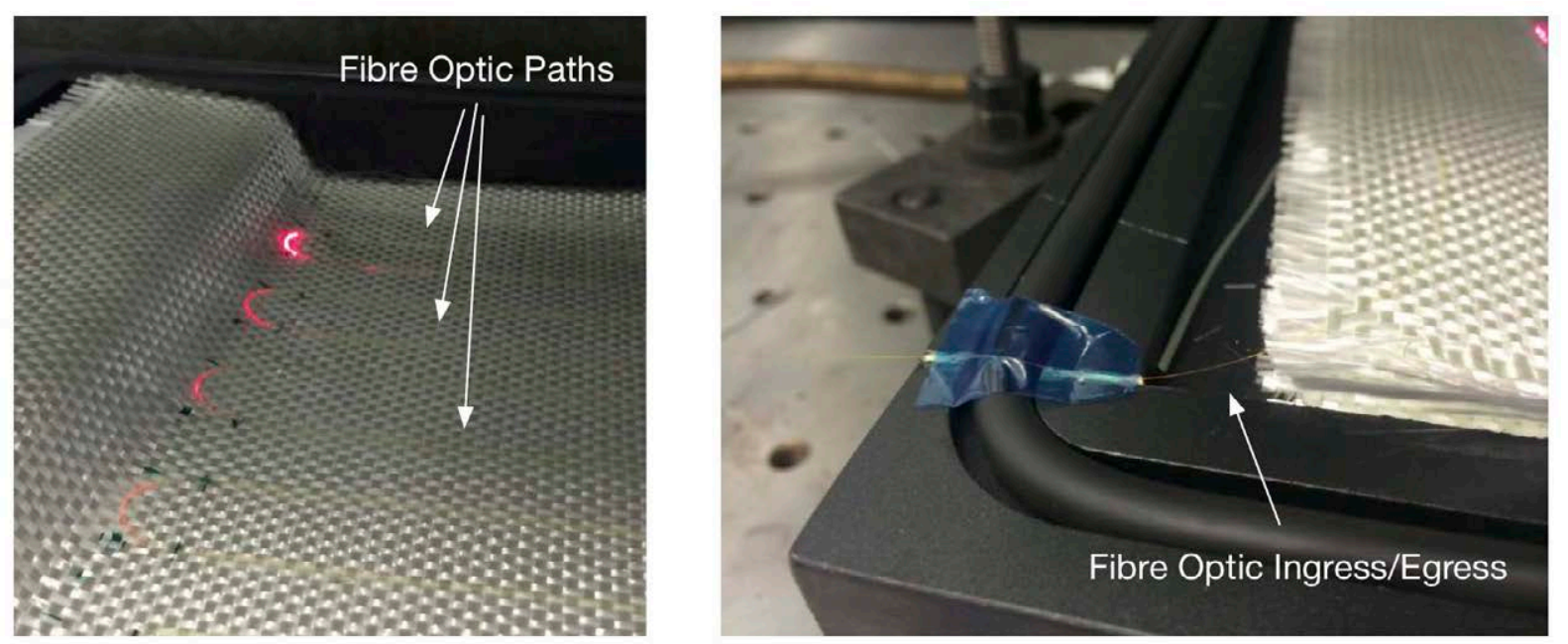

Figure 41. Experimental setup for the crystallisation monitoring.

As optical losses are proportional to the transversal stresses in every point of the fibre, it is possible to detect the resin shrinkage due to crystallisation event in different paths of the fibre line (Ortiz de Mendibil, F. Díaz-Maroto, Fernández-Lopez, Aurrekoetxea, \& Güemes, 2014). In Figure 42, the temperature predicted by the amplitude of the distributed measurement was in between $190^{\circ} \mathrm{C}$ and $200^{\circ} \mathrm{C}$, showing a good agreement with the DSC test.
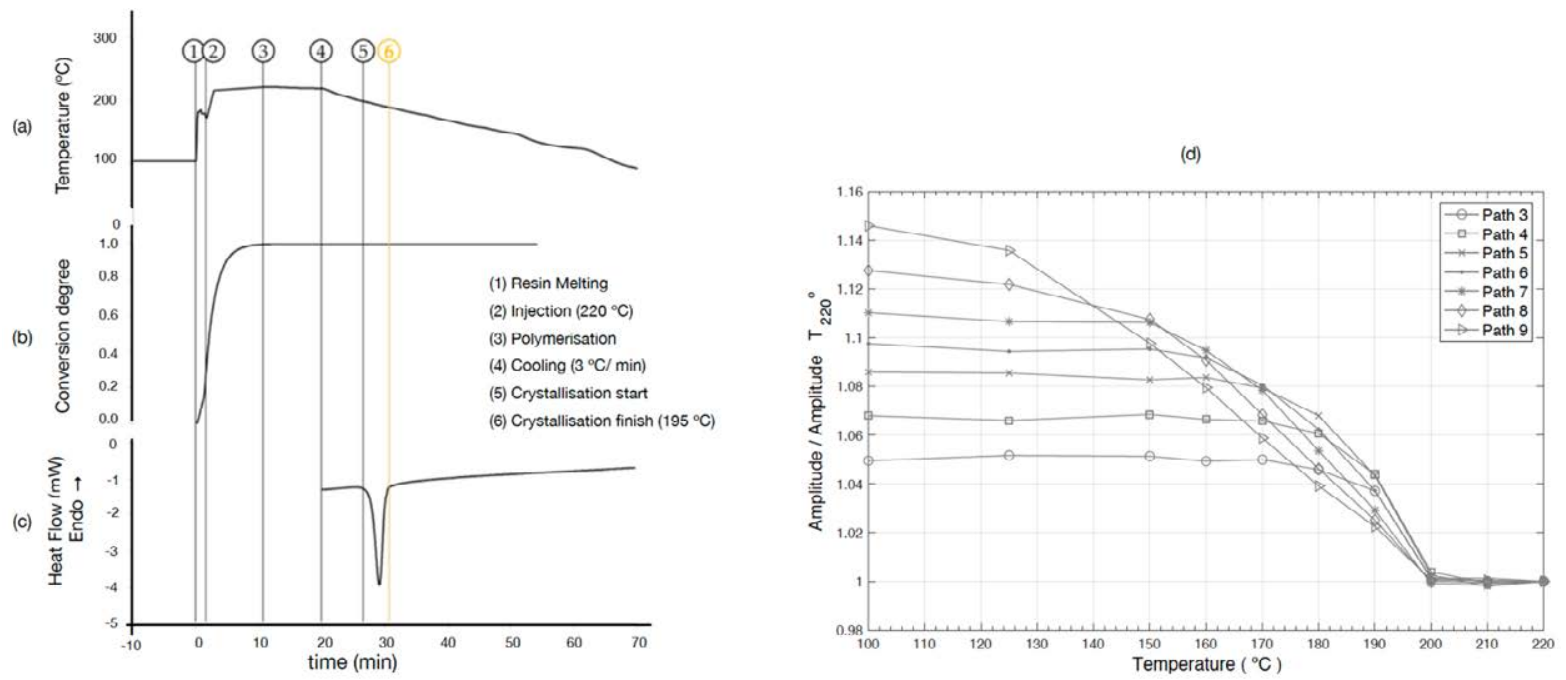

Figure 42. Results of the crystallisation experimental test. (a) Temperature, (b) Conversion degree, (c) DSC test, (d) fibre optic losses at different paths. 
Due to the high transversal stresses into the optical fibre, high optical losses were recorded under the sensibility of the system $(-130 \mathrm{~dB})$, see Table 3 . Hence, the strain measurement was not able to provide correctly the filling of the resin into the mould based on the strains. However, other characteristics of the measurement, such as the amplitude, could be exploited to monitor the point of the crystallisation finish. Throughout this test, the capability of monitoring by means of the backscattered light was demonstrated.

\subsection{Signal Conditioning Parameters}

Prior to obtain a distributed temperature or strain measurement, signal conditioning parameters have to be chosen by the user which leads to an important influence in the final distributed measurement.

Then, prior to calculating the cross correlation between the reference and the loaded measurement, the defined set of signal conditioning parameters are selected. These parameters should be evaluated and chosen regarding the sensing network features and the experimental set-up since this choice determines the accuracy and the resolution of the distributed strain measurement. As distributed measurements are strongly dependant on these parameters further explanation is presented in the next paragraphs:

- Gauge Length: this parameter corresponds to the sensor length. As explained in the measurement principle of the OBR, the spectral shift is obtained through the cross-correlation between two signals (reference and strained) included in the window width of the gauge length. On one side, the shorter the length used, the fewer the number of data points will be included in the spectral shift calculation and therefore, the signal might become unstable and with noise. On the other side, the calculation of spectral shift might be obtained with a longer sensor length, and consequently greater numbers of points will be used to obtain the spectral shift. In this case, where the structure is under small overload areas, its distributed measurement undergoes strain changes rapidly over short distances. If the sensor length is too long these overload areas (potential failure points) might be missed and undetected. Therefore, a good gauge length selection ensures a better temperature or strain accuracy and resolution (Patricia F. Díaz-Maroto et al., 2018).

- Sensor spacing: this parameter refers to the spacing between consecutive sensors. Depending on gauge length selection sensors might overlap. Three possibilities can be considered as shown in Figure 43.

- Sensing range: this parameter defines the optical fibre path of interest to perform the measurement, i.e. the starting and ending point of the sensing network over the structure. The longer the path selected, the more computational cost would be required. 


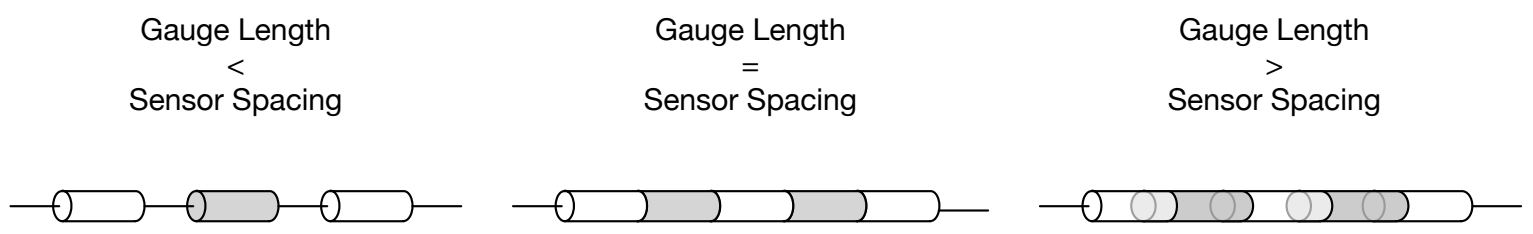

Figure 43. Gauge length and sensor spacing possibilities.

Next Figure 44 summarizes the signal conditioning parameters explained hereabove. An area is selected to analyse (red square) from the total reflectivity signal, i.e. the sensing range. Then, at every sensing point, the measurement points included in the gauge length are submitted under a cross correlation. Repeating this process for each sensing point the distributed measurement is obtained.
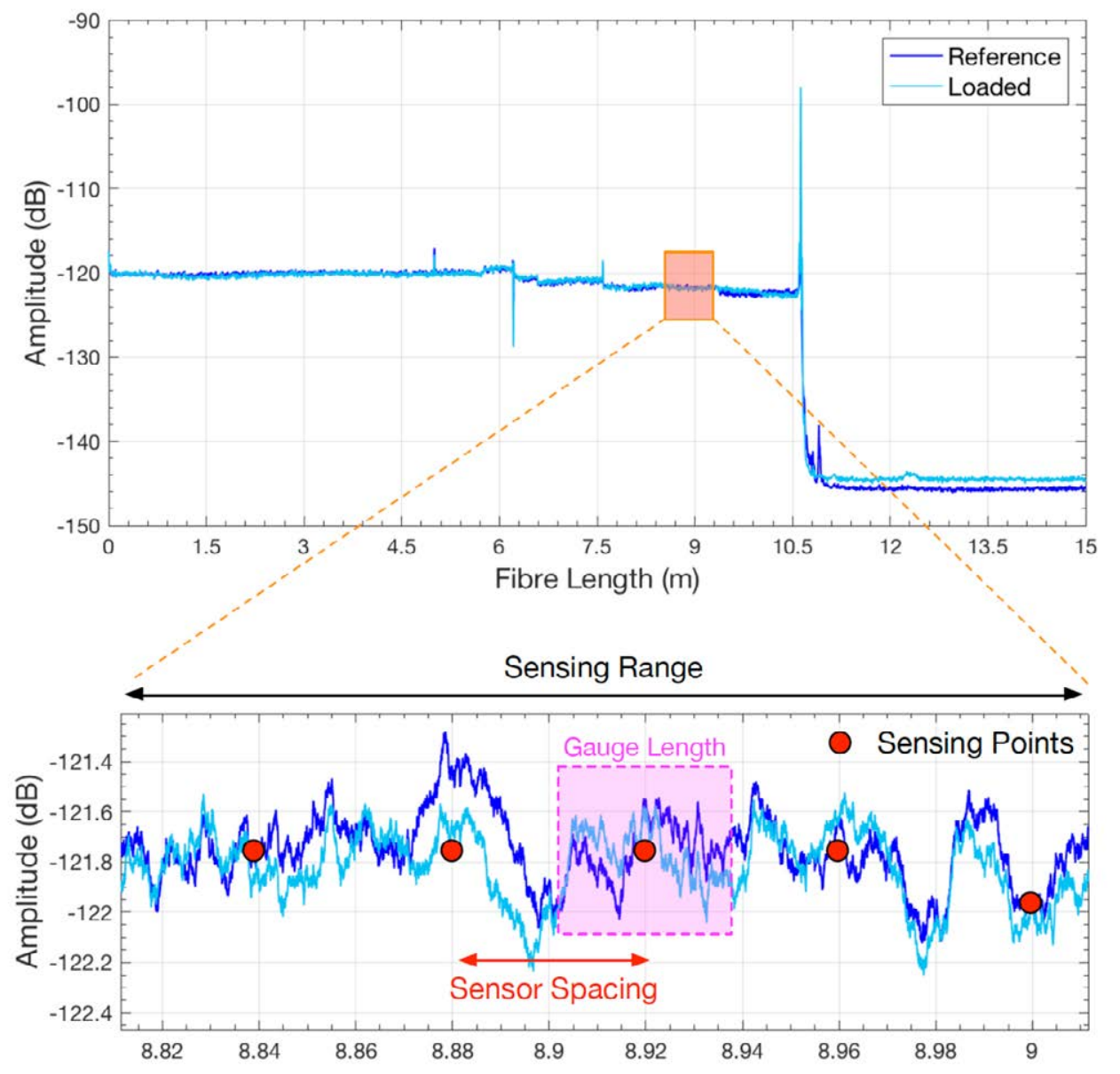

Figure 44. OBR parameters for signal conditioning. (Upper) Total reflectivity measurement for the reference and loaded measurement. (Lower) Specific signal conditioning parameters. 


\subsection{Spectral Shift Calculation}

In Figure 45, the pattern of the optical fibre is clearly shown. This is the Fourier transform of the reflection spectrum for a selected gauge length. The spectral shift, which is transformed to obtain the frequency shift $\Delta v$, can be observed in two significant peaks. The blue curve corresponds to the strained measurement, whereas the grey curve is the selected reference. The signals included in the red dashed square are shown in detail below (Figure 45b and Figure 45c). Here, in this case, the spectral shift is recognized directly. Then the Figure $45 \mathrm{~d}$ is the result of the cross-correlation performed between these two measurements. As a result, the maximum sample shift is obtained at 21 samples. However, at 20 lag of sample shift has also a significance cross-correlation value.
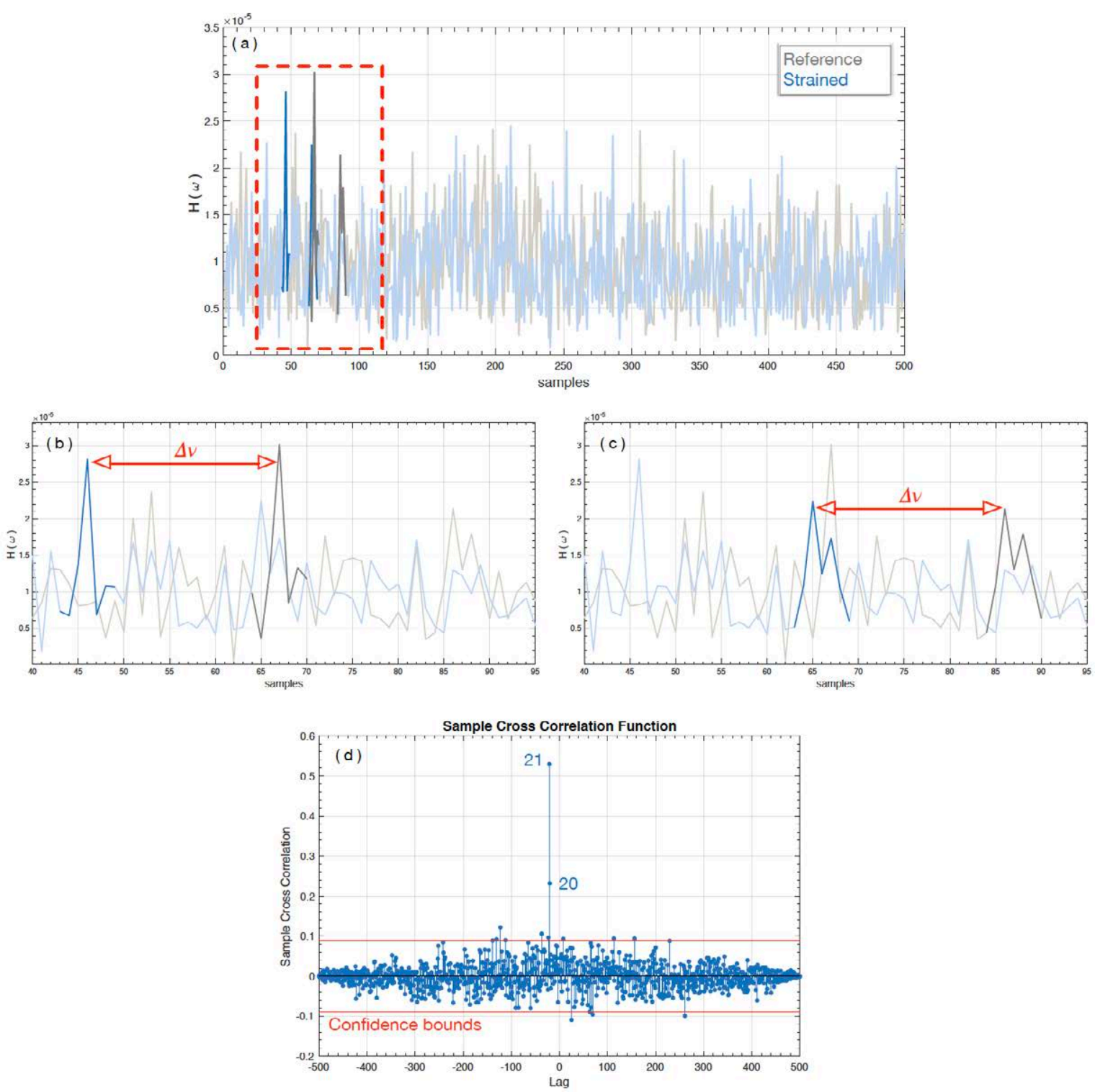

Figure 45. Spectral shift between the reference and the loaded of an OBR measurement. (a) Rayleigh Backscatter Amplitude in frequency domain. 
The correct calculation of the cross-correlation between the reference and the strained signal is one of the most important parts to obtain a precise distributed measurement (Box, Jenkins, \& Reinsel, 1994). In the algorithm developed to process the OBR measurement, the crosscovariance of the signals $y_{1 t}$ and $y_{2 t}$ is first obtained as shown in Equation 3-14 and Equation 3-15.

$$
c_{y 1 y 2}(k)= \begin{cases}\frac{1}{T} \sum_{t=1}^{T-k}\left(y_{1 t}-\overline{y_{1}}\right)\left(y_{2, t+k}-\overline{y_{2}}\right) ; k=0,1,2, \ldots, N \\ \frac{1}{T} \sum_{t=1}^{T+k}\left(y_{2 t}-\overline{y_{2}}\right)\left(y_{1, t+k}-\overline{y_{1}}\right) ; k=0,-1,-2, \ldots, N\end{cases}
$$

Where $\overline{y_{l}}$ denotes the mean of the signal and $k$ are the different lags (in general $N=300$ ). Then, considering the standard deviations of the signals are calculated as expressed in Equation 3-16 and Equation 3-17.

$$
\begin{aligned}
& s_{y 1}=\sqrt{c_{y 1 y 1}(0)}=\sqrt{\operatorname{Var}\left(y_{1}\right)} \\
& s_{y 2}=\sqrt{c_{y 2 y 2}(0)}=\sqrt{\operatorname{Var}\left(y_{2}\right)}
\end{aligned}
$$

Finally, the estimation of the cross-correlation at lag $k$ is expressed in Equation 3-18.

$$
r_{y 1 y 2}(k)=\frac{c_{y 1 y 2}(k)}{s_{y 1} s_{y 2}} ; k=0, \pm 1, \pm 2, \ldots
$$

Finally, the three main scenarios that it can be encountered when the cross-correlation is performed are shown in Figure 46. First scenario (upper figure) shows the zero value, i.e. zero lags. This occurs when both reference and loaded measurement are almost perfectly correlated and the shift is centred at zero since there is no-delay between signals. Then, the second scenario (middle figure) occurs when in the area where the optical fibre is strained or heated compared to the reference measurement. In such case, depending on the compressive, tensile, heated or cooled, the measurement is delayed or advanced (positive and negative lags respectively). Furthermore, the resultant value of the cross correlation expressed by the colour bar should be pointed out. The last scenario (lower figure) occurs when the signals are completely uncorrelated. This behaviour is encountered in cases such as, low amplitude level (lower that $-130 \mathrm{~dB}$ ), comparison between two different fibres (different reflectivity pattern) or same fibre but different gain, i.e. different number of points. 

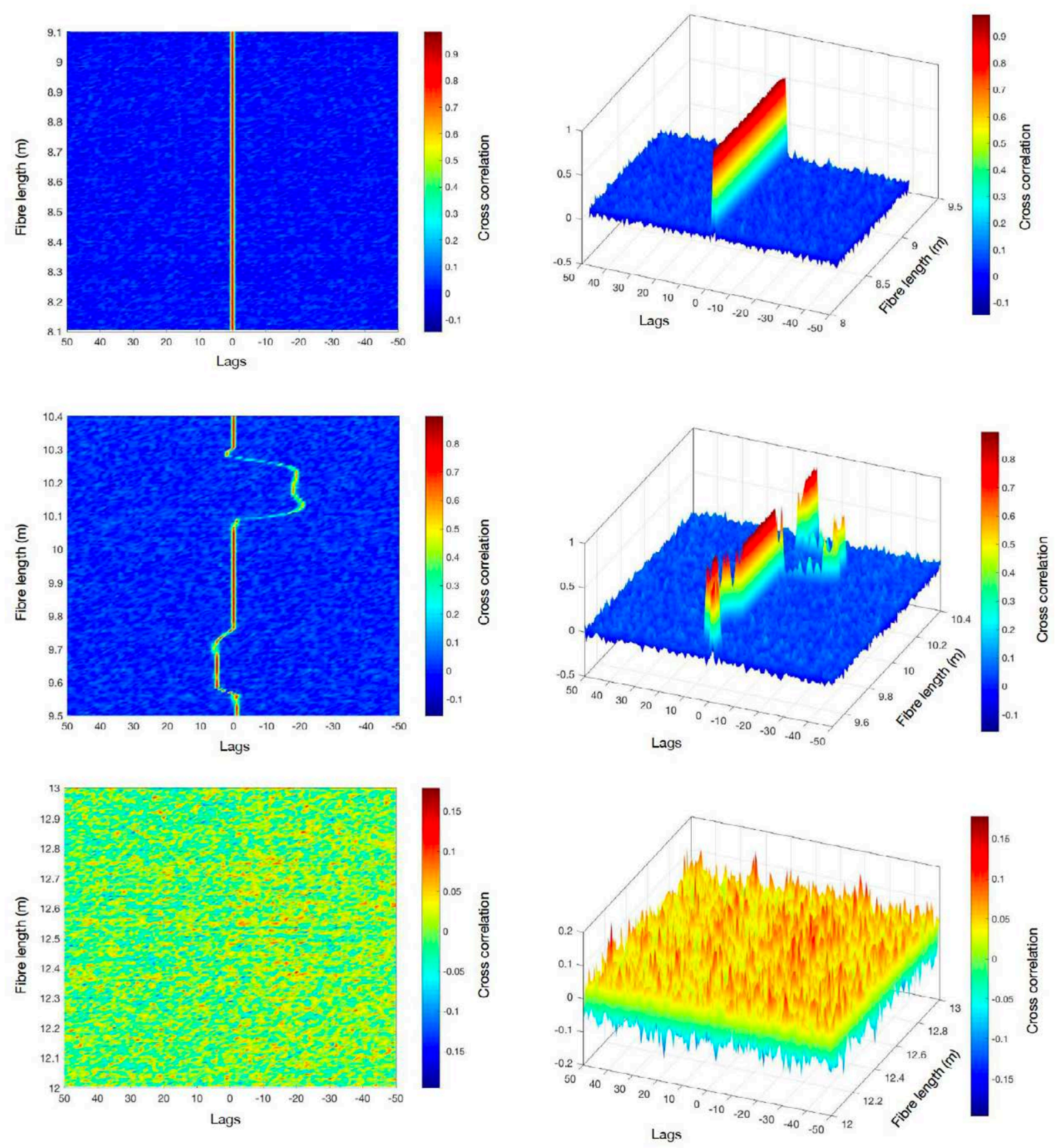

Figure 46. Results of the cross-correlation in different fibre sub-sections.

In order to consider all significant points in the cross-correlation above the confidence bounds, a cubic spline interpolation can be performed as shown in Figure 47. Then, the maximum of the cross correlation is obtained through the interpolated data. This methodology enables to obtain a more accurate calculation of the spectral shift since the influence of the residuals is also considered. This methodology was also proven to obtain long-range distributed temperature and strain measurement (Song et al., 2014). 


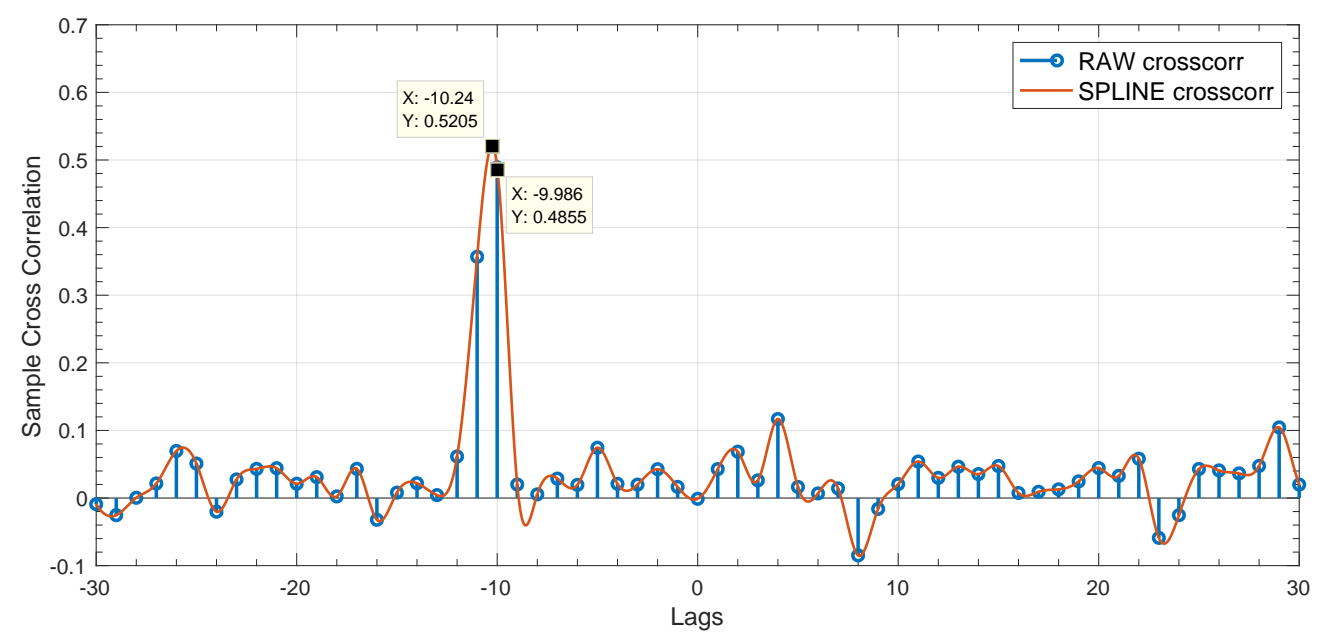

Figure 47. Raw data obtained in the cross correlation of the distributed measurement and the cubic spline interpolation of the raw data.

\subsection{Conversion in Temperature and/or Strain Changes}

Strain and temperature changes can be calculated by means of the spectral shift conversion. The conversion is carried out through a polynomial of $4^{\text {th }}$ degree which performs the relationship between both magnitudes. The temperature and strain polynomial are shown in Equation 3-19 and Equation 3-20, respectively.

$$
\begin{gathered}
\text { Temperature }=A_{0}+A_{1} x+A_{2} x^{2}+A_{3} x^{3}+A_{4} x^{4} \\
\text { Strain }=B_{0}+B_{1} x+B_{2} x^{2}+B_{3} x^{3}+B_{4} x^{4}
\end{gathered}
$$

By default, the OBR system is calibrated for a standard optical fibre single mode SMF-28 $\mathrm{e}^{+}$. For this type of fibre all coefficients have zero value except $A_{1}=0.8014$ and $B_{1}=6.668$. In the presented work, this calibration was used for all the experiments. Otherwise, if another FO type is used, OBR needs to be calibrated.

This being said, the OBR measurement cannot separate the influence of the temperature and the strain when they are acting over the DUT simultaneously by itself. Hence, the resultant spectral shift is the combination of both effects acting along the DUT. However, a temperature compensation can be performed by controlling the room temperature of the experiments. Then, the temperature effect can be easily removed considering that all fibre length is submitted to the same temperature. The main disadvantage of using this technique to compensate the temperature effect is that it considers that all fibre is under the same temperature. Thus, when a high temperature hot spot occurs all along the length of the fibre it can become undetectable. Nevertheless, the experiments presented in this thesis address 
the strain changes to detect damage in the structure and the room temperature is controlled to ensure that it remains constant. Otherwise, it would be compensated as explained hereabove.

In case of strain absence, the temperature change can be written as Equation 3-21, where $\bar{\lambda}$ is the centre wavelength of the scan range, $c$ is the light speed and $K_{T}$ is the temperature coefficient and $\Delta v$ is the frequency shifts. And, in case of no temperature changes, the conversion to strain can be calculated as Equation 3-22, where $K_{\varepsilon}$ is the strain coefficient.

$$
\begin{gathered}
\text { Temperature change }=\Delta T=-\frac{\bar{\lambda}}{c K_{T}} \Delta v \\
\text { Strain changes }=\varepsilon=-\frac{\bar{\lambda}}{c K_{\varepsilon}} \Delta v
\end{gathered}
$$




\section{Chapter 4 DAMAGE DETECTION METHODOLOGY}

This chapter focuses on presenting the methodologies applied to obtain all the results of this thesis. It highlights the importance of post-process and the measurement obtained to detect damage. Additionally, the common errors, its detection and correction are explained in detail.

\subsection{Introduction}

The whole task sequence followed for each of the experimental test used for validation is summarised in Figure 48. These tasks are presented in a general point of view and not only for distributed fibre optic sensing technique since more sensing techniques were used. In the following chapters, each of these steps are described in detail and specified for DFOS technique. The four different levels can be summarized as follows:

- Experimental test and sensor network definition: depending on the test coupon (for local approach) or structure (for global approach) a different sensing network should be selected. This network must be suitable for each purpose of damage monitoring.

- Data acquisition (DAQ): in order to obtain the read-out of the sensing network, a suitable DAQ system should be provided.

- Data pre-processing: the data, prior to be used for the analysis functions, has to be pre-processed for signal acquisition error detection and correction as well as signal conditioning.

- Data analysis: this task includes the analysis of the pre-processed data by means of the developed algorithms for SHM purposes. 


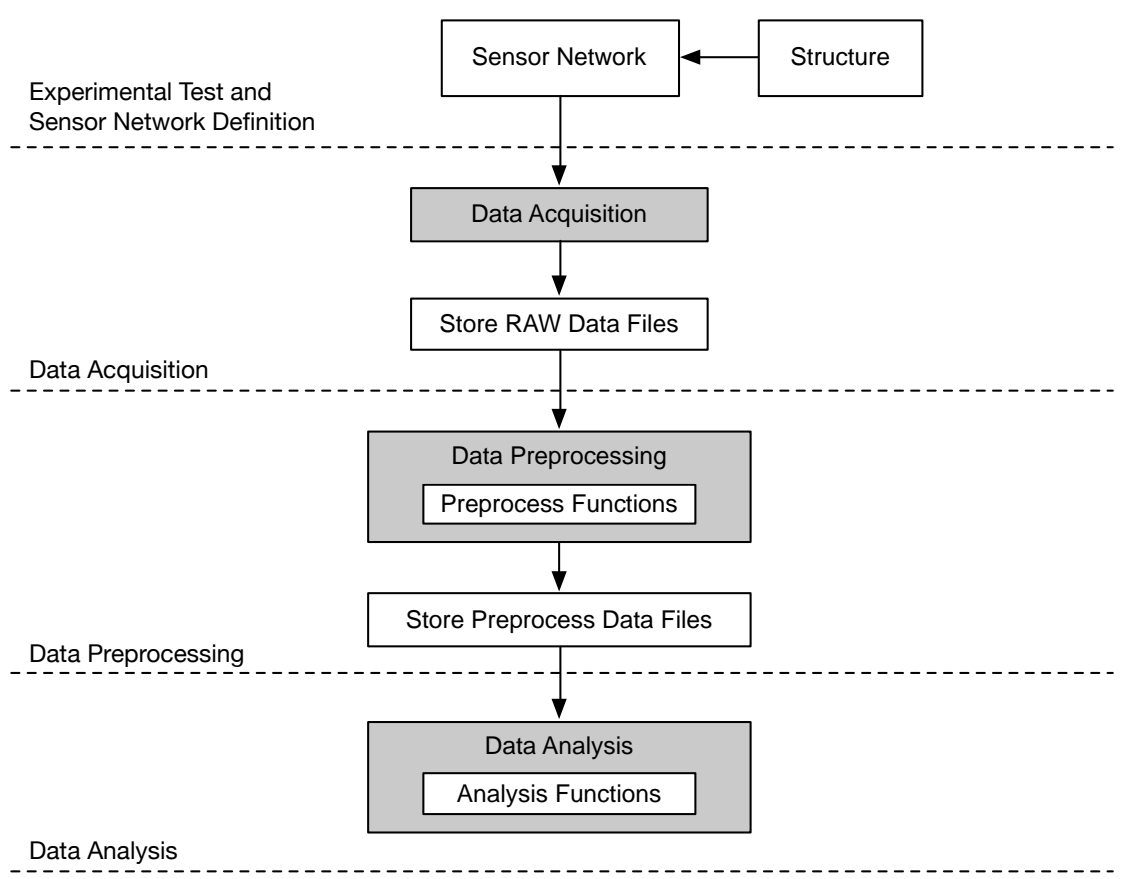

Figure 48. Tasks sequence for each experimental test.

\subsection{Experimental Tests and Sensor Network Definition}

The experimental validation is carried out in two different and contrasting approaches:

- In the first place, local damage validation in test coupons takes place at the laboratory level in order to explore different techniques to detect damage in a more controlled and cleaner fashion. The sensing network defined for these experiments was straightforward and limited to DFOS technique.

- In second place, global damage validation for a real aircraft structure in a full and complex experimental test. Despite the DFOS technique, different sensing techniques are also used to compare the reliability of each technique and the ability to detect and locate damage. In this case, utilizing the sensing network combined, not only fibre optics for distributed measurement, but also thermocouples for temperature monitoring, strain gauges and rosettes and a highspeed camera.

The detail of the different experimental test used for validation are summarized in Table 4 . 
Table 4. Experimental test summary

\begin{tabular}{|c|c|c|c|c|}
\hline Approach & $\begin{array}{c}\text { Experimental } \\
\text { Test }\end{array}$ & Damage Monitoring & Sensing Network & Dest \\
\hline Local & $\begin{array}{c}\text { Double-lap } \\
\text { joint }\end{array}$ & $\begin{array}{c}\text { Flaw detection and } \\
\text { location in the } \\
\text { overlapping area }\end{array}$ & Optical fibre & 5.1 \\
\hline \multirow{2}{*}{ Global } & $\begin{array}{c}\text { Free-edge } \\
\text { delamination }\end{array}$ & $\begin{array}{c}\text { Delamination damage } \\
\text { detection and location }\end{array}$ & Optical fibre & 5.2 \\
\hline & Stiffened & $\begin{array}{c}\text { Buckling, fatigue and } \\
\text { damage growth }\end{array}$ & $\begin{array}{c}\text { Optical fibre, rosettes } \\
\text { strain gauges, } \\
\text { thermocouple, high } \\
\text { speed-camera }\end{array}$ & 6.1 \\
\hline
\end{tabular}

\subsection{Data Acquisition}

Figure 49 presents all sensor networks used with their corresponding DAQ system and file type in their data storage folder.

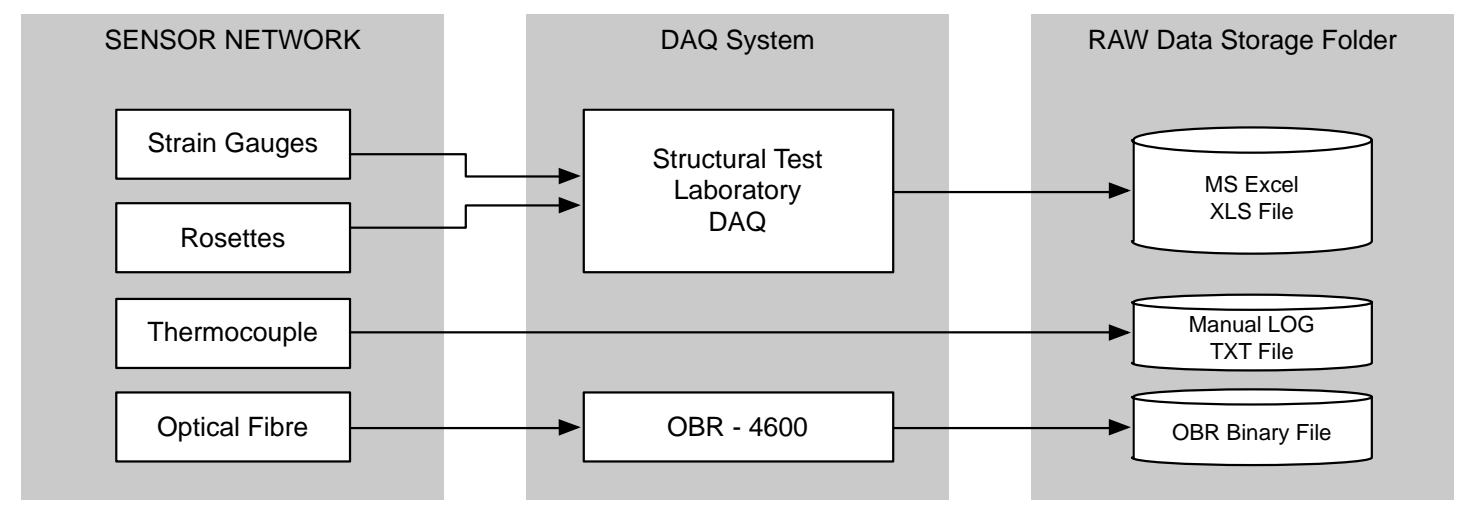

Figure 49. Data acquisition for different sensor network.

All OBR measurements presented in the validation results were recorded under static loads and stored in a file with an extension of *.obr. These are binary files that can be opened and managed by Luna Technologies software.

\subsection{Data Pre-processing}

All measurements acquired by the different sensors were pre-processed prior to be used for damage monitoring.

In the case of distributed measurements, the pre-processing is carried out in two steps: 
- First, the RAW data acquired during the structural test is called from the OBR software. Then, the signal conditioning parameters (3.7) and the measurable parameter, e.g. strain and/or temperature, has to be chosen to generate the *.txt file based on the selection. Finally, the new *.txt file is stored which includes the information of the measurable parameter against length and can be used now for error detection and correction. The aspect of this file is shown in Figure 50.

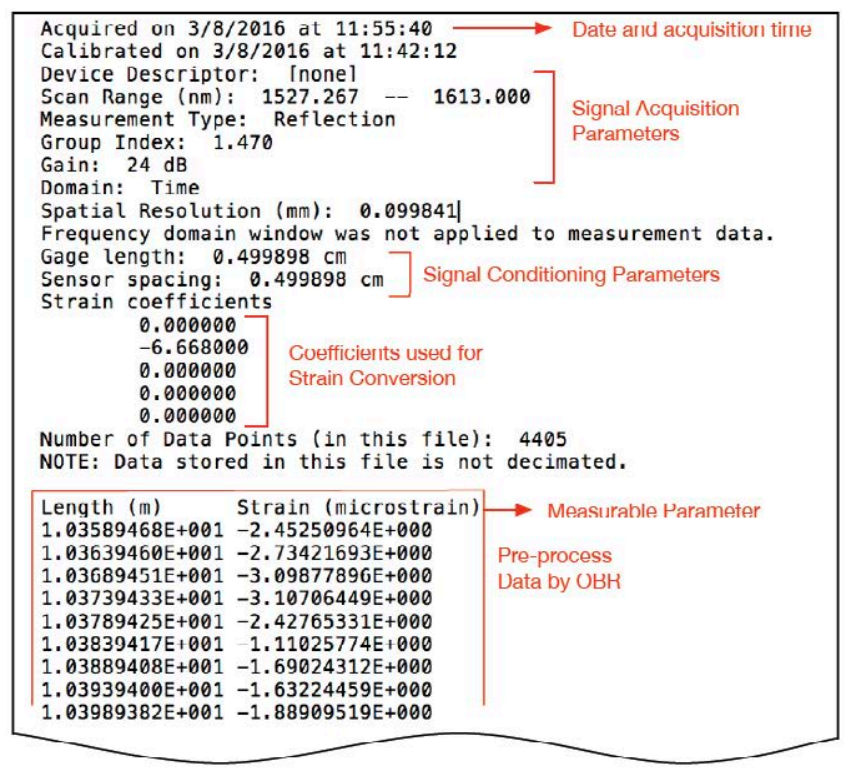

Figure 50. Output file of the OBR system after signal conditioning parameters selection.

- Second, once *.txt is generated, it is read and converted into *.mat files through the algorithms developed for this purpose in a Matlab ${ }^{\circledR}$ environment. Then, the signal is filtered in order to detect and correct possible errors. These two steps are summarized in the following Figure 51.

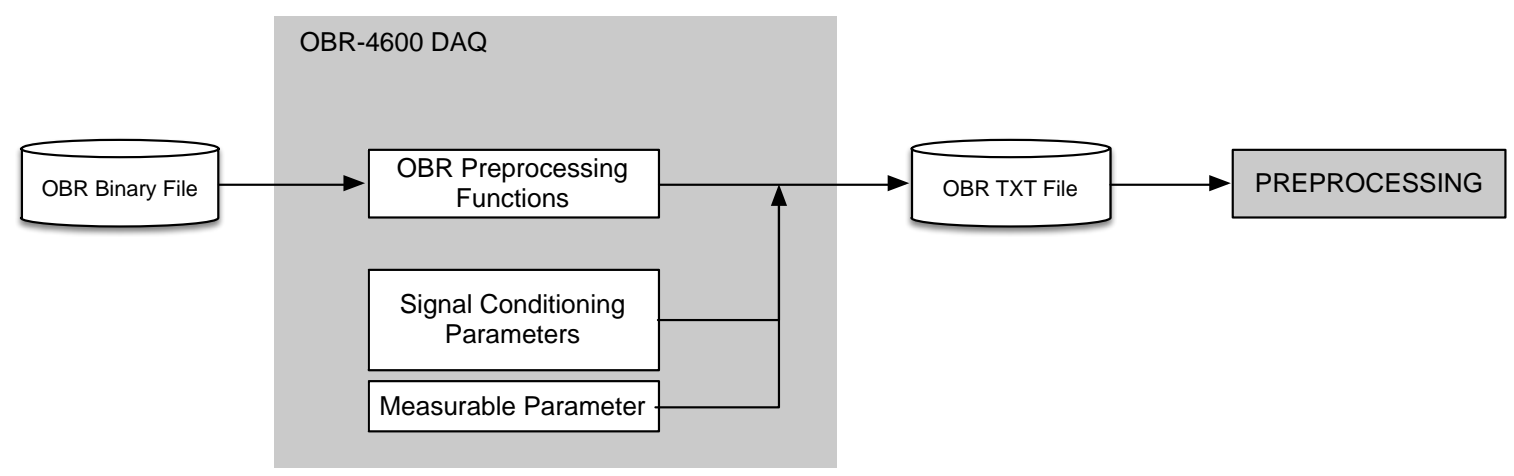

Figure 51. OBR pre-processing flowchart.

The following section thoroughly presents the error detection and correction (EDC) for distributed measurement. 


\subsubsection{Error Detection and Correction for Distributed Measurement}

First, prior to using the data for damage analysis, it should be filtered to detect possible errors, such as spikes and noise. Then, once these errors are well identified in the measurement, they can be corrected. The main classification of the issues encountered in the OBR distributed measurement fall into two categories depending on whether the source is the signal acquisition, or if the source lies in the signal pre-processing in the OBR software. On one hand, the signal acquisition issues are unable to measure, and they have to be solved before the experiment is performed. On the other hand, signal pre-processing errors may occur due to a wrong selection of signal conditioning parameters.

(a)

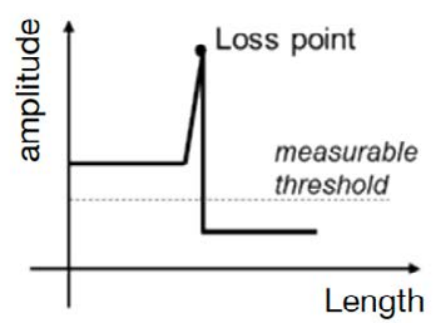

(c)

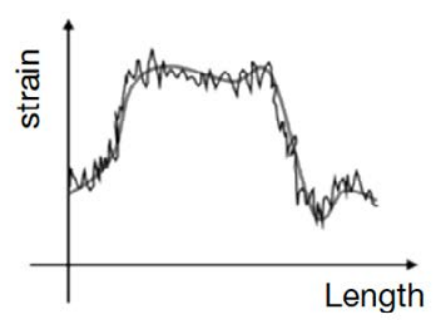

(b)

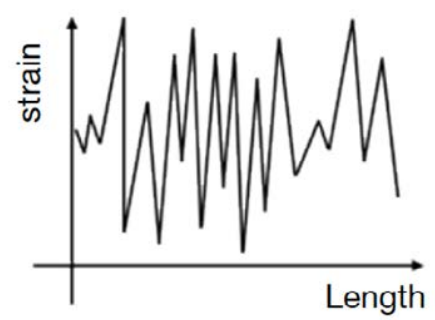

(d)

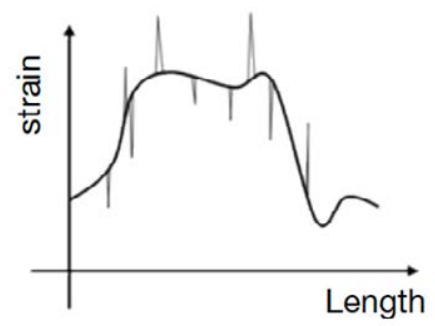

Figure 52. (a) Optical losses, (b) global signal noise, (c) local signal noise and (d) signal spikes.

Figure 52 depicts the most common issues. These errors can occur under different conditions and most of them can be solved if they are detected in an early state. The Table 5 is an attempt to describe these errors and issues in detail, as well as, the possible corrections that can be carried out. 
Table 5. Common errors and issues for OBR distributed measurements.

\begin{tabular}{|c|c|c|c|}
\hline Source & Issue & Possible Cause & Possible Corrective Action \\
\hline \multirow{6}{*}{$\begin{array}{l}\text { Signal } \\
\text { acquisition } \\
\text { issues }\end{array}$} & \multirow{5}{*}{ Optical losses } & $\begin{array}{c}\text { Minimum loop radius } \\
\text { exceeded }\end{array}$ & Position readjustment \\
\hline & & Ingress/ Egress bending & Position readjustment \\
\hline & & Dirt on connector faces & Cleaning procedures \\
\hline & & Fibre breakage & $\begin{array}{l}\text { Fibre replacement or splice } \\
\text { (if possible) }\end{array}$ \\
\hline & & Fabry-Pérot & $\begin{array}{l}\text { Fibre replacement or splice } \\
\text { (if possible) }\end{array}$ \\
\hline & Global signal noise & $\begin{array}{l}\text { Unalike acquisition } \\
\text { parameters }\end{array}$ & Retake measurement \\
\hline \multirow{3}{*}{$\begin{array}{l}\text { Signal pre- } \\
\text { processing } \\
\text { errors }\end{array}$} & \multirow{2}{*}{ Signal spikes } & Wrong signal conditioning & GL and SS readjustment \\
\hline & & Transversal shear stress & $\mathrm{n} / \mathrm{a}$ \\
\hline & Local signal noise & Wrong signal conditioning & $\begin{array}{l}\text { GL and SS readjustment or } \\
\text { smoothing }\end{array}$ \\
\hline
\end{tabular}

\subsubsection{Distributed Signal Measurement Smoothing}

The local signal noise of the distributed measurement is one of the most typical behaviour found in these signals. As explained above, the source of it can be caused due to a wrong signal conditioning. But also, this behaviour may appear with small strain level or for no apparent reason. This aspect gains importance when the derivative of these signals is intended to be obtained in data analysis for damage monitoring. Therefore, the application of a filter to reduce the signal noise becomes even more necessary.

As explained in 3.2, distributed measurements can be considered as a discrete signal which provides single strain values. These values are calculated by the cross-correlation in a gauge length selected from a sensing range of the optical fibre. Consequently, the derivative of these signals is likely to become noisy. Therefore, prior to derivate a distributed strain measurement, is extremely important to filter the signals to obtain reliable results. For such reason, after using an adequate filter, the signals become smoother, and they can be differentiated without any regard for the appearance of noise. Overall, the greater the level of local distributed measurement noise, the more important the application of a signal filter becomes. In these measurements, a Savitzky-Golay smoothing filter (Savitzky \& Golay, 1964) was applied to the signals acquired by the OBR.

$$
f_{k}(x)=\sum_{i=0}^{k} c_{i} x^{i}
$$


As discussed in Patricia F. Díaz-Maroto et al., (2018), the full-field strain measurement, is fitted by means of a polynomial based on the least square method where the selection of the polynomial order must be selected as an input. Unlike other filters, the polynomial fitting (Equation 4-1) is applied at consecutive frames, which allows to calculate the coefficients $c_{i}$ of the polynomial in more accurate way. In addition, it should be noted that the usage of the presented filter enables the statistical moments to preserve. In this case, all distributed measurements were fitted with a cubic polynomial degree $(k=3)$ and considering 7 samples of frame. The selection of these parameters was carried out according to Figure 53, which demonstrates to be a good approach in order to support inflections and signal smoothing. As can be seen in this figure, the greater the polynomial degree chosen, the lesser smoothness is achieved, whereas the greater the frame selected, the more curve smoothness is produced. Finally, the parameters choice was made based on the maximum RMSE under $1 \mu \varepsilon$ (OBR strain resolution) with the lower computational cost, i.e. the lower polynomial degree to fit the polynomial.
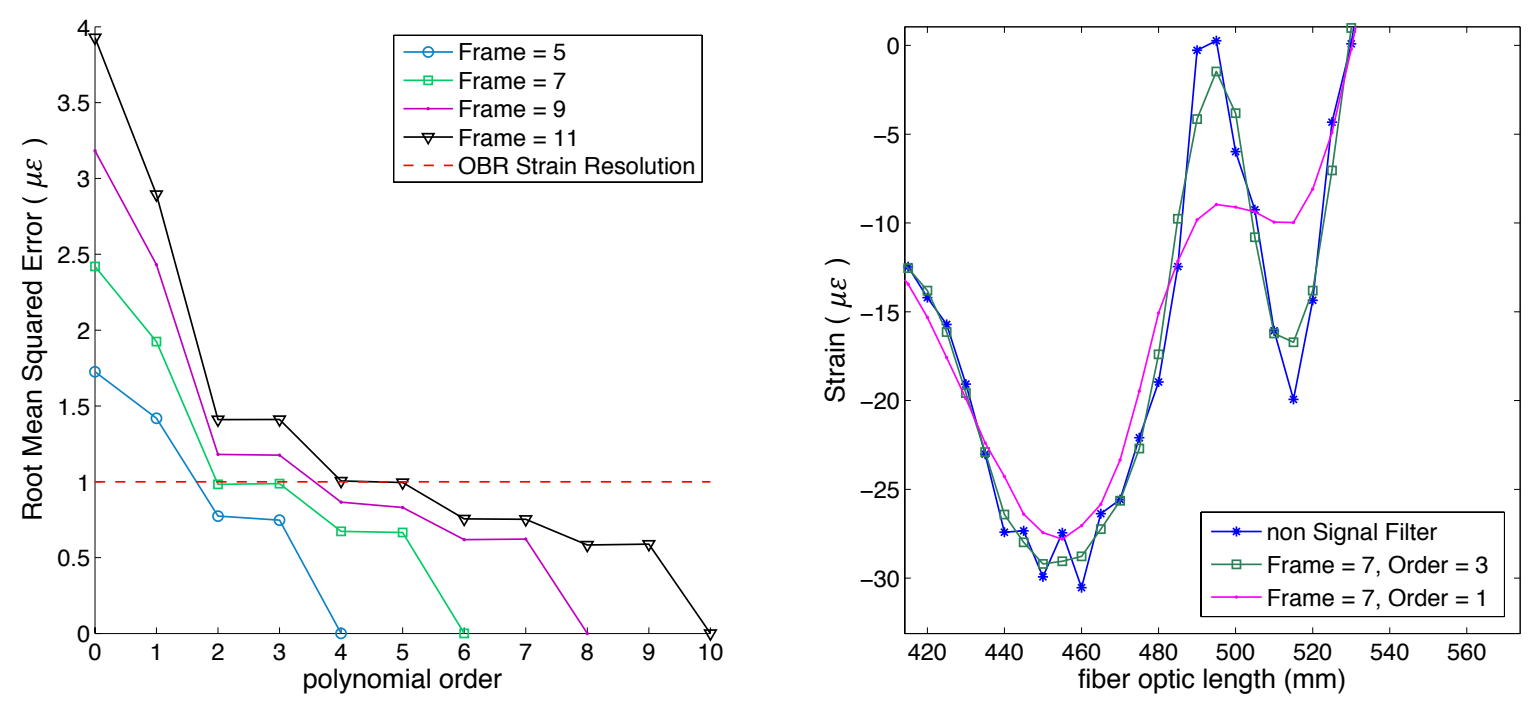

Figure 53. Polynomial order of Savitzky-Golay filter and RMSE in distributed strain approximation (Patricia F. Díaz-Maroto et al., 2018).

It should be highlighted the influence of the filter application. In Figure 54 (left) the first derivative of a set of distributed strain measurements with no-filter is displayed. The derivative signals tended to be noisy since original curves were not smooth. Then, in Figure 54 (right), a Sgolay filter is applied to each measurement before the calculation of the derivative. As a result, the distributed measurements can be now distinguished and the tendency for each of them is demonstrated. The edge should also be taken into account since the first frame of the Sgolay filter can induce worthless values which is the so-called edge effect. Thus, these sensing points should not be taken into account as part of the measurement. 

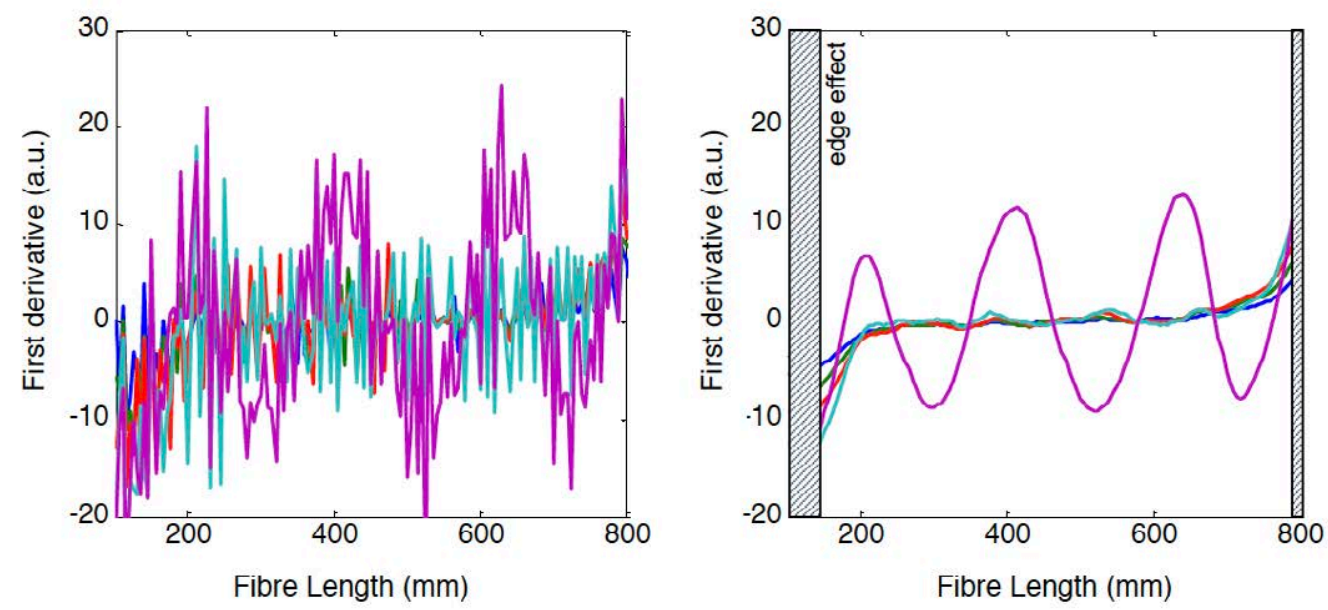

Figure 54. (Left) First derivative of initial strain measurements. (Right) First derivative after Sgolay filter applied

\subsection{Data Analysis}

The tasks for data analysis of the different experimental tests involves the same structure:

- Damage detection: this step lies in the analysis of the pre-processed data to identify any damage in the structure under research. In the different experimental tests, the methodologies used are:

- Residual strains.

- Load-Strain scattering.

- Second derivative analysis.

- Damage location: the relationship between the sensor network and the structure should be known previously in order to locate the damage event. Regarding the optical fibre, a layout should be performed so as to transform each original distributed measurement into the local positioning

Original Positioning

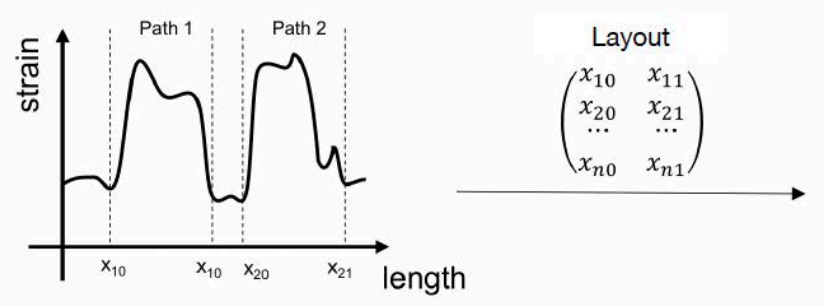

$\varepsilon=f\left(X_{F O}\right)$
Local Positioning

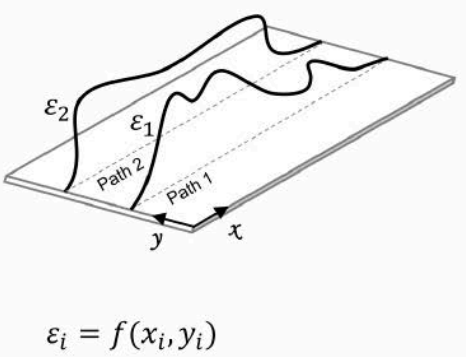

Figure 55. Relationship between the original sensing location in the fibre optic length and the local positioning on the structure through the layout matrix. 
- Damage characterization: in this step the evaluation of the damage is performed. Also, depending on the structural test the characterization of the damage will be further discussed.

\subsubsection{Damage Detection by Load-Strain Scattering}

In those experimental tests in which the structure is loaded, it can be used to represent the strain-load curves and analyse the loss of the linearity.

This methodology has been commonly used to detect non-linear appearance from the loadstrain measurement of the strain gauges and it was the so-called Bifurcation method (Hoff N.J., Boley B.A., 1948). At the moment of non-linear onset, the surface of the structure is sharply deformed, and the linear behaviour of the load-strain curve is lost.

This methodology can be also extrapolated for distributed measurements since they can be considered discrete sensing points to obtain several load-strain curves to reproduce the readout of a strain gauge. Then, it is straightforward to detect the load step where non-linear commences. This methodology has been successfully proven in three stiffened composite panels by means of distributed strain measurement (Patricia; F.Díaz-Maroto et al., 2015).

Next Figure 56 shows an example of the application of this methodology on a composite reinforced panel. Random points denoted by $x_{i}$ were selected over the length of the distributed strain measurement and load-strain curves plotted for these sensing points. The initiation of non-linear behaviour started at a load of $550 \mathrm{kN}$ where the curves were scattered apart from one another.
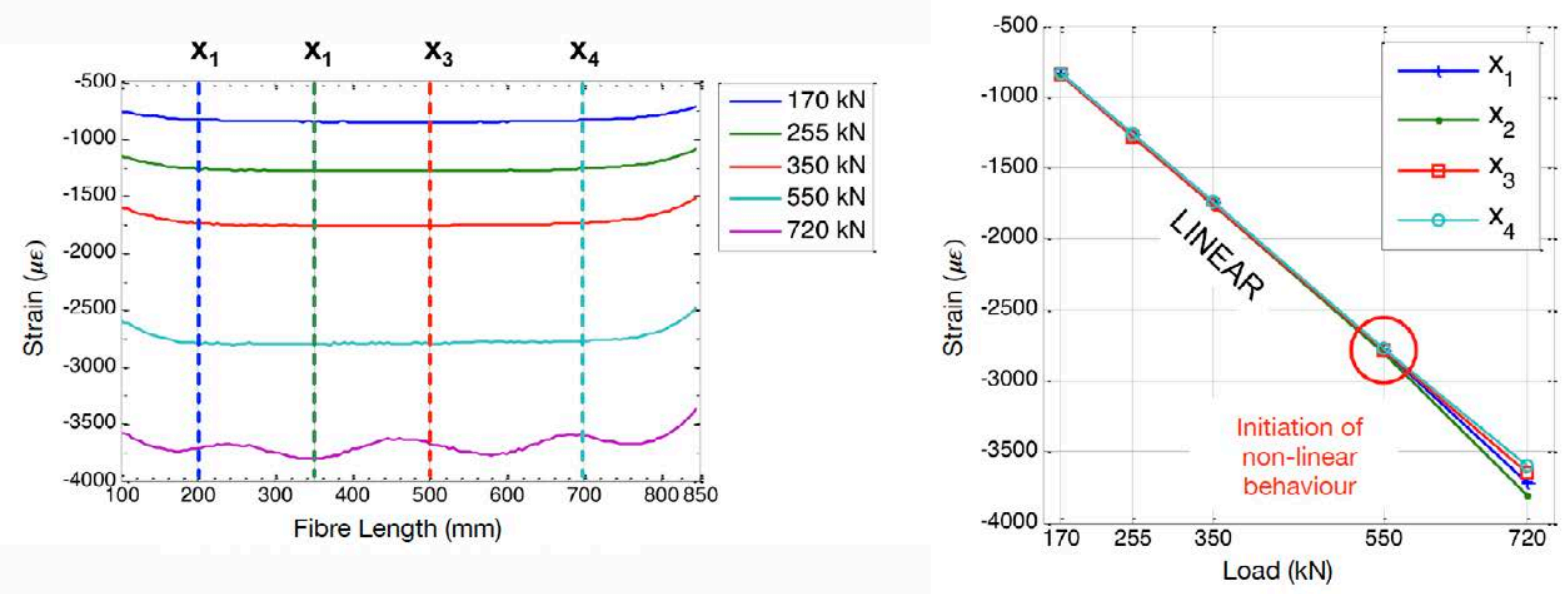

Figure 56. (Left) Distributed strain measurements on a composite reinforced panel. (Right) Strain against load for random sensing points (Patricia; F.Díaz-Maroto et al., 2015). 


\subsubsection{Damage Detection by Second Derivative Analysis}

The mode-shape based structural damage is presented as an emerging research field for damage identification. Many authors have already demonstrated that vibrational mode shapes can be used as an indicator of damage in different structures ((Gentile \& Messina, 2003; Jiang, Ma, \& Ren, 2012)). These authors address the detection of damage to curvature mode shape sensibility.

The term "curvature mode shape" was already introduced to use the difference in the curvature mode shape to detect and locate damage (Pandey \& Biswas, 1994; Pandey, Biswas, \& Samman, 1991). Therefore, when a structure is damaged, it undergoes changes in the rigidity (EI) which can be identified directly from the changes in curvature $v^{\prime \prime}(x)$. In a specific location $x$, the curvature $v^{\prime \prime}(x)$, can be obtained in general terms with the differential equation of the elastic curve as shown in Equation 4-2.

$$
v^{\prime \prime}(x) \approx \frac{M(x)}{E I}
$$

where $M(x)$ is the moment at a specific location $x$ and changes in curvature were obtained numerically by using a central differential approximation defined as in Equation 4-3.

$$
v_{i}^{\prime \prime}=\frac{v_{i+1}-2 v_{i}+v_{i-1}}{h^{2}}
$$

More recently, Roy (2017) formulates the derivatives of the mode shapes corresponding to damage and undamaged states of the structure. The difference in mode shape slope is presented as a Dirac delta function to detect and locate the presence of damage. Then, the derivative of the measurement allows to amplify the discontinuities in the mode shape as a consequence of local damage.

The susceptibility to noise of second order derivative mode shapes has been also been explored to provide a reliable damage identification (Cao, Radzieński, Xu, \& Ostachowicz, 2014).

In this thesis, the first derivative of the distributed measurements is used to characterise the shape of the fibre optic path when it is submitted to different loads. Hence, linear behaviour is identified since no significant changes in the first derivative is appreciated. So, the second derivative applied to each sensing point with respect to the load allows to identify changes from one step to another.

This methodology was developed to identify the buckling appearance in a composite reinforced panel (Chapter 6). However, this technique can also be extrapolated to detect any non-linear behaviour by means of a dense distributed sensing network. 
As explained in Patricia F. Díaz-Maroto et al., (2018), firstly, each of the distributed measurement must be filtered (4.4.2) in order to obtain its first order derivative. Afterwards, the second derivative can be obtained to measure the discrepancy in the shape of strain field for each load step. This second derivative measurement provides a local approach of how each of the sensing points that shape the measurement is being loaded since the derivative magnitude only depends on the slope. Consequently, if the second derivative level is low, it will indicate an expected behaviour of the structure when is loaded (no slope changes). However, high values in the second derivative will correspond to a non-linear strain response against load. In this case, potential failure points should be considered in these locations.

The distributed strain measurement is considered as a vector of $\mathrm{N}$ sensing points for each load step. For this reason, the calculation for the m-load step $l_{m}$ is calculated as shown in Equation 4-5.

$$
\begin{gathered}
\varepsilon\left(x, l_{m}\right)=\left[\varepsilon\left(x_{1}, l_{m}\right), \quad \varepsilon\left(x_{2}, l_{m}\right), \ldots \quad \varepsilon\left(x_{N}, l_{m}\right)\right] \\
\varepsilon\left(x, l_{1}\right)=\left[\begin{array}{lll}
\varepsilon_{1,1}, & \varepsilon_{2,1}, \ldots & \varepsilon_{N, 1}
\end{array}\right]
\end{gathered}
$$

As a result, after using a Savitzy-Golay filter of third degree, first strain derivative can be obtained as expressed in the Equation 4-8.

$$
\begin{aligned}
& \tilde{\varepsilon}\left(x, l_{m}\right)=\left[\sum_{i=0}^{3} c_{1 i} x^{i}, \sum_{i=0}^{3} c_{2 i} x^{i}, \ldots \sum_{i=0}^{3} c_{N i} x^{i}\right]=\left[\begin{array}{lll}
\tilde{\varepsilon}_{1, m}, & \tilde{\varepsilon}_{2, m}, \ldots & \tilde{\varepsilon}_{N, m}
\end{array}\right] \quad 4-6 \\
& \tilde{\varepsilon}_{x}\left(x, l_{m}\right)=\left[\begin{array}{lll}
\tilde{\varepsilon}_{2, m}-\tilde{\varepsilon}_{1, m}, & \tilde{\varepsilon}_{3, m}-\tilde{\varepsilon}_{2, m}, \ldots & \tilde{\varepsilon}_{N, m}-\tilde{\varepsilon}_{N-1, m}
\end{array}\right] \\
& \tilde{\varepsilon}_{x}\left(x, l_{1}\right)=\left[\begin{array}{lll}
\tilde{\varepsilon}_{x_{1, m}}, & \tilde{\varepsilon}_{x_{2, m}}, \ldots & \tilde{\varepsilon}_{x_{N-1, m}}
\end{array}\right]
\end{aligned}
$$

Again, the filter is applied to the first derivative prior to obtain the second derivative with regard to the load in order to figure out the changes in the distributed strain shape.

$$
\begin{gathered}
\tilde{\tilde{\varepsilon}}_{x l}\left(x, l_{m}\right)=\left[\begin{array}{c}
\tilde{\tilde{\varepsilon}}_{1, m}-\tilde{\tilde{\varepsilon}}_{1, m-1}, \quad \tilde{\tilde{\varepsilon}}_{2, m}-\tilde{\tilde{\varepsilon}}_{2, m-1}, \ldots \tilde{\varepsilon}_{N, m}-\tilde{\varepsilon}_{N-1, m-1}
\end{array}\right] \\
\tilde{\tilde{\varepsilon}}_{x l}\left(x, l_{m}\right)=\left[\begin{array}{lll}
\tilde{\tilde{\varepsilon}}_{x l_{1, m}}, \tilde{\tilde{\varepsilon}}_{x l_{2, m}}, \ldots & \tilde{\varepsilon}_{x_{N-1, m}}
\end{array}\right]
\end{gathered}
$$

Finally, the universal threshold is used as a damage index to evaluate the sensing points over this limit to be considered as an abnormal behaviour (Donoho \& Johnstone, 1994). It can be defined as in Equation 4-11. 


$$
\lambda_{U}=\sigma \sqrt{2 \log n}
$$

Where $\sigma$ is the standard deviation and $n$ is the number of data points.

\subsubsection{Example of Second Derivative Analysis}

In order to show the reliability of this methodology the following example is presented in Figure 57. In this case, 6 distributed strain measurements were recorded for $-170 \mathrm{kN},-255$ $\mathrm{kN},-350 \mathrm{kN},-550 \mathrm{kN}$ and $-720 \mathrm{kN}$ of pure compressive load (Patricia; F.Díaz-Maroto et al., 2015). In the first derivative measurement, it can be noted the presence of non-linear behaviour for the last measurement. Furthermore, the second derivative signals were squared so as to amplify the non-linear event appearance acting as an index when the structure buckles.
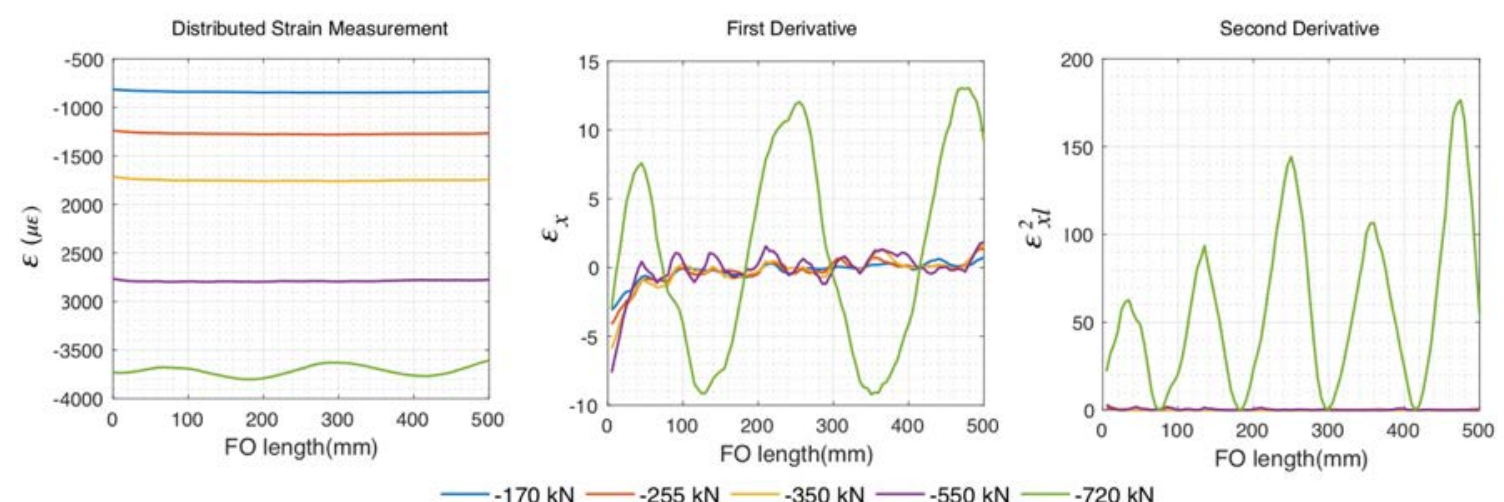

Figure 57. Example of global buckling detection by the second strain derivative analysis (Patricia F. Díaz-Maroto et al., 2018).

\subsubsection{Data Analysis Visualization}

In order to enhance the experimental results, different visualization algorithms were developed. The development of distributed measurement maps as an important technique to overview the current state of the whole structure must be noted.

\subsubsection{Distributed Measurement Maps}

Hereafter, the results from distributed measurements are often represented in distributed measurement maps, as long as the layout of the FOS network allows it. This is when the distributed sensing network is disposed covering large areas of the structure. The distributed measurement map is an algorithm developed in a Matlab® environment. 
The distributed measurement map improves the visualization related to obtain an overview of the full-field strain or temperature, i.e. the measurand parameter, of the structure. This technique allows the number of FO lines to reduce since intermediate sensing points can be predicted by linear interpolation of neighbour sensors at the surrounding area. However, the use of this technique is limited by the distance between paths, seeing as when damages occurs far from the optical fibre it can become undetectable and it will be linear interpolated.

Next Figure 58 is a diagram of how the strain map is obtained from OBR distributed measurement by the developed algorithm.

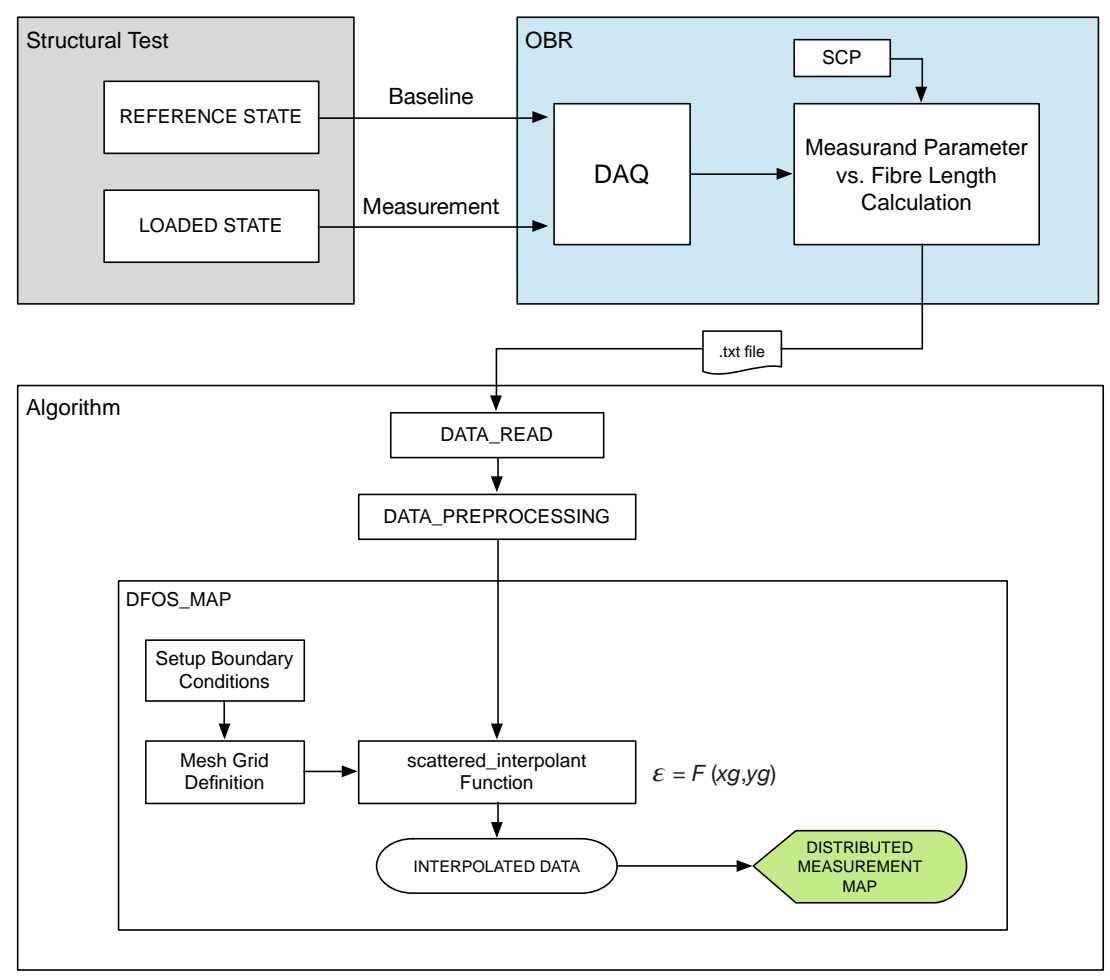

Figure 58. Flow char of the algorithm methodology to perform distributed strain maps.

This algorithm is mainly separated in three main blocks. Firstly, the OBR measurements are acquired in a binary format during the specific structural test. So as to perform a distributed strain measurement, two signals are compared, one needs to act as a baseline. Then, full-field strain or temperature is obtained in time domain and represented as a function of the optical length. Prior to perform the experimental test, it is necessary to locate the position of the optical sensing network regarding structural coordinates. The local position will be used to build a surface strain. Finally, a mesh is generated based on the $n$ selected number of sensing points and the boundary conditions, i.e., the dimensions of the area occupied by the sensing network. The strain value at the points which make up the mesh is obtained by linear interpolation. The smoother the mesh, the better spatial resolution is obtained despite the high computational cost. The heart of the matter is to figure out the most adequate mesh to obtain a good strain field approximation without overfitting. 


\section{Chapter 5 LOCAL DAMAGE DETECTION APPROACH}

In this chapter different experimental tests are presented. They consist, mostly, of laboratory tests which allow to validate the proposed methodologies for distributed measurement analysis. Therefore, the aim of this section is to detect different local damages in composite material coupons.

First, the local evaluation of a double lap joint is discussed, which includes a finite element method to establish a comparison. Second, a set of experimental delamination damage as a consequence of lowenergy impacts in free-edges is presented and studied from the residual strains.

\subsection{Local Evaluation of double lap joint.}

This experiment is focused on the local strain field around the defect of a double lap joint. The selected coupon was made in aluminium and the optical fibre was embedded into the adhesive layer. In order to compare the distributed strain measurement all along the length of the coupon, a FEM model was developed in MSC Patran software. Some of the ideas and results shown herein have appeared in the conference paper "Monitorización de uniones adhesivas con sensores distribuidos de fibra óptica" (F.Díaz-maroto, López, \& Güemes, 2015).

\subsubsection{Introduction and Experimental Motivation}

The use of adhesives has been growing in recent decades, mainly in the aeronautic field. This inclination has been fuelled by the need to reduce the structural weight. Typically, the most common use to join aeronautical structures is through rivets, which involves a high number of elements with the additional weight increment. Additionally, the stress distribution around the rivets is not uniform, which induces undesirable residual stresses. This is why the use of adhesive gives an advantage since it allows the joint to distribute the efforts uniformly along the overlap. Furthermore, the thickness of the adhesive is too small, therefore, eliminating the possibility of adding extra weight to the join.

However, there is an important issue that should be taken into account regarding the quality of the adhesive layer. It is crucial to ensure its quality during the whole life service of the structure, considering that if under harsh environments the joint can be weakened. Thus, in 
order to extend the use of the adhesive for aeronautical purposes, the ability to monitor the adhesive joint is an important challenge to overcome.

In the quest to achieve a structural health monitoring of the adhesive joint, distributed optical sensors have been presented as a good choice due to the capability of them being embedded into the adhesive. This way, the overlap area can be monitored through the distributed strain measurement.

\subsubsection{Experimental Setup}

The laboratory specimen used to monitor the full-field strain at the overlap area is shown in Figure 59. As can be seen here, the optical fibre line was embedded into the overlap at the upper face. This configuration was chosen in view of the fact that during the tensile test of the specimen, it avoids the eccentricity effect of the load at the overlap area (da Silva, Öchsner, \& Adams, 2011). Then the strain field recorded is only due to the tensile load $F$.
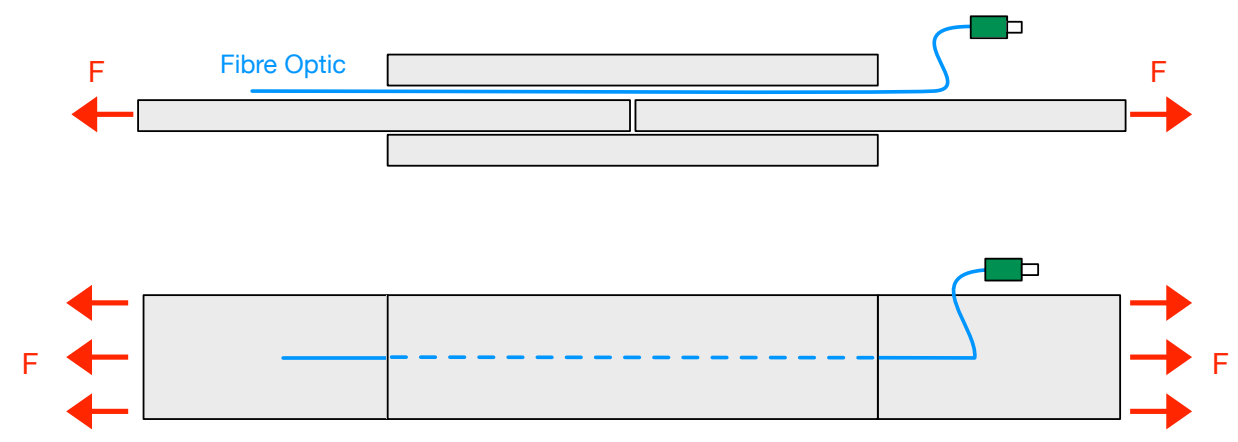

Figure 59. Double lap metallic joint monitored by two optical fibre.

The specimen was made of aluminium alloy 2024T4 by four plates with dimensions of $25 \mathrm{x}$ $115 \mathrm{~mm}$ and a thickness of $2 \mathrm{~mm}$. The coating of the selected optical fibre for this experiment was polyimide due to its high thermal resistance and smaller diameter, $150 \mu \mathrm{m}$. This makes it easier to be embeddable into the adhesive layer since it is more sensible to strain gradients. The optical fibre was located between the adhesive and the upper aluminium plate, see Figure 60. It should be noted that in order to avoid the effect of the border at ingress-egress of the optical fibre, additional adhesive was placed at those areas. 

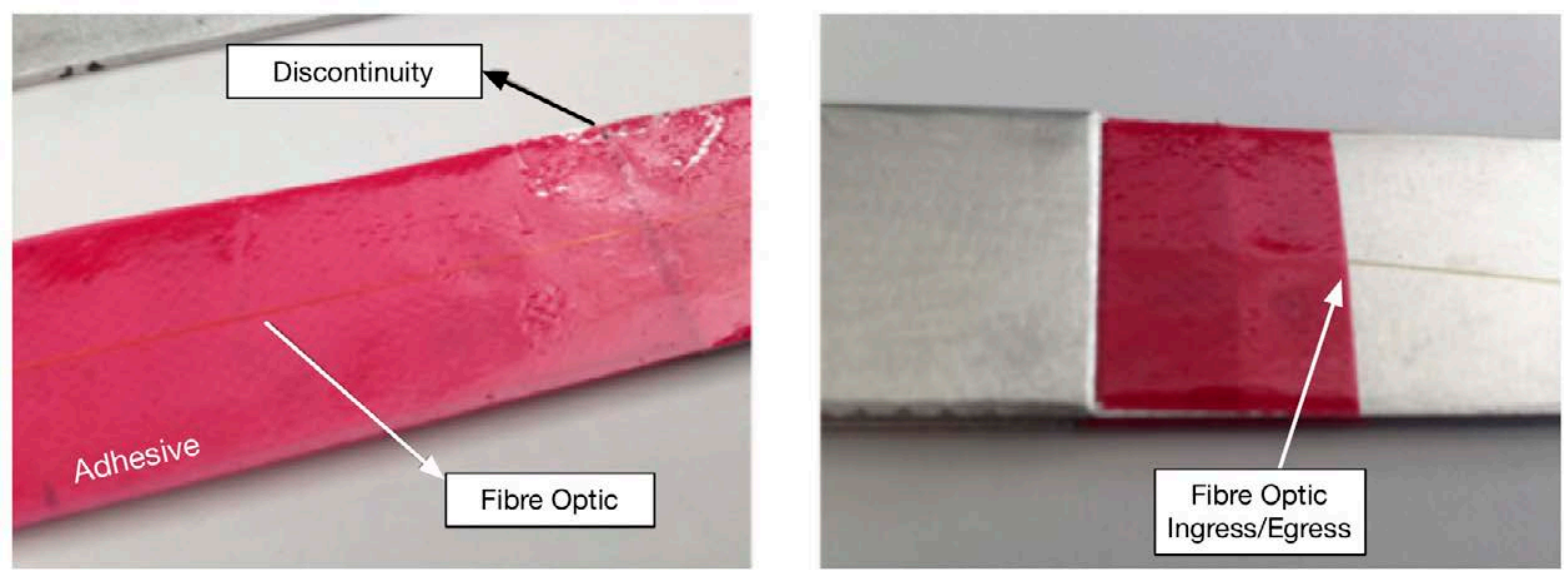

Figure 60. (Left) Optical fibre embedded in the aluminium plate. (Right) Ingress/Egress of the optical fibre with additional adhesive.

The discontinuity of $1 \mathrm{~mm}$ of length at the middle of the specimen can be representative of a flaw in the adhesive joint. The aim of this test is to prove the capability of the distributed strain measurement to detect the strain changes related to this discontinuity.

The considered structural adhesive was film epoxy FM®73 de Cytec. The curing cycle was of 1 hour at $120^{\circ} \mathrm{C}$ and $300 \mathrm{kPa}$ of pressure. This adhesive also has a carrier fabric made of polyester to improve the thickness control all along the overlap area.

Table 6. Properties of aluminium plates and adhesive used for the experimental test.

\begin{tabular}{r|c|c|} 
& Aluminium 2024T3 & Adhesive FM73 \\
\hline $\mathrm{E}[\mathrm{MPa}]$ & 73000 & 2160 \\
\hline$v$ & 0.33 & 0.35 \\
\hline Thickness [mm] & 2 & 0.2 \\
\hline
\end{tabular}

The specimen was held to an MTS machine to introduce a tensile load of $3 \mathrm{kN}, 4 \mathrm{kN}$ and 6 $\mathrm{kN}$. In Figure 61, again the ingress-egress of the optical fibre embedded in the adhesive joint can be appreciated as a red light obtained from a fibre checker. The efforts as a result of the embedded fibre caused light to scatter as it passed through. 


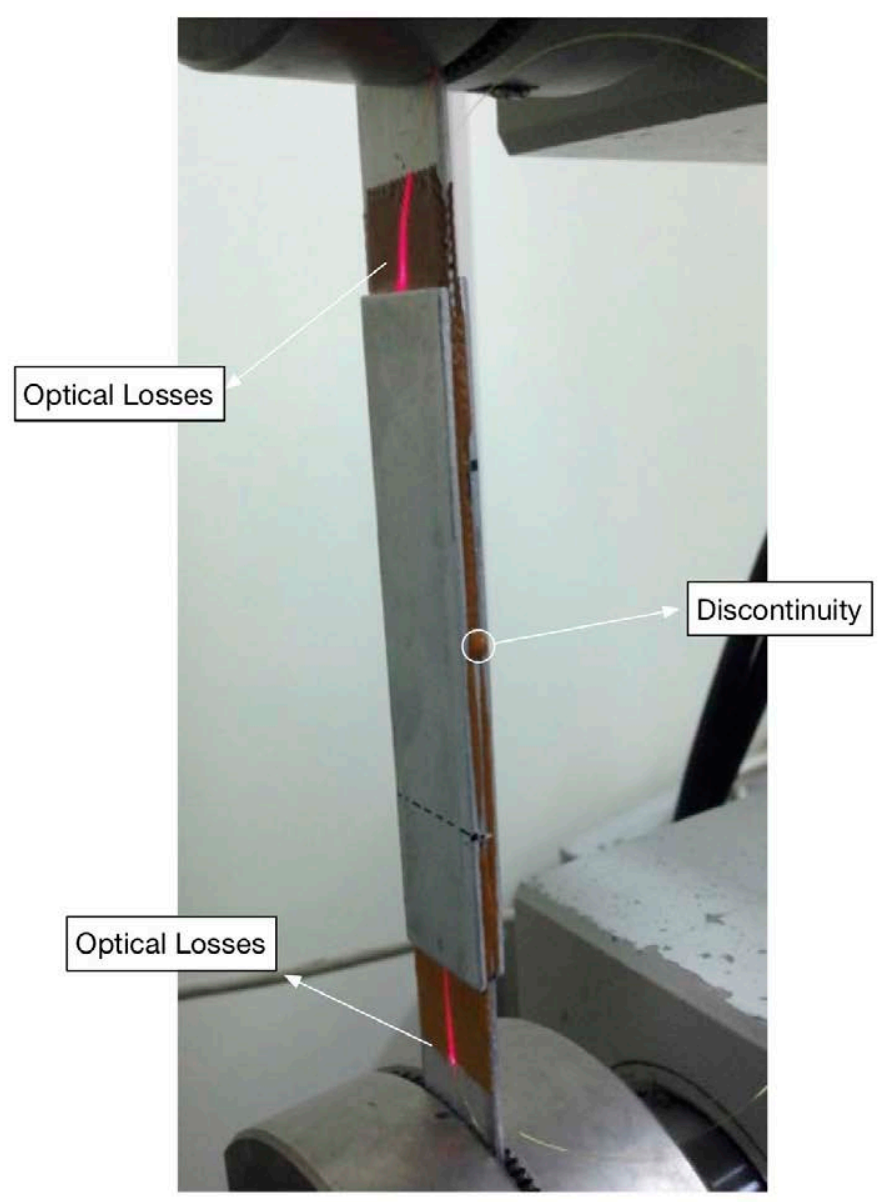

Figure 61. Double lap joint test held in the MTS machine. Optical losses can be appreciated at ingress/egress.

\subsubsection{Experimental Results}

In Figure 62, results from distributed fibre optic sensors are shown for all load steps. In this figure we must draw attention to:

- The discontinuity at the middle of the overlapping area (zone B). In all distributed strain measurements this flaw can be distinguished. From $6 \mathrm{kN}$ of tensile load the strain, the area near the flaw started to become unstable since positive and negative strains in a very small area $(5 \mathrm{~mm})$ can be observed.

- The specimen is loaded in the linear region since the strain level is uniform with the applied load at each step.

- The strain level in the overlap area (zone B) is three times higher than the boundaries of the double lap joint due to thickness distribution.

- There are some single points removed from these measurements as a result of very high noise. This may be caused by the thickness change at this point. 


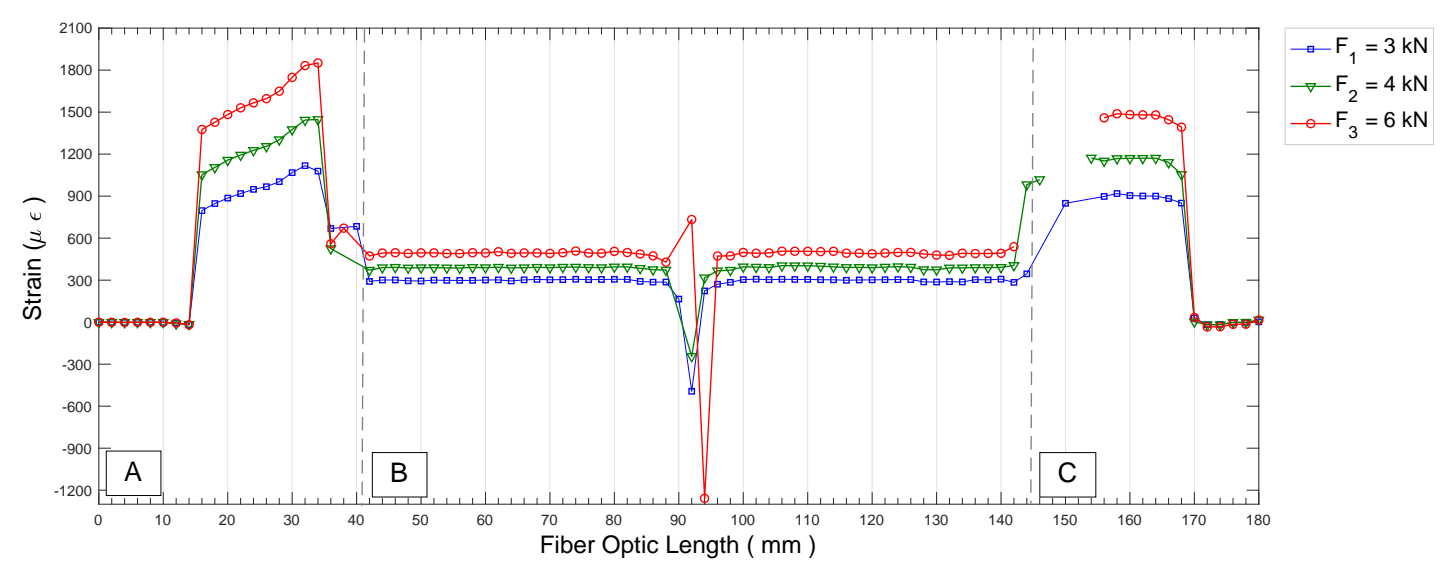

Figure 62. Distributed fibre optic measurement at $3 \mathrm{kN}, 4 \mathrm{kN}$ and $6 \mathrm{kN}$.

In order to compare these results, a finite element model was developed in MSC Patran software. Due to the specimen during the experimental test being loaded in the linear zone, a linear solution (sol101) is used to obtain the results under $3 \mathrm{kN}$ of tensile load (see Figure 63). The discontinuity was also modelized along the cross-section of the coupon of $1 \mathrm{~mm}$ length.

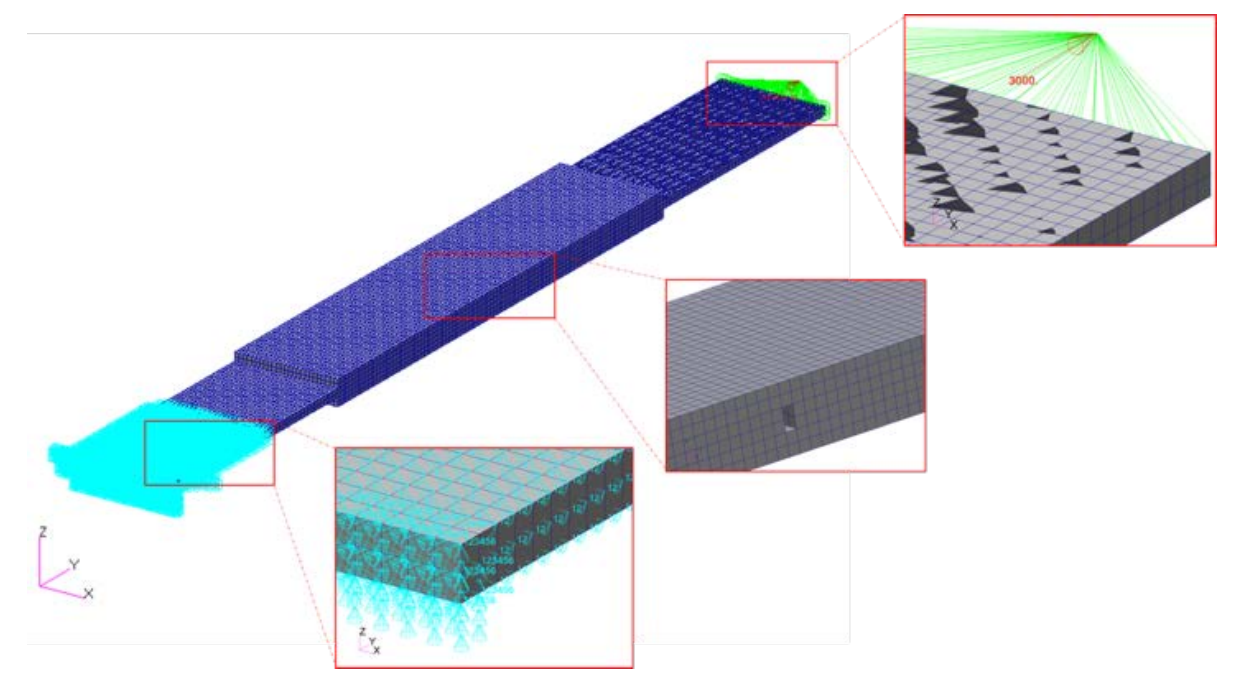

Figure 63. Boundary conditions of FEM for the double lap joint and $3 \mathrm{kN}$ of tensile load.

Afterwards, the results of the linear solution obtained with MSC Nastran are shown in Figure 64. In this figure, the resultant strain field is plotted along the length of the specimen (Y-axis). As expected, the highest strain values were obtained at the surrounding area of the defect. Also, the thickness relationship was also maintained similar to the case of the distributed strain measurement. 


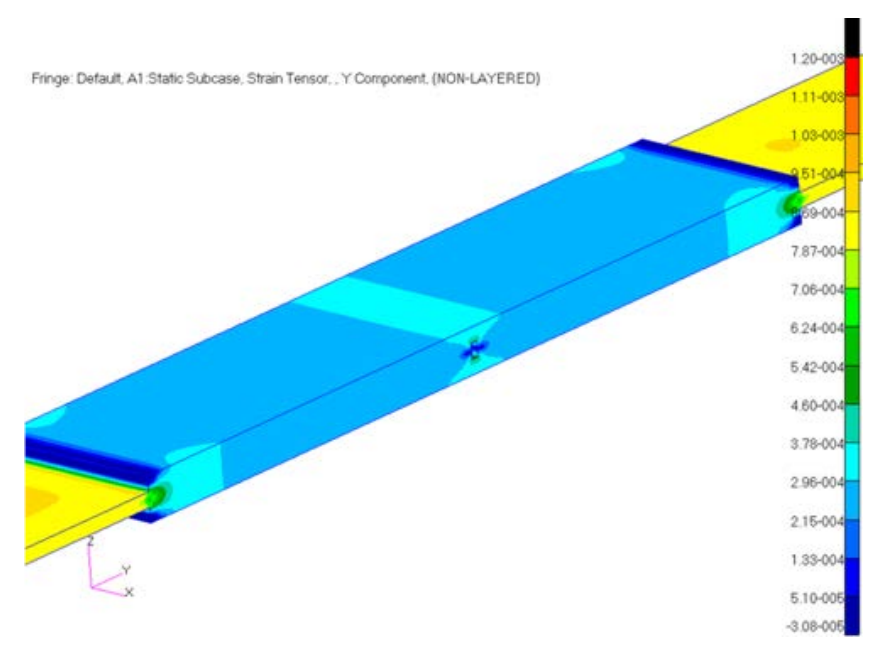

Figure 64. FEM results for the double lap joint under $3 \mathrm{kN}$ of tensile load.

Finally, in Figure 65, a comparative of distributed strain measurement and FEM was performed in the longitudinal direction, i.e. the optical fibre direction. These results show a good fitting agreement in the strain level recorded at each zone of the joint, however the influence of the defect is more significant in the distributed measurement than in the FEM measurement.

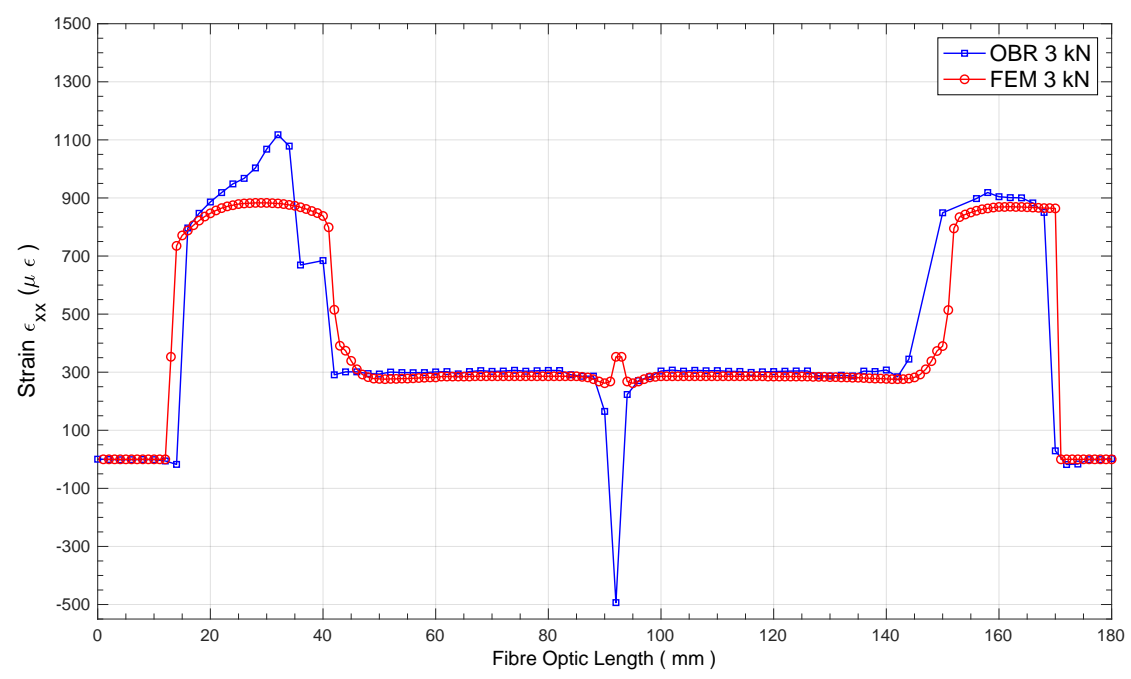

Figure 65. Comparative of FEM results and distributed strain measurement under $3 \mathrm{kN}$ of tensile load.

\subsubsection{Conclusions of Double Lap Joint}

From a fibre optic line placed on the overlap area, full-field strain could be successfully monitored. Furthermore, a fitting agreement was obtained related to the FEM results. The main advantage achieved by the use of the optical fibre lies in capability of measuring the presence of flaws in the adhesive at the near strain field. If the optical fibre is placed close to 
the flaw, it is able to detect changes over the distributed strain measurement. However, two main aspects should be further investigated:

- The capability of detecting changes in the distributed strain measurement by the presence of flaws in the far-field.

- The quality of the bonding due to the inclusion of the optical fibres inside the adhesive layer.

- Survivability of the embedded optical fibre under different load requirements. This is a major issue to take into account since the embedded optical fibres cannot be repaired and, in such case, all the adhesive would have to be removed in order to install a new optical fibre.

Despite, these issues, DFOS techniques have been proven to be a good candidate to monitor the reliability of the joints with a single optical fibre with an easy integration.

\subsection{Free-Edge Delamination Damage}

This experimental test is focused on the analysis of the residual strain field. These measurements give valuable information in case of any damage that has been produced in the monitored structure for different time intervals. For this purpose, the fibre optic sensing network was located at the free-edge of different CFRP coupons since these areas are prone to suffer accidental damages.

Some of these results and figures can be found in the conference paper "Free-Edge delamination location and growth monitoring with an embedded distributed fiber optic network" (Patricia F. Díaz-Maroto, Fernández, Larrañaga, \& Guemes, 2016).

\subsubsection{Introduction and Experimental Motivation}

One of the most common damage mechanisms in composite materials is the delamination appearance. The onset of a delamination is not always easily detected attributable to the possibility of it being produced internally. During the manufacturing and assembly processes of the composite structures, several low velocity impacts can occur and also during its life service in the maintenance plan (Hocheng \& Tsao, 2003; Long, Yao, \& Zhang, 2015; Malhotra \& Guild, 2014). These impacts usually occur near the free-edges of the structures, which have been widely investigated by several authors (Guillamet, Turon, Costa, \& Linde, 2016; Malhotra, Guild, \& Pavier, 2008). These research herein mentioned attempt to describe the delamination onset of the edge which is mainly dominated by interlaminar shear stress, though it may be triggered by the normal interlaminar stress. Furthermore, another issue to consider is that stress loads can be concentrated easier near free-edge of composite laminates where impacts are also expected to occur. Therefore, these stresses prompt a debonding between adjacent plies and determine the delamination onset in the sequence orientation change (Patricia F. Díaz-Maroto, Fernández, Larrañaga, et al., 2016). 
Finally, the goal of the free-edge experiment is to detect accidental impact damage at an early stage by using the residual strain field of a dense distributed fibre optic network located on the free-edge of the specimens. For this purpose, different configurations of composite laminates were manufactured by hand lay-up. Afterwards, they were impacted by using a drop-weight testing machine at CCMSS. As stated above, not only the goal of this experiment lies in detect local strain changes from the residual strain field, but also to study the influence of the two configurations of low velocity impact tests. In general terms, this research tries to be representative for detection of free-edge delamination in aircraft composite structures such as man-holes, which are vulnerable to endure this damage.

\subsubsection{Experimental Setup}

In order to prone an early delamination damage, a cross-ply lay-up was selected to manufacture all specimens. The dimensions of the specimens were $200 \mathrm{~mm}$ long and $80 \mathrm{~mm}$ wide, made of carbon/epoxy prepreg AS4/8554 by Hexcel ${ }^{\circledR}$. The ply thickness was of 0.15 $\mathrm{mm}$.

All distributed sensing networks were positioned near the free-edge of the different specimens where delamination damage was expected to occur. Likewise, different paths that separated one another by $5 \mathrm{~mm}$ were placed parallel to the edge up to perform 6 paths, i.e. 30 $\mathrm{mm}$ length of cover area. As seen in Table 7, specimen no. 3 had the optical fibre embedded in the first-ply (opposite impact surface). For that reason, polyimide coating fibre was used on this specimen due to its higher temperature resistance and smaller diameter than acrylate coating, which was used for the rest of the specimen. Polyimide coating ensures the optical fibre will not be degraded during curing process (see Figure 66). For the other specimen optical fibre was bonded directly to the cured surface with cyanoacrylate.

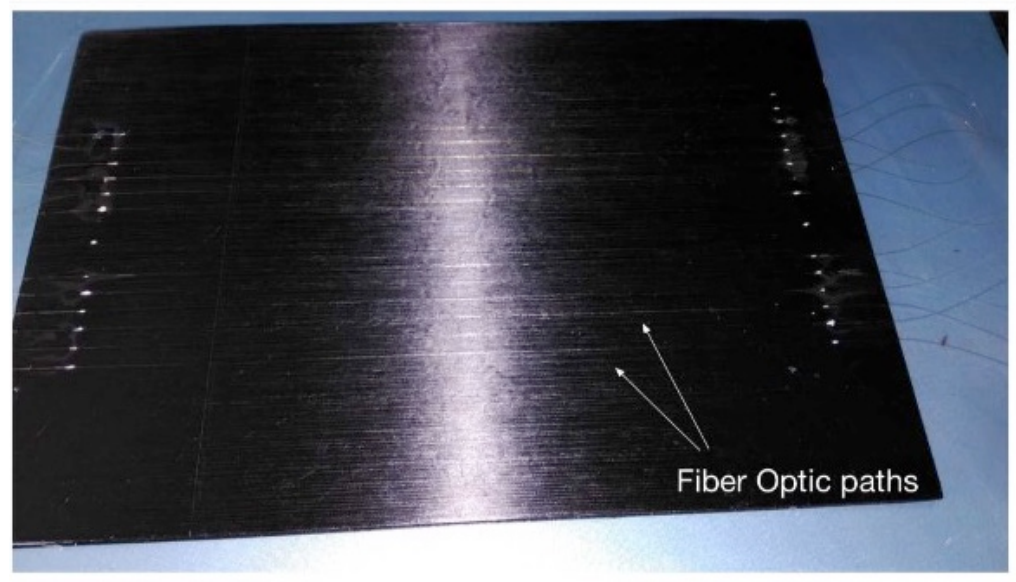

Figure 66. Positioning of polyimide fibre optic during specimen manufacturing.

A drop weight testing machine was used to carry out the delamination for all specimen under different energy levels by adjusting the height of the impactor. The mass of this impactor was of $5 \mathrm{~kg}$. 
Depending on the impact location, on-edge or near-edge, boundary and constraint conditions of different specimen varied. On one hand, on-edge specimens were fixed with clamps on both sides to perform the impact in normal direction to the edge Figure 67a. On the other hand, near-edge specimens were fixed with metallic plates along the perimeter of the selected impact area but leaving the free-edge without constraint Figure 67b.
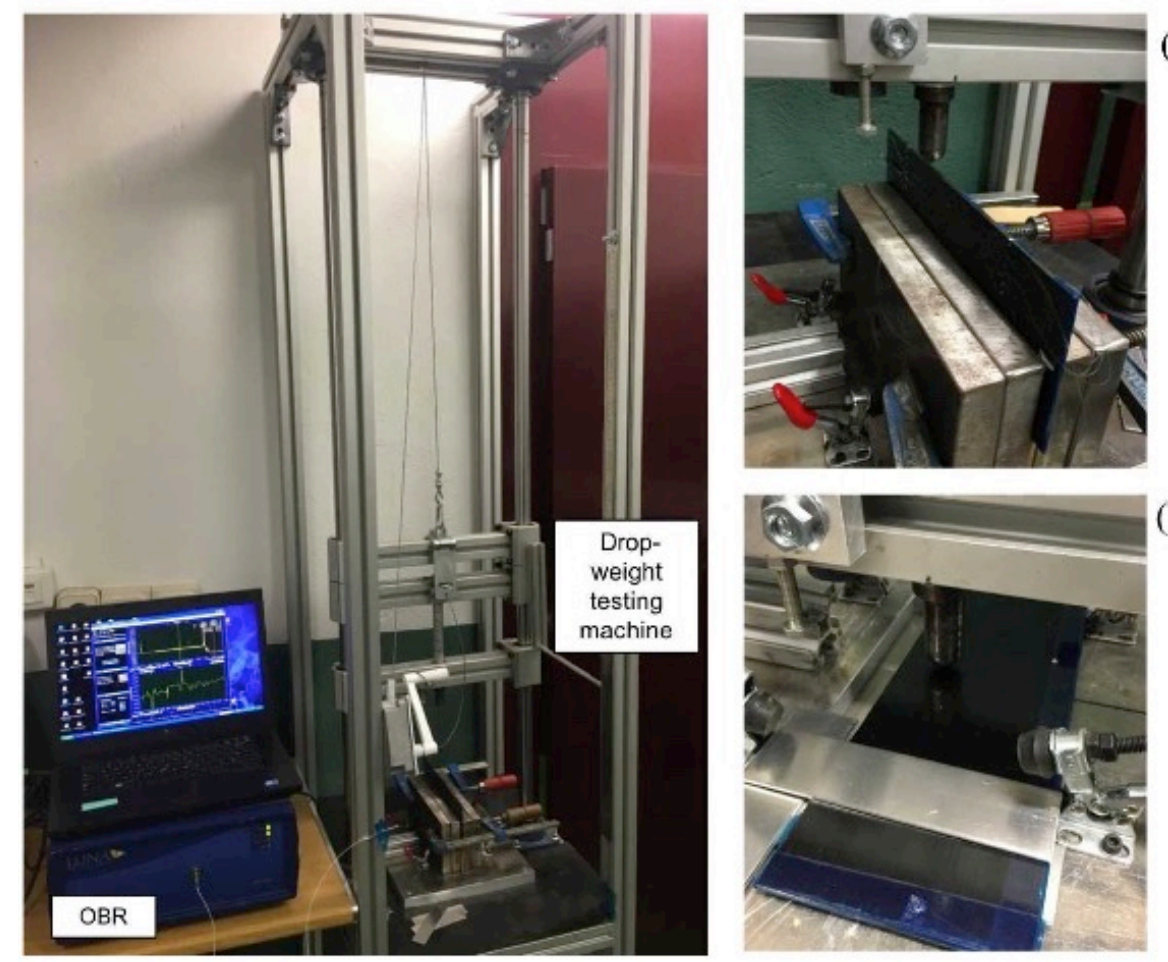

(a)

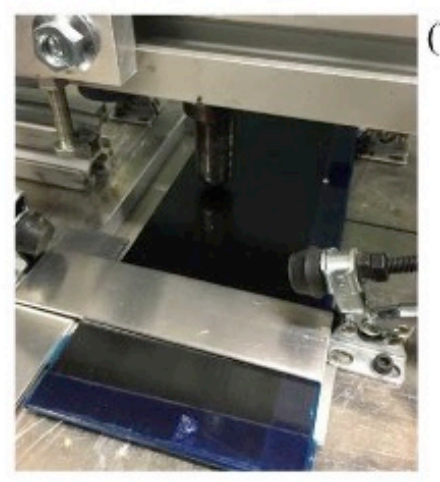

(b)

Figure 67. Drop-weight testing machine. (a) On-edge impact. (b) Near-edge impact.

Table 7 summarizes all specimen configurations and impacts carried out through the different low impact tests.

Table 7. Summary of tested specimens.

\begin{tabular}{|c|c|c|c|c|}
\hline Specimen no. & $\begin{array}{c}\text { Stacking } \\
\text { Sequence }\end{array}$ & $\begin{array}{c}\text { Impact } \\
\text { Energy }[J]\end{array}$ & $\begin{array}{c}\text { Impact } \\
\text { Location }\end{array}$ & $\begin{array}{c}\text { FO Network } \\
\text { Location }\end{array}$ \\
\hline 1 & {$\left[0_{4} / 90_{4}\right] \mathrm{s}$} & {$[1,2,3,4,5]$} & On-edge & Both surface side \\
\hline 2 & {$\left[0_{2} / 90_{2}\right] \mathrm{s}$} & {$[1.7,5,5.5]$} & On-edge & One surface side \\
\hline 3 & {$\left[0_{4} / 90_{4}\right] \mathrm{s}$} & {$[2,3,5.8,6,8]$} & Near-edge & Embedded first-ply \\
\hline
\end{tabular}

A schema of the configuration setup is shown in Figure 68. 

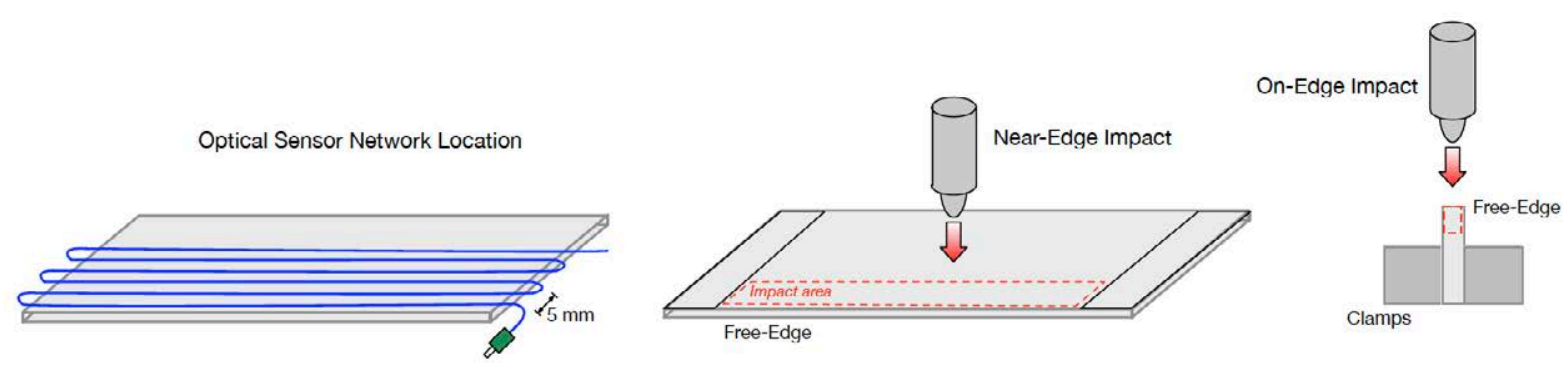

Figure 68. Schema of different impact configurations and disposition of fibre optic network bonded close to the near edge made up 6 paths separated $5 \mathrm{~mm}$ each other.

\subsubsection{Experimental Results}

When delamination damage occurs, very small changes can be appreciated over the length of the distributed strain measurement. This happens when delamination damage causes lamina debonding of a delimited area which becomes in permanent residual strains in the laminate. In some cases, these changes are not easily detectable, but the accuracy of the FOS allows to identify these sensing points. Distributed strain measurements were acquired after each impact, including the baseline measurement, so the strain field that can be observed corresponds to the residual strain field. If a perfect strain transfer is considered from the laminate to the optical fibre, every strain change was assumed to be delamination damage onset.

In the next paragraphs, all distributed fibre optic results are presented and also compared to NDT ultrasonic C-Scan technique.

\subsubsection{Specimen n0.1-On-Edge}

As shown above in Table 7, this specimen was instrumented with optical fibre lines by both sides. The aim of this specimen is to give an analysis of the influence of damage delamination onset related to both sides.

Figure 69 shows the whole distributed strain measurement for specimen n0. 1. It can be observed in this figure that residual strain level after each impact is very low around $\pm 10 \mu \varepsilon$. In addition, out of these bounds some high peaks of strain are noticeable at $2.2 \mathrm{~m}$ and $2.5 \mathrm{~m}$ of fibre optic length and also, in some other locations. 


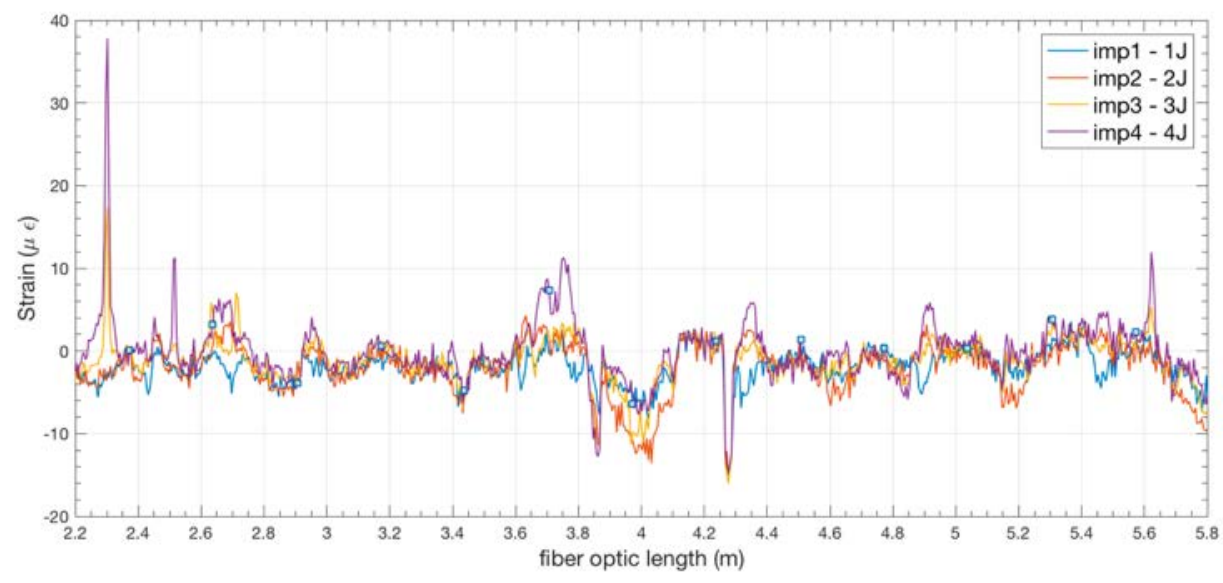

Figure 69. Whole distributed strain measurement from impacts from $1 \mathrm{~J}, 2 \mathrm{~J}, 3 \mathrm{~J}$ and $4 \mathrm{~J}$.

More in detail in Figure 70, the evolution of residual strain after each impact can be appreciated. At 2J of impact energy no apparent residual strain was recorded by the different paths. Path 1 ( $5 \mathrm{~mm}$ deep) recorded residual strain of $15 \mu \varepsilon$ in a length of $20 \mathrm{~mm}$ at 3J. Path 2, separated $5 \mathrm{~mm}$ from Path 1 , is not able to detect any changes in strain field. Nevertheless, at $4 \mathrm{~J}$ of impact energy, delamination depth achieved path 2 which could record $12 \mu \varepsilon$ of residual strain, while path 1 damaged recording is visible up to $35 \mu \varepsilon$ of residual strain. It should be noted that delamination damage appearance causes very small-scale changes in the strain field and they are not easily detectable without a detail inspection (such as NDT or visual inspection). The use of optical fibres to notice these small changes in the structure provide valuable information to detect this damage in an early stage.
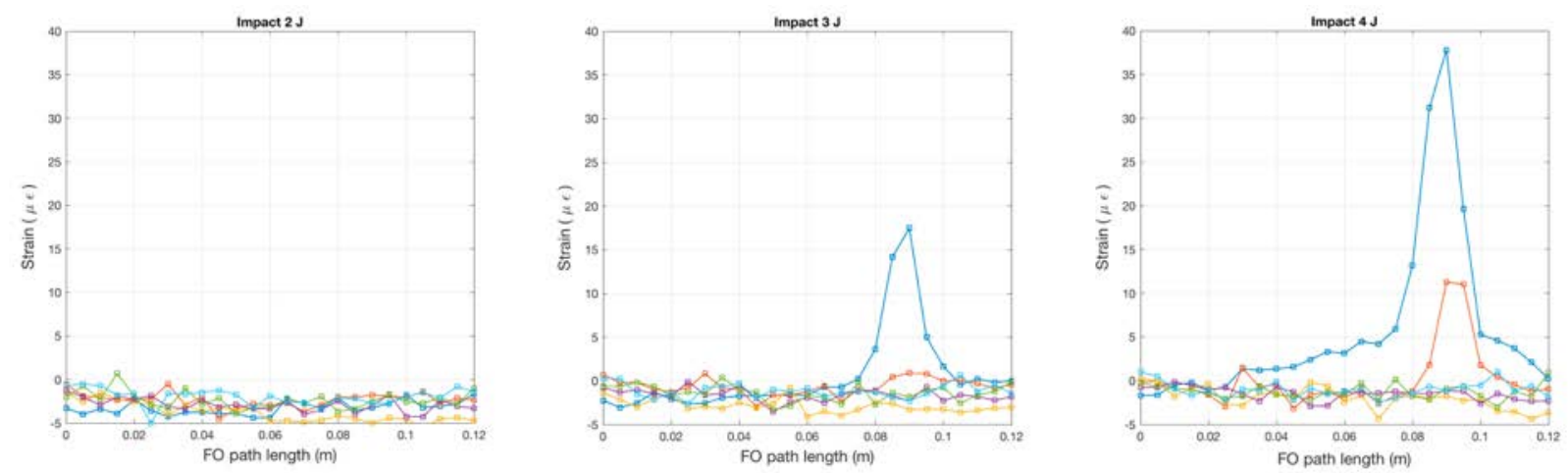

$\rightarrow-$ Path $1.5 \mathrm{~mm} \rightarrow$ Path $2-10 \mathrm{~mm} \rightarrow$ Path $3-15 \mathrm{~mm} \rightarrow-$ Path $4-20 \mathrm{~mm} \rightarrow-$ Path $5-25 \mathrm{~mm} \rightarrow-$ Pathe $-30 \mathrm{~mm}$

Figure 70. Residual strain evolution after each impact from 2J, 3J and 4J for specimen n0.2 face A.

Same behaviour was recorded in face B of the specimen n0.1, see Figure 71. The main difference lies in the residual strain level which is considerably lower than face A. The maximum value reached is $12 \mu \varepsilon$ against $35 \mu \varepsilon$ of maximum value for face $\mathrm{A}$. The reason for this is the debonding of the lamina occurred in the interface closer to face A since stacking sequence is $\left[0_{4} / 90_{4}\right]$ s. 

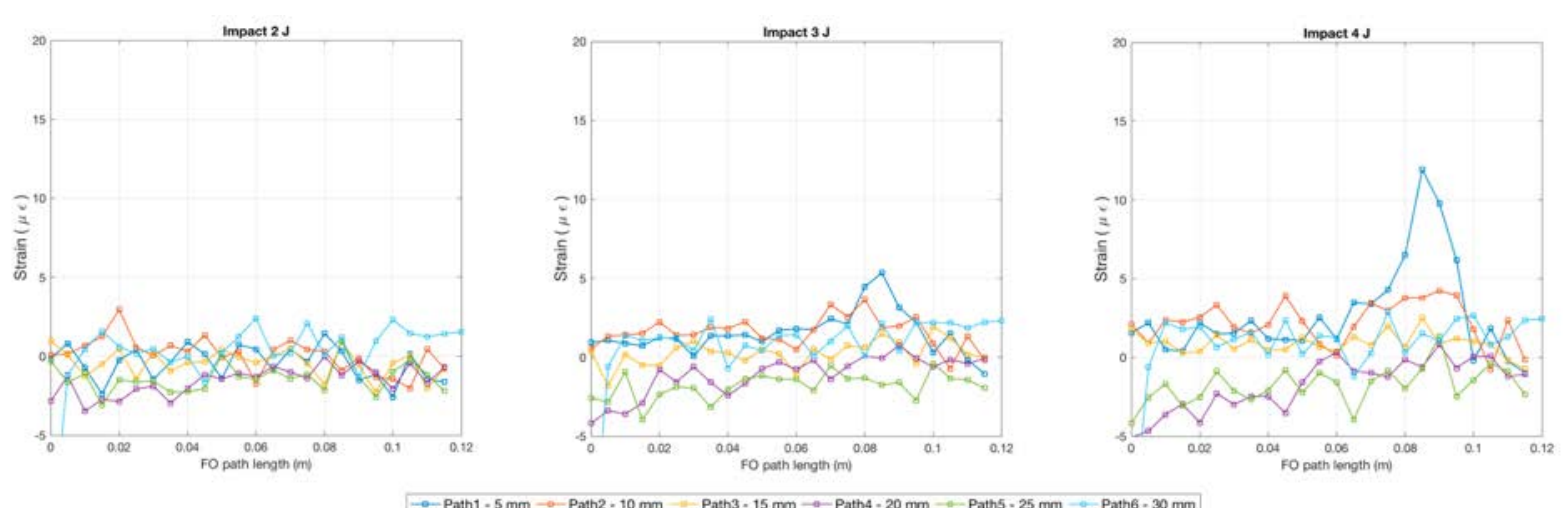

Figure 71. Residual strain evolution after each impact from 2J, 3J and 4J for specimen n0.1 face B.

In Figure 72, a distributed measurement map is reconstructed to visualize the evolution of the delamination at different impacts of energy. As explained above, delamination occurred in the interface close to face of the sensing network and therefore, the residual strain level is higher in this side.
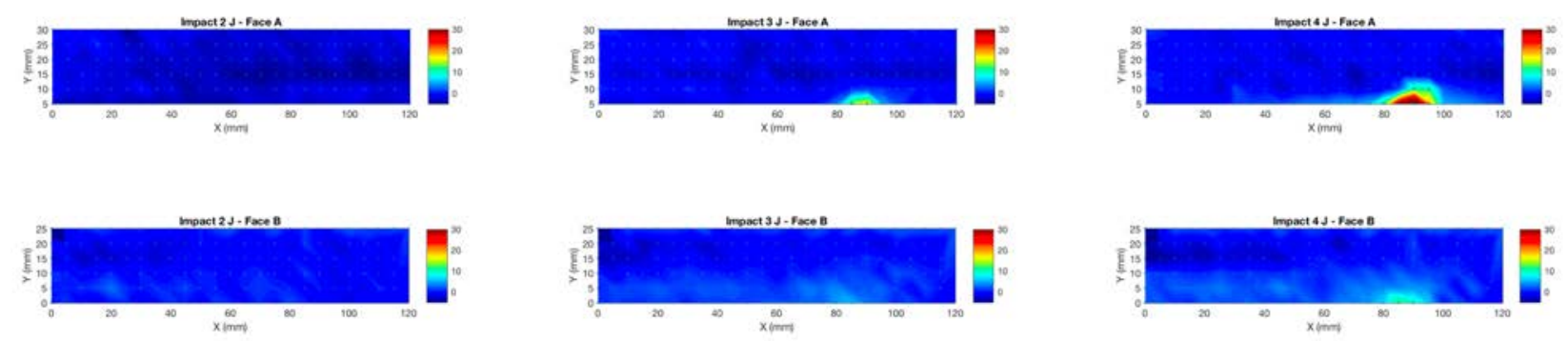

Figure 72. Strain colour map on both faces (A and B).

After $4 \mathrm{~J}$ of impact energy over the specimen, permanent damage was caused on the freeedge and as a consequence the optical fibre line was broken.
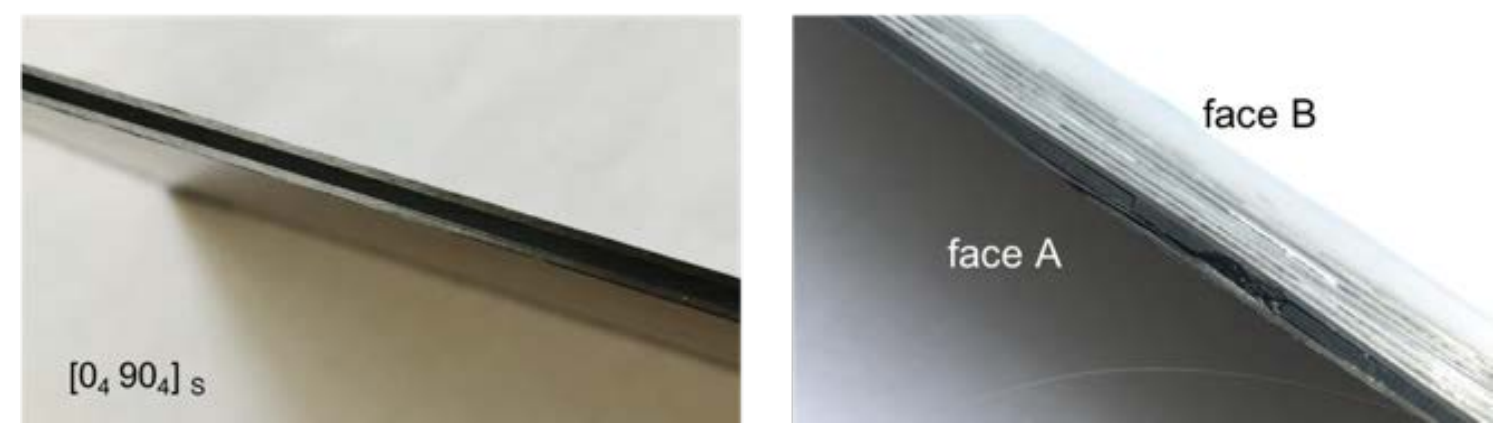

Figure 73. (Left) Specimen 2 before impact test n0.1. (Right) Specimen n0.1 after 5J of impact energy. Lamina debonding appeared on face A side. 


\subsubsection{Specimen n0. 2 - On-Edge}

Same results were obtained in specimen n0.2. In this case, only one side was monitored. The main difference related to specimen $\mathrm{n} 0.1$ lies in all lamina was debonded as can be seen in Figure 74 since permanent residual strain was recorded all along the length of the path 1 (at 5 $\mathrm{mm}$ from free edge).
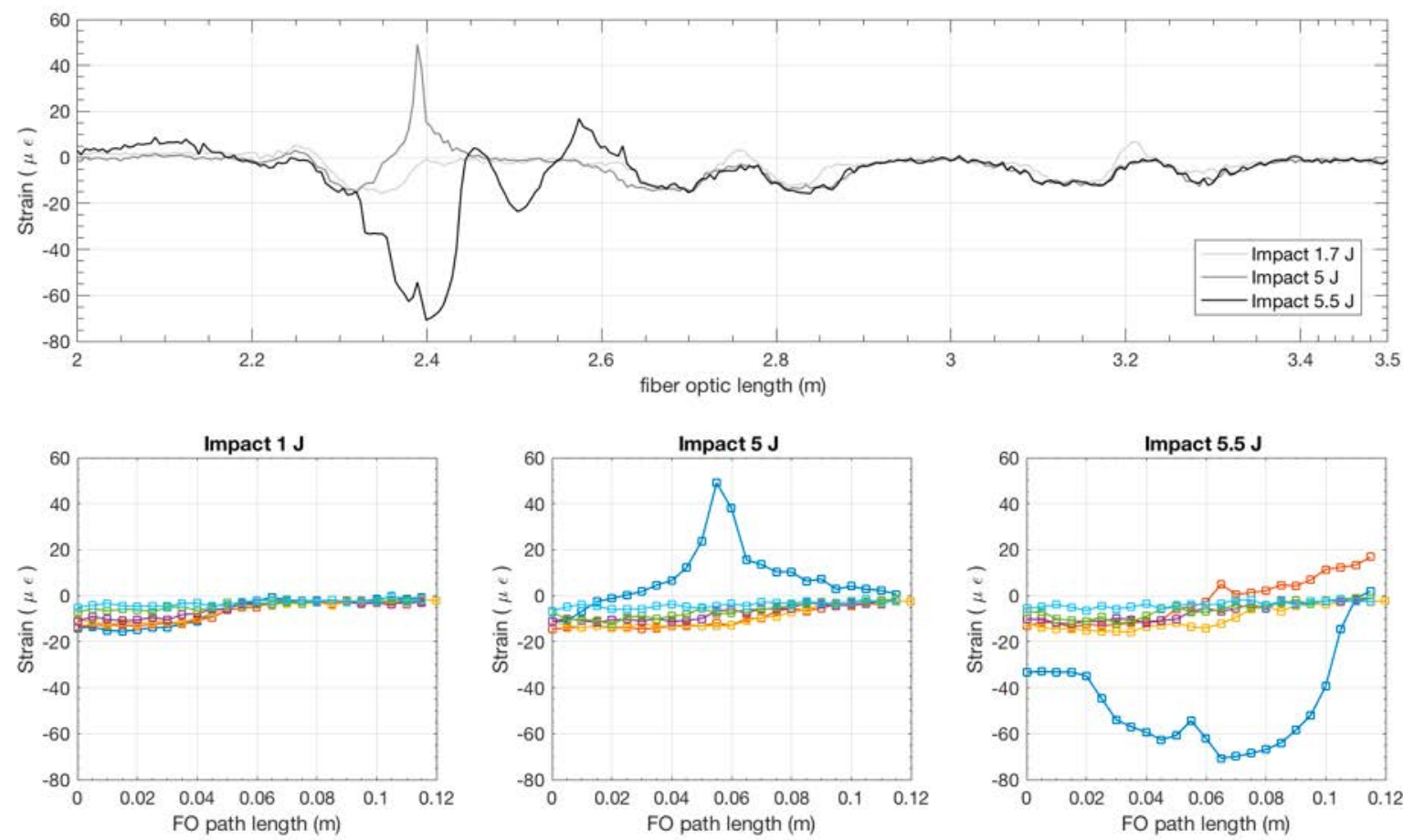

Q-Path1 - $5 \mathrm{~mm} \rightarrow$ Path2 $-10 \mathrm{~mm} \rightarrow$ Path3 - $15 \mathrm{~mm} \rightarrow$ Path4 - $20 \mathrm{~mm} \rightarrow$ Path5 - $25 \mathrm{~mm} \rightarrow$ - Path6 - $30 \mathrm{~mm}$

Figure 74. All distributed fibre optic measurement for specimen n0.2.

The stack sequence of this specimen was also different from specimen n0.1. This can be prone to more catastrophic lamina debonding regarding the residual strains recorded in both experiments.

\subsubsection{Specimen n0.3-Near-Edge}

The impact in specimen n0.3 was performed around the path 4 on the opposite side. Hence, path 3 and path 5 are expected to be more sensitive to the residual strain field caused by the impact. In Figure 75 whole distributed measurement is displayed for the three impacts. As observed in this figure, it can be noticed in the centre of path 4 which is coincident with the impact location on the opposite side the strain field recorded is under compression due to impact. On both sides of this location, path 3 and 5 presented same behaviour with the slight difference that path 3 recorded higher strain level. This is the typical behaviour for internal delamination damage. 

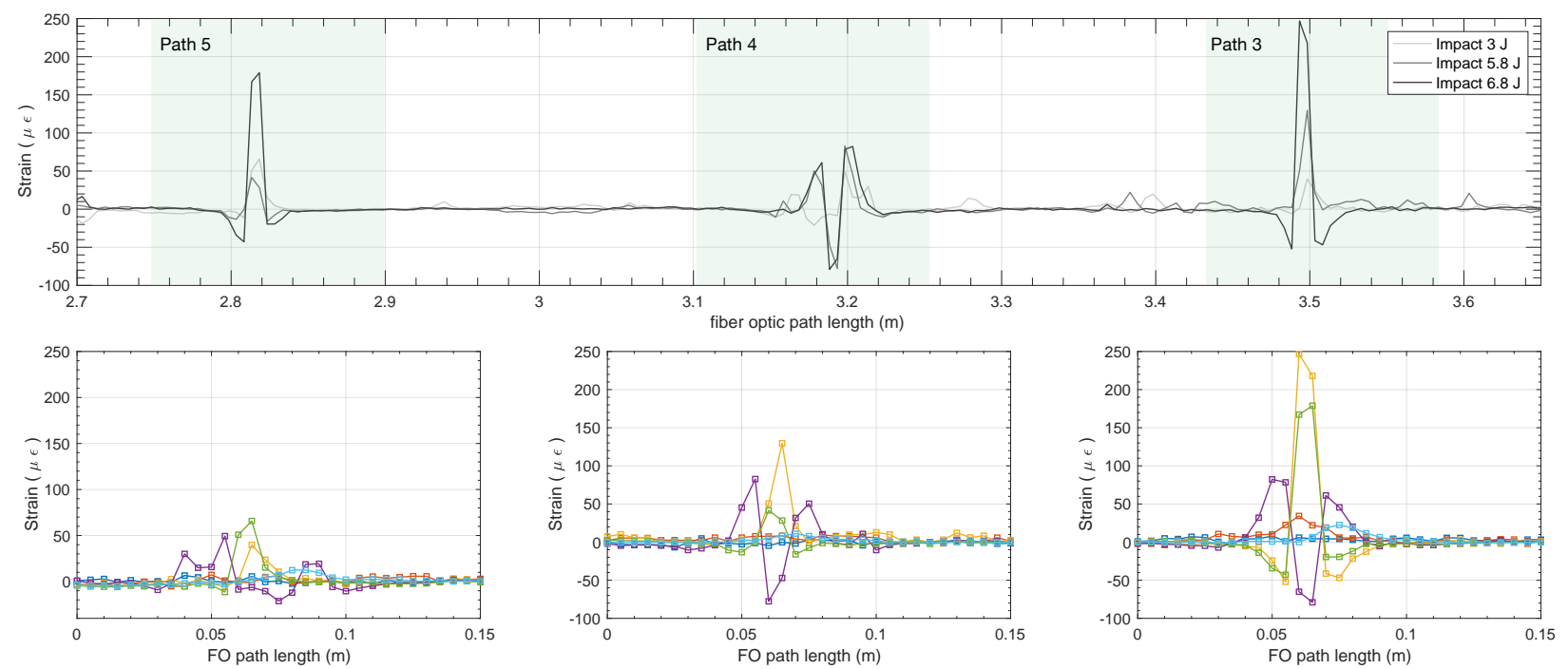

$\rightarrow$ Path1 - $5 \mathrm{~mm} \rightarrow$ Path2 - $10 \mathrm{~mm} \rightarrow-$ Path3 - $15 \mathrm{~mm} \rightarrow$ Path4 - $20 \mathrm{~mm} \rightarrow$ Path5 - $25 \mathrm{~mm} \rightarrow$ - Path6 - $30 \mathrm{~mm}$

Figure 75. All distributed fibre optic measurement for specimen n0.3- delamination near edge.

In Figure 76, the distributed strain map is represented for this impact. On the one hand, it should be pointed out in this figure how the position of the impact is under compressive load since in this area the fibre was extended. On the other hand, the near area also experienced tensile load as a consequence of the impact. From $3 \mathrm{~J}$ impact, the residual strain was recorded after the impact where it can be appreciated.
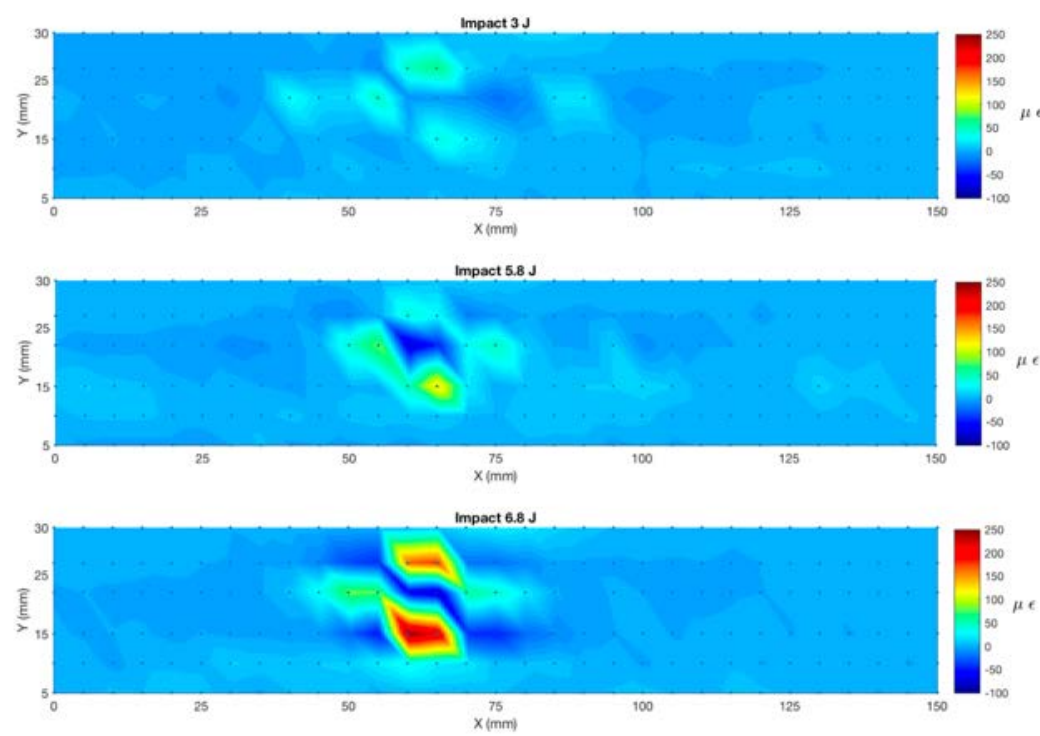

Figure 76. 3D residual strain map for specimen n0.3

This is the most typical delamination shape in cross-ply laminates, so-called "peanut shape". Therefore, this shape can be roughly observed after the impact test of this coupon. It can be 
noted that in this case the optical fibre was embedded in the last composite layer (opposite side on the impact) in a crooked configuration. For this configuration, the ultrasonic inspection could be carried out since both faces were free of optical fibres. In Figure 77, the C-Scan inspection can be observed after a $6.8 \mathrm{~J}$ of energy impact. The damaged area due to the presence of a delamination can be observed in between $50 \mathrm{~mm}$ and $70 \mathrm{~mm}$ along the freeedge of the specimen. As it was expected, the delamination damage appeared on the opposite face of the impact side.

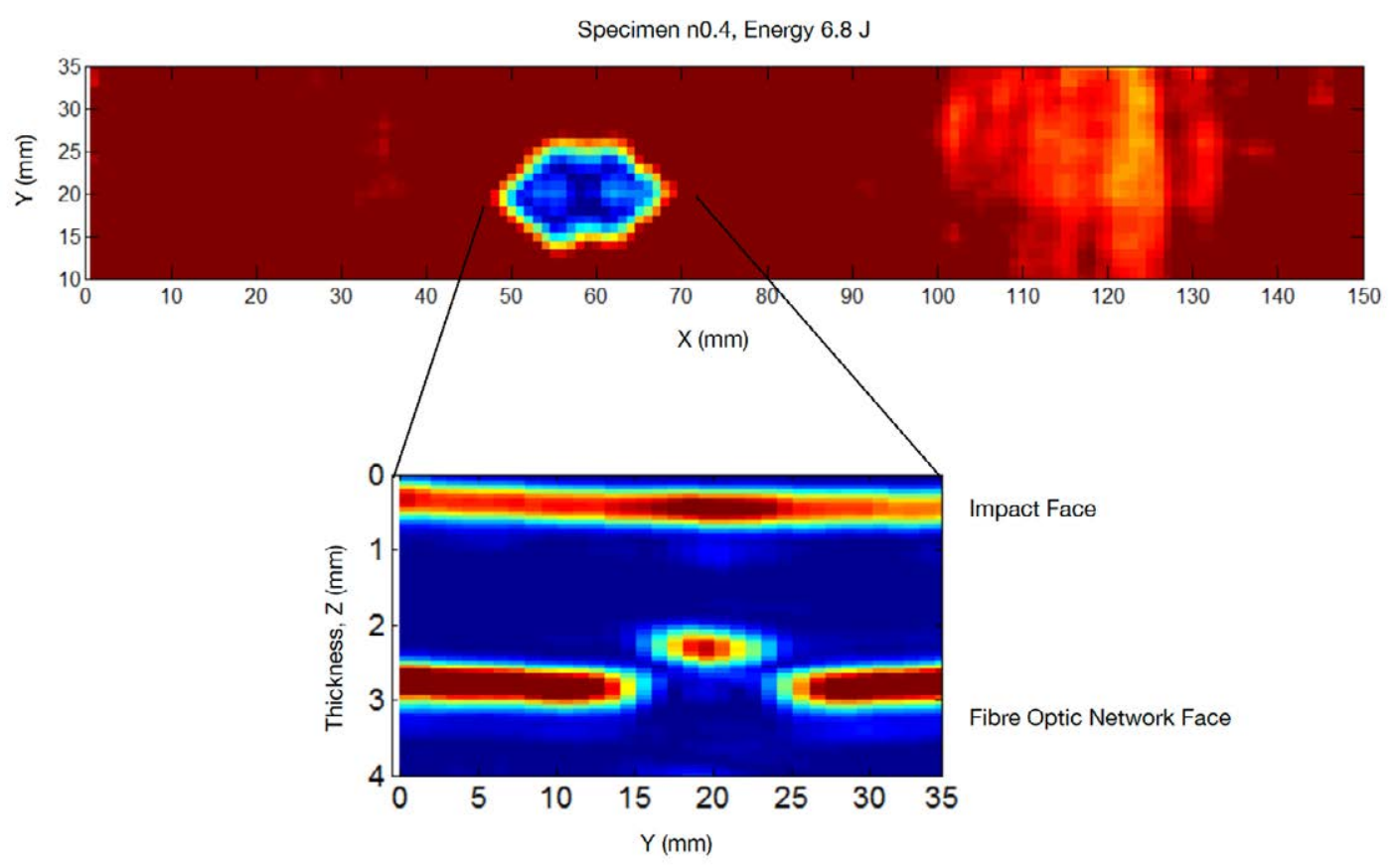

Figure 77. NDT results for the specimen n0.3 after $6.8 \mathrm{~J}$ of impact energy.

\subsubsection{Conclusions of Free-edge test.}

It has therefore been proven throughout this experiment that the residual strain of the distributed optical network gives valuable information about the real structural state of the structures. The strain accuracy of this technique is able to detect very small changes which can be considered as an internal damage in the composite materials.

As expected, the on-edge impacts on the free-edges are more critical than near-edge since a complete lamina was debonded for a $5 \mathrm{~J}$ impact energy. However, the highest residual strain values were recorded in near-edge type impacts, and also a larger number of paths were affected. Nonetheless, the damage is visible as shown in Figure 73, only the first path could detect the strain change.

Regarding the results of the NDT a fitting agreement could be observed, not only in the location of the delamination, but also in the approximate shape of the affected area. 


\section{Chapter 6 GLOBAL DAMAGE DETECTION APPROACH}

This chapter focuses on global damage detection in a real aircraft structure. Particularly, global damage detection approach was conducted in two stiffened composite panels. The analysis of these panels involves three steps: damage monitoring, fatigue life and buckling. The main goal of this experiment is to analyse the artificial damages and, detect and locate buckling appearance in an early stage prior to the catastrophic failure. Some ideas and figures shown in this chapter have appeared previously in the full-length article titled "Buckling detection of an omega-stiffened aircraft composite panel using distributed fibre optic sensors" (Patricia F. Díaz-Maroto et al., 2018).

\subsection{Introduction and Experimental Motivation}

The global damage detection approach was carried out in two stiffened composite panels. The selection of this type of panel lies in the challenge of reducing the weight in aircraft structures, which has been nearly settled through the application of composite materials (Bai, Lei, Wei, Tao, \& Yan, 2017; Boyer \& Padmapriya, 2016; Dutton, Kelly, \& Baker, 2004). Thus, most innovative commercial aircrafts, such as the A350, are made up of $50 \%$ composite materials in its structure and components: horizontal stabilizer, fuselage sections, or wing panel (Gay, 2015).

Therefore, in the quest to enhance the strength-to-weight ratio, stiffened composite panels have been a breakthrough in the area of structural configurations, since they improve the load bearing, increasing the stiffness and panel stability. However, one of the most important drawbacks related to this configuration is that they are prone to buckling failure because the panel skin is generally thinner. Furthermore, this failure is very likely to occur under an inplane shear load, causing diagonal tension on the panel skin between the stiffeners and half buckling waves, which create a specific angle with the stiffener axis (Kassapoglou, 2013).The onset of high shear loads in a stiffened panel may incur not only on the appearance of global and local buckling, but also damage, such as delamination or stiffener de-bonding (Aydogdu \& Aksencer, 2018; Mallela \& Upadhyay, 2006; Xin et al., 2017).

To ensure the in-service safety and stability, stiffened panels are usually tested under a diagonal compressive load, which rapidly induces non-linear behaviour in the panel skin 
owing to a non-pure shear loading. Therefore, an outstanding part of such a panel design is to analyse the buckling response under critical shear loads.

Thus far, back-to-back strain gauge rosettes are the most common strain sensors used to detect the initiation of buckling at a single location (where the sensor is positioned). By comparing two rosette readouts bonded on opposites sides of the structure's skin at the same location, buckling can be predicted when the slopes of their load-strain curve change their direction (Zhu, Yan, Chen, Tong, \& Wang, 2015). The main problem related to a strain gauge sensor is that they can only perform a single point measurement and, furthermore, the wires of these sensors will increase the weight of the structure. Non-contact optical techniques, such as a digital image correlation (DIC) are also usually employed to identify buckling patterns and the full-field deformation of the structure with very high accuracy and precision. However, the cameras used to acquire the images need to be carefully placed and calibrated before testing, and are focused in a specific area. This has led to the application of this technology to be restricted mainly to laboratory testing. Other fringe projection techniques, such as a shadow Moiré technique, is able to measure the out-of-plane displacement and buckling wave evolution (Jin, Ha, \& Goo, 2014). Despite being a good technique to measure the displacement of large structures, it has low measurement accuracy and resolution, which is a requirement in the aeronautical field.

The scope of this experiment involves a novel methodology to detect and locate the local and global buckling in a stiffened composite panel by means of a second derivative analysis of a distributed strain measurement (4.5.2).

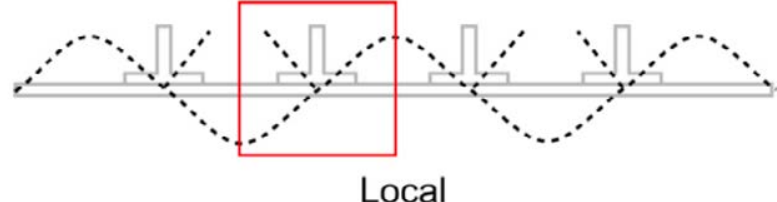

Local

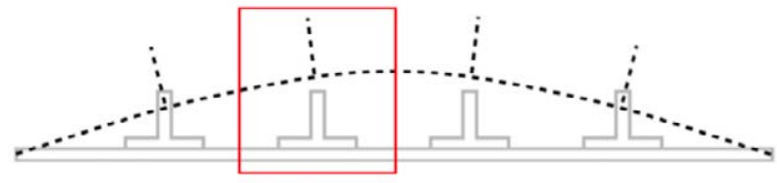

Global

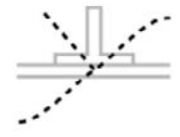

Local

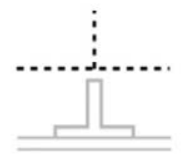

Global

Figure 78. Local and global buckling in stiffened panels (Coburn, Wu, \& Weaver, 2014).

For this purpose, two composite stiffened panels were manufactured representative of a cockpit fuselage skin stiffened by two omega stiffeners plus two fastened metallic frames. A dense distributed sensing network was bonded to the flat panel side, which was also monitored by back-to-back strain gauge rosettes and a high-speed-camera. Prior to conduct the buckling test, different damages were introduced to the panel in order to analyse the damage growth under two fatigue lives. Furthermore, the presence of these damages had an influence in the buckling appearance for both panels which deserves further discussion. 


\subsection{Experimental setup}

\subsubsection{Specimen Description}

The configuration of the two stiffened panels is shown in Figure 79. The panel skin was made of carbon/epoxy unidirectional tape, prepreg AS4/8552, and stiffened using two secondary bonded carbon/epoxy unidirectional tape prepreg AGP280/8552 omega stiffeners (S1 and S2), plus two fastened metallic frames (F1 and F2) made of aluminium. The nominal width and length were $600 \mathrm{~mm} 1655 \mathrm{~mm}$, respectively. The total panel skin thickness was 2.3 $\mathrm{mm}$, and for omega stiffeners it was $1 \mathrm{~mm}$.

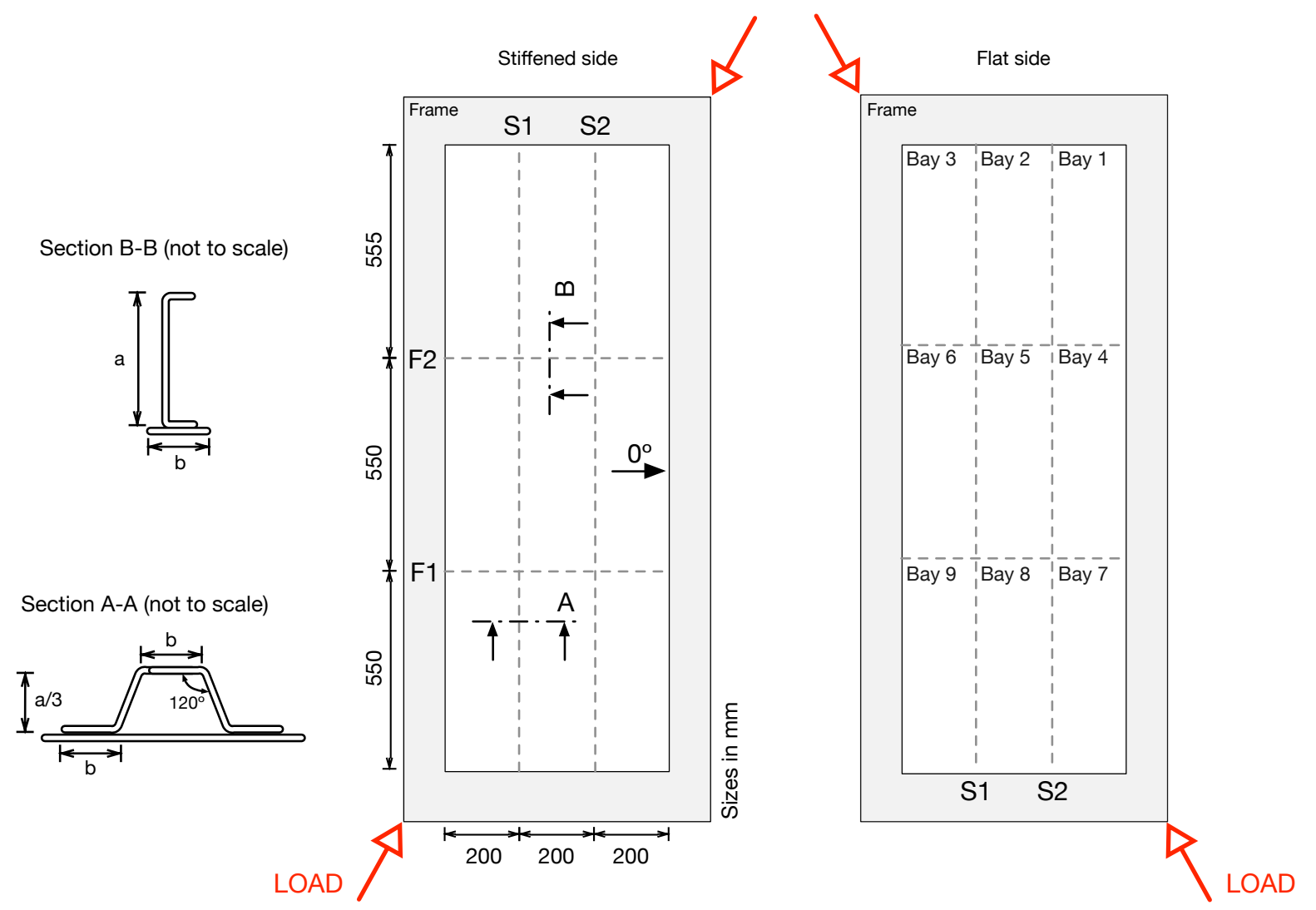

Figure 79. (Left) Dimensions of flat side panel with two metallic frames (F1 and F2), two omega stiffeners (S1 and S2), and the shear frame (dark grey) (Patricia F. Díaz-Maroto et al., 2018).

The main difference between panel 1 and 2 is the location of an acoustic layer between plies. For panel 1, two acoustic layers were incorporated alternating with constraint carbon plies. However, only one acoustic was incorporated.

The specimen was held in a rig so that the line connecting two of its four corners was perpendicular to the floor, also having the same direction as the applied load. A designed shear frame was manufactured to carry out this test, which mainly consists of interface plates, frame bar assemblies, and load bolt assemblies. Therefore, the specimen is loaded in 
shear applying an axial force, tension or compression, on the frame diagonal through the main load bolts assemblies.

\subsubsection{Testing Conditions}

The test was conducted on an MTS testing machine of $250 \mathrm{t}$ (see Figure 80). Strain monitoring for the buckling test was carried out through conventional back-to-back strain gauge rosettes and distributed fibre optic sensors. Additionally, a high-speed camera was placed in front of the panel. The shear frame can also be appreciated in Figure 80, which was designed to rig the panel into the testing machine and to constrain the out-of-plane displacement. However, it should be noted that an angle of $29^{\circ}$ exists between the diagonal of the frame and the diagonal of the panel. This fact may result in an out-of-plane (and thus, flexural) load on the specimen.

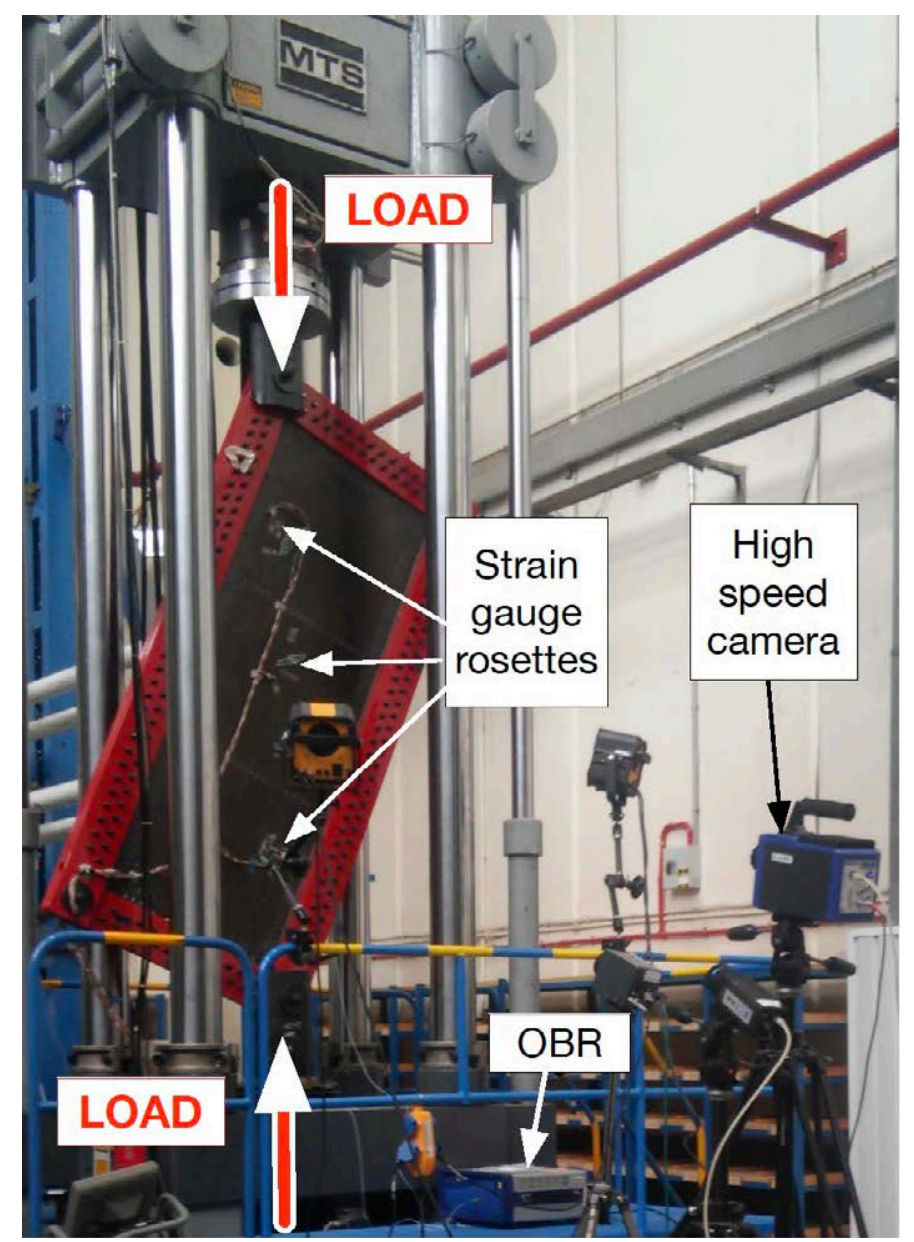

Figure 80. Support conditions, applied load for test specimen and sensors (Patricia F. DíazMaroto et al., 2018).

Furthermore, the panel mounted in this figure corresponds to the stiffened panel 1 . Here, the three back-to-back strain gauge rosettes can be seen in the diagonal panel, whereas the OBR system and high-speed camera are in front of the panel to monitor the entire test. 


\subsubsection{Sensor Instrumentation}

The distributed fibre optic sensing network as well as the strain gauge rosettes presented different configurations for each panel. Therefore, the description of the different sensing network is presented in detail in the following sections.

\subsubsection{Stiffened Panel 1}

For the stiffened panel 1, three pairs of back-to-back strain gauge rosettes were placed at Bay 3 (R1), Bay 5 (R2), and Bay 7 (R3) on both the stiffened and flat sides. In addition, they were located close to optical paths 2, 7, and 12 at each bay in the longitudinal direction for R1, R2, and R3, respectively. A single optical fibre was placed on the flat side panel made up of thirteen optical paths that enabled it to cover the entire surface in a crooked configuration. Owing to the accessibility of the panel configuration for this first test (already mounted), the fibre optic line was unable to be placed on the upper flat side (Bays 1 through 3). Only paths 1 and 2 are one-third longer than the rest of the paths.

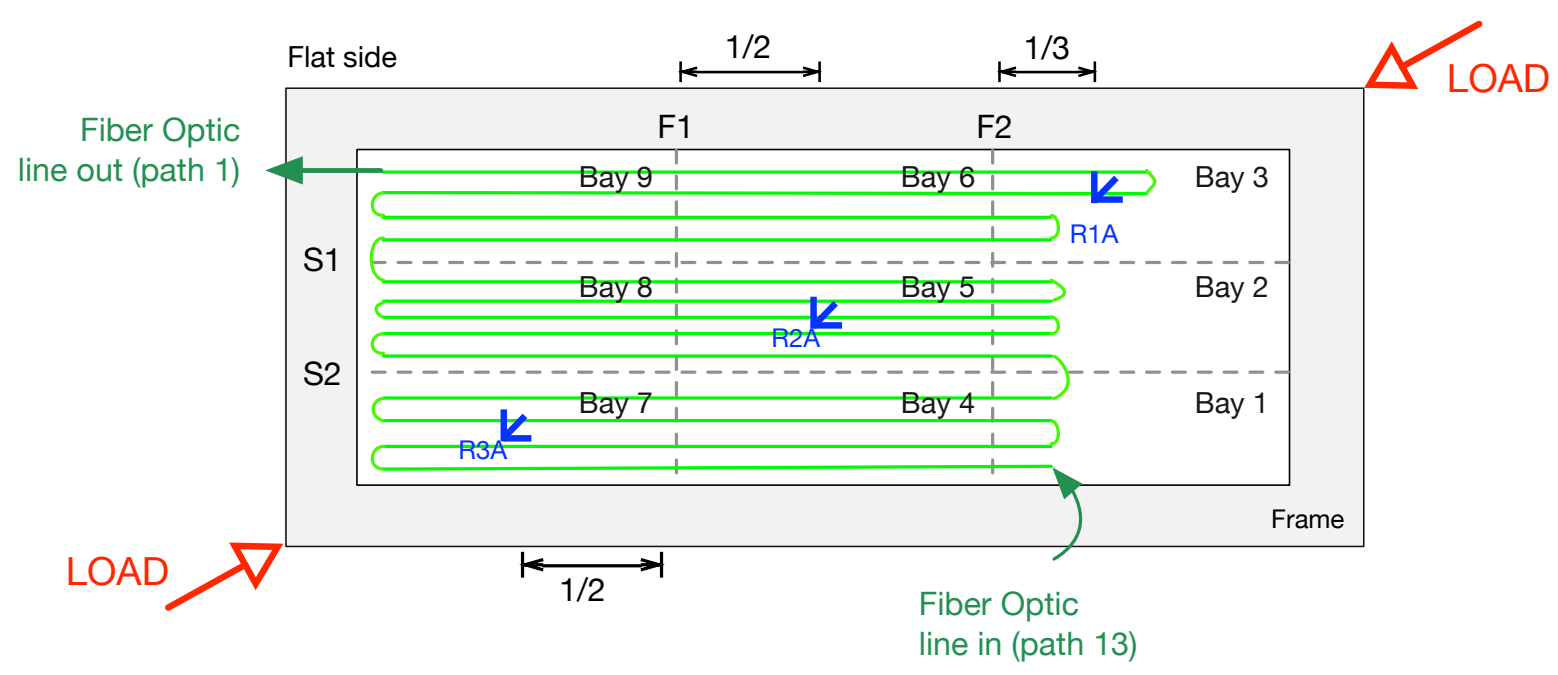

Figure 81. Detailed figure of the fibre optic sensing network and back-to-back strain gauges rosettes.

Figure 81 illustrates the relative position of the different sensors (fibre optic line and rosettes) over the stiffened panel side. It should be noted that the strain gauge rosettes were located close to the fibre optic line (about $5 \mathrm{~mm}$ ) but were unable to be placed in the same location.

\subsubsection{Stiffened Panel 2}

The specimen configuration and dimensions are the same as panel 1 (refer to Figure 79). However, regarding the sensor instrumentation, main differences for this panel are summarized here: 
- Two optical fibres are placed on the surface panel. Each fibre has 10 paths and 12 respectively. In this panel the whole flat side panel is covered with the 22 optical fibre paths.

- Eight back-to-back strain gauges were placed instead of the three pairs in the previous panel.

In Figure 82, the second stiffened panel is shown. Here, the two optical sensing networks can be differentiated. The ingress of the optical paths is located close to the long edges of the panel and the egress is located in the centre of the panel. The back-to-back rosettes are spread over the whole surface as can be observed in the figure. R1A, R2A and R3A were located on path 3 of the fibre optic line. Whereas R4A and R5A were located on path 12 and, R6, R7 and R8 on path 21. Furthermore, the wires of the different rosettes can be distinguished in the figure here below. This gives an idea of how cumbersome it can be when strain gauges are installed over the whole surface to obtain the same measurement points as a distributed measurement. Finally, damaged areas are marked with white shaded areas.

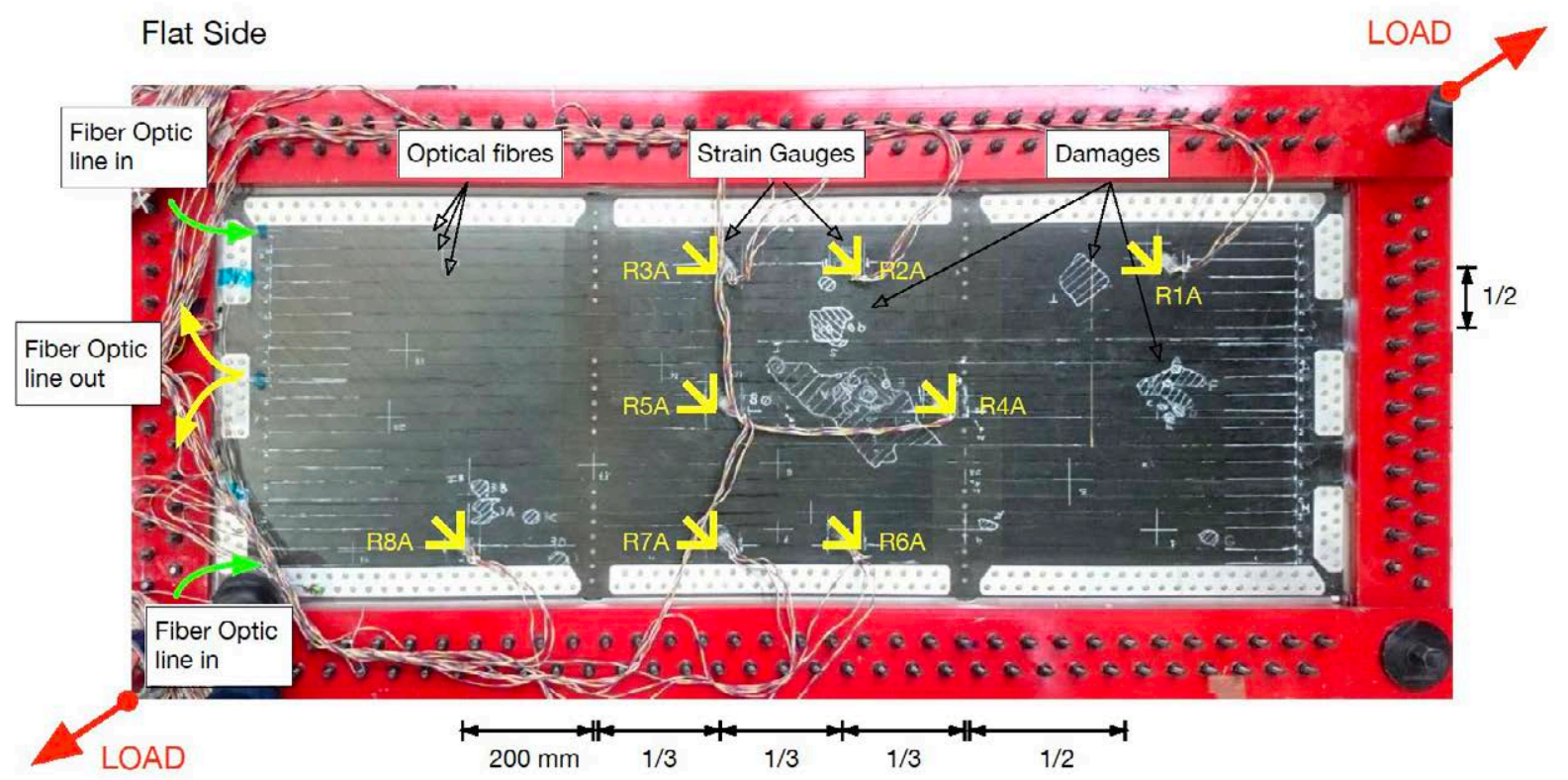

Figure 82. The second stiffened composite panel. The two fibre optic networks can be appreciated along with the strain gauges over the panel and the damage area.

As a result of the panel being much more instrumented than the previous one, Table 8 is an attempt to summarize the location of these sensors regarding the different structural parts. As a general rule, the back-to-back rosette " $a$ " is located on the flat side and " $b$ " is located on the stiffened side. 
Table 8. Sensors location for stiffened panel 2.

\begin{tabular}{|c|c|c|c|c|}
\hline Sensor & Area & Position & $\begin{array}{c}\text { Optical Fiber } \\
\text { Path }\end{array}$ & Side \\
\hline Thermal sensor & Omega 1-1 & Centered & - & Flat \\
\hline Back-to-back rosette $1 \mathrm{a} / \mathrm{b}$ & Bay 3 & Centre & 3 & Flat/ Stiffened \\
\hline Back-to-back rosette $2 \mathrm{a} / \mathrm{b}$ & Bay 6 & Centre $1 / 3$ up & 3 & Flat/ Stiffened \\
\hline Back-to-back rosette $3 \mathrm{a} / \mathrm{b}$ & Bay 6 & Centre $1 / 3$ down & 3 & Flat/ Stiffened \\
\hline Back-to-back rosette $4 \mathrm{a} / \mathrm{b}$ & Bay 5 & Centre up & 12 & Flat/ Stiffened \\
\hline Back-to-back rosette $5 \mathrm{a} / \mathrm{b}$ & Bay 5 & Centre $1 / 3$ down & 12 & Flat/ Stiffened \\
\hline Back-to-back rosette $6 \mathrm{a} / \mathrm{b}$ & Bay 4 & Centre $1 / 3$ up & 21 & Flat/ Stiffened \\
\hline Back-to-back rosette $7 \mathrm{a} / \mathrm{b}$ & Bay 4 & Centre $1 / 3$ down & 21 & Flat/ Stiffened \\
\hline Back-to-back rosette $8 \mathrm{a} / \mathrm{b}$ & Bay 7 & Centered middle up & 21 & Flat/ Stiffened \\
\hline
\end{tabular}

Figure 83 is a detailed picture of the optical fibre paths when they are located at the same position as the rosettes. When this occurs, the fibre is placed over the rosettes without bonding in order to avoid the fibre breakage. This operation for fibre optic line installation is mandatory since the back-to-back strain gauge rosette will not able to measure if it is not bonded to the panel surface. However, in the case of the optical fibre it only fails at some measurement points around the rosette location.

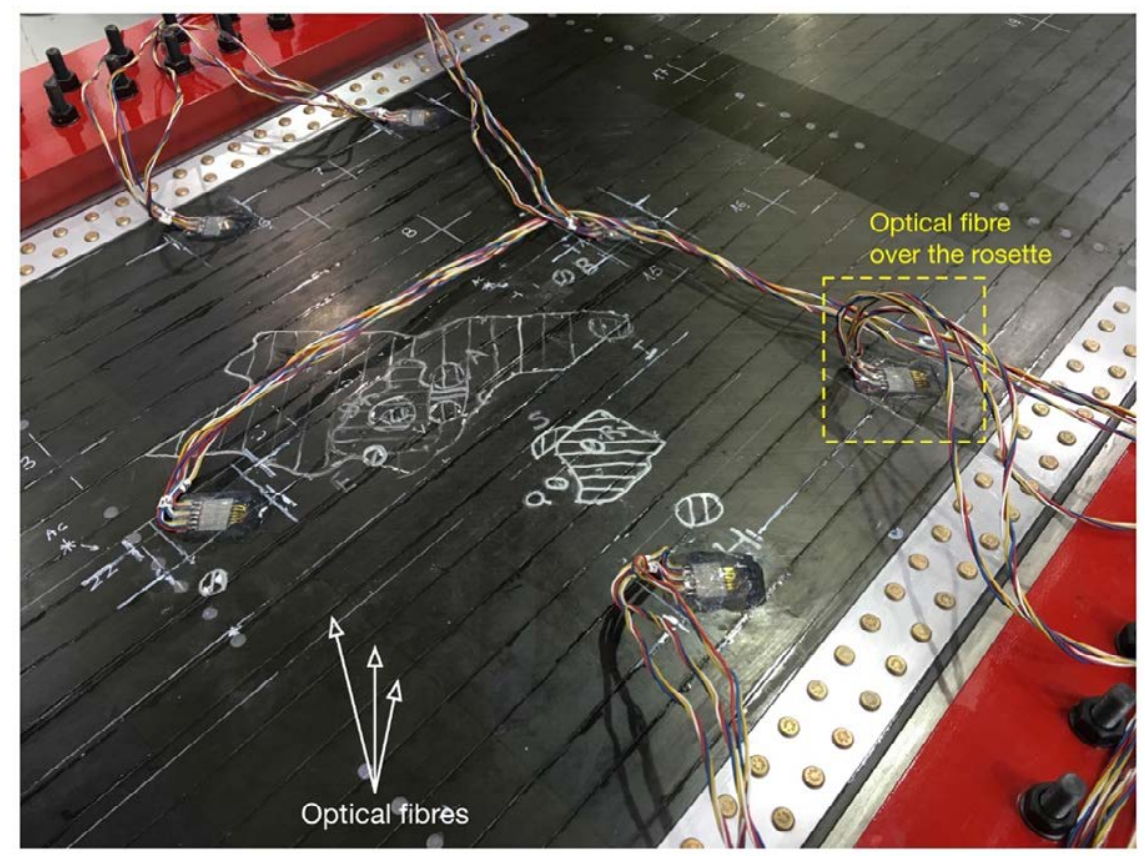

Figure 83. Detailed figure of the optical fibre regarding the rosettes location. 
Furthermore, the optical fibres can be distinguished on the flat side. The separation between the two consecutive paths was of $20 \mathrm{~mm}$.

\subsubsection{Test Sequence}

For both stiffened panels the test sequence was a pointed out in the following list:

- Zero inspection.

- Impact test.

- Stiffened panel assembly in the test frame.

- Fatigue test and damage growth.

- Buckling test.

The load for the static test corresponds to a typical cruise flight. The following hypotheses were assumed:

- $25 \%$ of post-buckling capacity (UL $=1.25 \times$ Buckling Load).

- $\mathrm{UL}=\mathrm{LL} \times 1.5$.

- Typical cruise flight load $=\frac{\text { flight load }}{\text { limit load }}=\frac{1}{1.33}=0.75$

Then, based on the theoretical buckling load obtained (see Table 11) the first stiffened panel was tested under $1.25 \times$ LL, and the second stiffened panel under UL. Further description of the test sequence followed by each panel is detailed in the following sections.

\subsubsection{Stiffened Panel 1}

Firstly, the stiffened panel 1 was submitted to the zero inspection to ensure every component was correct and the structure had no damage. For this objective, NDT was carried out.

After the impact test was performed, the panel was assembled in the test frame to introduce two fatigue lives (180 k cycles). Due to the most critical load for this type of panel configuration is under compressive load, the first panel was only tested to on this load (no tensile load was applied to this panel). Then, for the buckling test, a diagonal compressive load was introduced to the panel linearly from 20 to $160 \mathrm{kN}$ at load step increments of $20 \mathrm{kN}$, and from 160 to $195 \mathrm{kN}$ by load step increments of $5 \mathrm{kN}$. The sequence for this panel is resumed in Table 9. 
Table 9. Test sequence for stiffened panel 1.

\begin{tabular}{|c|c|c|c|c|c|}
\hline Panel & Sequence & $\begin{array}{c}\text { Test } \\
\text { Type }\end{array}$ & $\begin{array}{c}\text { Cycles } \\
{[\mathrm{k}]}\end{array}$ & $\begin{array}{c}\text { Compressive Test } \\
{[\mathrm{kN}]}\end{array}$ & $\begin{array}{c}\text { Tensile Test } \\
{[\mathrm{kN}]}\end{array}$ \\
\hline 1 & 0 & Zero inspection & 0 & - & - \\
\hline & 1 & Impact test & 0 & - & - \\
\hline & 2 & Fatigue & 179650 & $-180 \mathrm{kN}$ & $+180 \mathrm{kN}$ \\
\hline & 3 & Fatigue & 179800 & $-180 \mathrm{kN}$ & $+180 \mathrm{kN}$ \\
\hline & 4 & Fatigue & 180 & $-180 \mathrm{kN}$ & $+180 \mathrm{kN}$ \\
\hline & 5 & Buckling & 180 & $-195 \mathrm{kN}$ & - \\
\hline
\end{tabular}

\subsubsection{Stiffened Panel 2}

In Table 10, the sequence of the different tests carried out for this second stiffened panel is presented. For this panel, also two fatigue lives were tested (180000 cycles) and a panel was loaded at each half-life (i.e. $45 \mathrm{k}, 90 \mathrm{k}$ and $135 \mathrm{k}$ cycles). The aim of the distributed measurement acquired during the two fatigue lives is to analysis the increase of the damages of the structure. Due to the damages of the second panel being more critical, the selected load was lower than those used for the first panel. Finally, the buckling test was carried out up to $-235 \mathrm{kN}$ of compressive load and then, up to $+300 \mathrm{kN}$ of tensile test.

Table 10. Test sequence for stiffened panel 2.

\begin{tabular}{|c|c|c|c|c|c|}
\hline Panel & Sequence & $\begin{array}{c}\text { Test } \\
\text { Type }\end{array}$ & $\begin{array}{c}\text { Cycles } \\
{[\mathrm{k}]}\end{array}$ & $\begin{array}{c}\text { Compressive Test } \\
{[\mathrm{kN}]}\end{array}$ & $\begin{array}{c}\text { Tensile Test } \\
{[\mathrm{kN}]}\end{array}$ \\
\hline 2 & 0 & Zero inspection & 0 & - & - \\
\hline 1 & Impact test & 0 & - & - \\
\hline 2 & Fatigue & 0 & -180 & +180 \\
\hline & 3 & Fatigue & 45 & -120 & +120 \\
\hline 4 & Fatigue & 90 & -120 & +120 \\
\hline 5 & Fatigue & 135 & -120 & +120 \\
\hline 6 & Fatigue & 180 & -120 & +120 \\
\hline 7 & Buckling & 180 & -235 & +300 \\
\hline
\end{tabular}




\subsection{Theoretical Buckling Load}

In order to estimate the buckling load, a FEM model of the stiffened panel was performed. The buckling test under compressive load was calculated by Nastran 105 solution. Therefore, the critical buckling load was obtained by multiplying the FEM load applied by the first eigenvalue obtained. Table 11 is a summary of the eigenvalues of the first and second modes obtained for the compressive buckling test.

Table 11. Buckling critical load for tensile and compressive test.

\begin{tabular}{|c|c|c|c|c|}
\hline Test & $\begin{array}{c}\text { FEM Load } \\
{[\mathrm{N}]}\end{array}$ & Mode & Eigenvalue & $\begin{array}{c}\text { Buckling Load } \\
{[\mathrm{N}]}\end{array}$ \\
\hline Compressive & -143313 & 1 & 1.2916 & -185103 \\
\hline Compressive & -143313 & 2 & 1.3013 & -186493 \\
\hline
\end{tabular}

As can be seen in Figure 84, the buckling appearance under a compressive load of $-143.3 \mathrm{kN}$ prompts a first buckling mode at bay 7 under $-185.1 \mathrm{kN}$. It must be pointed out that the first and second eigenvalues under compressive load are roughly identical (difference $<1 \%$ ), and therefore, it can be considered that buckling can appear at both bays 3 and 7. Figure 84 shows the buckling appearance for first and second mode under a compressive load of -143.3 $\mathrm{kN}$.
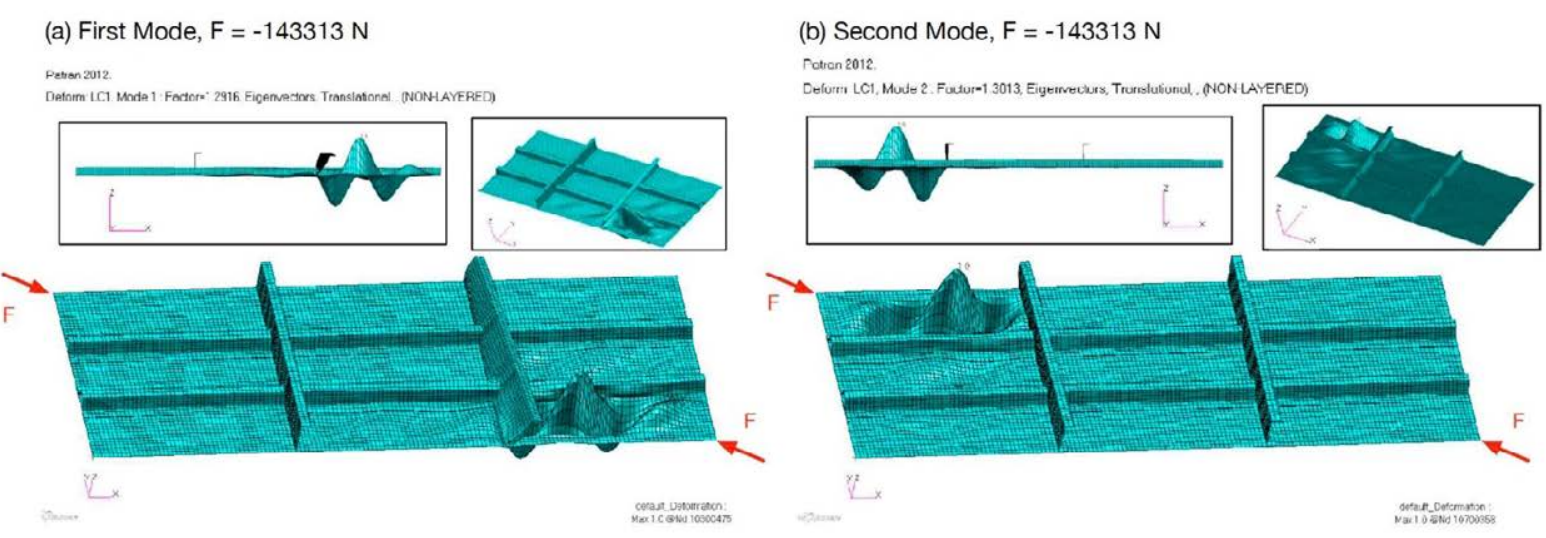

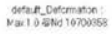

Figure 84. Buckling appearance under compressive load. (a) First and (b) second mode.

\subsection{Temperature Compensation for Distributed Measurement}

As described in the distributed measurement section, in order to discriminate the temperature change from the strain measurements an additional temperature sensor is needed. For these tests, a thermocouple was used to measure the room temperature.

First, the distributed measurement is obtained as shown in Equation 6-1, i.e. the OBR measurement is the combination of these two terms 


$$
\Delta \varepsilon=\frac{1}{K_{\varepsilon}}\left(-\frac{\Delta v}{v}-\Delta T \cdot K_{T}\right)=-\frac{1}{v K_{\varepsilon}} \Delta v-\frac{K_{T}}{K_{\varepsilon}} \Delta T=-\frac{\bar{\lambda}}{c K_{\varepsilon}} \Delta v-\frac{K_{T}}{K_{\varepsilon}} \Delta T
$$

If the temperature remains constant, the measurement will refer only to the strain variation. The strain and temperature coefficients can be obtained directly from the OBR measurement. In this test the strain and temperature coefficient are obtained as shown in Equation 6-2 and Equation 6-3.

$$
\begin{gathered}
\text { Strain coefficient }=S_{\text {coeff }}=-\frac{\bar{\lambda}}{c K_{\varepsilon}}=-6.6680002^{\mu \varepsilon} / \mathrm{GHz} \\
\text { Temperature coefficient }=T_{\text {coeff }}=-\frac{\bar{\lambda}}{c K_{T}}=-0.801388^{\circ} \mathrm{C} / \mathrm{GHz}
\end{gathered}
$$

Hence, the compensation factor can be obtained by dividing the strain coefficient between the temperature coefficient (see Figure 85). In general, the temperature changes recorded through this experiment oscillate between $0.1-0.5{ }^{\circ} \mathrm{C}$, which produce no significant strain changes in the order of the microstrains. It must be pointed out that the transfer of the temperature changes is considered perfectly transmitted to the optical fibres.

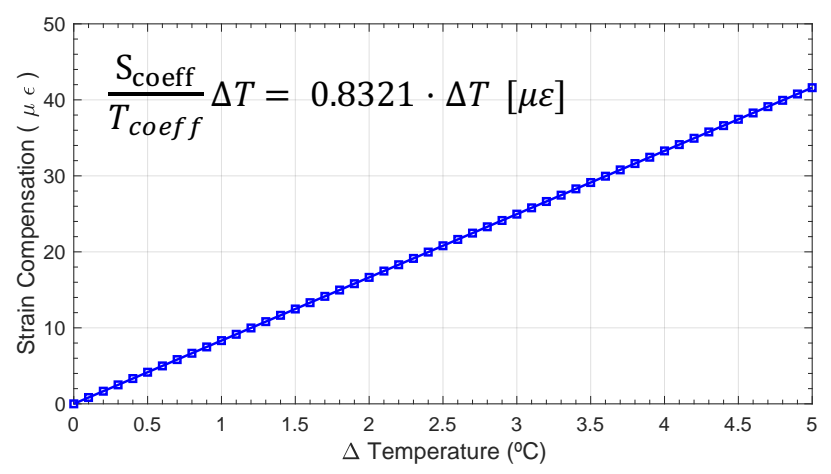

Figure 85. Strain compensation by temperature increment effect.

All distributed measurements here presented were temperature compensated as described in this section.

\subsection{Analysis of Stiffened Panel 1}

The results of the different tests carried out for this first stiffened panel is presented in the following sections.

\subsubsection{Impact Test}

Four impacts were performed on the test specimen through the free-falling method according to AITM 1-0010. They were carried out with a $15.8 \mathrm{~mm}$ diameter penetrator. The impacted areas are shown in Figure 86. No indentation depth was observed regarding these 
four impacts. It must be noted that impact 2 is out of the sensing area cover by the distributed fibre optic line, as well as the impact 4, which is located in the middle of the stiffeners foot. However, the back-to-back strain gauge rosette is located near impact 2, and the strain readings can be analysed from these measurements.

A summary of all impacts that can be analysed by the fibre optic line are specified in Table 12. After each impact, the affected area was inspected by ultrasonic manual pulse echo according to NTM 51-10-09. In addition, after the impacts caused by the free-falling method, additional impacts were performed by means of compression air mobile impact device (CAMID) also applying a $15.8 \mathrm{~mm}$ diameter penetrator. After the CAMID test and due to the higher impact energy, slight indentation can be appreciated at the location of the impact.

All impacts were carried out on the flat side prior to installing the optical fibre line in order to avoid the breakage of the fibre.

Table 12. Specification of impacts for stiffened panel 1.

\begin{tabular}{|c|c|c|c|c|c|}
\hline Impact & Zone & $\begin{array}{l}\text { Impact } \\
\text { Side }\end{array}$ & $\begin{array}{c}\text { Impact } \\
\text { Energies [J] }\end{array}$ & Impactor & $\begin{array}{c}\text { Damage } \\
\text { Characterization }\end{array}$ \\
\hline \multirow[t]{2}{*}{1} & \multirow[t]{2}{*}{ Bay 5} & \multirow[t]{2}{*}{ Flat } & 5,10 & $\begin{array}{l}\text { Gravity } \\
\text { Impactor }\end{array}$ & $\begin{array}{l}\text { Multiple delaminations at } \\
\text { different depths }\end{array}$ \\
\hline & & & 25 & CAMID & Delamination at last layer \\
\hline \multirow[t]{2}{*}{2} & \multirow[t]{2}{*}{ Bay 3} & \multirow[t]{2}{*}{ Flat } & 5,10 & $\begin{array}{l}\text { Gravity } \\
\text { Impactor }\end{array}$ & Debonding at stringer foot \\
\hline & & & 35 & CAMID & Multiple delaminations \\
\hline \multirow[t]{2}{*}{3} & \multirow[t]{2}{*}{$\begin{array}{c}\text { Bay } 7 \text { / } \\
\text { Stringer S2 }\end{array}$} & \multirow[t]{2}{*}{ Flat } & $5,10,15$ & $\begin{array}{l}\text { Gravity } \\
\text { Impactor }\end{array}$ & $\begin{array}{l}\text { Multiple delaminations at } \\
\text { different depths, debonding at } \\
\text { stringer foot }\end{array}$ \\
\hline & & & 35 & CAMID & Debonding at stringer foot \\
\hline \multirow[t]{2}{*}{4} & \multirow[t]{2}{*}{ Stringer S2 } & \multirow[t]{2}{*}{ Flat } & 5,10 & $\begin{array}{l}\text { Gravity } \\
\text { Impactor }\end{array}$ & $\begin{array}{l}\text { Multiple delaminations at } \\
\text { different depths }\end{array}$ \\
\hline & & & 20 & CAMID & Delamination at last layer \\
\hline
\end{tabular}

A detailed picture of these impacts can be appreciated in Figure 87, as well as the impact energy and the dimensions of the delaminated area. Since impact 2 and impact 4 are located far from the fibre optic line delamination, the damage cannot be analysed as explained above. Nevertheless, the impact 3 is located over fibre optic path 10 and the impact 1 on path 8 and slightly on path 7 as well. 


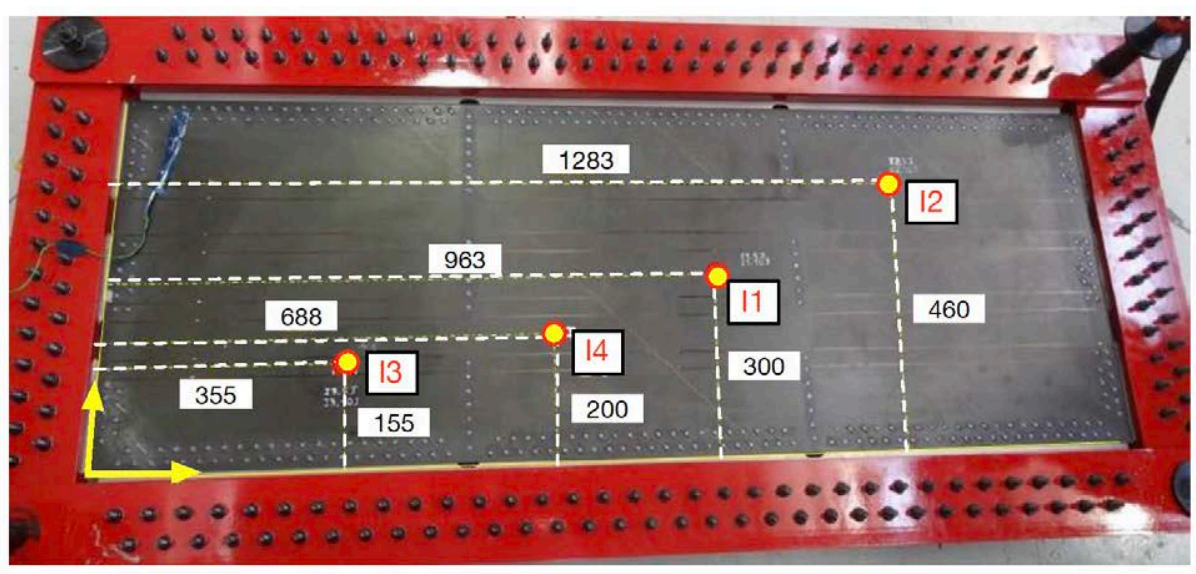

Figure 86. Impact locations on the stiffened panel 1. Start and end lines of the fibre optic paths.
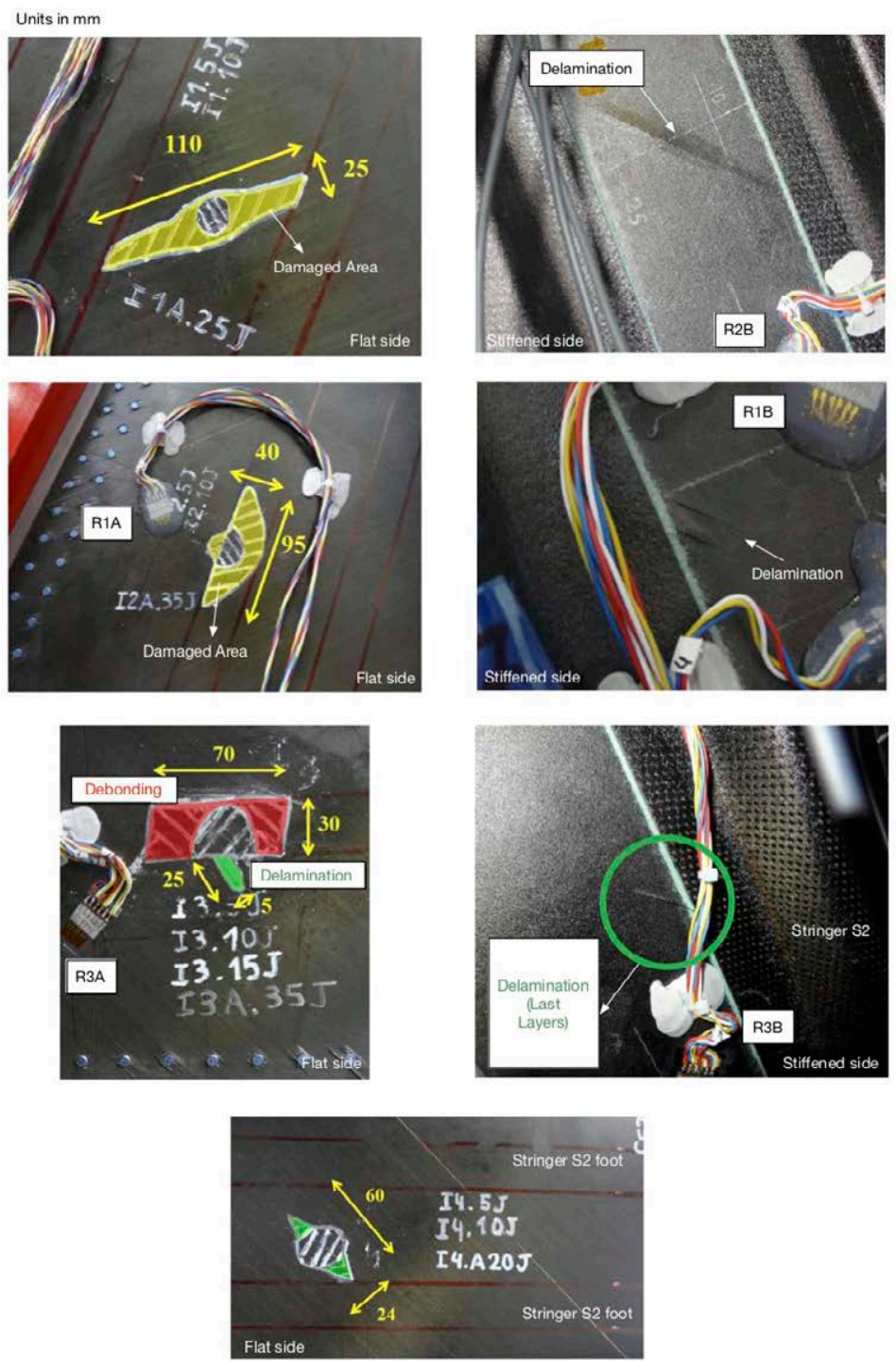

Figure 87. All impacts, from impact 1 (top) to 4 (bottom). The damaged area is expressed in millimetres. 


\subsubsection{Fatigue Test and Damage Growth}

Prior to performing the buckling test, a fatigue test was completed as explained in 6.2.4.1. The two fatigue panel lives correspond to 180000 cycles, and the distributed measurement was acquired before finishing its second fatigue life. The aim of this test was to study the growth of the delamination caused by the impact test. Three distributed strain measurements were acquired at 179650, 179800 and 180000 cycles. The load of the fatigue test was $\pm 180 \mathrm{kN}$. Figure 88 shows the residual strain after each fatigue test load test. The acquired signal seemed to be noisy due to the strain level being so small. However, the different paths can be distinguished in the whole distributed measurement with a mean of residual strains of $15 \mu \varepsilon$.

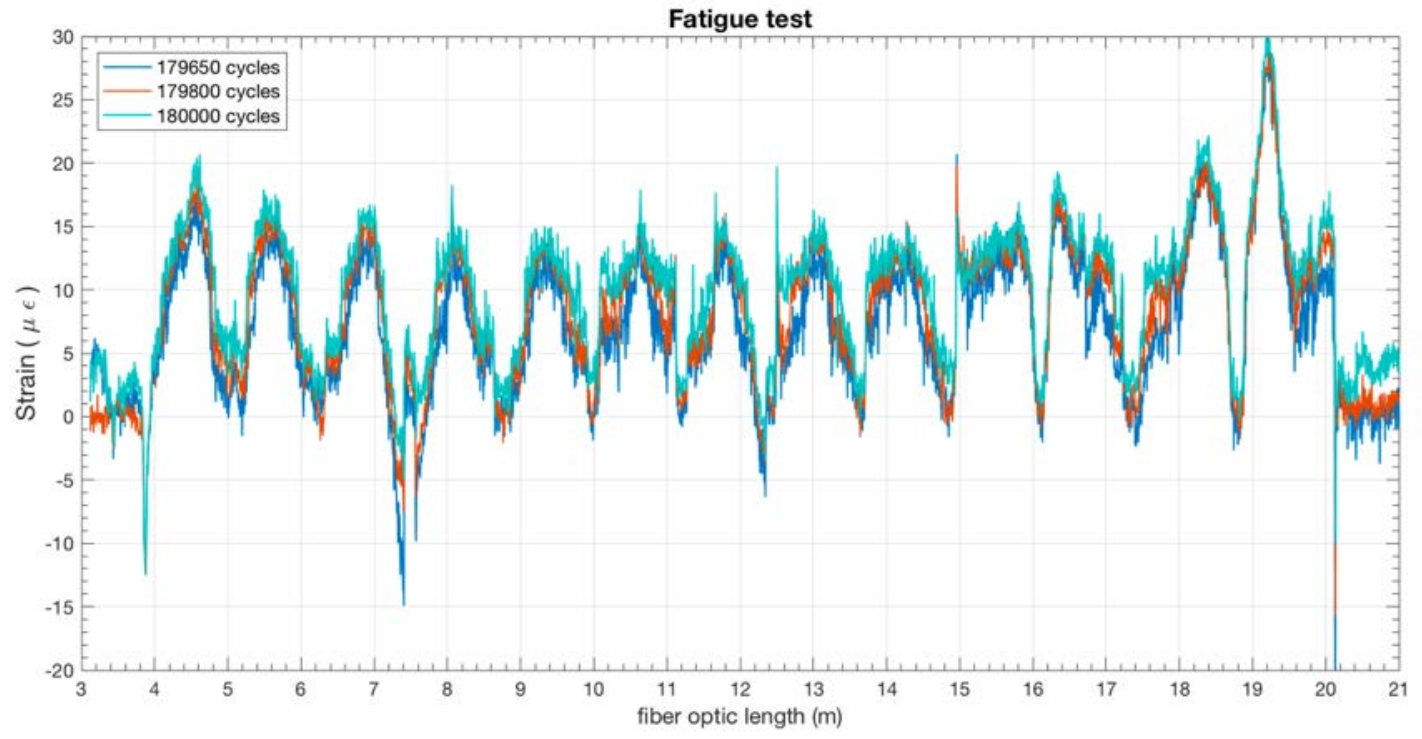

Figure 88. Fatigue Test

Figure 89 illustrates the impact 1 location over the length of paths 7 and 8 . A slight level of residual strain can be observed, being accumulated in the nearby area of the impact for both paths. However, it was not enough to grow the delamination of the damage.
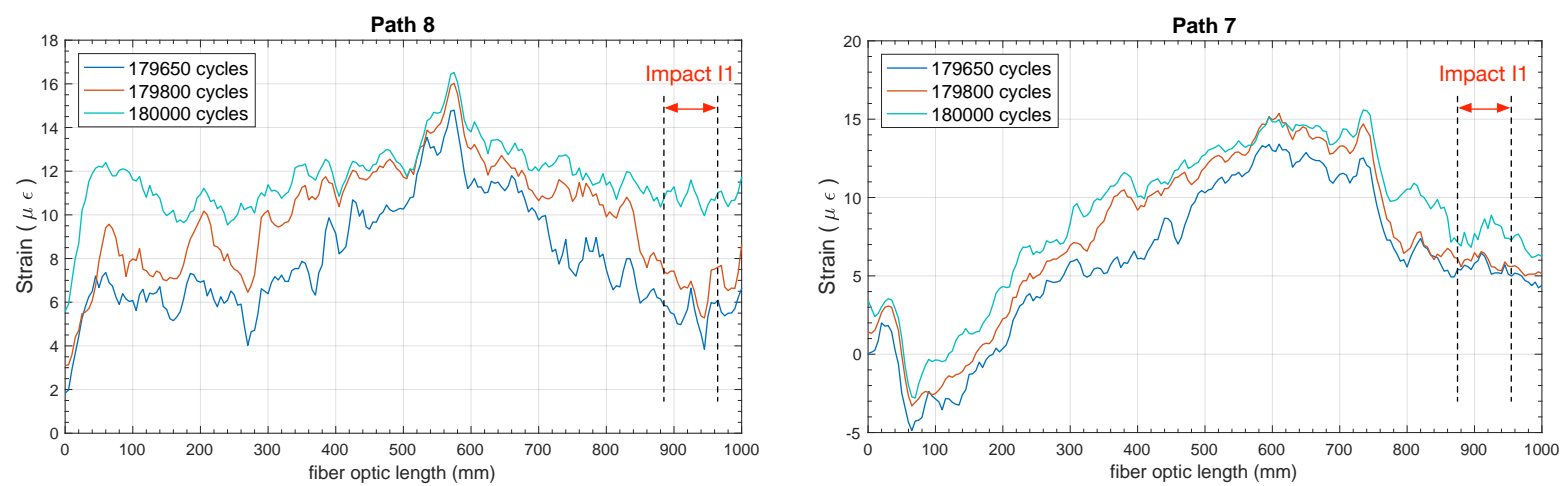

Figure 89. Residual strains obtained from the distributed measurement around the impact 1. 
In Figure 90, impact 3 is located over path 10. Just as in the case of impact I1, the residual strain level is still very low. A slightly difference of strain can be appreciated in the area close to the impact I3. The growth of the damage cannot be assured since the residual strain level is not significant enough.

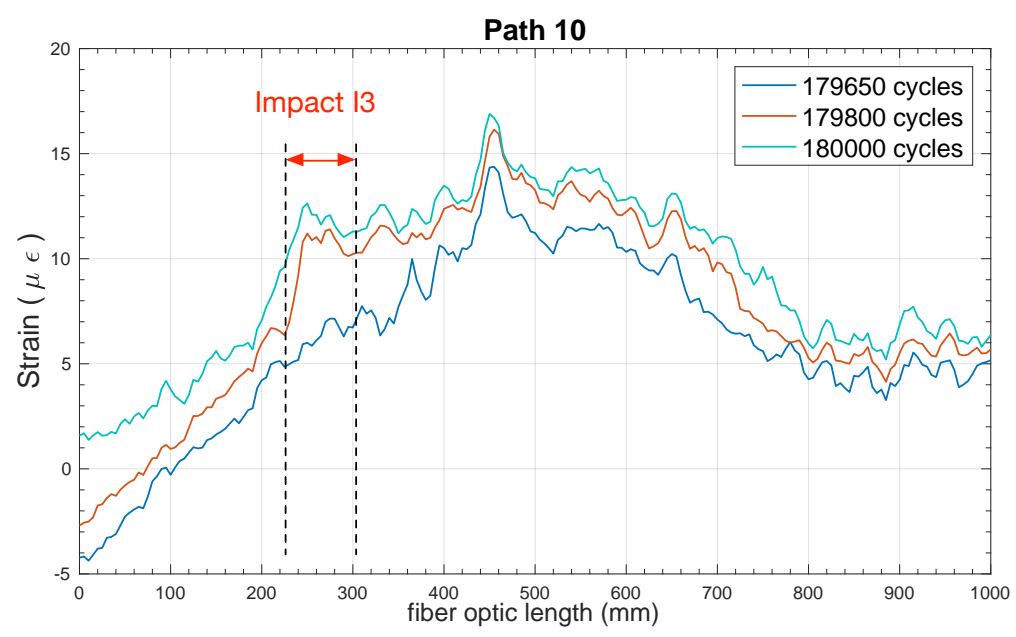

Figure 90. Residual strains obtained from the distributed measurements around the impact 3.

The growth of the damages by means of the rosettes strain readings in the buckling test is analysed in section 6.5.3.1.

\subsubsection{Buckling Analysis}

In the following sections the analysis of the buckling onset will be analysed by means of the strain gauge rosettes strain readings, the distributed fibre optic network and high-speed camera. The critical buckling load is identified and compared with the predicted theoretical buckling load.

\subsubsection{Strain Gauge Rosettes Results}

Strain gauge rosettes measured throughout the buckling test. Because the panel was under shear loads, the results of the rosettes was expressed in terms of the shear strains as shown in Equation 6-4.

$$
\gamma_{x y}=2 \varepsilon_{b}-\varepsilon_{a}-\varepsilon_{c}
$$

Here, $\varepsilon_{a}, \varepsilon_{b}$, and $\varepsilon_{c}$ are the axial strains at $0^{\circ}$ (fibre optic direction), 45으, and $90^{\circ}$, respectively, for the flat panel side.

In rosette R1B, it can be observed that at a load level between -140 and $-160 \mathrm{kN}$, the strain readout started to show non-linear behaviour until the load buckling appearance at $-185 \mathrm{kN}$ (see Figure 91a). Nevertheless, R2A and R2B have a perfect linear behaviour against the load 
(see Figure 91b), and R3 starts to become slightly non-linear from $140 \mathrm{kN}$ because it was located close to the buckling area (see Figure 91c).

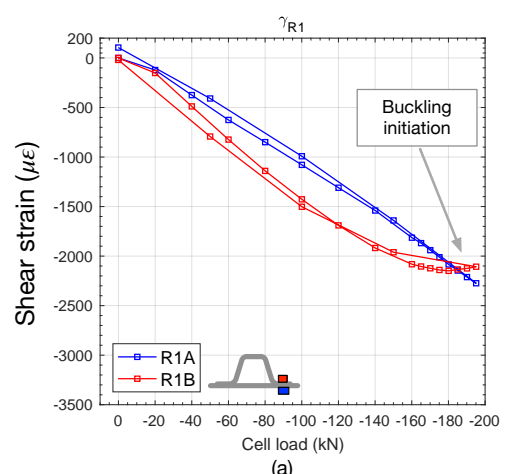

(a)

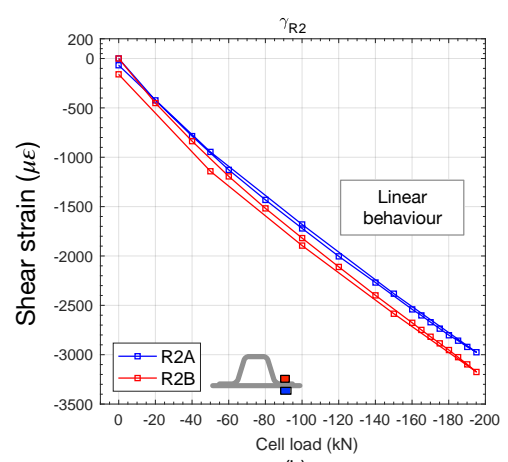

(b)

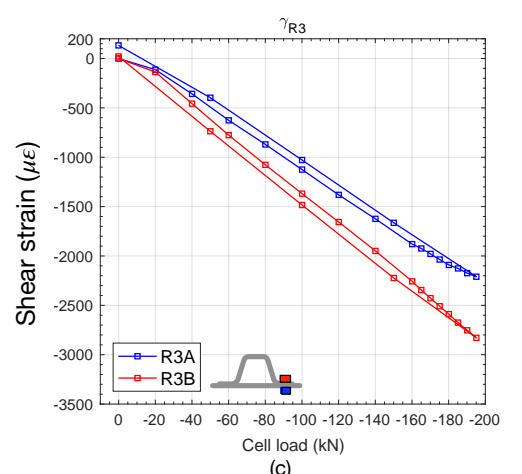

(c)

Figure 91. Load-strain curves from rosettes (a) R1 (b) R2, and R3 (c) (Patricia F. Díaz-Maroto et al., 2018).

Furthermore, a different slope in R1 and R3 can be observed between the stiffened and the flat sides. This behaviour is caused by the non-alignment between the frame and panel diagonals (2.9 $9^{\circ}$ of difference). As expected, the slope of R2 strain measurements for both sides was identical since it was located at the centre of the bay at the diagonals intersection. Despite the frame constraining the panel skin to avoid this effect, it becomes more evident under higher compressive loads.

The presence of the damaged area can also be observed at the strain reading of each rosette. Some significant residual strains were recorded on the flat side of the panel close in R1A, which was located close to impact 2 . The growth of these delaminations after the buckling test caused the release of significant strains on the flat side. Despite there being a delamination that could be appreciated at the stiffened side, it was located at the stringer foot where the delamination was stopped.

Regarding the residual strains of R2, the maximum value was recorded at a $45^{\circ}$ angle. As can be seen in Figure 87, the delaminated area for the impact 1 was also along this angle. The delamination was appreciated at the stiffened side, i.e. R2B, in the last layer. Therefore, after the buckling test it can be affirmed that this delamination growth.

Furthermore, significant strains were released on the flat side close to R3A where impact 3 was located. The high residual strain value recorded at this rosette is mostly due the debonding of the surrounding impacted area. 
Table 13. Residual strains of strain gauge rosettes after buckling test.

\begin{tabular}{c|c|c|c|c|c|c|}
\hline \multirow{2}{*}{ Strain } & \multicolumn{2}{|c|}{ Rosette 1 } & \multicolumn{2}{c|}{ Rosette 2 } & \multicolumn{2}{c|}{ Rosette 3 } \\
\hline Reading & R1A & R1B & R2A & R2B & R3A & R3B \\
{$[\mu \varepsilon]$} & {$[\mu \varepsilon]$} & {$[\mu \varepsilon]$} & {$[\mu \varepsilon]$} & {$[\mu \varepsilon]$} & {$[\mu \varepsilon]$} \\
\hline $0 \cong$ & 4 & -20 & -9 & -29 & -1 & -56 \\
\hline $45 \varrho$ & 49 & -25 & -16 & -77 & 49 & -32 \\
\hline $90 \varrho$ & -11 & -10 & 45 & 35 & -34 & -29 \\
\hline$\gamma_{x y}$ & 105 & -20 & -68 & -160 & 133 & 21 \\
\hline
\end{tabular}

The $0^{-}$axis of the rosette strain gauge provides information in agreement with the distributed optical fibre measurement (see Figure 92-upper). It can be observed that R1A-a was linearly loaded up to $-160 \mathrm{kN}$, when it suddenly collapsed as the load was increased. Similar behaviour is shown at R3A-a but at an earlier load of $-140 \mathrm{kN}$. This behaviour indicates local buckling and non-linearity close to the rosette location. Nevertheless, R2A-a, located at the centre of the stiffened panel, remained linear until reaching $-195 \mathrm{kN}$.

The $45^{\circ}$ axis of the rosette strain gauge recorded the maximum strain level as expected (almost aligned with the load direction). Same behaviour as explained above is obtained in this direction.
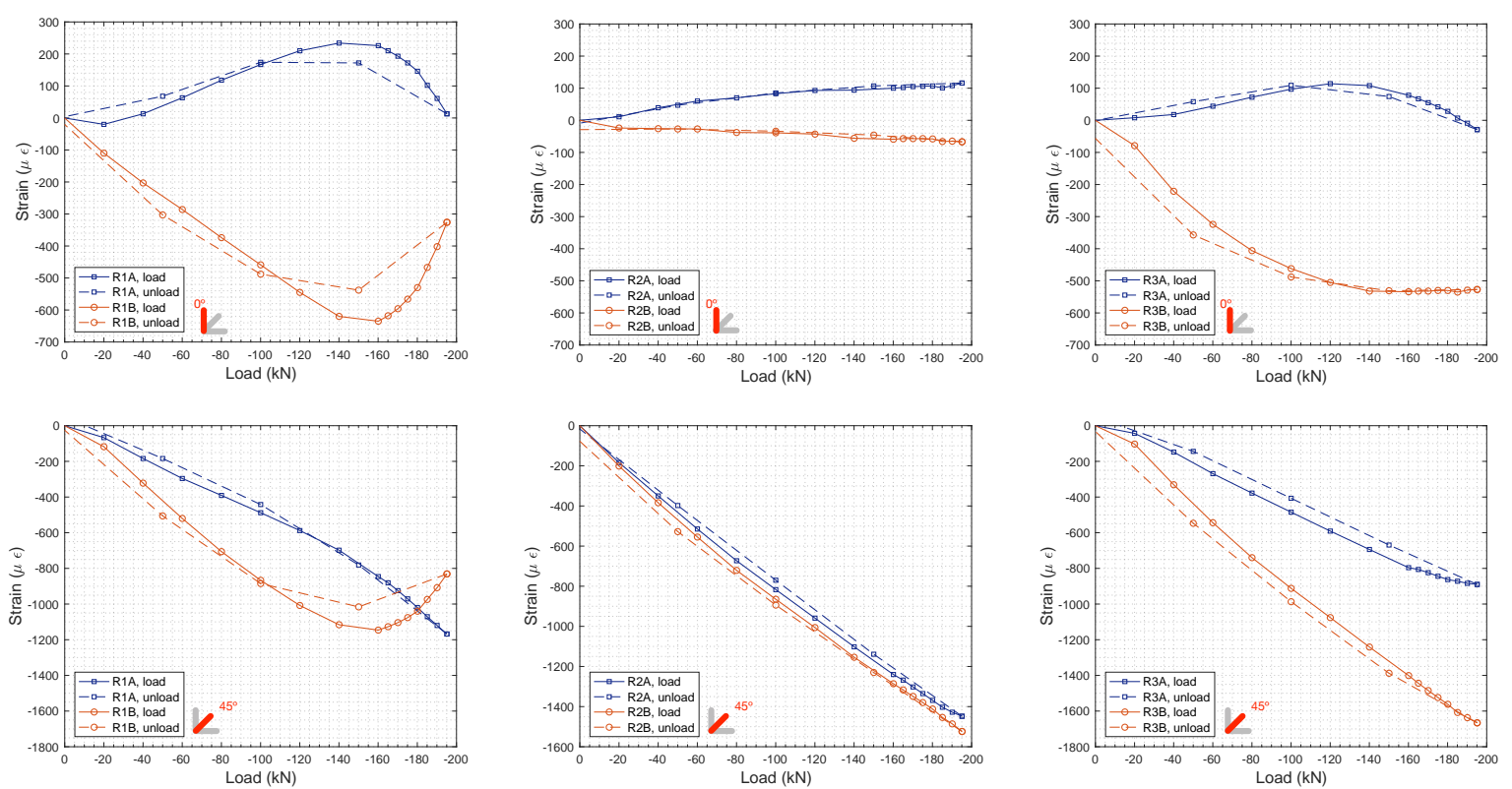

Figure 92. Load-strain curves for the $0^{\circ}$ and $45^{\circ}$ of the rosettes R1, R2 and R3.

\subsubsection{Distributed Strain Measurement Results}

Distributed strain measurements through the OBR system were recorded at each load step. From the single fibre optic line, the total length of which was roughly $20 \mathrm{~m}$, different paths 
were extracted to keep the valuable strain data for a buckling analysis. The distributed measurements presented were conducted using a gauge length of $5 \mathrm{~mm}$, with $5 \mathrm{~mm}$ spacing between consecutive sensors to avoid an overlap.

As the room temperature was kept constant during the test, the distributed measurement was not affected by temperature variations, measuring only the strain changes. Figure 93 shows all the distributed strain measurements for different load steps recorded during the test, including loops and fibre connectors. It is observed that the strain level is lower in the middle of the distributed measurement (paths 9 through 6), where the centre bays were located between stiffeners, and is higher at the boundaries, where the buckling was initiated. These paths are thoroughly analysed below.

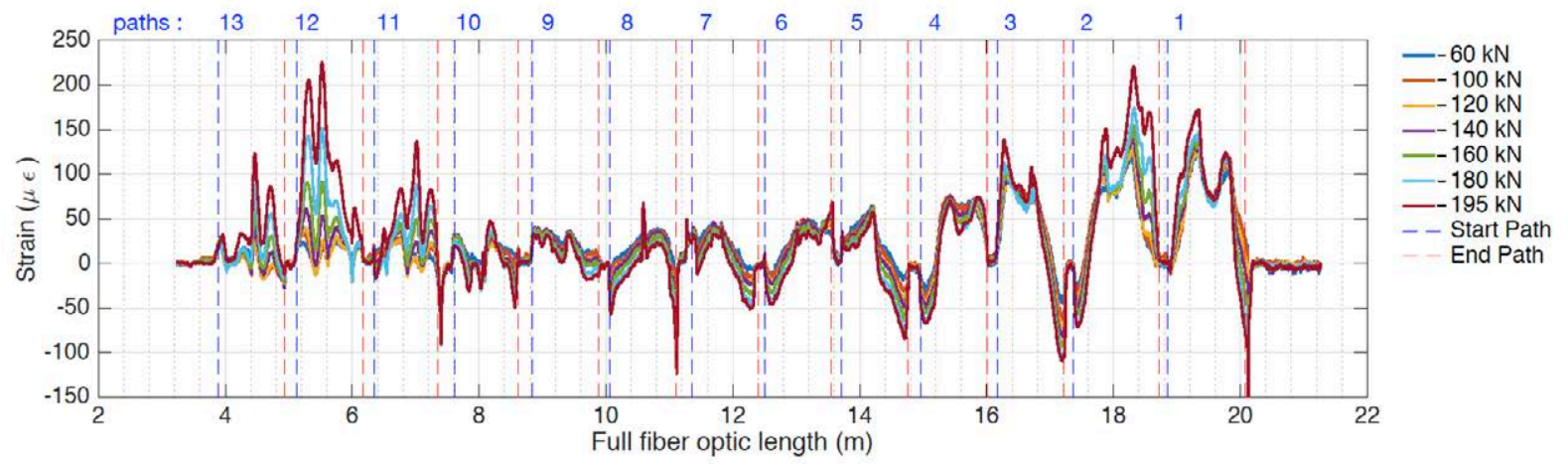

Figure 93. Full distributed strain measurement of the fibre optic line (Patricia F. Díaz-Maroto et al., 2018).

Figure 94 shows the full distributed measurement placed on the panel's flat side in 3D. From these measurements the distributed measurement map can be reconstructed.

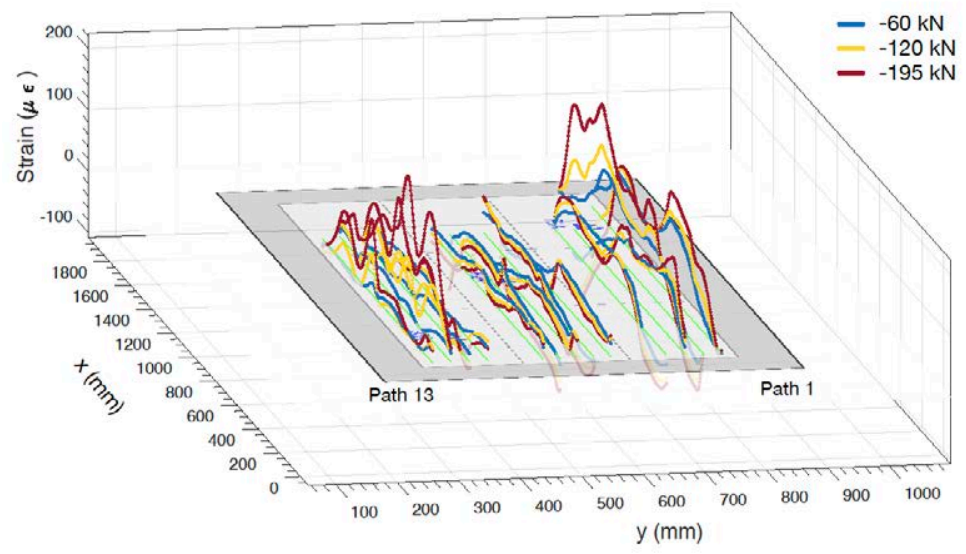

Figure 94. All distributed measurement paths in 3D for $-60 \mathrm{kN},-120 \mathrm{kN}$ and $-195 \mathrm{kN}$ (Patricia F. Díaz-Maroto et al., 2018).

Figure 95 illustrates distributed measurements on paths 10,11, 12, and 13, which were located at Bays 4 and 7 . At the same buckling load detected by strain gauge rosette readings, at a compressive load of $-185 \mathrm{kN}$, some locations with a non-linear behaviour (e.g. dashed 
red squares) are observed. It can also be pointed out that Path 10 has a lower strain owing to the stiffness of the stringer foot, but its behaviour remains similar to the other paths.
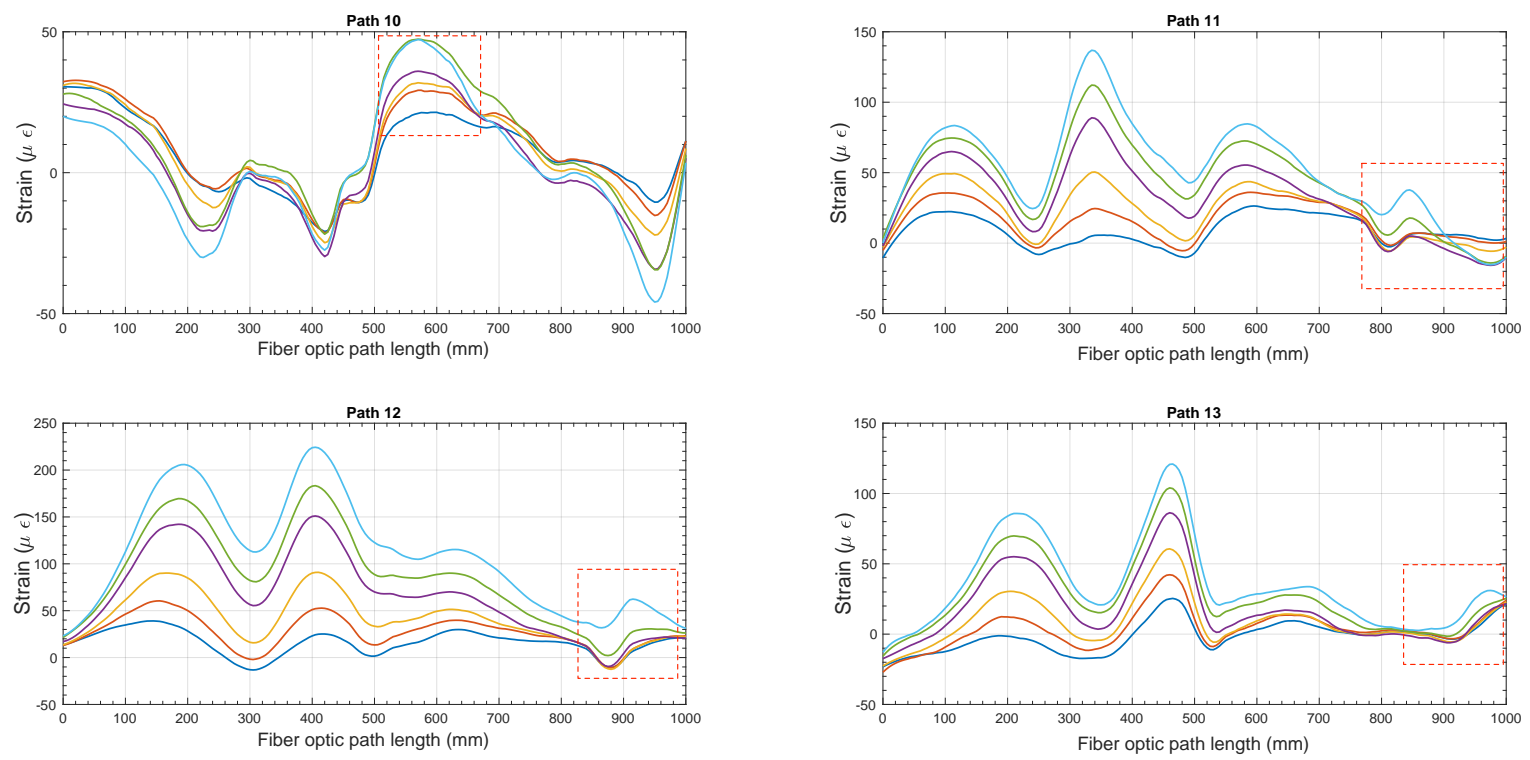

$-120 \mathrm{kN}--140 \mathrm{kN}--160 \mathrm{kN}--180 \mathrm{kN}--185 \mathrm{kN}--195 \mathrm{kN}$

Figure 95. Distributed strain measurements from paths 10,11,12, and 13. Non-linear behaviours of each path are highlighted with a red square (Patricia F. Díaz-Maroto et al., 2018).

From the distributed strain measurements presented above, the first-order derivative was estimated through a finite difference approximation after applying a Savitzy-Golay filter. The non-linear response can be detected at each sensing point by analysing its second derivative regarding every load step. Then the universal threshold was calculated in order to determine the non-linear sensing points from the second derivative squared.

Figure 96 thoroughly represents the first strain derivative of Path 11 (left) and the square of the second derivative (right). As appreciated in the figure, a non-linear behaviour was detected owing to the $-180 \mathrm{kN}$ load step (red square). However, there are also linear sensing points on the same path, whose response against the load is almost linear (green square). 

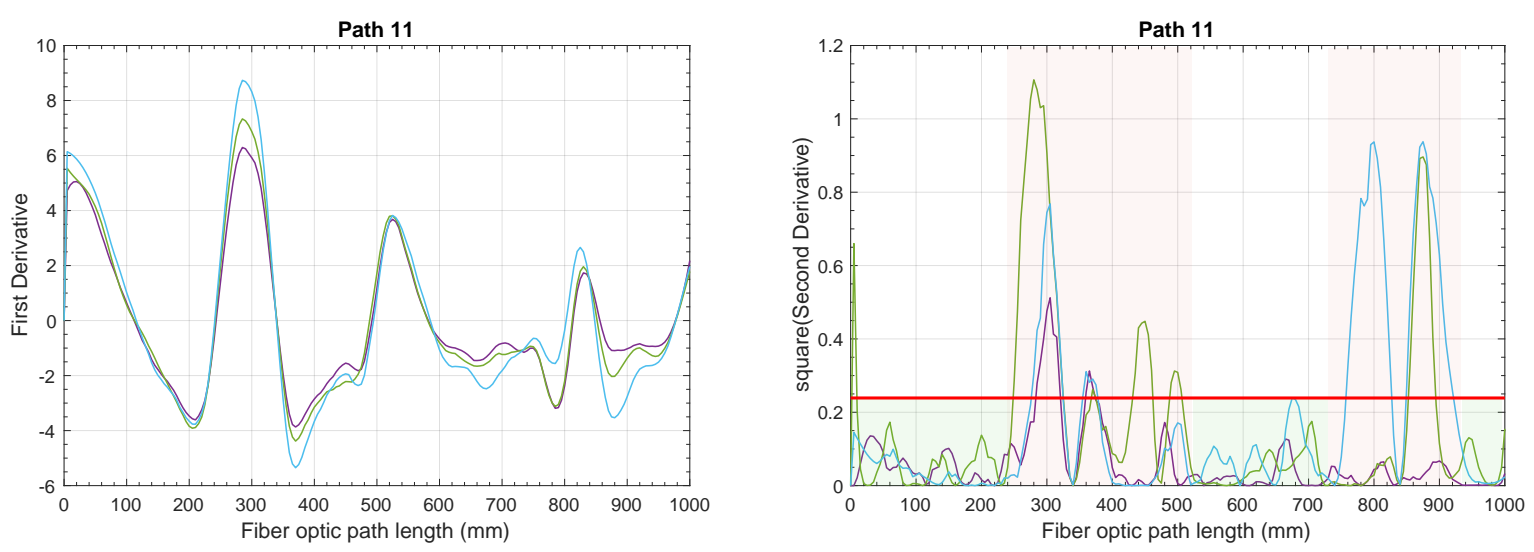

$-180 \mathrm{kN} \longrightarrow-185 \mathrm{kN}--195 \mathrm{kN} \longrightarrow$ Threshold

Figure 96. (Left) First strain field for Path 11. (Right) Second derivative (Patricia F. Díaz-Maroto et al., 2018).

In Figure 97, the full-field strain, first strain derivative, and second derivative measurements are presented on Path 4 (Omega-2 foot). A linear behaviour can be mostly seen over the entire length of the fibre optic path against the load owing to the stiffness of this structural part. Likewise, the shape of the first-order strain derivative remained until the last load step $(-195 \mathrm{kN})$ with some small variations at different points, but still maintaining a low magnitude.
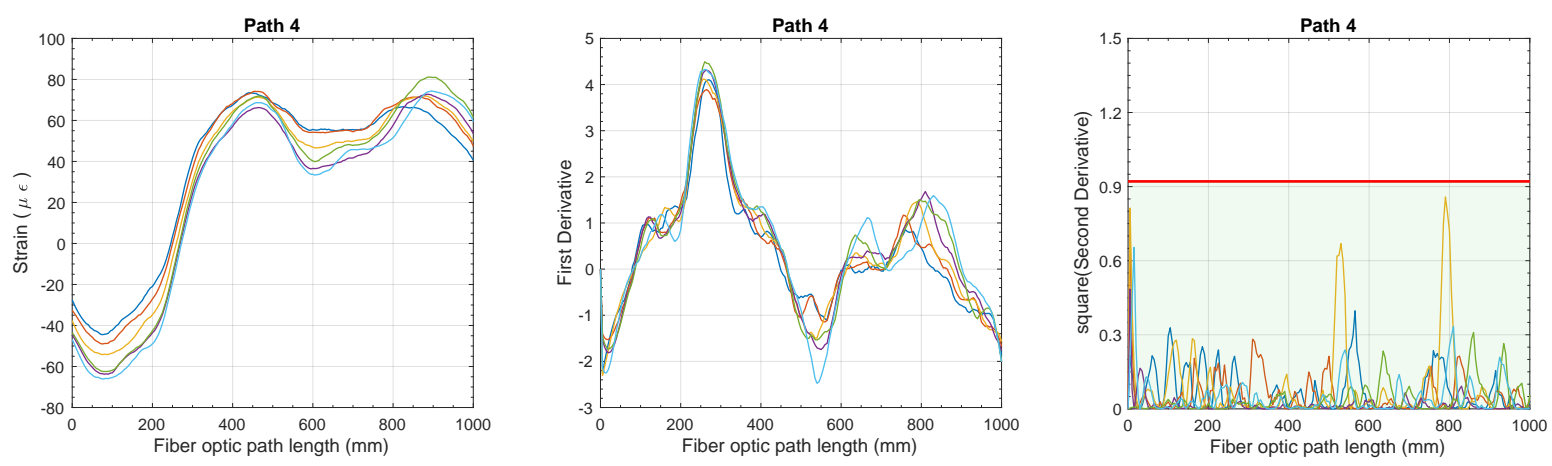

$-120 \mathrm{kN}-140 \mathrm{kN}--160 \mathrm{kN}-180 \mathrm{kN}-185 \mathrm{kN}--195 \mathrm{kN} \longrightarrow$ Threshold

Figure 97. Path 4 distributed strain measurement (left), first derivative (centre), and second derivative (right). (Patricia F. Díaz-Maroto et al., 2018)

Whole buckling analysis conducted in the stiffened panel 1 are presented in Appendix A, Figure 128.

Figure 98 summarizes the results of the buckling analysis based on all distributed strain measurements recorded through the optical network. The contour map of the second derivative at the ultimate compressive load $(-195 \mathrm{kN})$ is represented in Figure 98a, where buckling waves can be clearly recognised because the buckling initiation at a $-185 \mathrm{kN}$ load (Figure 98b) is in agreement with the shear readout of the strain gauge rosette. In addition, 
the sign of the second derivative provides information on the buckling waveform, which can be compared to the images captured by the high-speed camera. What must be stressed is that the areas of the skin panel in which higher values are accumulated on its second derivative were located at bays 4 and 7. Furthermore, at the end of optical fibre path 2, the highest second strain derivative was recorded according to the results of strain gauge rosette R1. Some local points with high strain gradients were located near the frames of the stiffened panel. Figure 98c shows a strain colour map obtained by means of an interpolation of the scattered DFOS data. The highest strain level is obtained in the corners, i.e. bays 3 and 7, of the stiffened panel where the load was being inserted.

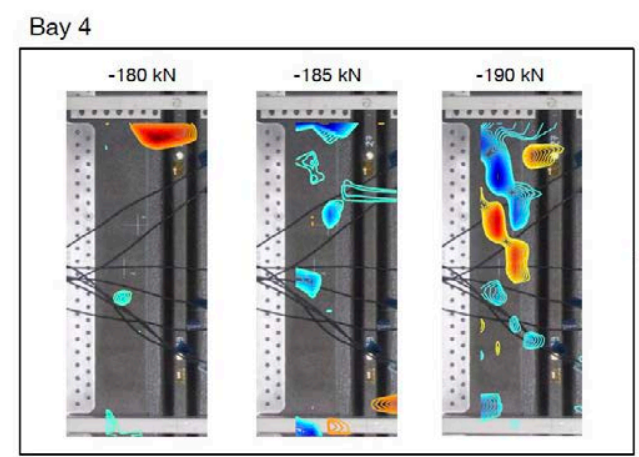

(b)

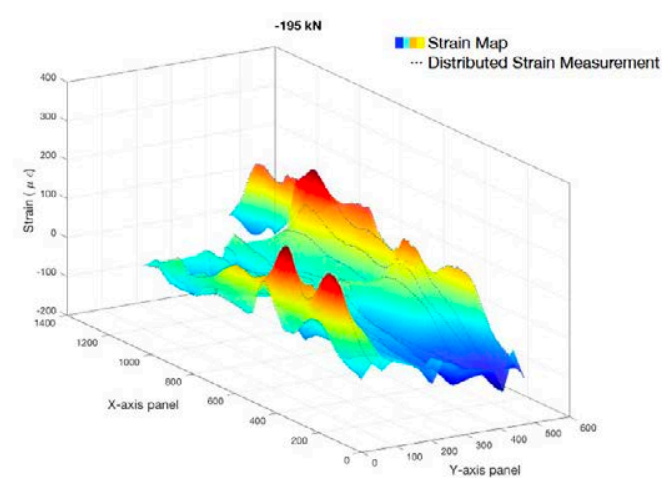

(c)

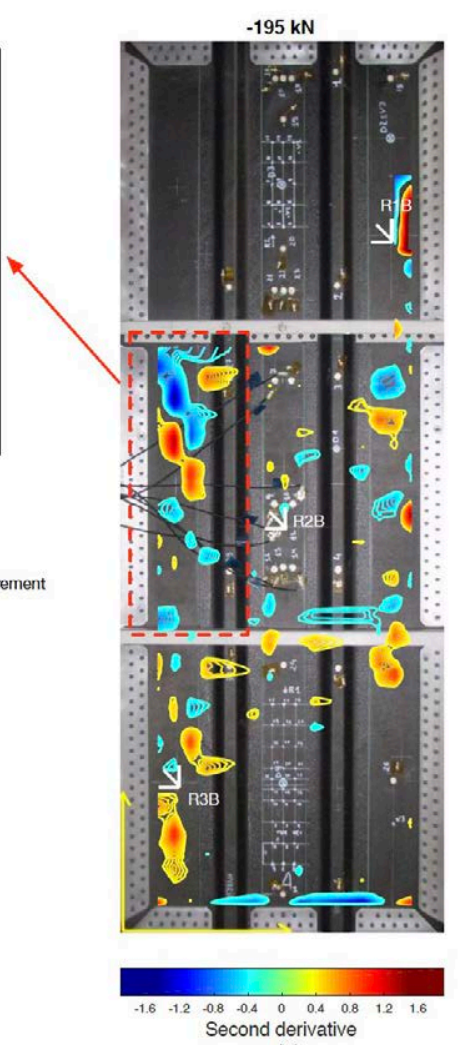

(a)

Figure 98. Summary of distributed strain measurement results (stiffened side). (a) Contour of the second derivative strain map. (b) Detailed buckling appearance of bay 4 from -180 to $-190 \mathrm{kN}$. (c) Strain map at ultimate load (Patricia F. Díaz-Maroto et al., 2018).

\subsubsection{High-Speed Camera}

Throughout the buckling test, the panel was recorded through a high-speed camera. In Figure 99, buckling waves can be seen over the panel skin at a load of $-195 \mathrm{kN}$, which was the last distributed measurement acquired in the buckling panel test, therefore having the same shape as recorded through the distributed measurement in bay 4 . 


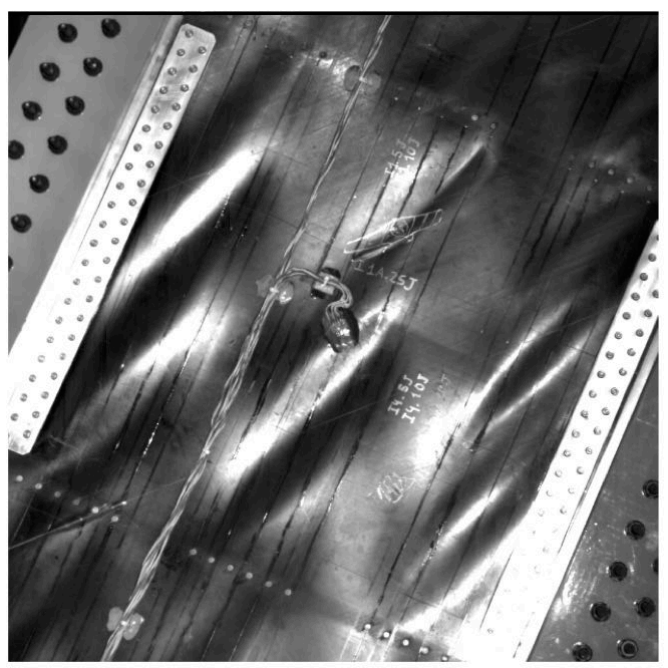

Figure 99. High-speed camera photographs of the stiffened panel at -195 kN (Patricia F. DíazMaroto et al., 2018).

\subsubsection{Conclusions of Panel 1}

A buckling analysis using different sensing techniques was successfully carried out through strain measurements from distributed fibre optic sensors and strain gauge rosettes based on high-speed camera images.

Throughout the structural test, the full-field strain recorded was very complex owing to the experimental setup, this happened because the load direction was not aligned lengthwise to the stiffened panel. This resulted in a set of difficult strain fields to detect the onset of panel buckling. The three pairs of rosettes installed in both a flat and stiffened panel could not monitor more than three locations placed in the main diagonal of the panel. Accordingly, at a compressive load of $-195 \mathrm{kN}$, bay 4 buckled, and the rosettes were unable to detect this behaviour because it was not possible to cover the entire surface of the panel with strain gauges owing to the cost and installation complexity required. The single distributed fibre optic line could detect many potential failure points as a result of the buckling initiation spread over the entire structure. The second strain derivative methodology used to identify these non-linear events opens new post-processing possibilities for the DFOS to measure and evaluate the changes in strain at each sensing point of the fibre optic line. In the same manner, the filter used for the differentiation of the distributed strain measurements allowed a decrease in the local noise of the signal without deteriorating its accuracy. Although the same results were obtained through a high-speed camera, the main obstacle for this technique is its installation when considering the calibration and positioning requirements.

\subsection{Analysis of Stiffened Panel 2}

As presented in the sequence test, this second panel also analysed the growth of the damages after two fatigues lives. For this purpose, the panel was impacted with different energies to carry out different delamination damages over the whole surface of the panel. In contrast to 
the first stiffened panel, this one was tested after different programmed cycles under tensile and compressive fatigue loads. Hence, the full-field strain was compared so as to determine whether the damage had grown, or if it had carried on without increasing. Finally, the buckling test was also performed on this panel for both compressive and tensile load.

\subsubsection{Impact Test}

In contrast to the stiffened panel 1, this panel was damaged by multi-impacts with a barrel in different areas over the panel. The ballistic impact facilities consist of one stage light gas gun, which uses pressurized air to impel the projectile through 8 meters long, $60 \mathrm{~mm}$ calibre barrel, see Figure 100. The multi-impacts consisted on a set of 7-8 balls shot simultaneously.

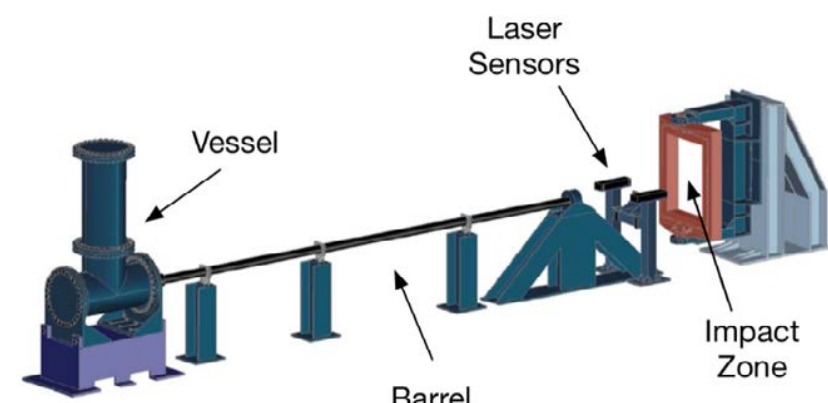

Figure 100. Impact Barrel (Courtesy of University Carlos III of Madrid).

As a consequence, five main damaged areas were defined over the panel which can be appreciated in Figure 101. The most significant area is denoted by damage 1 which is mostly located in the centre bay of the panel. A summary of the damaged area is shown in Table 14.

Table 14. Specification of impacts for stiffened panel 2

\begin{tabular}{c|c|c|c|c}
\hline Impact & Zone & $\begin{array}{c}\text { Impact } \\
\text { Side }\end{array}$ & $\begin{array}{c}\text { Impact } \\
\text { Energies [J] }\end{array}$ & Impactor \\
\hline MUI1 & Bay 5 & Flat & 100 & Barrel (Disperse) \\
\hline MUI2 & Stringer S2 & Flat & 100 & Barrel (Concentrated) \\
\hline MUI3 & Bay 2 & Flat & 100 & Barrel (Concentrated) \\
\hline MUI4 & Stringer S1 & Flat & 100 & Barrel (Concentrated) \\
\hline MUI5 & $\begin{array}{c}\text { Stringer S1, } \\
\text { Bay 3 }\end{array}$ & Flat & 100 & Barrel (Concentrated) \\
\hline
\end{tabular}

The barrel test could be concentrated whose coverage area is of $0.05 \mathrm{~m} \times 0.005 \mathrm{~m}$ or disperse whose coverage area is about $0.5 \mathrm{~m} \times 0.5 \mathrm{~m}$. 

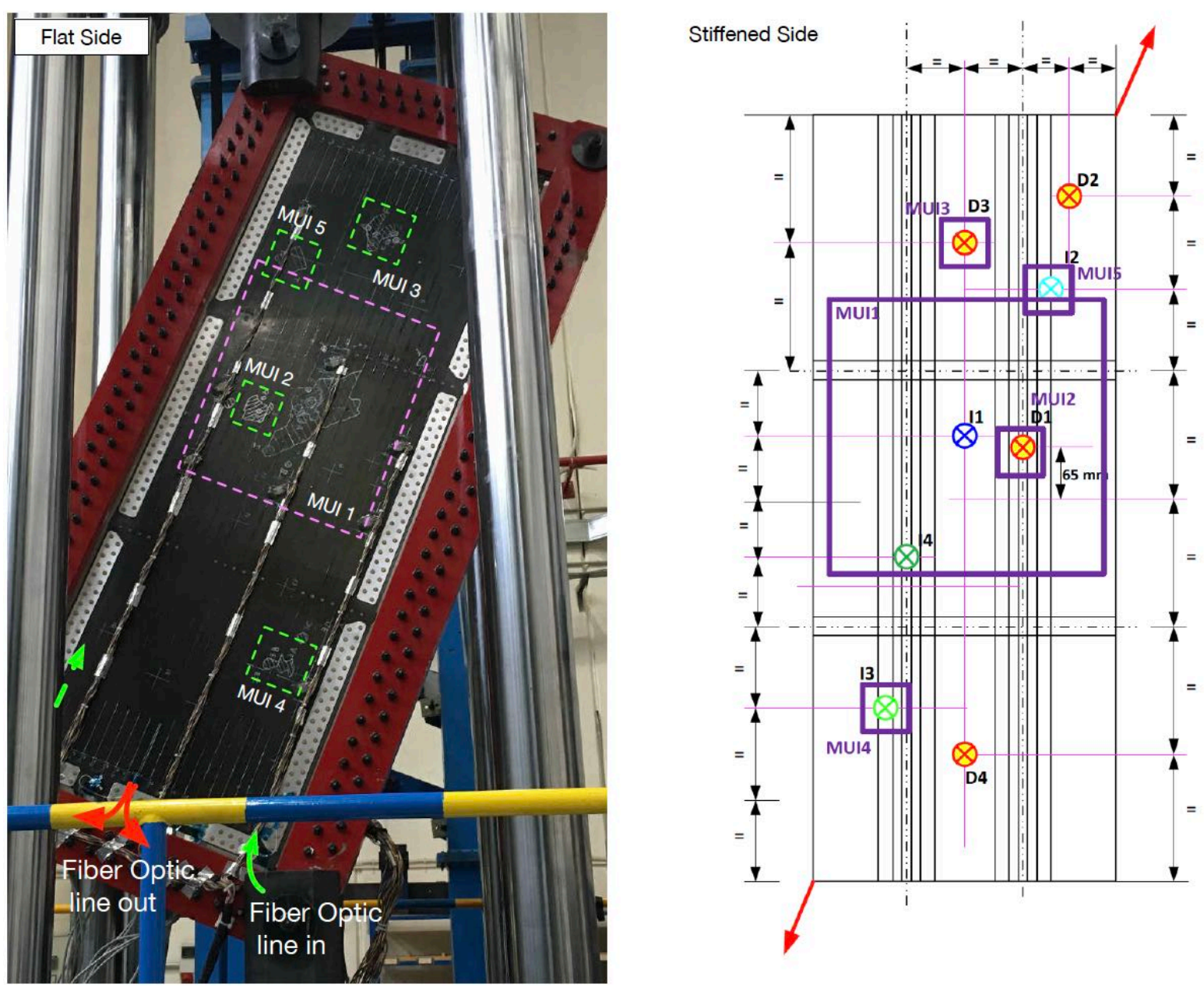

Figure 101. Damages and impact location over the stiffened panel 2.

\subsubsection{Fatigue Test and Damage Growth}

In this section, the fatigue test over the second stiffened panel is presented. The fatigue test was monitored and analysed by means of non-destructive inspections, back-to-back strain gauge rosettes and distributed strain measurements. In the following sections, the comparison of the damage growth through the different technique is presented and described.

\subsubsection{Non-Destructive Testing for Fatigue Test}

As formerly mentioned, non-Destructive tests were regularly carried out at different states of the fatigue test in order to detect a possible increase in the damaged areas. The summary of the results of these inspections are shown in Table 15. 
Table 15. Non-Destructive Testing summary for damage growth during fatigue test.

\begin{tabular}{|c|c|c|c|c|}
\hline \multirow[b]{2}{*}{ Location } & \multicolumn{4}{|c|}{ Damage Characterization } \\
\hline & $\begin{array}{c}\text { Damage Size } \\
{[\mathrm{mm} \times \mathrm{mm}]}\end{array}$ & $\begin{array}{l}\text { Cumulated } \\
\text { cycles }\end{array}$ & $\begin{array}{l}\text { Thickness } \\
{[\mathrm{mm})}\end{array}$ & $\begin{array}{c}\text { Delamination } \\
\text { Depth }(\mathrm{mm})\end{array}$ \\
\hline \multirow{2}{*}{ MUI1 } & 1A: $20 \times 18$ & 38243 & 2.4 (skin) & 0.5 \\
\hline & 1B: $54 \times 13$ & 90000 & $2.4 \pm 1.2$ (skin + stringer $)$ & 2 \\
\hline \multirow{3}{*}{ MUI2 } & $2 \mathrm{~A}: 6 \times 7$ & 38243 & 2.4 (skin) & 1.8 \\
\hline & $2 B: 6 \times 7$ & 38243 & 2.4 (skin) & 1.9 \\
\hline & $2 \mathrm{C}: 20 \times 10$ & 38243 & 2.4 (skin) & 1.3 \\
\hline \multirow{4}{*}{ MUI3 } & $3 \mathrm{~A}: 10 \times 5$ & 38243 & 2.4 (skin) & 1.8 \\
\hline & $3 B: 10 \times 5$ & 38243 & 2.4 (skin) & 1.3 \\
\hline & $3 C: 13 \times 8$ & 38243 & 2.4 (skin) & 1.9 \\
\hline & 3D: $15 \times 10$ & 38243 & 2.4 (skin) & 1.3 \\
\hline MUI4 & \multicolumn{4}{|c|}{ No damage growth } \\
\hline MUI5 & \multicolumn{4}{|c|}{ No damage growth } \\
\hline
\end{tabular}

More detailed damaged areas are shown in the following sections which are also compared with the measurements of the distributed fibre optic paths and rosettes readings.

\subsubsection{Distributed Strain Measurement for Fatigue Test}

As presented in the set-up of the panel, in this case, two fibre optic lines were used to acquire a whole distributed strain measurement over the surface panel. The results here presented included the data after joining both measurements. Furthermore, the areas where the fibre line was not able to be glued to the skin due to the presence of strain gauges or damages were removed from the distributed measurement.

All strain measurements recorded by the OBR at 0 , 45000, 90000, 135000 and 180000 cycles are shown in Appendix ¡Error! No se encuentra el origen de la referencia., ¡Error! No se encuentra el origen de la referencia., ¡Error! No se encuentra el origen de la referencia., ¡Error! No se encuentra el origen de la referencia. and ¡Error! No se encuentra el origen de la referencia., respectively. As expected, the highest strain values correspond to the damaged areas.

First of all, the distributed strain measurement of both path 10 and 11 were analysed in order to ensure the reliability of these measurements despite the fact they belong to different optical fibres. As shown in Figure 82, both paths cover the same area and are parallel with a separation between them in the order of millimetres. A good correlation of strain measurements has been obtained as seen in Figure 102. The coefficient of correlation is in between 0.89 and 0.93 for the different load cases and only a slight difference is appreciated 
at 1 metre of the fibre optic path length. This behaviour is due to the damaged area MUI1 which covers this side (see Figure 82) and hence, high strain concentrations are also accumulated in this area.
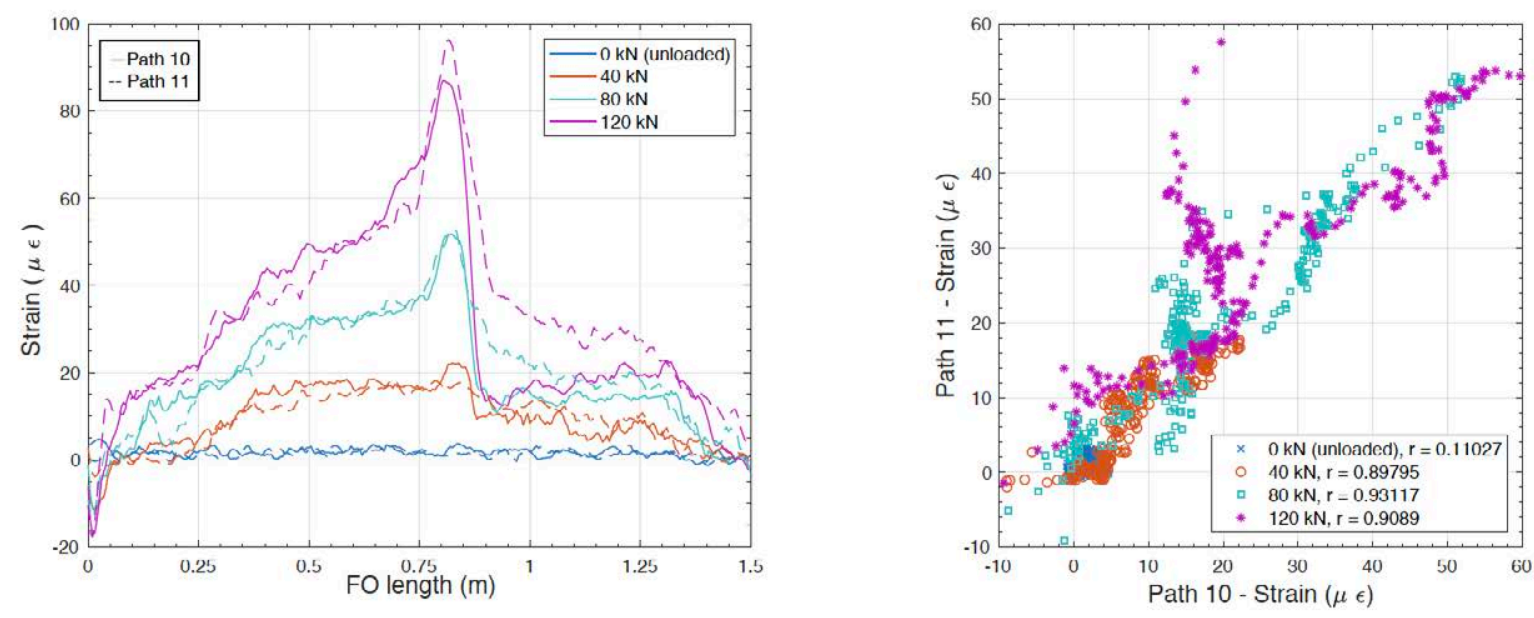

Figure 102. Correlation between path 10 and 11 for tensile test at 45000 cycles.

More scattering is obtained in the compressive test since the correlation coefficient is in between 0.78 and 0.91 (see Figure 103). In this case, the maximum strain value recorded is twice the value than that of the previous tensile test. Despite a good correlation being obtained and the shape of the strain remaining similar in both cases, as can be observed the strain field of the stiffened panel can rapidly change in short distances.
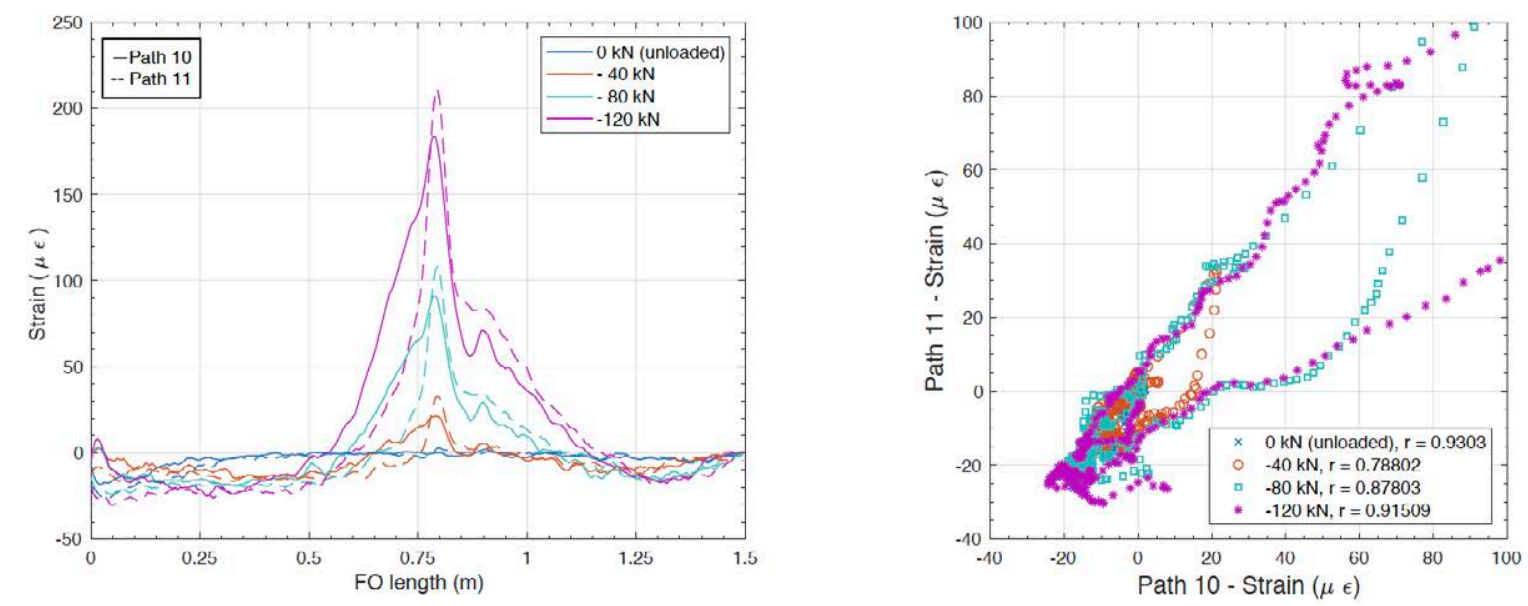

Figure 103. Correlation between path 10 and 11 for compressive test at 45000 cycles.

All distributed measurements were reconstructed to obtain the whole strain map of the stiffened panel for both compressive and tensile load (see Figure 104). In this figure, the distribution of the strain field in the presence of the different damages can be regarded. The most noticeable damage is located at bay 5 and, therefore, the highest strains were recorded around this damage. Furthermore, it must be stressed that under compressive load, the 
damaged area becomes smaller but with high strain concentrations. However, under tensile load the higher strains were aligned with the line of loading. Also, the strain concentration remains at the centre of the panel in bay 5 at MUI1 damaged area.
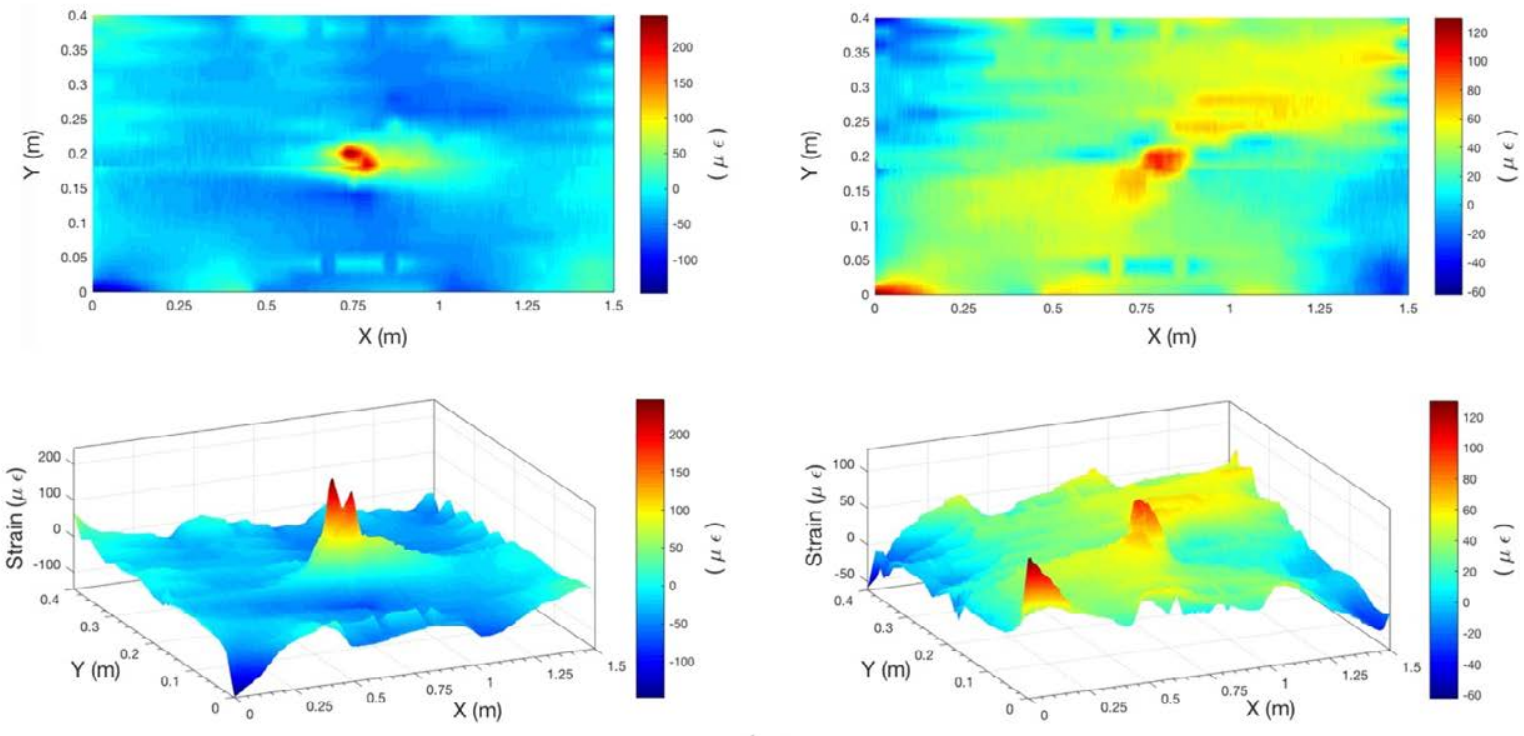

(a)

(b)

Figure 104. Strain colour map under $-120 \mathrm{kN}$ of compressive load (a) and $120 \mathrm{kN}$ of tensile load (b) after 45000 cycles.

The growth of the different damages defined in 6.6.1, is analysed through the full-field strain recorded after the different fatigue cycles at load steps $\pm 40 \mathrm{kN}, \pm 80 \mathrm{kN}$ and $\pm 120 \mathrm{kN}$.

The general results obtained for the compressive test at different fatigue cycles are shown in Figure 105. These measurements are related to the measurement at 0 cycles which is considered as a reference measurement (including damages). Then, the results show the difference between the distributed strain measurement obtained at such cycle and the distributed strain measurement recorded at 0 cycles. It must be emphasized that the higher residual strain level is obtained at the end of each FO line, i.e. at the egress of the optical fibre (paths 10 and 11), which corresponds to the centre bays and the MUI1 damage location. What can be observed in this figure, in general, is the strain difference for FO line 1 oscillates between $145 \pm 10 \mu \varepsilon, \pm 15 \mu \varepsilon$ and $\pm 20 \mu \varepsilon$ for $-40 \mathrm{kN},-80 \mathrm{kN}$ and $-120 \mathrm{kN}$ respectively. 

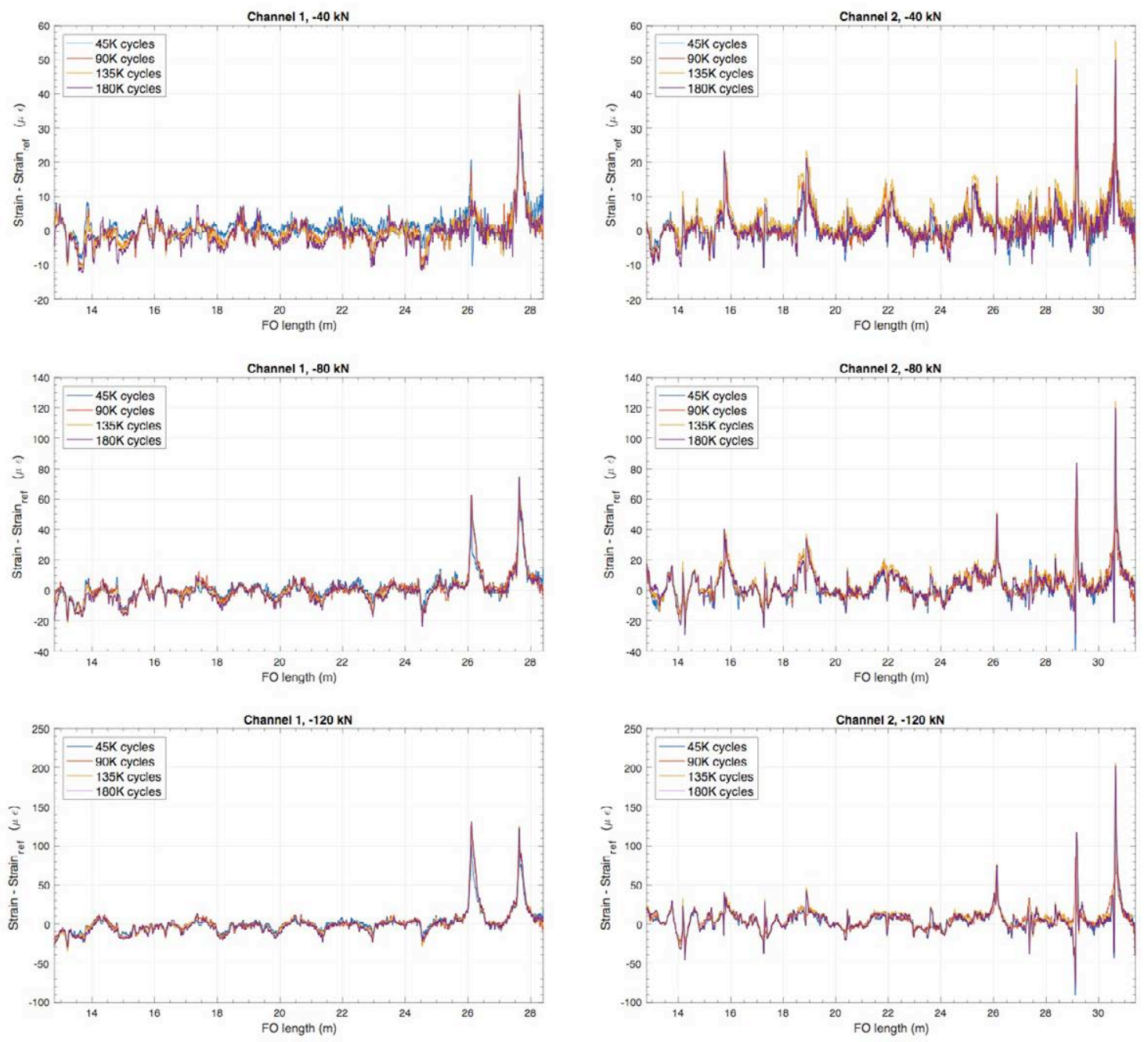

Figure 105. Comparison of the total of distributed measurements recorded at 45000, 90000, 135000 and 180000 cycles under $-40 \mathrm{kN},-80 \mathrm{kN}$ and $-120 \mathrm{kN}$ of compressive loads.

Same measurements can be obtained for the tensile test (see Figure 106). The main aspect that should be highlighted is the lower level of the strains regarding the compressive test. In this case, the damage MUI1 is not appreciated as it had occurred in the compressive test presented above. Therefore, as was to be as expected, in the view of these results, the damages are prone to grow under compressive loads. Additionally, at 90000 cycles, there is an offset of the measurement regarding the rest of the measurement. 

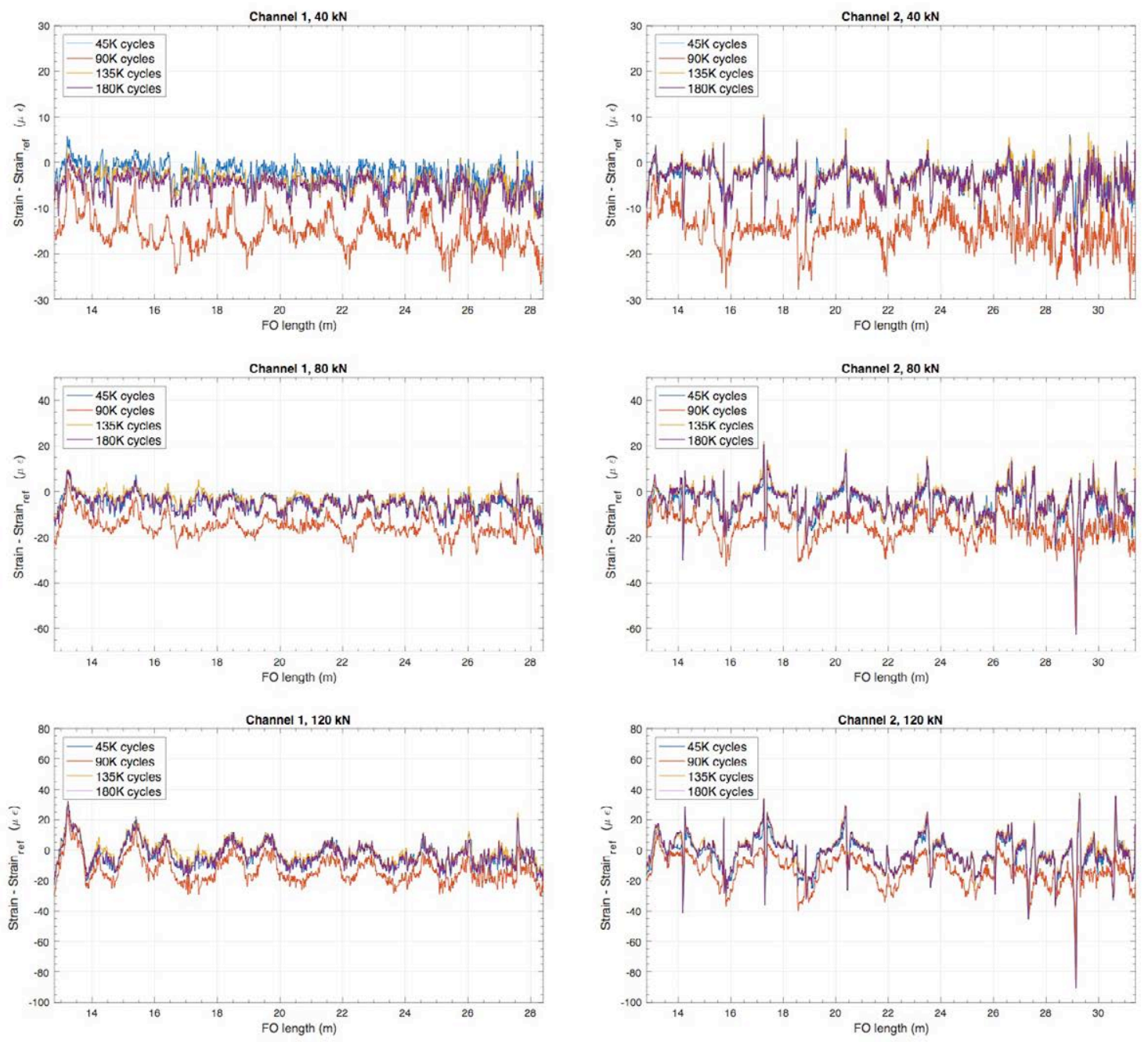

Figure 106. Comparison of the total of distributed measurements recorded at 45000, 90000, 135000 and 180000 cycles under $40 \mathrm{kN}, 80 \mathrm{kN}$ and $120 \mathrm{kN}$ of tensile loads.

The growth of these damages will be thoroughly analysed in the following sections, especially under compressive test measurement since they are prone to grow under compressive loads. Moreover, results from non-destructive tests and rosettes strain readings at different cycles were used to be able to compare to those obtained with distributed fibre optic measurements.

\subsubsection{Analysis of Damage MUI1 and MUI3}

The damage noted by MUI1 and MUI3 can be analysed through path 9 to 13 since these paths cross both damages. The distributed strain measurements recorded in these paths at different cycles are shown in Figure 107. As observed, the damage MUI1 has significant differences after 45000 fatigue cycles at path 9, 10 and 11. Likewise, MUI3 damaged area hardly differed during the different cycles. 
MUI1 Damaged Area

MUI3 Damaged Area
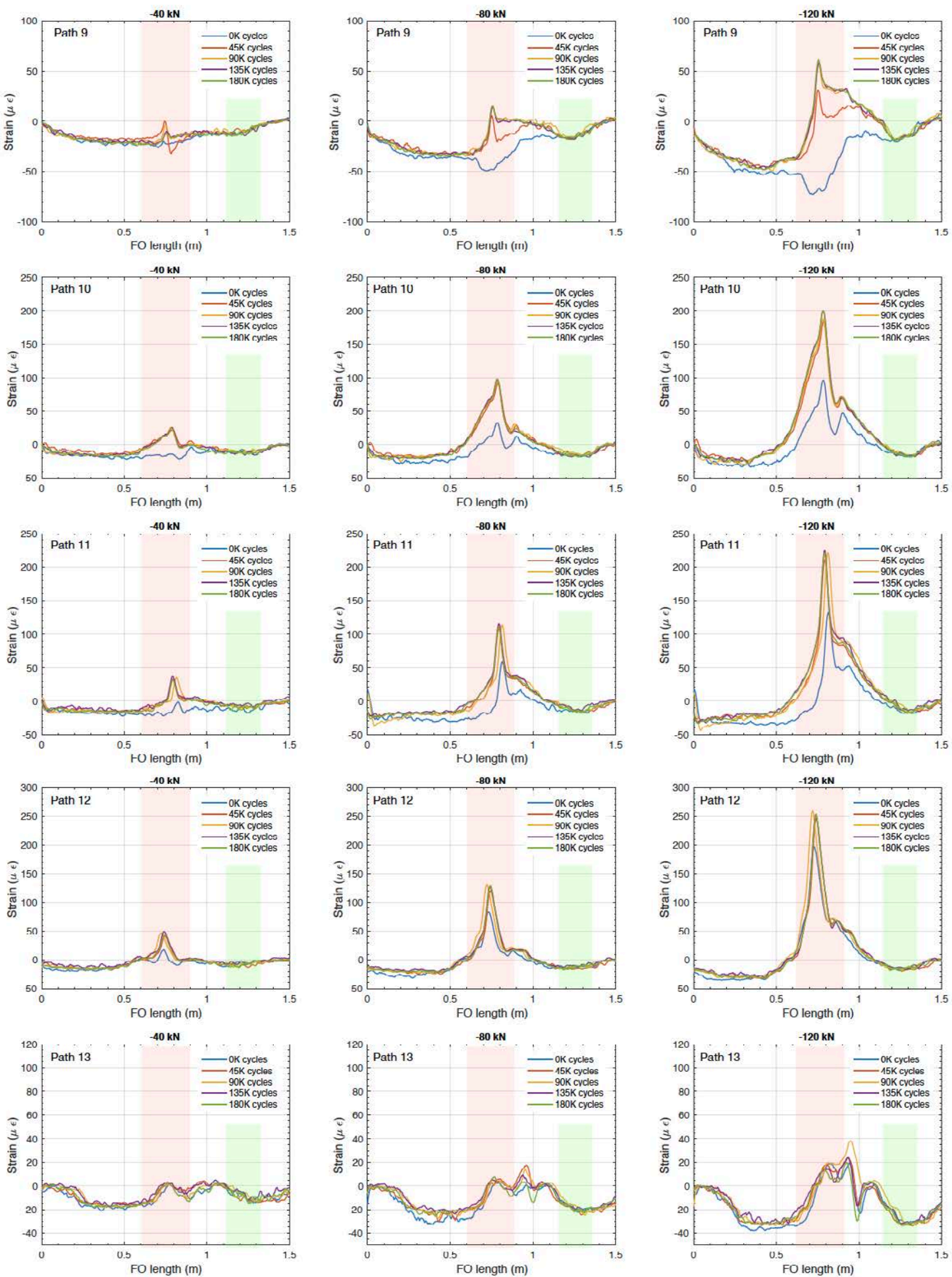

Figure 107. Distributed measurement around damage MUI1 analysed by path 10, 11 and 12 .

The NDT inspection detected an increase of the damage MUI1 at 38243 and 90000 cycles in the areas $1 \mathrm{~A}$ and $1 \mathrm{~B}$, respectively (see Figure 108). The growth of the $1 \mathrm{~B}$ damaged area can be 
appreciated in the distributed strain measurement for path 9 since it is located very close to it as shown in Figure 107. Thus, after both 45000 and 90000 cycles, the strain level at path 9 experienced a significant increment around MUI1 damaged area (highlighted with a red square) which remained for 135000 and 180000 cycles, which can be observed below.
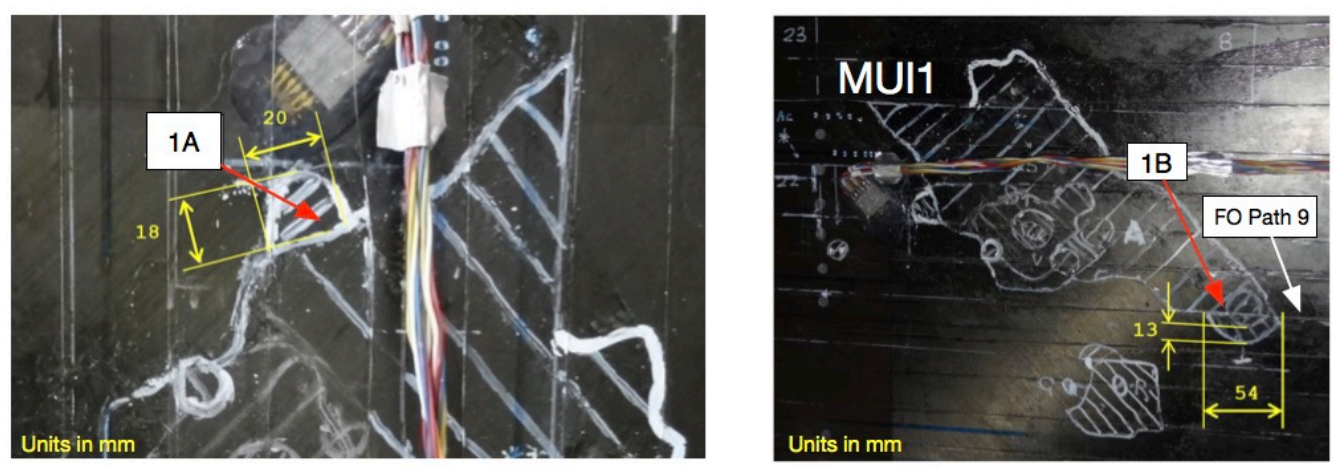

Figure 108. Growth of damage MUI1 at 90000 cycles.

This damage growth was also noticed by the rosette 4 , which was located very close to this damage as can be seen in Figure 108, and also by the rosette 5. Regarding the side R4A-A, i.e. $0^{\circ}$ and flat side, the difference between the 0 cycles and $45 \mathrm{~K}$ cycles has a good agreement with the strain increment on path 12 under $-120 \mathrm{kN}$ (where this rosette is located).

The same behaviour is obtained for rosette 5, but strain readings are not as high as those for rosette 4. Furthermore, an increase of strain in R5B-A under $\pm 120 \mathrm{kN}$, i.e. $0^{\circ}$ and stiffened side, after $180 \mathrm{~K}$ cycles must be noted.
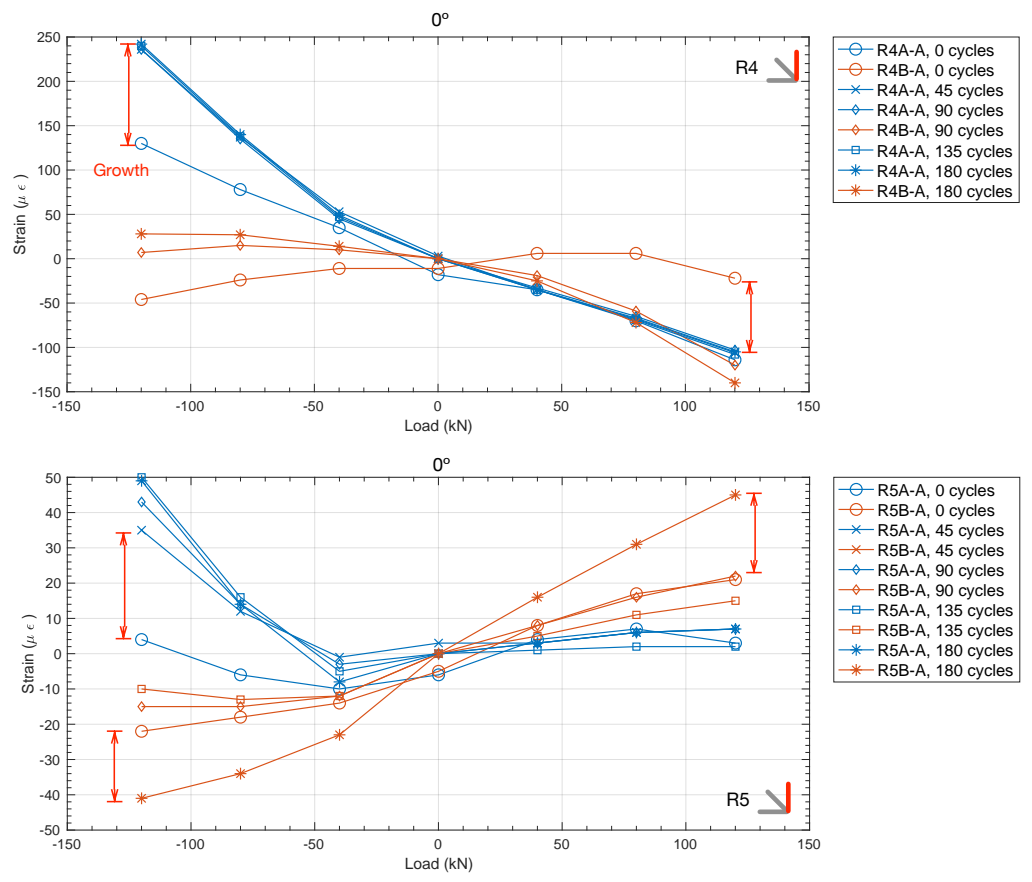

Figure 109. Strain reading at $0,45 \mathrm{~K}, 90 \mathrm{~K}, 135 \mathrm{~K}$ and $180 \mathrm{~K}$ cycles for rosette 4 and 5. 
Regarding the MUI3, NDT inspection detected the small growth of damage. However, they were too small (hardly $6 \times 7 \mathrm{~mm}$ ) to release significant residual strain to be captured by the optical fibre since they are in the order of the gauge length and sensor spacing parameters. No rosette was located close to this damage to analyse the residual strain.

\subsubsection{Analysis of Damage MUI2}

The growth of the damage MUI2 had an important increase in the full-field strain on path 8 at 45000 cycles, see Figure 110. Furthermore, a slight difference in the distributed strain measurement can be seen on path 6 and 7. It must be stressed that this damage is located at the foot of the stringer and it corresponds with a very stiffened area as can be seen in the overall level of the strain during the different load tests. Despite this fact, the growth of the damage after $45 \mathrm{~K}$ cycles was successfully detected by the distributed strain measurement as presented here below.
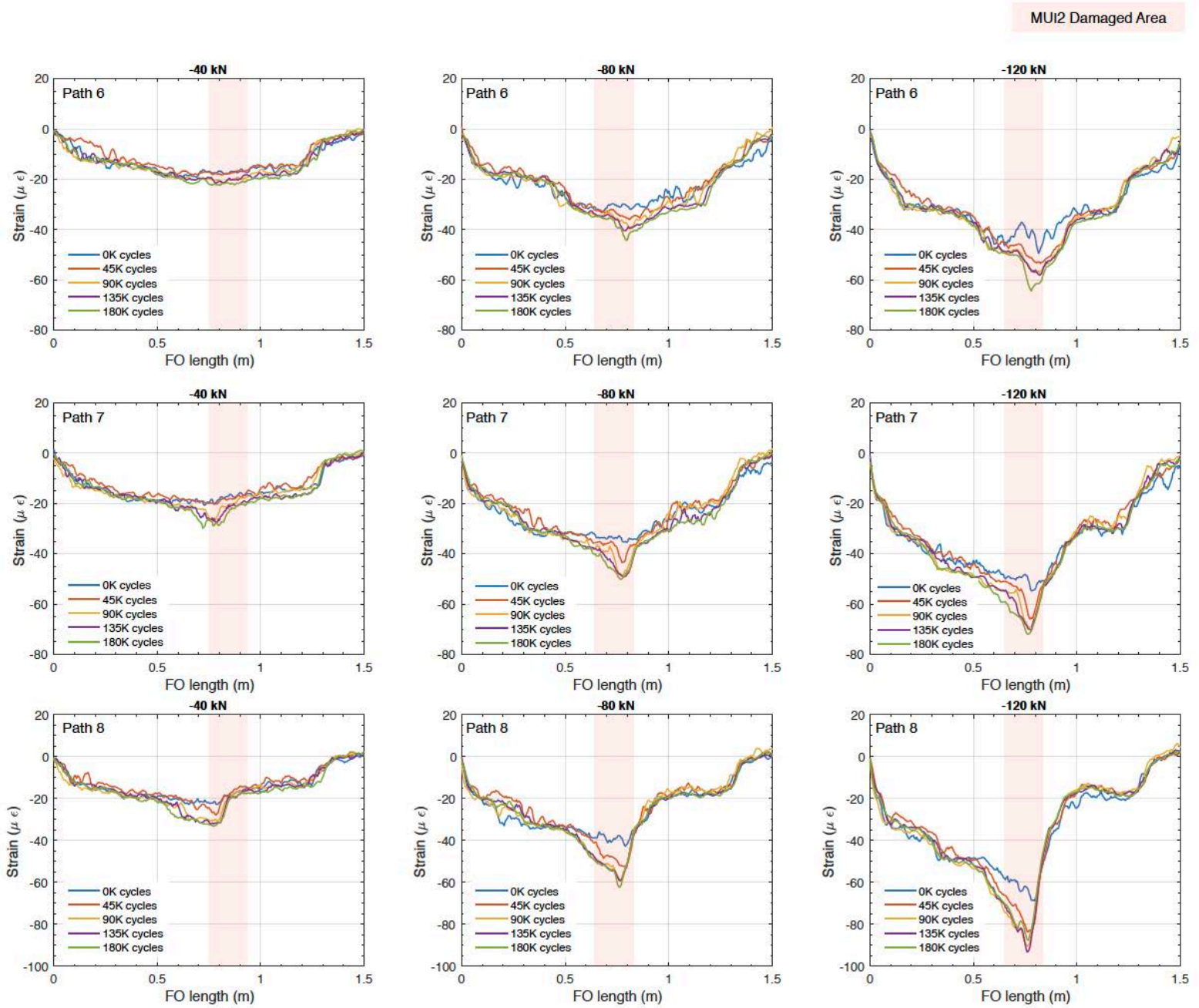

Figure 110. Distributed measurement around damage MUI2 analysed by path 6, 7 and 8. 
Regarding the NDT measurement, the growth of the different damages is shown in Figure 111 , where three different delaminated areas were denoted by $2 \mathrm{~A}, 2 \mathrm{~B}$ and $2 \mathrm{C}$. Firstly, the delamination $2 \mathrm{~A}$ is very slightly appreciated on path 7 since the growth of this damage is hardly $6 \times 7 \mathrm{~mm}$. As occurred in MUI3, the gauge length of the distributed measurement was $5 \mathrm{~mm}$ for these measurements which made the detection of the damages in this order quite difficult. Therefore, in order to locate this increment in the strain field, the search should be limited to one single sensing point. Furthermore, path 6 was not located close to any damage growth detected by the NDT. However, it is able to detect small changes in the distributed measurement but in the case of path 7 or 8 it is not as obvious. Finally, represented in this figure, damage growth $2 \mathrm{C}$ is the most significant around MUI2 area with $20 \times 10 \mathrm{~mm}$. It was also detected by the distributed strain measurement on path 8 , which recorded a difference between 0 cycles and $45 \mathrm{~K}$ cycles of $\pm 20 \mu \varepsilon$.

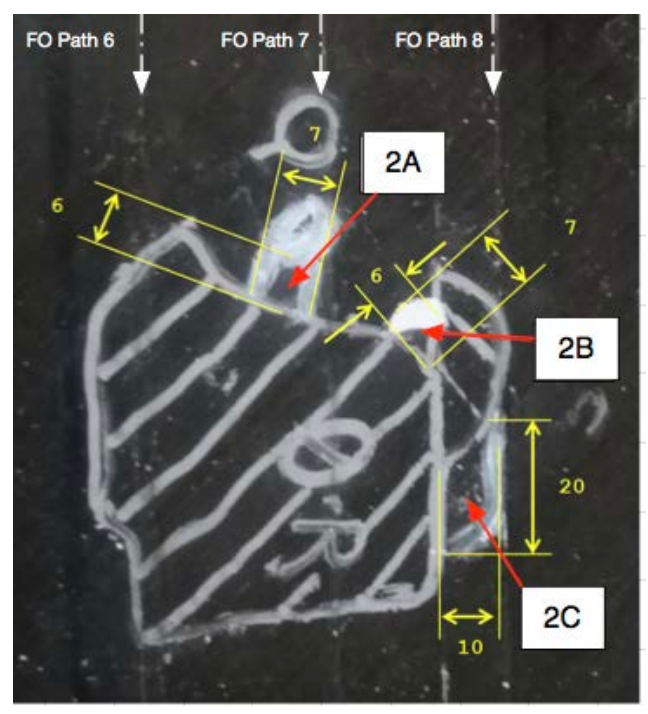

Figure 111. Growth of damage MUI2 at 38243 cycles.

Therefore, a good agreement between NDT and distributed strain measurement was obtained by analysing the distributed strain measurements even if they had low strain level.

\subsubsection{Analysis of Damage MUI4}

In the same way that occurred for MUI2, the strain field recorded around MUI4 is much smaller due to this damage being located on the foot of the omega stringer, observed in Figure 112. Despite this fact, around the damaged area a slight increase of the strain level was recorded after 45000 cycles.

Regarding the NDT results, no discernible damage was appreciated at MUI4. 

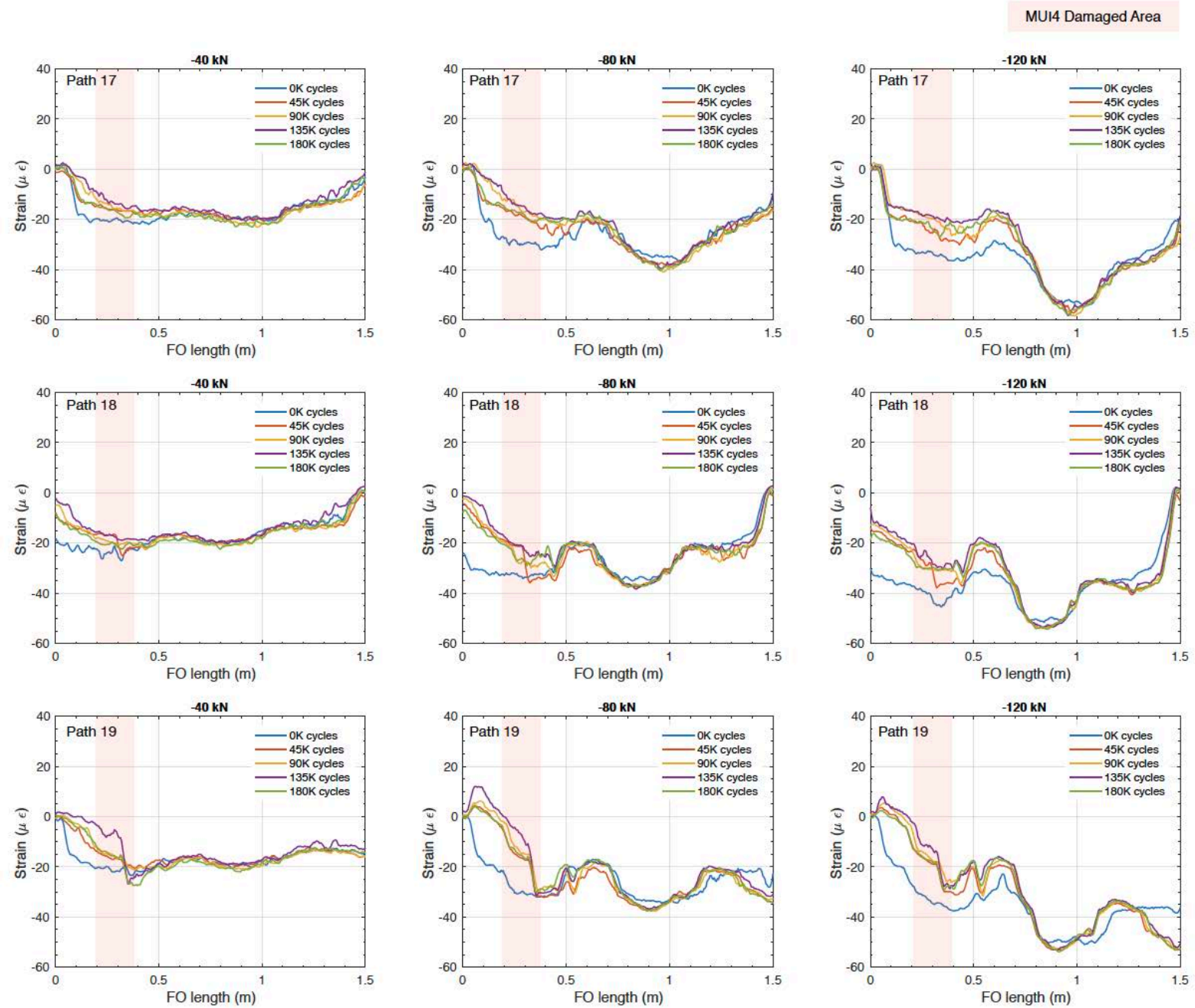

Figure 112. Distributed measurement around damage MUI4 analysed by path 17, 18 and 19.

\subsubsection{Analysis of Damage MUI5}

As was predicted for the NDT analysis the MUI5 has not grown, see Figure 113. Regarding the measurement recorded by the distributed fibre optic network at this area, the full-field strain remains at the same level and shape in the course of all the different cycles. Therefore, no damage growth can be confirmed considering these results.

Only, slight differences are appreciated at the centre of the measurement that can be caused by the presence of the damaged area MUI2. 

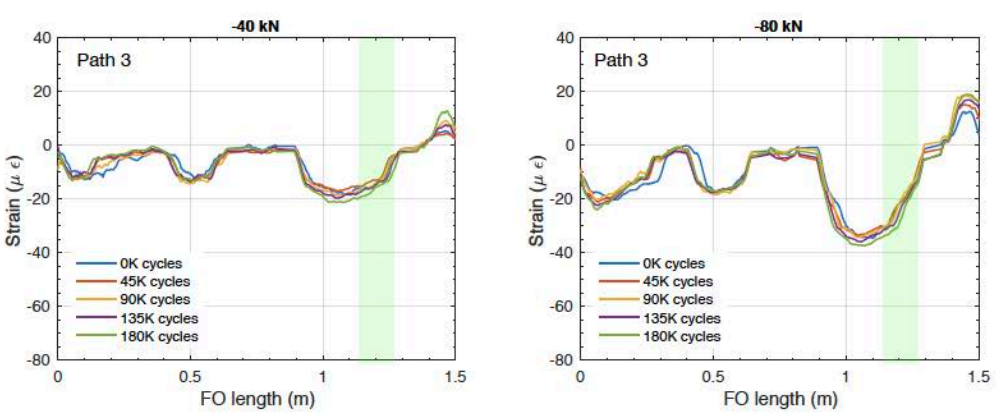

MU15 Damaged Area
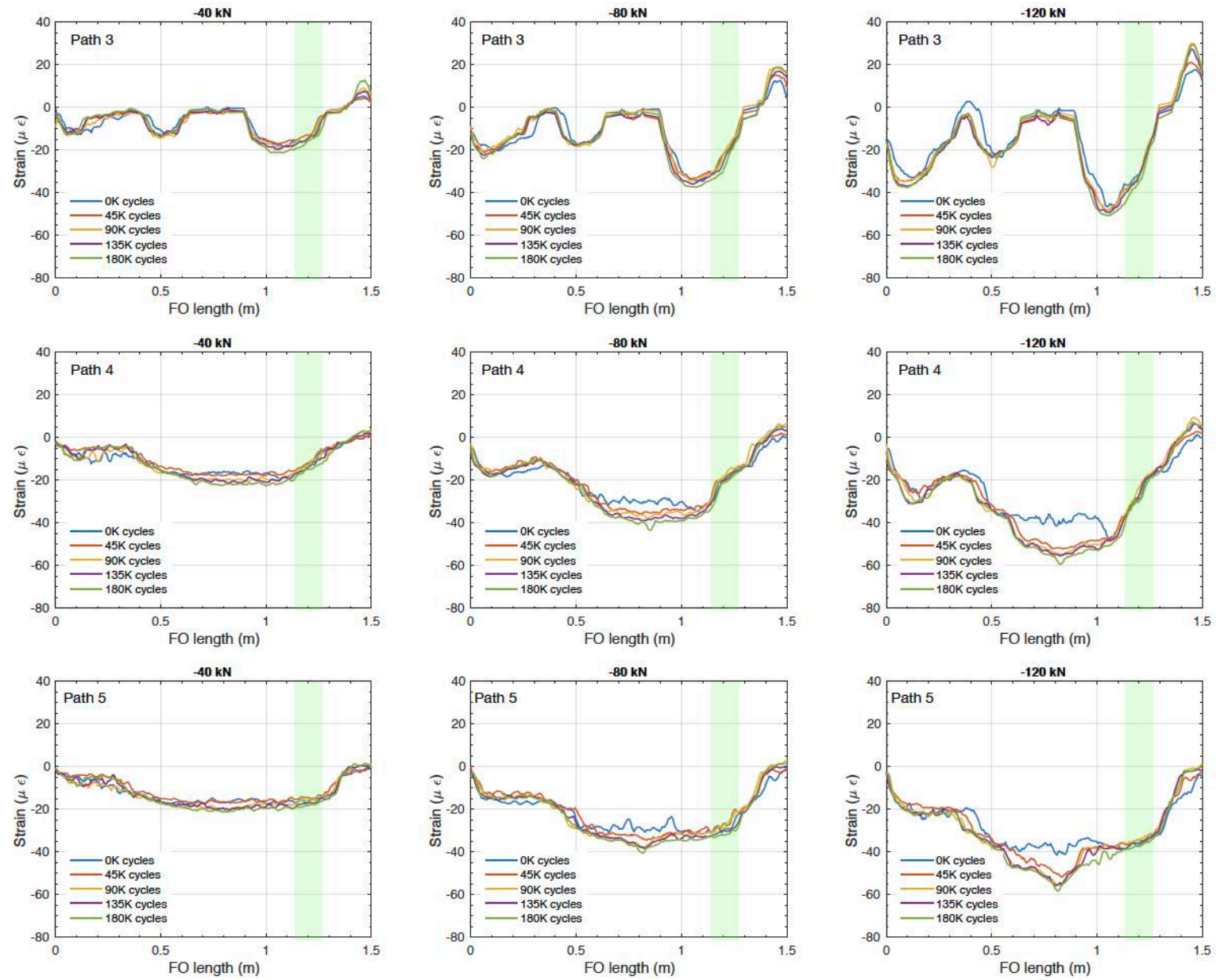

Figure 113. Distributed measurement around damage MUI4 analysed by path 3, 4 and 5 .

\subsubsection{Summary of damage growth}

As presented in the previous section, the most critical damage which presented the most significant residual strain was obtained in MUI1 after 45000 cycles in a large area, which can be observed in Figure 114. This figure shows the residual strain field at each of the considered cycles. Therefore, the reference for each figure is just the previous acquisition. Then, from 45000 cycles to 13500 cycles, local areas around MUI1 damage can be distinguished with very high residual strains in a large area at the centre of the panel. It must also be emphasized that from $135 \mathrm{~K}$ to $180 \mathrm{~K}$ of fatigue cycles, no representative residual strains were obtained at this area which means that no-damage growth was recorded.

Due to the high strain level of the residual strains for MUI1 damage, the rest of the damages analysed are hidden in this visualization. Furthermore, as described above, the residual strain level is too small, and it cannot be compared with the residual strain of $80 \mu \varepsilon$ of this damage. 


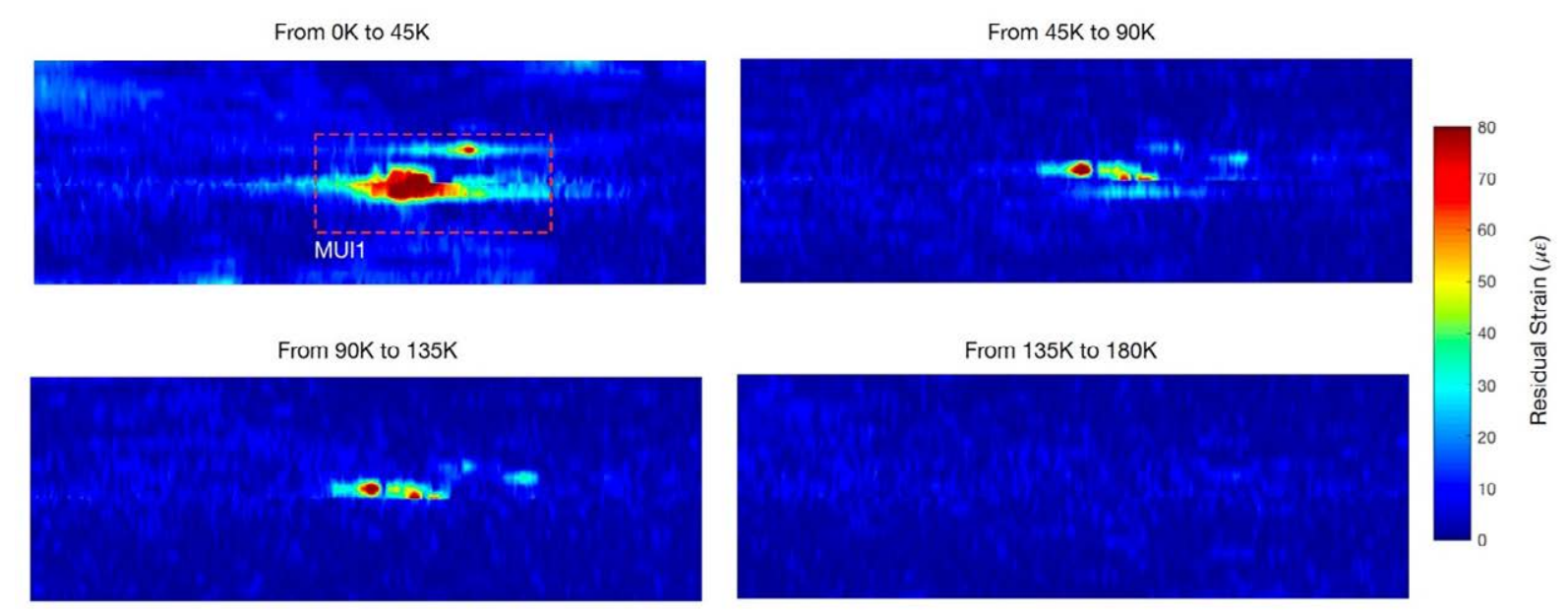

Figure 114. Distributed map of residual strain during fatigue test.

In addition, the distributed strain measurements were able to detect changes even in very stiffened areas, such as stringer foots. The strain level in these areas were too small, but as was proven, delamination releases strain which made it detectable for the optical fibre.

An overall good agreement was obtained regarding the NDT results. Not only in the prediction of the cycle at which the damage grew, but also in the specific location just as occurs with damage MUI2. Regarding the strain readings of the rosettes, only the rosette 4 and 5 could be used to analyse the damage growth since it was located very close to it. This technique is not suitable to measure the residual strains in order to detect significant changes because if the damage occurs in the far field the rosette is not able to detect these changes.

Another fact that must also be commented is that the distributed fibre network was placed after the impact test (on the flat side) and the damage growth was identified by changes in the residual full-field strain. In a real scenario, the structure to be monitored should be health with no-damages. Then, the sensing network must be placed on the side where it is unlikely to incur damages because otherwise, the fibre can be broken, and a reparation will be needed. Taking into account that the installation of the fibre is to ensure its survivability, distributed fibre optic sensors have been proven to be a good choice to monitor the health of a large structure from the distributed strain measurement at different states.

\subsubsection{Buckling Analysis}

After two fatigue lives for the stiffened panel, a buckling analysis was carried out under a compressive load of up to $-235 \mathrm{kN}$ and then, under a tensile load of up to $300 \mathrm{kN}$ was carried out. This test aims to detect not only the buckling appearance but also, to monitor the growth of the damages. 


\subsubsection{Distributed Strain Measurement Results}

Firstly, the distributed strain measurements for both fibre optic channels are shown in Figure 115 and Figure 116. Channel 1 includes from path 1 to 10 and channel 2 includes from path 11 to 22. In the view of this preliminary distributed measurement results, a symmetrical behaviour is obtained as was to be expected. The highest strain values are recorded at the end of the paths, which correspond to the damaged area in the centre of the bay 5 . Additionally, it must be noted that all these paths recorded tensile strains (positive), whereas the other paths recorded compressive strains (negative). This gives information about the deformation of the panel under diagonal compressive and tensile load. The loops and the ingress/egress were removed from this representation in order to show only the effective length of the optical fibre which was analysed. Whole distributed measurements recorded throughout the buckling test at the different paths are collected in Figure 129.

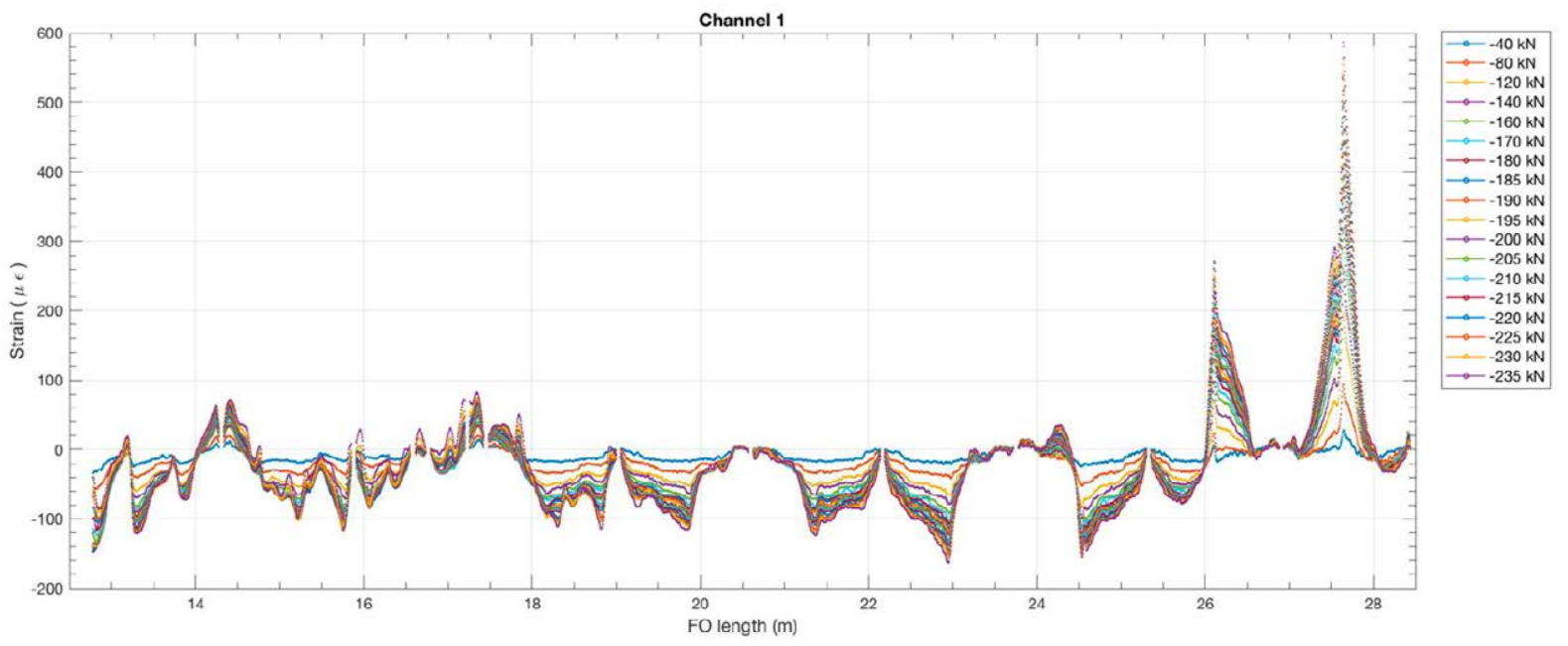

Figure 115. Complete distributed measurement under compressive load for buckling test acquired by the fibre optic channel 1 . 


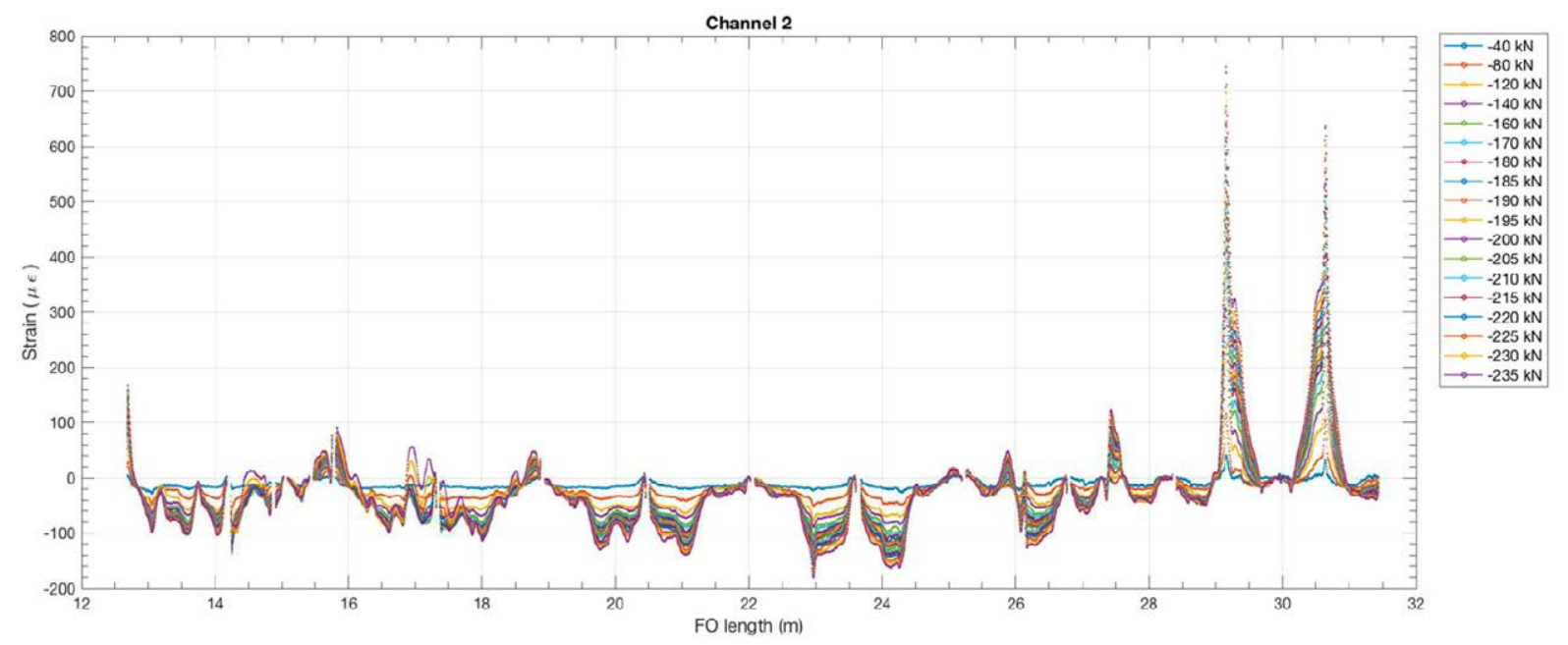

Figure 116. Complete distributed measurement under compressive load for buckling test acquired by the fibre optic channel 2 .

As was obtained during the fatigue life tests, the damage MUI1 at the centre of the panel accumulated the highest strain level $(700 \mu \varepsilon)$, observed in Figure 117. Due to the high strain concentrator recorded in this damage, other behaviours in the panel are hidden in this visualization. Further discussion of the results obtained for this test will be performed in the next paragraphs.
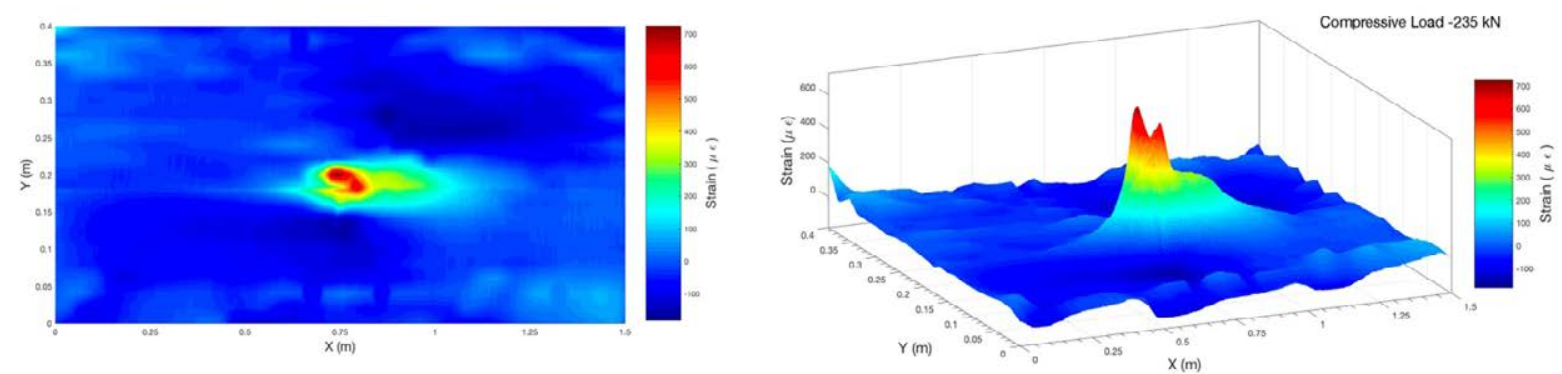

Figure 117. Full-field strain for $-235 \mathrm{kN}$ of compressive load.

\subsubsection{Strain Gauge Rosettes Results}

The comparison of the measurement between the rosettes and the distributed measurement were carried out through the scattered interpolant function. Firstly, a surface of strains was reconstructed by the whole distributed measurement recorded throughout the experimental test. Hence, each strain gauge rosette was positioned regarding the stiffened panel and the simulated strain value at that location was obtained through the interpolation of the strain surface.

During the buckling test only rosettes R2, R4 and R5 were able to obtain a readout in the fibre lengthwise, i.e., the $0^{\circ}$ degree. As observed in the Figure 118, a good agreement was obtained in the correlation of rosettes R2A and R4A with those simulated by the distributed 
measurement (denoted by "sim R2A" and "sim R4A"). However, the simulated rosette R5A, i.e. "sim R5A", has significant deviation from the real readout of the rosette during the experiment.

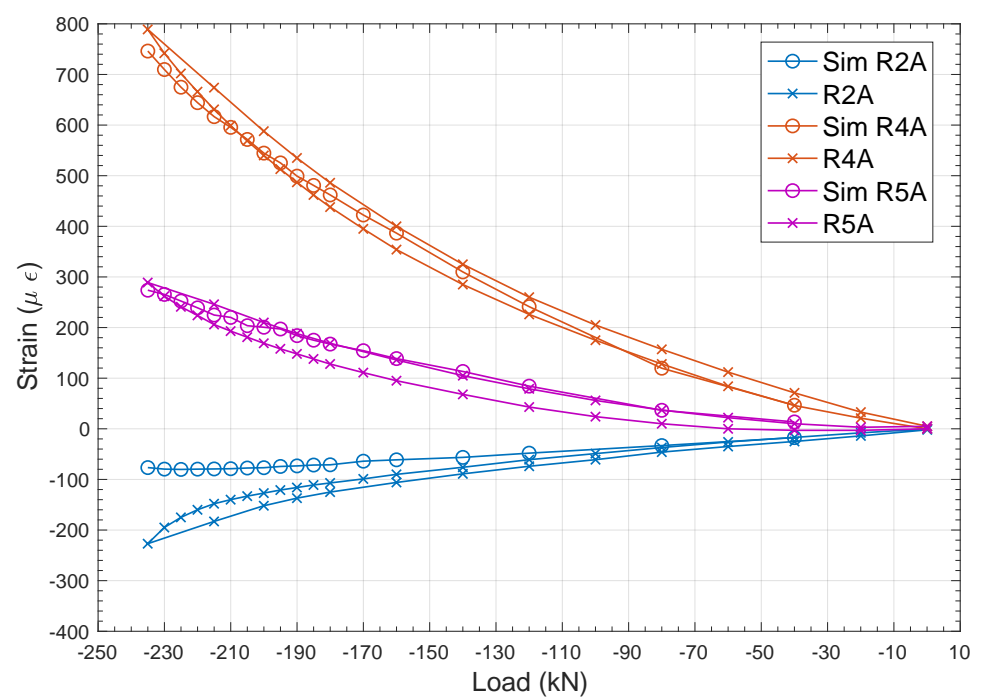

Figure 118. Comparison of compressive buckling test between rosettes and distributed measurement.

During the buckling test under diagonal compressive load, the strain readings of the rosettes at the $0^{\circ}$ direction, i.e. the fibre optic line direction, can be compared as shown in Figure 119. For the read-out of rosette R4, a different behaviour on both sides can be appreciated since the load-strain curves increase rapidly on the flat side. This effect is mostly due to the damage located at the centre of the bay 5 and, more specifically, due to the delaminated area generated during the fatigue test as explained in 6.6.2. Despite this effect, it cannot be confirmed that the structure at this point was buckled from the rosette strain readings. In the same way that occurred with distributed measurement, the recorded strains in the flat side for this path were positive, i.e. tensile strains. However, the R2 and R5 nearly reproduced the same symmetrical behaviour on both sides of the panel. It must stressed that R2 sharply increased and lost the strain-load linearity at $-215 \mathrm{kN}$.
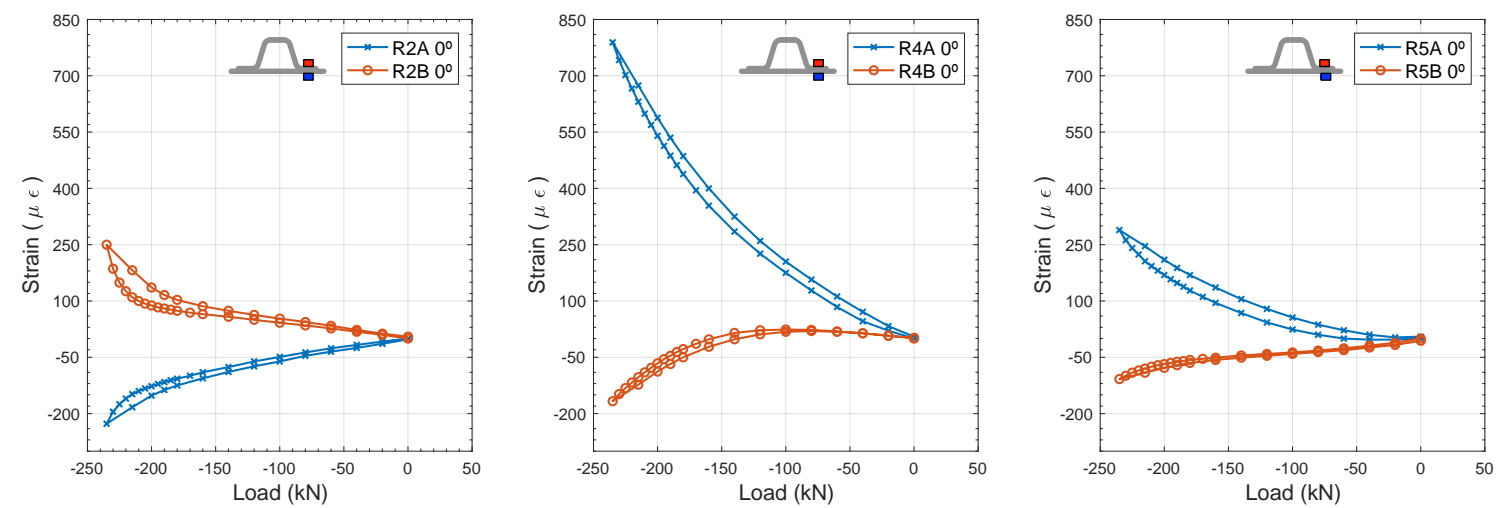
Figure 119. Rosettes strain gauge comparison at $0^{\circ}$ for buckling test under compressive load.

Throughout the tensile load test, the read-out of the rosettes 2, 4 and 5 are displayed in Figure 120. As occurred to the same degree in the compressive load test, the R2 shows a linear behaviour against load, recording roughly the same level of strain on the stiffened side but much a more lower level on the flat side. The same behaviour was also obtained from R4 rosette, where the stiffened side underwent a faster strain increase comparing it to the flat side where the fibre optic was placed. Finally, the R5 shows a different behaviour regarding the compressive test since the two measurements are under tensile strains. This suggests that the influence of damage caused this behaviour.
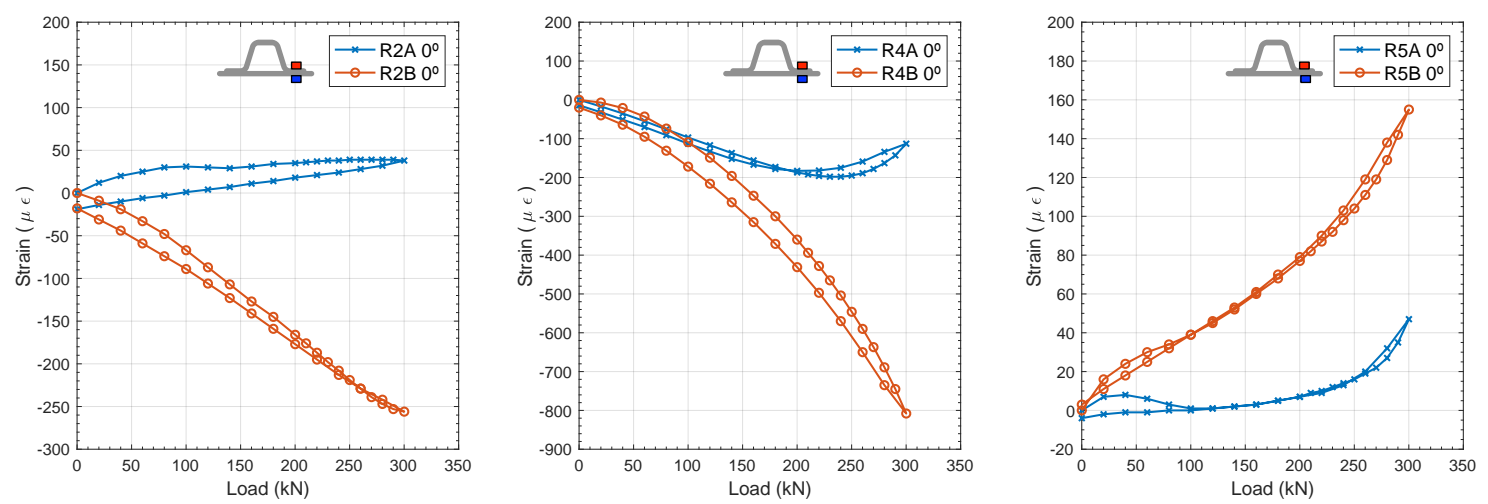

Figure 120. Rosettes strain gauge comparison at $0^{\circ}$ for buckling test under tensile load.

Summarising, the strain reading of the rosettes R2, R4 and R5 for both compressive and tensile test did not show the onset of local buckling at these locations. In addition, the rest of the rosettes could not be analysed due to installation issues throughout the buckling test. Regarding the strain comparison with the optical fibre, a good agreement was obtained, despite the fact that the exact location of the fibre was not bonded at the rosette location and the simulated strain was obtained through the linear interpolation of the neighbour's sensing points.

\subsubsection{Buckling detection by load-strain curves}

As formerly explained in 4.5.1, this methodology selects different random sensing points over the fibre optic length in order to study the load-strain response.

Figure 121 shows the application of this methodology on path 20. In this path, three different scenarios can be distinguished. First, at fibre length of 0.745 metres linear behaviour is recorded, and therefore the load-strain curve can be approximated to $y=-0.38 x-2.5$. Moreover, represented in the figure, the buckling behaviour appeared in the second half of the path but also, in a local area at the beginning of the path. Then, at the length of 0.290 metres under the highest loads, a local buckling can be appreciated. Furthermore, the buckling load started at $-210 \mathrm{kN}$. The same behaviour is encountered at fibre length of 1.115 
metres, which makes it even more noticeable. The strain readout sharply increases after the load of $-210 \mathrm{kN}$ due to the buckling appearance on a large length of the path.
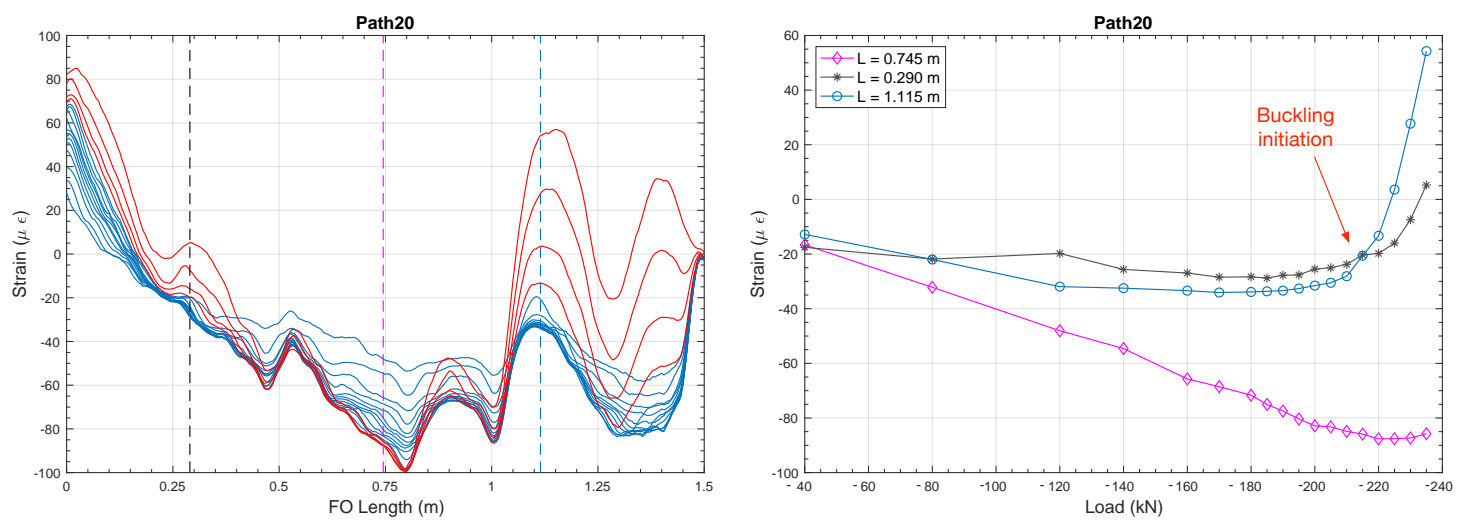

Figure 121. (Left) Distributed strain measurement recorded by the OBR for path 20 under compressive load. (Right) Bifurcation of load-strain curves at three different lengths.

The same methodology is applied on path 4 (Figure 122) to detect the buckling load. Despite the load strain level recorded in this path, due to its location at the foot of the stiffener, the buckling appearance can be clearly distinguished. Hence, linear behaviour was obtained in the middle of the path, at length $0.74 \mathrm{~m}$, with very small changes in the slope of the loadstrain curve. For the rest of the sensing points selected in the distributed measurement, the linearity of the load-strain curve is lost for a compressive load of around $-210 \mathrm{kN}$ as it occurred with path 20 .
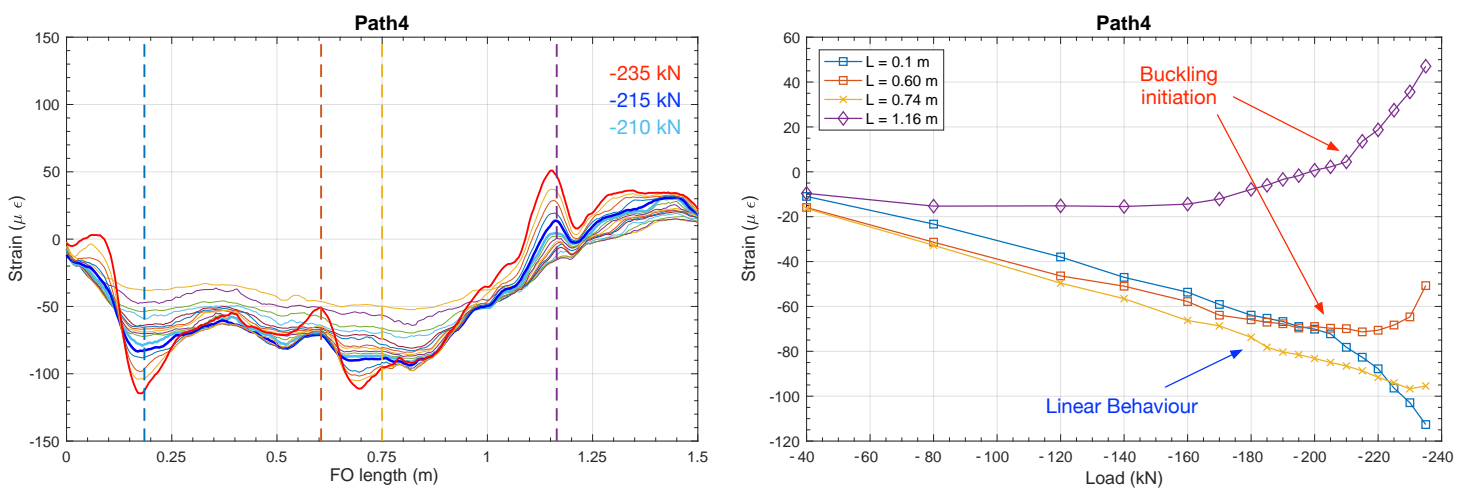

Figure 122. (Left) Distributed strain measurement recorded by the OBR for path 4 under compressive load. (Right) Bifurcation of load-strain curves at three different lengths.

Different behaviour is obtained on the same path under tensile loads. In this case, shown in Figure 123, linear behaviour is obtained at the different selected points. Only small variations can be observed along the load-strain curves. 

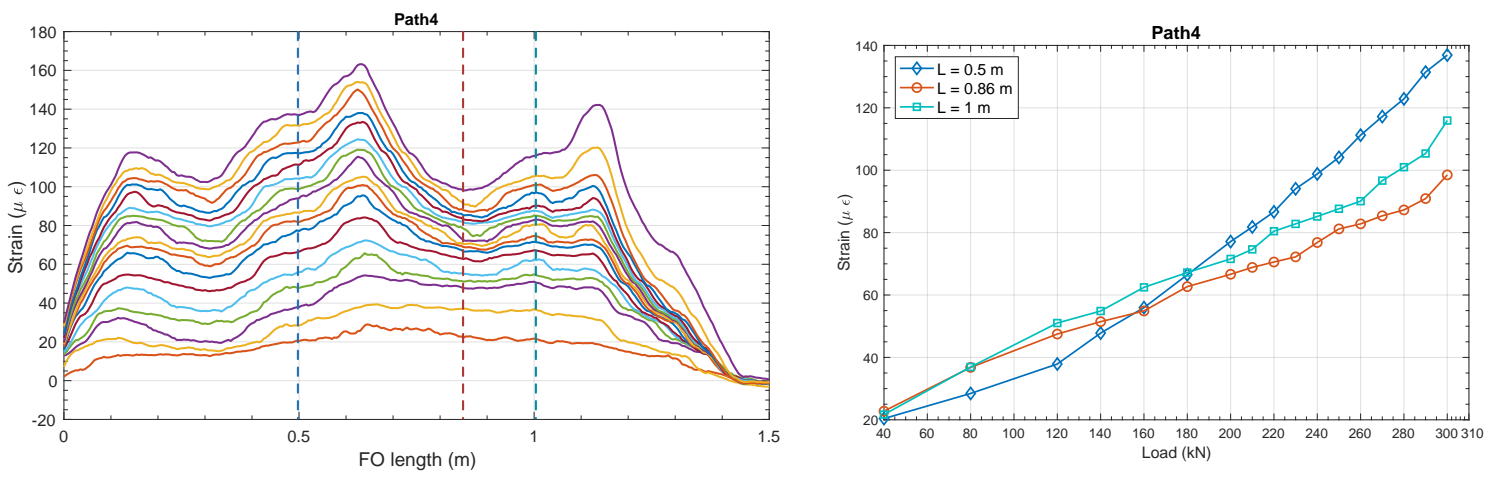

Figure 123. (Left) Distributed strain measurement recorded by the OBR for path 4 under tensile load. (Right) Bifurcation of load-strain curves at three different lengths.

This analysis can be performed at every sensing point which shapes the distributed measurement in order to detect the non-linear behaviour. The application of this methodology to detect the initiation of buckling is demonstrated not only for strain gauge measurement but also for distributed measurements. The main drawback of this methodology lies in the knowledge that the applied load needs to be known. Hence, the distributed strain measurement cannot act independently since it needs the load readout from another sensing system to detect the presence of non-linearities. Furthermore, in complex full-field strain the trend line has to be calculated for each sensing point to detect the deviation which is computationally expensive. However, this methodology is the most suitable to detect buckling in back-to-back strain gauge rosettes since three gauges are located on both sides of the panel and can be compared easily. The slope fluctuations of these curves are associated with non-linearities.

\subsubsection{Buckling detection by second derivative approach}

In Figure 124, the results of the compressive buckling tests are shown through the second derivative analysis. As can be appreciated, by means of the second derivative distributed map, different buckling waves can be distinguished over the panel at the last load step. The highest values of the second derivative were recorded on bay 9, 6 and 3 (upper bays of the figure). Then, the values on bay 6 and bay 3 were lower, but the apparition of the buckling waves can be still distinguished. Furthermore, it must be noted that the presence of the damaged area MUI1, also recorded the highest strain changes at this load step. This behaviour was expected since the delaminated area where this damage is located is the largest and it is prone to accumulate non-linear behaviour around it. Other local areas over the panel presented significant second derivative values as a consequence of the non-linear behaviour onset. 


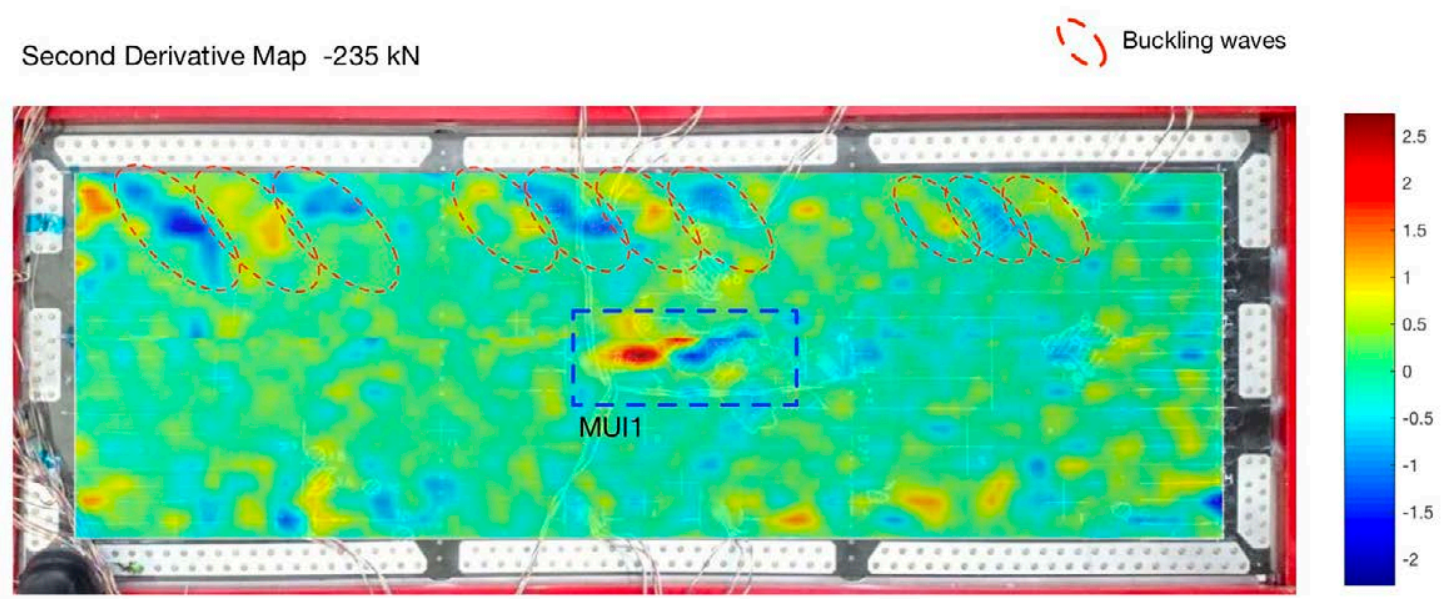

Figure 124. Results of the second derivative map for buckling detection under $-235 \mathrm{kN}$ of compressive load.

The paths which correspond to these bays are from path 2 to 5. As observed in Figure 125, the shape of the distributed strain measurement sharply changed all along the length of the optical fibre path, just as in the previous section, the bifurcation method was also analysed.
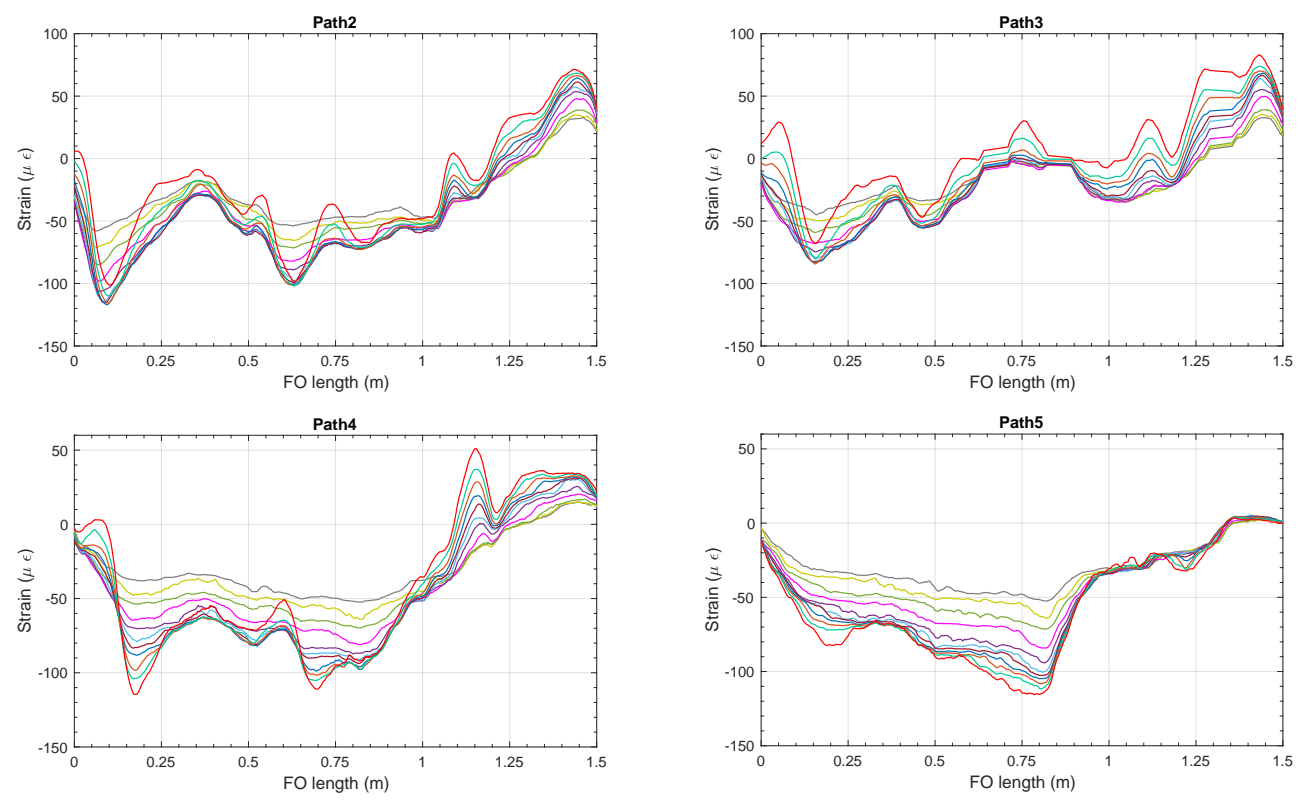

$-120 \mathrm{kN}--140 \mathrm{kN}=-160 \mathrm{kN}=-180 \mathrm{kN}=-200 \mathrm{kN}--210 \mathrm{kN}=-215 \mathrm{kN}=-220 \mathrm{kN}=-225 \mathrm{kN}--230 \mathrm{kN}=-235 \mathrm{kN}$

Figure 125. Distributed strain measurement for buckling test under compressive load on paths 2, 3,4 and 5 .

Different behaviour is obtained from the buckling test under tensile loads. In Figure 126, the second derivative map for $300 \mathrm{kN}$ of tensile load is displayed. A uniform second derivative 
field over whole surface of the stiffened panel can be appreciated. In the same manner that occurred in the buckling compressive test, other local areas presented non-linear behaviour..

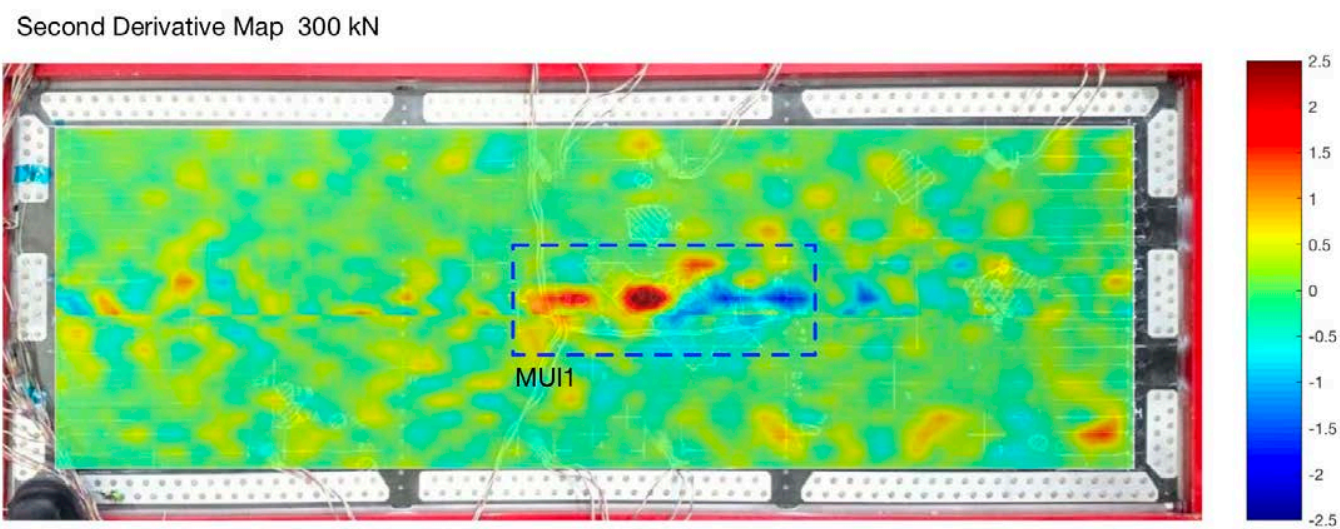

Figure 126. Results of the second derivative map for buckling detection under $300 \mathrm{kN}$ of tensile load.

Again, the MUI1 damaged area accumulated the highest second derivative values but, in this case, the affected area is larger than the one under compressive loads. This evidence is shown in Figure 127 through the path 11. Under compressive loads the strain level becomes more critical, but it is concentrated in a small area around the delamination. However, under tensile load, the strain level decreases and the strained area becomes extended.
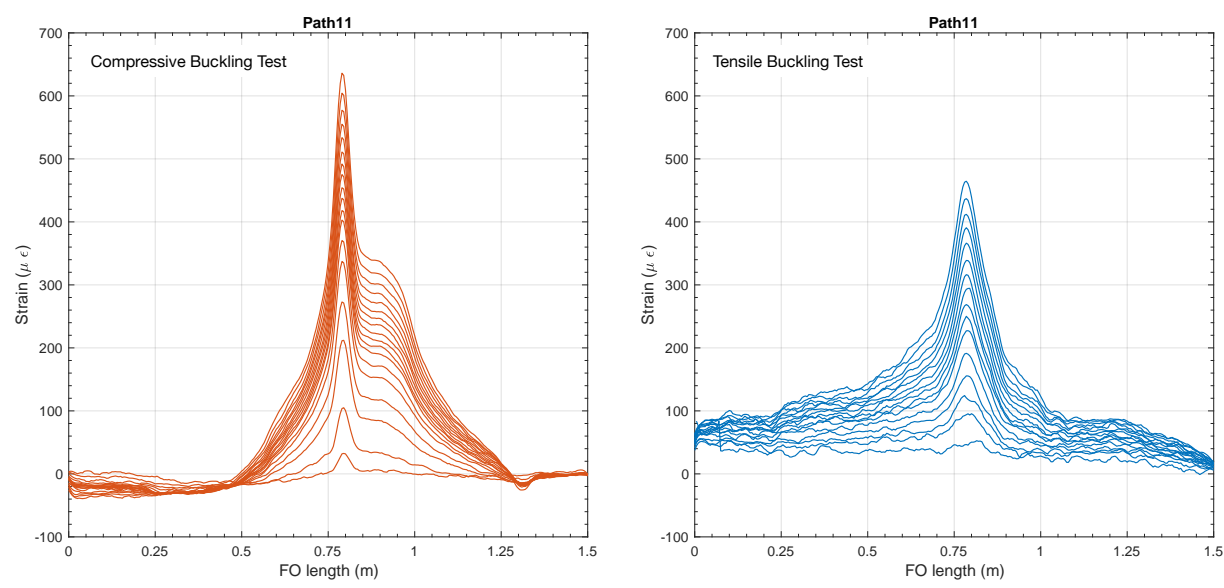

Figure 127. Comparison between compressive and tensile buckling test around damage MUI1.

\subsubsection{Conclusion of Panel 2}

Through this experimental test a full analysis has been carried out.

A detailed analysis of damage growth was performed regarding the results of the NDT results. It has been possible to compare the results of both techniques reaching a successful agreement. Only damaged areas with a small strain level present mixed results the same way 
that occurs with damage MUI4. In this case, the NDT detects no-growth of the damage but through the optical fibre a slight increase of the strain was recorded in the nearby area. Therefore, this increase is likely to be due to a small growth but not as critical as the other detected damages. The same case is obtained for damage MUI3. NDT detected three small but differentiated damage growths, but regarding the strain field no significant changes were obtained. In this case, the strain field hardly oscillated, thus, strain changes cannot be used to confirm the growth of the damage. For the rest of the damages, the agreement between the distributed measurement and the NDT can be well correlated. Moreover, the interval of the cycles from which the damage grew was also well identified and correlated in both techniques. In view of the results, distributed strain measurements can be proposed as an alternative technique to detect the growth of the damage by means of a simple acquisition of a distributed strain measurement. Regarding the conventional NDT methods that need a rigorous preparation to scan the stiffened panel skin damage by damage. This analysis turns into an overly expensive, time-consuming and manual handling procedure to detect the growth of the damage. This aspect will also be increased for structures installed in a real aircraft, which commonly need to be disassembled to carry out the NDT. For distributed strain measurements all of these aspects are solved. The interrogation of the optical fibre takes a few seconds and the comparison with the reference measurement is very straightforward. Moreover, the location of the fibre regarding the panel is previously known so as to identify the damaged area. However, an advantage of NDT over the fibre optic sensors is that the accurate position of the delamination damage can be well determined. A good approach to analyse the damage growth in a real aircraft structure could be by combining both techniques. Distributed strain measurement will be used as a first step to detect changes in the full-field strain and, once these changes exceed a limited threshold a deep NDT can be carried out providing as much detailed information as possible about the damage. In this way of working, important cost-savings in maintenance operations can be achieved.

Regarding the distributed measurement, a complete strain map of the stiffened panel could be obtained. The MUI1 recorded the highest strain level in both compressive and tensile tests. The results of the distributed measurements were not able to be compared with the rosette strain gauges due to most of them being unavailable after installation. However, rosettes 2, 4 and 5 were successfully compared with the distributed strain measurement. In this case, the survivability of fibre optic lines against conventional electrical strain gauges was demonstrated after carrying out all the experiments in the panel.

Again, the detection of buckling appearance could be predicted from the second derivative analysis of the distributed strain measurement. Not only the detection of the non-linear initiation but also the characterization of the buckling waves over the panel in a complex scenario of loads.

Regarding the strain field around the damages in the buckling test, what could be appreciated was that the delaminated area was larger under tensile loads but showed a strain gradient much more critical under compressive load. 


\subsection{Results Comparison}

In view of these results, distributed fibre optic sensing has been successfully used to analyse a whole fatigue test to detect the damage growth and also to monitor the buckling appearance on a stiffened panel under shear load induced by a diagonal compressive and tensile load.

Regarding the buckling appearance in both panels, a different location was obtained. Not only did the influence of the acoustic protection contribute significantly to the stiffened panel, but also the presence of the different damages. Specially for stiffened panel 2, where the centre bay was hugely affected by the delaminated area caused by MUI1 multi-impact. For the first panel the compressive buckling load appeared at $-185 \mathrm{kN}$ in agreement with the rosettes readings and the theoretical buckling load predicted by the FEM. As expected due to damages and specimen configuration, the stiffened panel buckled at $-210 \mathrm{kN}$ of compressive load which differs from the theoretical calculations. 


\section{Chapter 7 CONCLUSIONS AND FUTURE WORKS}

The work presented in this thesis addresses the structural health monitoring by distributed fibre optic sensors from a very practical point of view, considering local and global approaches.

In the first part of this thesis, the different distributed sensing techniques through fibre optics have been deeply described and analysed. Furthermore, a real experimental test was also presented by using an OTDR Raman system for temperature monitoring. A good agreement was obtained by comparing the temperature read-out of all available techniques: thermocouple, FBGs, DTS and OBR. Likewise, OFDR-FBG technique has been explored through the static test of a composite helicopter blade and compared with extensometers and FEM results. In line with FBG experiments, a pressurization test was monitored through a dense network of FBG over the composite cockpit of a regional aircraft. The difficulties to monitor large structures are here presented and evidenced when the FBG results were compared with those obtained for the strain gauges. The overall experimental tests carried out by the wide varied fibre optic techniques have enriched the development of the methodologies necessary to address the structural health monitoring by distributed strain measurement.

Prior to deal with the experimental part of this thesis, a theoretical background of distributed strain measurement acquired by measuring Rayleigh scattering is thoroughly analysed. In this part, the influence of the signal conditioning parameters is discussed since this choice strongly affects the resolution and accuracy of the distributed measurement. Learning the intrinsic principle of distributed measurement is a must to achieve reliable results of the performed experimental tests.

After presenting the damage detection methodology followed both local and global damage detection approaches, the results were exhibited and discussed. Moreover, particular conclusions were extracted and detailed after describing the results obtained for each test.

Main conclusions for the local approach obtained through the laboratory tests are summarised as follows:

- The good ability to embed a fibre optic into the adhesive layer of a double lap join in order to obtain a distributed strain measurement under loading conditions. However, the ingress/egress of fibre should be carefully positioned in order to 
avoid breakages which can spoil the measurement. Furthermore, the high temperature requirements due to adhesive layer curing is accomplished by using a special fibre coating of polyimide (thermal resistance up to $400^{\circ}$ ).

- Defects or design flaws was able to be captured by the distributed measurement when the optical was embedded, even if this defect is in the order of $1 \mathrm{~mm}$. Despite this, the strain level could not be reliable at the defect location, but its presence can be noted by high reflection peaks in the refractive pattern of the fibre.

- Good strain correlation was obtained when the optical fibre embedded is compared to analytical FEM. Furthermore, changes in the thickness were detectable when the laboratory specimen was loaded.

- Residual strains can be used to detect the presence of delamination areas, even when the released strain and the affected area was very small. Thus, it can be concluded that this approach could be especially useful on those structures which are prone to suffer accidental impacts. Therefore, the application of this technique to interrogate the presence of damage has been proven to be one of the most suitable regarding its fast and straightforward installation, non-intrusive and easy data post-processing from measurement acquisition. In addition, the location of the damages was accurately obtained as it was compared with the NDT results.

- Different configurations of optical fibre network can be installed over the different specimens in order to detect the delamination damage caused by low energy impacts.

Regarding the global approach through the distributed fibre optic sensors, main conclusions were extracted as described in the following:

- The ability to install a dense optical fibre was demonstrated in a complex and large scale experimental test. The survivability of the fibre was even proved after a fatigue test where the artificial damages over the structure grew. Conventional strain gauges had not succeeded the same results that the optical fibre network, since various sensors were unbonded throughout the same experimental test.

- The damage growth could be successfully monitored by the dense distributed measurements acquired at different fatigue cycles. Therefore, after comparing the strain readings at different load steps the residual full-field strain was obtained which contains valuable information regarding the released strain for each damage. 
- A novel methodology to detect the presence of buckling based on the analysis of the second derivative of the distributed strain measurement was developed and fruitfully proved. The buckling waves can be characterised and distinguished through the second distributed derivative map built from these measurements.

- A good correlation was obtained between the conventional methods for buckling detection such as the so-called "bifurcation" from the rosettes readings and the second derivative approach. Furthermore, a good agreement was also obtained for the buckling load detection through the different proposed techniques.

These points have summarized the main conclusions obtained through the presented experimental tests. As stated above, the distributed fibre optic sensors have been proposed in this thesis as a practical solution to be considered in the resolution of structural health monitoring issues. Therefore, through the presented work the capacity of this powerful technique has been explored and presented in a multifunctional manner.

As formerly explained in the OFDR chapter, distributed measurement techniques can be considered relatively recent in the FOS domain. And nowadays, DFOS Rayleigh-based is still an emerging technique in the race to achieve the best accuracy and resolution for distributed measurement by fibre optics. However, some goals are still on-going such as: achieving dynamic measurements without worsening the resolution, increasing the spatial range and the spatial resolution or isolating the strain from the temperature.

Finally, in the line of the work presented in this thesis, the following future works are enumerated as follows:

- Along the same lines, through the theoretical background presented in this thesis for OBR measurements (Chapter 3), the future works proposed lies in the autonomous selection of the gauge lengths for pre-process the binary data acquired by the OBR. This can be achieved by the development of an algorithm to adequate the sensor gauge to the external solicitation. Therefore, the signal condition parameter gauge length should not be fixed to obtain the distributed measurement. This goal can be obtained by maximizing the result of the cross correlation at every sensing point. In this thesis, the development of the algorithm has been performed but the exploitation of the data has not yet been implemented. All the results presented in Chapter 3 have been obtained with the mentioned algorithm.

- In view of the importance of the use of adhesives for aeronautical structures, further investigations of the growth of the artificial flaws introduced in the adhesive layer up is proposed. Being able to detect the failure of the adhesive layer through a single distributed strain measurement would help to increase the confidence in this mechanism to join different structural components. 
- The buckling detection of a stiffened composite panel under compressive loads have been detected, located and characterized by means of a fibre optic network bonded on the panel skin. Embed this dense network during the manufacturing process of the panel (Figure 10) is a challenge that can ensure a SHM system more robust. Then, analyse the capability to detect local and global buckling from these measurements, as well as the growth of the damages in comparison with the measurements obtained for the sensing network located on the flat side. Despite the fact that the fibre network will be much more protected when it is embedding from external events, such as accidental impacts, more information can be obtained. Hence, the presence of a delamination releasing very low energy as demonstrated through the impact on the free-edges, that can be detected by the optical fibre. Not only that, the fibre can be used to monitor the buckling appearance in real time but also after each flight cycle the residual strain can be used. 


\section{Bibliography}

Alonso García, J. (2016). Monitorización de estructuras aeronáuticas mediante técnicas de inteligencia artificial. Polytechnic University of Madrid.

Angulo-Vinuesa, X., Soto, M. a., Martin-Lopez, S., Chin, S., Ania-Castañon, J. D., Corredera, P., ... Thévenaz, L. (2012). Brillouin optical time-domain analysis over a $240 \mathrm{~km}-$ long fiber loop with no repeater. In Y. Liao, W. Jin, D. D. Sampson, R. Yamauchi, Y. Chung, K. Nakamura, \& Y. Rao (Eds.), (Vol. 8421, p. 8421C9-8421C9-4). http://doi.org/10.1117/12.2013201

Aydogdu, M., \& Aksencer, T. (2018). Buckling of cross-ply composite plates with linearly varying In-plane loads. Composite Structures, 183, 221-231. http://doi.org/10.1016/j.compstruct.2017.02.085

Bai, R., Lei, Z., Wei, X., Tao, W., \& Yan, C. (2017). Numerical and experimental study of dynamic buckling behavior of a J-stiffened composite panel under in-plane shear. Composite Structures, 166, 96-103. http://doi.org/10.1016/j.compstruct.2017.01.022

Balageas, D., Fritzen, C.-P., \& Güemes, A. (2006). Structural Health Monitoring. (D. Balageas, C.-P. Fritzen, \& A. Gemes, Eds.). London, UK: ISTE. http://doi.org/10.1002/9780470612071

Bao, X., \& Chen, L. (2012). Recent Progress in Distributed Fiber Optic Sensors. Sensors, 12(12), 8601-8639. http://doi.org/10.3390/s120708601

Barnoski, M. K., \& Jensen, S. M. (1976). Fiber waveguides: a novel technique for investigating attenuation characteristics. Applied Optics, 15(9), 2112. http://doi.org/10.1364/AO.15.002112

Barnoski, M. K., Rourke, M. D., Jensen, S. M., \& Melville, R. T. (1977). Optical time domain reflectometer. Applied Optics, 16(9), 2375. http://doi.org/10.1364/AO.16.002375

Bernini, R., Minardo, A., \& Zeni, L. (2012). Distributed Sensing at Centimeter-Scale Spatial Resolution by BOFDA: Measurements and Signal Processing. IEEE Photonics Journal, 4(1), 48-56. http://doi.org/10.1109/JPHOT.2011.2179024

Boller, C., \& Staszewski, W. J. (2004). Aircraft Structural Health and Usage Monitoring. In Health Monitoring of Aerospace Structures (pp. 29-73). Chichester, UK: John Wiley \& Sons, Ltd. http://doi.org/10.1002/0470092866.ch2

Bolognini, G., Park, J., Soto, M. A., Park, N., \& Di Pasquale, F. (2007). Analysis of distributed 
temperature sensing based on Raman scattering using OTDR coding and discrete Raman amplification. Measurement Science and Technology, 18(10), 3211-3218. http://doi.org/10.1088/0957-0233/18/10/S24

Box, G. E. P., Jenkins, G. M., \& Reinsel, G. C. (1994). Time Series Analysis: Forecasting and Control (3rd ed.). Englewood Cliffs, NJ: Prentice Hall. Retrieved from http://books.google.com.au/books?id=B8_1UBmqVUoC

Boyer, R., \& Padmapriya, N. (2016). Aircraft Materials. In Reference Module in Materials Science and Materials Engineering. Elsevier. http://doi.org/10.1016/B978-0-12-803581-8.01934-2

Brown, G., \& Hartog, A. (2002). Optical Fiber Sensors in Upstream Oil \&amp; Gas. Journal of Petroleum Technology, 54(11), 3. http://doi.org/10.2118/79080-MS

Butter, C. D., \& Hocker, G. B. (1978). Fiber optics strain gauge. Applied Optics, 17(18), 2867. http://doi.org/10.1364/AO.17.002867

Cao, M., Radzieński, M., Xu, W., \& Ostachowicz, W. (2014). Identification of multiple damage in beams based on robust curvature mode shapes. Mechanical Systems and Signal Processing, 46(2), 468-480. http://doi.org/10.1016/j.ymssp.2014.01.004

Carden, E. P., \& Fanning, P. (2004). Vibration based condition monitoring: A review. Structural Health Monitoring, 3(4), 355-377. http://doi.org/10.1177/1475921704047500

Castrellon-Uribe, J. (2012). Optical Fiber Sensors: An Overview. In Fiber Optic Sensors (pp. 126). InTech. http://doi.org/10.5772/28529

Chester, A. N., Martellucci, S., \& Scheggi, A. M. V. (1967). Optical Fiber Sensors. In NATO. ASI Series E: Applied Sciences No. 132. New York: Wiley \& Sons.

Clivaz, X., Marquis-Weible, F., \& Salathé, R. P. (1992). Optical low coherence reflectometry with $1.9 \mu \mathrm{m}$ spatial resolution. Electronics Letters, 28(16), 1553. http://doi.org/10.1049/el:19920986

Coburn, B. H., Wu, Z., \& Weaver, P. M. (2014). Buckling analysis of stiffened variable angle tow panels. Composite Structures, 111(1), 259-270. http://doi.org/10.1016/j.compstruct.2013.12.029

Cronkhite, J., Dickson, B., Martin, W., \& Collingwood, G. (1998). Operational Evaluation of a Health and Usage Monitoring System (HUMS). Fort Worth, Texas.

da Silva, L. F. M., Öchsner, A., \& Adams, R. D. (2011). Handbook of Adhesion Technology. (L. F. M. da Silva, A. Öchsner, \& R. D. Adams, Eds.). Berlin, Heidelberg: Springer Berlin Heidelberg. http://doi.org/10.1007/978-3-642-01169-6

Dakin, J. P., Pratt, D. J., Bibby, G. W., \& Ross, J. N. (1985). Distributed optical fibre Raman temperature sensor using a semiconductor light source and detector. Electronics Letters, 
21(13), 569. http://doi.org/10.1049/el:19850402

Denisov, A., Soto, M. A., \& Thévenaz, L. (2016). Going beyond 1000000 resolved points in a Brillouin distributed fiber sensor: Theoretical analysis and experimental demonstration. Light: Science and Applications, 5(October 2015). http://doi.org/10.1038/1sa.2016.74

Derickson, D. (1998). Fiber-Optic Test and Measurement. New Jersey: Prentice Hall.

Di Sante, R. (2015). Fibre Optic Sensors for Structural Health Monitoring of Aircraft Composite Structures: Recent Advances and Applications. Sensors, 15(12), 18666-18713. http://doi.org/10.3390/s150818666

Ding, Z., Wang, C., Liu, K., Jiang, J., Yang, D., Pan, G., ... Liu, T. (2018). Distributed Optical Fiber Sensors Based on Optical Frequency Domain Reflectometry: A review. Sensors, 18(4), 1072. http://doi.org/10.3390/s18041072

Donoho, D. L., \& Johnstone, J. M. (1994). Ideal spatial adaptation by wavelet shrinkage. Biometrika, 81(3), 425-455. http://doi.org/10.1093/biomet/81.3.425

Dutton, S., Kelly, D., \& Baker, A. (2004). Composite Materials for Aircraft Structures, Second Edition. Industrial Engineering (Vol. 43). Reston, VA: American Institute of Aeronautics and Astronautics. http://doi.org/10.2514/4.861680

Eickhoff, W., \& Ulrich, R. (1981). Optical frequency domain reflectometry in single-mode fiber. Applied Physics Letters, 39(9), 693-695. http://doi.org/10.1063/1.92872

F. Díaz-Maroto, P., Fernández-López, A., García-Alonso, J., Iglesias, M., \& Güemes, A. (2018). Buckling detection of an omega-stiffened aircraft composite panel using distributed fibre optic sensors. Thin-Walled Structures, 132, 375-384. http://doi.org/10.1016/j.tws.2018.08.024

F. Díaz-Maroto, P., Fernández, A., Fernández, R., Gutiérrez, N., Lasagni, F., \& Güemes, J. A. (2016). Strain monitoring on a composite aircraft cabin with fiber optic sensors. 8th European Workshop on Structural Health Monitoring, EWSHM 2016, 1, 186-194.

F. Díaz-Maroto, P., Fernández, A., Larrañaga, B., \& Guemes, A. (2016). Free-Edge delamination location and growth monitoring with an embedded distributed fiber optic network. In 8th European Workshop On Structural Health Monitoring. Bilbao, Spain.

F.Díaz-Maroto, P., Fernández-Lopez, A. ., \& Güemes, J. A. (2015). Distributed fibre optic sensors for stiffened skin panels strain monitoring and failure mode detection. In 10th International Workshop on Structural Health Monitoring. Stanford, CA, USA.

F.Díaz-maroto, P., López, A. F., \& Güemes, A. (2015). Monitorización de uniones adhesivas con sensores distribuidos de fibra óptica. In XI Congreso Nacional de Materiales Compuestos, MATCOMP15. Madrid. 
Fang, Z., Chin, K. K., Qu, R., \& Cai, H. (2012). Fundamentals of Optical Fiber Sensors. Fundamentals of Optical Fiber Sensors. Hoboken, NJ, USA: John Wiley \& Sons, Inc. http://doi.org/10.1002/9781118381717

Farrar, C. R., \& Worden, K. (2007). An introduction to structural health monitoring. Philosophical Transactions of the Royal Society A: Mathematical, Physical and Engineering Sciences, 365(1851), 303-315. http://doi.org/10.1098/rsta.2006.1928

Froggatt, M., \& Moore, J. (1998a). Distributed measurement of static strain in an optical fiber with multiple Bragg gratings at nominally equal wavelengths. Applied Optics, 37(10), 1741. http://doi.org/10.1364/AO.37.001741

Froggatt, M., \& Moore, J. (1998b). High-spatial-resolution distributed strain measurement in optical fiber with Rayleigh scatter. Applied Optics, 37(10), 1735. http://doi.org/10.1364/AO.37.001735

Fugate, M. L., Sohn, H., \& Farrar, C. R. (2001). Vibration-Based Damage Detection Using Statistical Process Control. Mechanical Systems and Signal Processing, 15(4), 707-721. http://doi.org/10.1006/mssp.2000.1323

Garcus, D., Gogolla, T., Krebber, K., \& Schliep, F. (1997). Brillouin optical-fiber frequencydomain analysis for distributed temperature and strain measurements. Journal of Lightwave Technology, 15(4), 654-662. http://doi.org/10.1109/50.566687

Gay, D. (2015). Composite Materials: Design and Applications (Third Edit). Boca Raton, FL: CRC Press, Taylor \& Francis.

Gentile, A., \& Messina, A. (2003). On the continuous wavelet transforms applied to discrete vibrational data for detecting open cracks in damaged beams. International Journal of Solids and Structures, 40(2), 295-315. http://doi.org/10.1016/S0020-7683(02)00548-6

Gifford, D. K. (2005). Distributed fiber-optic temperature sensing using Rayleigh backscatter. 31st European Conference on Optical Communications ECOC 2005, 2005(1), v3-511-v3-511. http://doi.org/10.1049/cp:20050584

Glisic, B. (2013). Distributed Fiber Optic Sensing Technologies and Applications - An Overview. Special Publication, 292. http://doi.org/10.14359/51686284

Glišić, B., \& Inaudi, D. (2007). Fibre Optic Methods for Structural Health Monitoring. Chichester, UK: John Wiley \& Sons, Ltd. http://doi.org/10.1002/9780470517819

Gonzalez-Chi, P. I., Flores-Johnson, E. A., Carrillo-Baeza, J. G., \& Young, R. J. (2010). Micromechanical analysis of the kink-band performance at the interface of a thermoplastic composite under tensile deformation. Polymer Composites, 31(10), 18171821. http://doi.org/10.1002/pc.20973

Grattan, K. T. V., \& Ning, Y. N. (1998). Classification of optical fiber sensors. In Optical Fiber 
Sensor Technology (pp. 1-35). Boston, MA: Springer US. http://doi.org/10.1007/978-14615-5787-6_1

Grave, J. H. L., Håheim, M. L., \& Echtermeyer, A. T. (2015). Measuring changing strain fields in composites with Distributed Fiber-Optic Sensing using the optical backscatter reflectometer. Composites Part B: Engineering, 74, 138-146. http://doi.org/10.1016/j.compositesb.2015.01.003

Güemes, A., \& Fernandez-Lopez, A. (2010). Damage Detection in Bolted Joints by Fibre Optics Distributed Sensing. In 2nd International Symposium on NDT in Aerospace.

Güemes, A., Fernández-López, A., Díaz-Maroto, P., Lozano, A., \& Sierra-Perez, J. (2018). Structural Health Monitoring in Composite Structures by Fiber-Optic Sensors. Sensors, 18(4), 1094. http://doi.org/10.3390/s18041094

Guemes, A., Fernandez-Lopez, A., \& Soller, B. (2010). Optical fiber distributed sensing physical principles and applications. Structural Health Monitoring, 9(3), 233-245. http://doi.org/10.1177/1475921710365263

Guillamet, G., Turon, A., Costa, J., \& Linde, P. (2016). A quick procedure to predict free-edge delamination in thin-ply laminates under tension. Engineering Fracture Mechanics, 168, 28-39. http://doi.org/10.1016/j.engfracmech.2016.01.019

Gyger, F., Rochat, E., Chin, S., Niklès, M., \& Thévenaz, L. (2014). Extending the sensing range of Brillouin optical time-domain analysis up to $325 \mathrm{~km}$ combining four optical repeaters. In J. M. López-Higuera, J. D. C. Jones, M. López-Amo, \& J. L. Santos (Eds.), 23rd International Conference on Optical Fibre Sensors (Vol. 9157, p. 91576Q). http://doi.org/10.1117/12.2059590

Harris, D. C., \& Bertolucci, M. D. (1978). Symmetry and Spectroscopy: Introduction to Vibrational and Electronic Spectroscopy. New York, NY Dover 2014: Dover Publications, Inc. Retrieved from http://www.worldcat.org/title/symmetry-and-spectroscopy-anintroduction-to-vibrational-and-electronic-spectroscopy/oclc/935923615

He, J., Zhou, Z., \& Ou, J. (2014). Simultaneous measurement of strain and temperature using a hybrid local and distributed optical fiber sensing system. Measurement, 47, 698-706. http://doi.org/10.1016/j.measurement.2013.10.006

Hill, K. O., Fujii, Y., Johnson, D. C., \& Kawasaki, B. S. (1978). Photosensitivity in optical fiber waveguides: Application to reflection filter fabrication. Applied Physics Letters, 32(10), 647-649. http://doi.org/10.1063/1.89881

Hill, K. O., Malo, B., Bilodeau, F., Johnson, D. C., \& Albert, J. (1993). Bragg gratings fabricated in monomode photosensitive optical fiber by UV exposure through a phase mask. Applied Physics Letters, 62(10), 1035-1037. http://doi.org/10.1063/1.108786 
Hill, K. O., Malo, B., Vineberg, K. A., Bilodeau, F., Johnson, D. C., \& Skinner, I. (1990). Efficient mode conversion in telecommunication fibre using externally written gratings. Electronics Letters, 26(16), 1270. http://doi.org/10.1049/el:19900818

Hill, K. O., \& Meltz, G. (1997). Fiber Bragg grating technology fundamentals and overview. Journal of Lightwave Technology, 15(8), 1263-1276. http://doi.org/10.1109/50.618320

Himelblau, H., Manning, J., Kern, D., \& Piersol, A. (2001). Dynamic Environmental Criteria. NASA Technical Handbook (Vol. HDBK-7005). Retrieved from http://scholar.google.com/scholar?hl=en\&btnG=Search\&q=intitle:DYNAMIC+ENVIRO NMENTAL+CRITERIA\#0

Hocheng, H., \& Tsao, C. . (2003). Comprehensive analysis of delamination in drilling of composite materials with various drill bits. Journal of Materials Processing Technology, 140(1-3), 335-339. http://doi.org/10.1016/S0924-0136(03)00749-0

Hocker, G. B. (1979). Fiber-optic sensing of pressure and temperature. Applied Optics, 18(9), 1445. http://doi.org/10.1364/AO.18.001445

Hoff N.J., Boley B.A., C. J. M. (1948). The development of a technique for testing stiff panels in edgewise compression. In Proceedings of the Society for Experimental Stress Analysis.

Horiguchi, T., Kurashima, T., \& Tateda, M. (1989). Tensile strain dependence of Brillouin frequency shift in silica optical fibers. IEEE Photonics Technology Letters, 1(5), 107-108. http://doi.org/10.1109/68.34756

Horiguchi, T., Shimizu, K., Kurashima, T., Tateda, M., \& Koyamada, Y. (1995). Development of a distributed sensing technique using Brillouin scattering. Journal of Lightwave Technology, 13(7), 1296-1302. http://doi.org/10.1109/50.400684

Hotate, K., \& Hasegawa, T. (2000). Measurement of Brillouin Gain Spectrum Distribution along an Optical Fiber Using a Correlation-Based Technique -- Proposal, Experiment and Simulation. IEICE Trans. Electronics, E83-C(3), 405-412. http://doi.org/10.1.1.29.7344

Hurtig, E., Großwig, S., \& Kühn, K. (1996). Fibre optic temperature sensing: application for subsurface and ground temperature measurements. Tectonophysics, 257(1), 101-109. http://doi.org/10.1016/0040-1951(95)00124-7

Igawa, H., Murayama, H., Kasai, T., Yamaguchi, I., Kageyama, K., \& Ohta, K. (2005). Measurements of strain distributions with a long gauge FBG sensor using optical frequency domain reflectometry. In Test (Vol. 5855, p. 547). http://doi.org/10.1117/12.624250

Igawa, H., Ohta, K., Kasai, T., Yamaguchi, I., Murayama, H., \& Kageyama, K. (2008). Distributed Measurements with a Long Gauge FBG Sensor Using Optical Frequency Domain Reflectometry. Journal of Solid Mechanics and Materials Engineering, 2(9), 1242- 
1252. http://doi.org/10.1299/jmmp.2.1242

Ishii, H., Kawamura, K., Ono, T., Megumi, H., \& Kikkawa, A. (1997). A fire detection system using optical fibres for utility tunnels. Fire Safety Journal, 29(2-3), 87-98. http://doi.org/10.1016/S0379-7112(96)00065-3

Jiang, X., Ma, Z. J., \& Ren, W.-X. (2012). Crack Detection from the Slope of the Mode Shape Using Complex Continuous Wavelet Transform. Computer-Aided Civil and Infrastructure Engineering, 27(3), 187-201. http://doi.org/10.1111/j.1467-8667.2011.00734.x

Jin, T. L., Ha, N. S., \& Goo, N. S. (2014). A study of the thermal buckling behavior of a circular aluminum plate using the digital image correlation technique and finite element analysis. Thin-Walled Structures, 77, 187-197. http://doi.org/10.1016/j.tws.2013.10.012

Kapron, F. P., Maurer, R. D., \& Teter, M. P. (1972). Theory of Backscattering Effects in Waveguides. Applied Optics, 11(6), 1352. http://doi.org/10.1364/AO.11.001352

Kashyap, R. (2010). Fiber Bragg Gratings. Fiber Bragg Gratings. Elsevier. http://doi.org/10.1016/C2009-0-16830-7

Kassapoglou, C. (2013). Design and Analysis of Composite Structures. John Wiley E Sons (First). Oxford, UK: John Wiley \& Sons Ltd. http://doi.org/10.1002/9781118536933

Kawasaki, B. S., Hill, K. O., Johnson, D. C., \& Fujii, Y. (1978). Narrow-band Bragg reflectors in optical fibers. Optics Letters, 3(2), 66. http://doi.org/10.1364/OL.3.000066

Kersey, A. D., Davis, M. A., Patrick, H. J., LeBlanc, M., Koo, K. P., Askins, C. G., ... Friebele, E. J. (1997). Fiber grating sensors. Journal of Lightwave Technology, 15(8), 1442-1463. http://doi.org/10.1109/50.618377

Kersey, A. D., Marrone, M. J., \& Davis, M. A. (1991). Polarisation-insensitive fibre optic Michelson interferometer. Electronics Letters, 27(6), 518. http://doi.org/10.1049/el:19910325

Kersey, A. D., \& Morey, W. W. (1993). Multi-element Bragg-grating based fibre-laser strain sensor. Electronics Letters, 29(11), 964-966. http://doi.org/10.1049/el:19930642

Krohn, D., MacDougall, T., \& Mendez, Al. (2014). Fiber Optic Sensor Funddamentals and Applications.

Kurashima, T., Horiguchi, T., Izumita, H., Furukawa, S. ichi, \& Koyamada, Y. (1993). Brillouin optical-fiber time domain reflectometry. IEICE Transactions on Communications, E76-B(4), 382-390.

Kurashima, T., Horiguchi, T., \& Tateda, M. (1990). Distributed-temperature sensing using stimulated Brillouin scattering in optical silica fibers. Optics Letters, 15(18), 1038. http://doi.org/10.1364/OL.15.001038 
Lagakos, N., Bucaro, J. A., \& Hughes, R. (1980). Acoustic sensitivity predictions of singlemode optical fibers using Brillouin scattering. Applied Optics, 19(21), 3668.

http://doi.org/10.1364/AO.19.003668

Lesiak, P., Szeląg, M., Budaszewski, D., Plaga, R., Mileńko, K., Rajan, G., ... Woliński, T. (2012). Influence of lamination process on optical fiber sensors embedded in composite material. Measurement, 45(9), 2275-2280.

http://doi.org/10.1016/j.measurement.2012.03.010

Leung, C. K. Y., Wan, K. T., Inaudi, D., Bao, X., Habel, W., Zhou, Z., ... Imai, M. (2015). Review: optical fiber sensors for civil engineering applications. Materials and Structures, 48(4), 871-906. http://doi.org/10.1617/s11527-013-0201-7

Liu, Y., Peng, W., Liang, Y., Zhang, X., Zhou, X., \& Pan, L. (2013). Fiber-optic Mach-Zehnder interferometric sensor for high-sensitivity high temperature measurement. Optics Communications, 300, 194-198. http://doi.org/10.1016/j.optcom.2013.02.054

London, Y., Antman, Y., Preter, E., Levanon, N., \& Zadok, A. (2016). Brillouin Optical Correlation Domain Analysis Addressing 440000 Resolution Points. Journal of Lightwave Technology, 34(19), 4421-4429. http://doi.org/10.1109/JLT.2016.2521173

Long, S., Yao, X., \& Zhang, X. (2015). Delamination prediction in composite laminates under low-velocity impact. Composite Structures, 132, 290-298.

http://doi.org/10.1016/j.compstruct.2015.05.037

Luna Technologies Inc. (2013). Optical Backscatter Reflectometer 4600 User Guide. Luna Technologies. Blacksburg, VA, USA.

Luna Technologies Inc. (2014). Optical Backscatter Reflectometer Length Measurement Accuracy. Retrieved from http://lunainc.com/wpcontent/uploads/2015/02/LT_TD_ENFY14-06_OBR_LengthMsrmt_Rev1.pdf

Lyon, R. E., Chike, K. E., \& Angel, S. M. (1994). In situ cure monitoring of epoxy resins using fiber-optic Raman spectroscopy. Journal of Applied Polymer Science, 53(13), 1805-1812. http://doi.org/10.1002/app.1994.070531310

MacDonald, R. (1981). Frequency domain optical reflectometer. Applied Optics, 20(10), 18401844. Retrieved from http://www.opticsinfobase.org/ao/fulltext.cfm?uri=ao-20-101840\&id=24908

Malhotra, A., \& Guild, F. J. (2014). Impact Damage to Composite Laminates: Effect of Impact Location. Applied Composite Materials, 21(1), 165-177. http://doi.org/10.1007/s10443-0139382-z

Malhotra, A., Guild, F. J., \& Pavier, M. J. (2008). Edge impact to composite laminates: experiments and simulations. Journal of Materials Science, 43(20), 6661-6667. 
http://doi.org/10.1007/s10853-008-2863-z

Mallela, U. K., \& Upadhyay, A. (2006). Buckling of laminated composite stiffened panels subjected to in-plane shear: A parametric study. Thin-Walled Structures, 44(3), 354-361. http://doi.org/10.1016/j.tws.2006.03.008

Maurer, R. D., \& Schultz, P. C. (1972). Fused Silica Optical Waveguide. United States.

Meltz, G., Morey, W. W., \& Glenn, W. H. (1989). Formation of Bragg gratings in optical fibers by a transverse holographic method. Optics Letters, 14(15), 823. http://doi.org/10.1364/OL.14.000823

Menadier, C., Kissinger, C., \& Adkins, H. (1967). The fotonic sensor. Instruments and Control Systems, 40, 114-120.

Menéndez, J. M. (1999). Redes de difracción de bragg como sensores de deformación. Polytechnic University of Madrid.

Minakuchi, S., Okabe, Y., \& Takeda, N. (2007). Barely visible impact damage detection in sandwich structures using non-uniform strain along optical fiber sensors. 16th International Conference On Composite Materials, 1-7.

Murayama, H., Kageyama, K., Naruse, H., Shimada, A., \& Uzawa, K. (2003). Application of Fiber-Optic Distributed Sensors to Health Monitoring for Full-Scale Composite Structures. Journal of Intelligent Material Systems and Structures, 14(1), 3-13. http://doi.org/10.1177/1045389X03014001001

Murayama, H., Wada, D., \& Igawa, H. (2013). Structural health monitoring by using fiberoptic distributed strain sensors with high spatial resolution. Photonic Sensors, 3(4), 355376. http://doi.org/10.1007/s13320-013-0140-5

Ortiz de Mendibil, I., F. Díaz-Maroto, P., Fernández-Lopez, A., Aurrekoetxea, J., \& Güemes, A. (2014). ${ }^{\circ}$. In The 12th International Conference on Flow Processing in Composite Materials. Enschede, NETHERLANDS.

Ostachowicz, W., \& Güemes, J. A. (2013). New Trends in Structural Health Monitoring. (W. Ostachowicz \& J. A. Güemes, Eds.) (Vol. 542). Vienna: Springer Vienna. http://doi.org/10.1007/978-3-7091-1390-5

Ostachowicz, W., Malinowski, P., \& Wandowski, T. (2013). Damage localisation using elastic waves propagation method. Experimental techniques. In New Trends in Structural Health Monitoring (pp. 317-371). http://doi.org/10.1007/978-3-7091-1390-5_6

Pandey, A. K., \& Biswas, M. (1994). Damage Detection in Structures Using Changes in Flexibility. Journal of Sound and Vibration, 169(1), 3-17. http://doi.org/10.1006/jsvi.1994.1002 
Pandey, A. K., Biswas, M., \& Samman, M. M. (1991). Damage detection from changes in curvature mode shapes. Journal of Sound and Vibration, 145(2), 321-332. http://doi.org/10.1016/0022-460X(91)90595-B

Passy, R., Gisin, N., von der Weid, J. P., \& Gilgen, H. H. (1994). Experimental and theoretical investigations of coherent OFDR with semiconductor laser sources. Journal of Lightwave Technology, 12(9), 1622-1630. http://doi.org/10.1109/50.320946

Pluciński, J., Hypszer, R., Wierzba, P., Strąkowski, M., Maciejewski, M., \& Kosmowski, B. B. (2008). Optical low-coherence interferometry for selected technical applications. Bulletin of the Polish Academy of Sciences Technical, 56(2), 155-172.

Powell, J. A. (1974). A simple two-fiber optical displacement sensor. Review of Scientific Instruments, 45(2), 302-303. http://doi.org/10.1063/1.1686613

Rao, Y.-J. (1997). In-fibre Bragg grating sensors. Measurement Science and Technology, 8(4), 355375. http://doi.org/10.1088/0957-0233/8/4/002

Rich, T. C., \& Pinnow, D. a. (1974). Evaluation of fiber optical waveguides using brillouin spectroscopy. Applied Optics, 13(6), 1376-1378. http://doi.org/10.1364/AO.13.001376

Rogers, A. (1999). Distributed optical-fibre sensing. Measurement Science and Technology, 10(8), R75-R99. http://doi.org/10.1088/0957-0233/10/8/201

Roy, K. (2017). Structural damage identification using mode shape slope and curvature. J. Eng. Mech., 143(9), 1-12. http://doi.org/10.1061/(ASCE)EM.1943-7889.0001305.

Rytter, A. (1993). Vibrational Based Inspection of Civil Engineering Structures. University of Aalborg, Denmark.

Sang, A. K., Froggatt, M. E., Gifford, D. K., Kreger, S. T., \& Dickerson, B. D. (2008). One Centimeter Spatial Resolution Temperature Measurements in a Nuclear Reactor Using Rayleigh Scatter in Optical Fiber. IEEE Sensors Journal, 8(7), 1375-1380. http://doi.org/10.1109/JSEN.2008.927247

Santos, J. L., \& Farahi, F. (2015). Handbook of Optical Sensors. Polymeric Sensors and Actuators. http://doi.org/10.1002/9781118547663

Savitzky, A., \& Golay, M. J. E. (1964). Smoothing and Differentiation of Data by Simplified Least Squares Procedures. Analytical Chemistry, 36(8), 1627-1639. http://doi.org/10.1021/ac60214a047

Schenato, L. (2017). A Review of Distributed Fibre Optic Sensors for Geo-Hydrological Applications. Applied Sciences, 7(12), 896. http://doi.org/10.3390/app7090896

Shimizu, T., Yari, T., Nagai, K., \& Takeda, N. (2001). Strain measurement using a Brillouin optical time domain reflectometer for development of aircraft structure health 
monitoring system. In T. Kundu (Ed.), 6th Annual International Symposium on NDE for Health Monitoring and Diagnostics (Vol. 4335, p. 312). Newport Beach, CA, United States. http://doi.org/10.1117/12.434187

Soller, B. J., Gifford, D. K., Wolfe, M. S., \& Froggatt, M. E. (2005). High resolution optical frequency domain reflectometry for characterization of components and assemblies. Optics Express, 13(2), 666. http://doi.org/10.1364/OPEX.13.000666

Soller, B. J., Wolfe, M. S., \& Froggatt, M. E. (2005). Polarization resolved measurement of Rayleigh backscatter in fiber-optic components. OFC Technical Digest, Los Angeles, (March), 1-6.

Song, J., Li, W., Lu, P., Xu, Y., Chen, L., \& Bao, X. (2014). Long-Range High Spatial Resolution Distributed Temperature and Strain Sensing Based on Optical Frequency-Domain Reflectometry. IEEE Photonics Journal, 6(3). http://doi.org/10.1109/JPHOT.2014.2320742

Sorin, W. V., \& Baney, D. M. (1992). Measurement of Rayleigh backscattering at $1.55 \mathrm{mu} \mathrm{m}$ with 32 mu m spatial resolution. IEEE Photonics Technology Letters, 4(4), 374-376. http://doi.org/10.1109/68.127218

Sorin, W. V. (1993). High-resolution optical fiber reflectometry techniques. In J. P. Dakin \& A. D. Kersey (Eds.), Proceedings of the SPIE The International Society for Optical Engineering (Vol. 1797, pp. 109-118). http://doi.org/10.1117/12.141281

Takeda, N., Minakuchi, S., \& Nadabe, T. (2013). Distributed Strain Monitoring for Damage Evolution in CFRP Bolted Structures with Embedded Optical Fibers. Key Engineering Materials, 558, 252-259. http://doi.org/10.4028/www.scientific.net/KEM.558.252

Tang, C. L. (1966). Saturation and Spectral Characteristics of the Stokes Emission in the Stimulated Brillouin Process. Journal of Applied Physics, 37(8), 2945-2955. http://doi.org/10.1063/1.1703144

Timoshenko, S. P., \& Goodier, J. N. (1951). Theory of Elasticity. New York: McGraw-Hill. http://doi.org/10.1017/S036839310012471X

Tkach, Chraplyvy, \& Derosier. (1986). Spontaneous brillouin scattering for single mode optical fiber characterization. Electronics Letters, 22(19), 1011-1013. http://doi.org/10.1049/el:19860691

Todd, M. D. (2005). Optical-Based Sensing. In Damage Prognosis (pp. 343-362). Chichester, UK: John Wiley \& Sons, Ltd. http://doi.org/10.1002/0470869097.ch17

Trutzel, M. N., Wauer, K., Betz, D., Staudigel, L., Krumpholz, O., Muehlmann, H.-C., ... Gleine, W. (2000). Smart Sensing of Aviation Structures with Fiber-optic Bragg Grating Sensors. Proc. SPIE 3986, Smart Structures and Materials 2000: Sensory Phenomena and Measurement Instrumentation for Smart Structures and Materials, 3986, 134-143. 
http://doi.org/10.1117/12.388099

von der Weid, J. P., Passy, R., Mussi, G., \& Gisin, N. (1997). On the characterization of optical fiber network components with optical frequency domain reflectometry. Journal of Lightwave Technology, 15(7), 1131-1141. http://doi.org/10.1109/50.596958

Vurpillot, S., Inaudi, D., \& Ducret, J.-M. (1996). Bridge monitoring by fiber optic deformation sensors: design, emplacement, and results. In L. K. Matthews (Ed.), Smart Structures and Materials 1996: Smart Systems for Bridges, Structures, and Highways (Vol. 2719, pp. 141149). http://doi.org/10.1117/12.238833

Wada, D., Igawa, H., Murayama, H., \& Kasai, T. (2014). Signal processing method based on group delay calculation for distributed Bragg wavelength shift in optical frequency domain reflectometry. Optics Express, 22(6), 6829. http://doi.org/10.1364/OE.22.006829

Waite, S. R., \& Sage, G. N. (1988). The failure of optical fibres embedded in composite materials. Composites, 19(4), 288-294. http://doi.org/10.1016/0010-4361(88)90005-5

Worden, K., \& Dulieu-Barton, J. M. (2004). An Overview of Intelligent Fault Detection in Systems and Structures. Structural Health Monitoring: An International Journal, 3(1), 85-98. http://doi.org/10.1177/1475921704041866

Xin, H., Mosallam, A., Liu, Y., Xiao, Y., He, J., Wang, C., \& Jiang, Z. (2017). Experimental and numerical investigation on in-plane compression and shear performance of a pultruded GFRP composite bridge deck. Composite Structures, 180, 914-932. http://doi.org/10.1016/j.compstruct.2017.08.066

Youngquist, R. C., Carr, S., \& Davies, D. E. N. (1987). Optical coherence-domain reflectometry: a new optical evaluation technique. Optics Letters, 12(3), 158. http://doi.org/10.1364/OL.12.000158

Zhu, S., Yan, J., Chen, Z., Tong, M., \& Wang, Y. (2015). Effect of the stiffener stiffness on the buckling and post-buckling behavior of stiffened composite panels - Experimental investigation. Composite Structures, 120, 334-345. http://doi.org/10.1016/j.compstruct.2014.10.021 


\section{Appendix}

This appendix contains all results of the chapter global damage detection.

A. Results of buckling analysis by second derivative for the stiffened panel 1 .
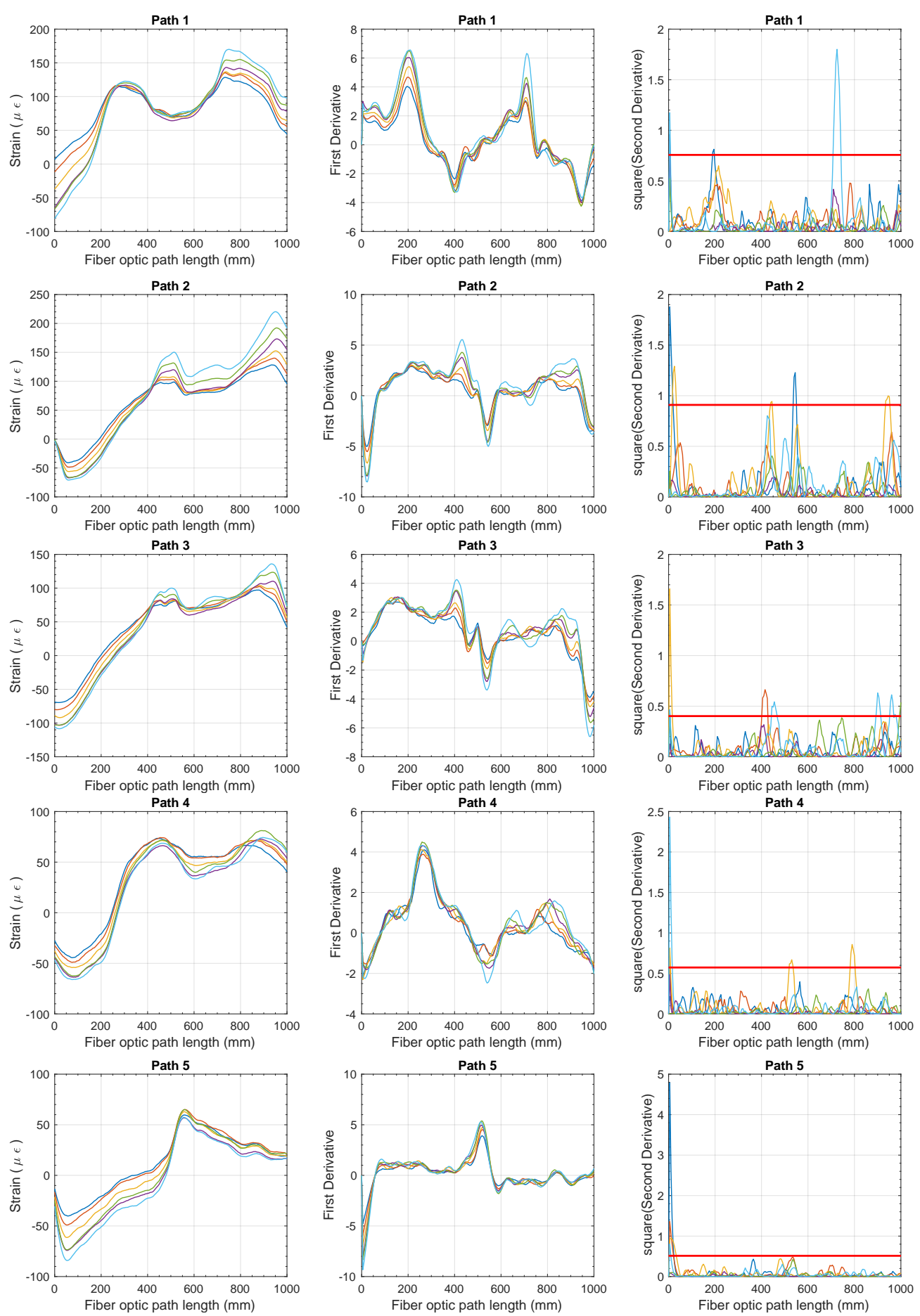

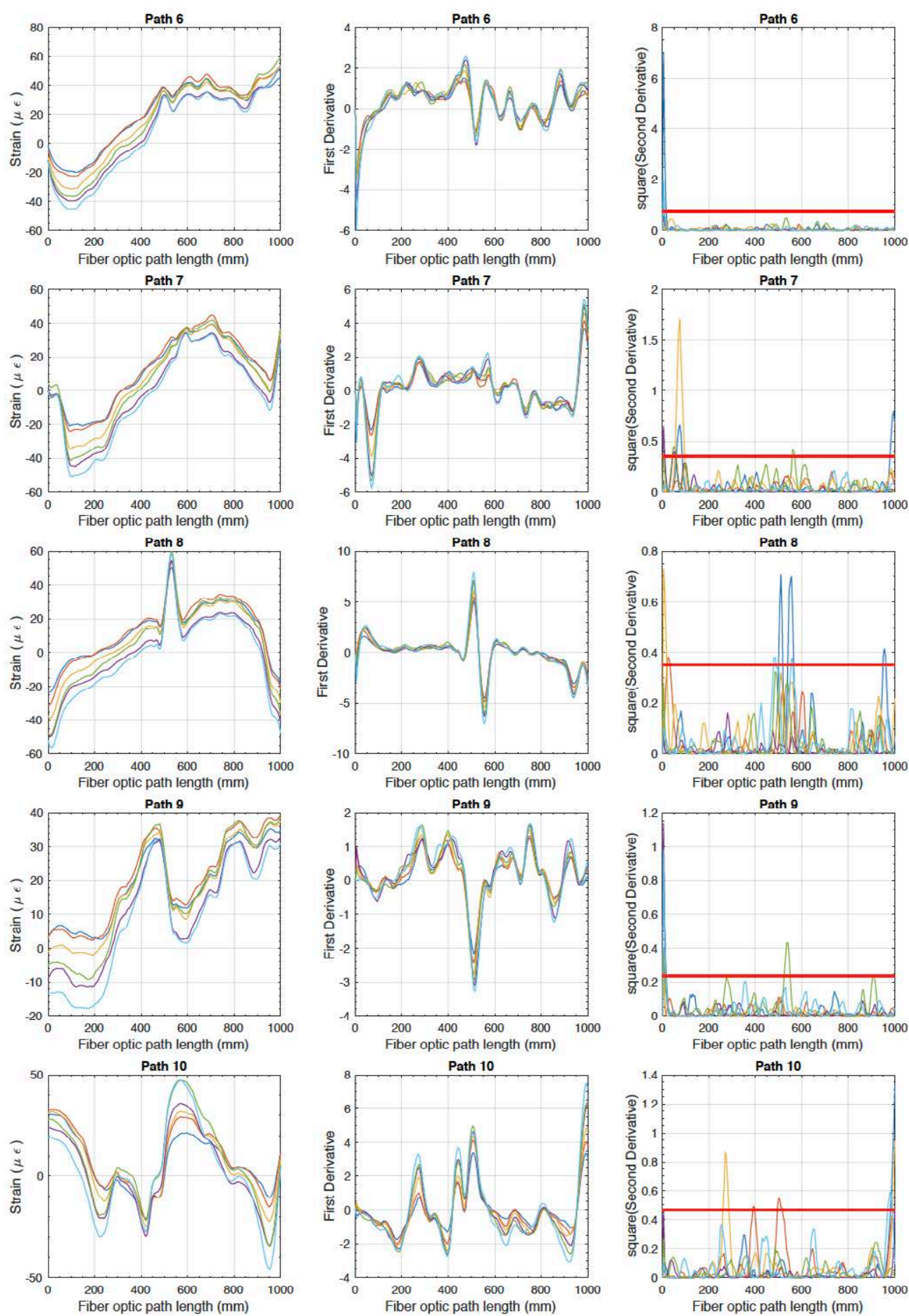

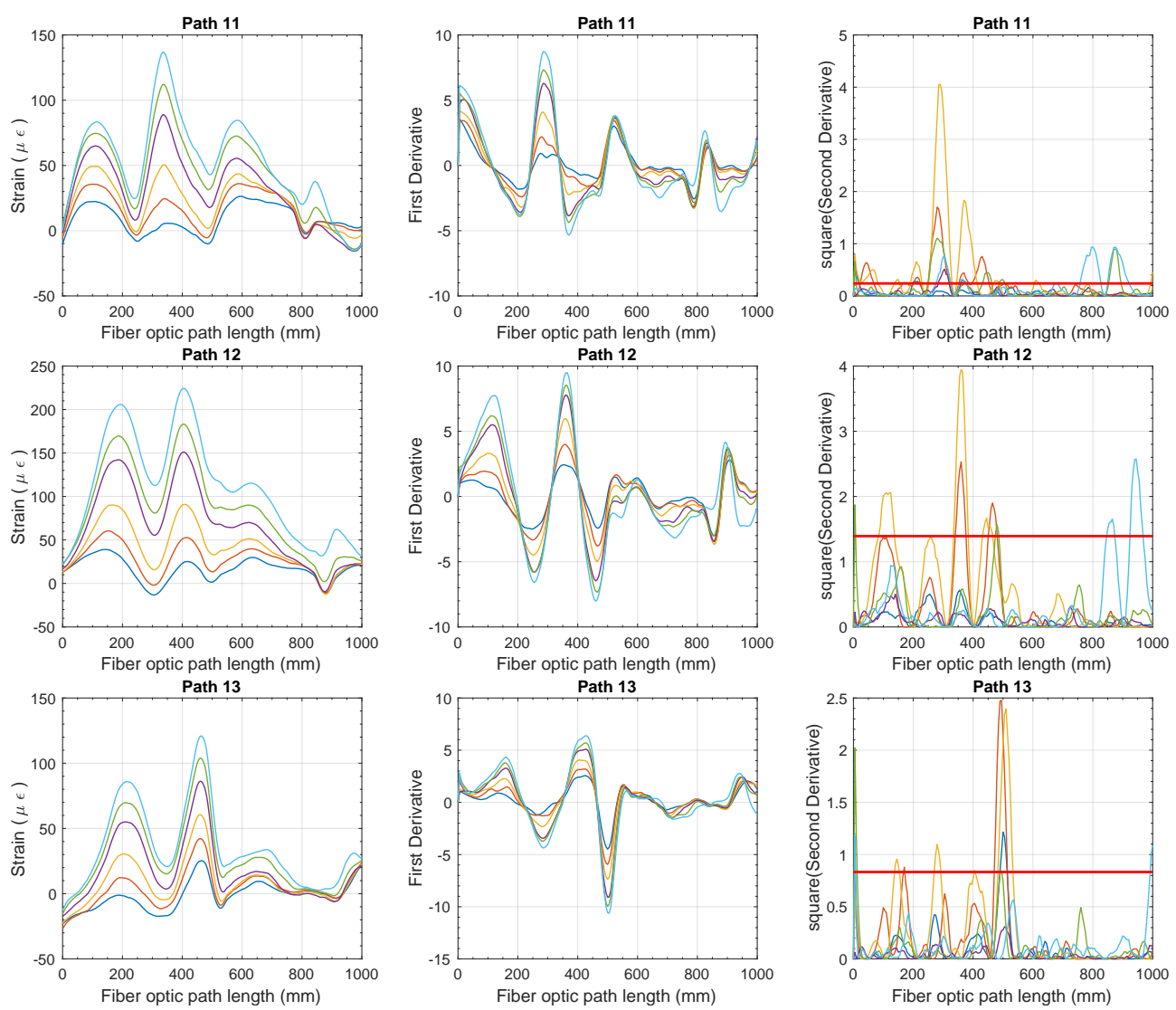

Figure 128. Results of the second derivative analysis for the stiffened panel 1. 
B. Buckling test for the stiffened panel 2.
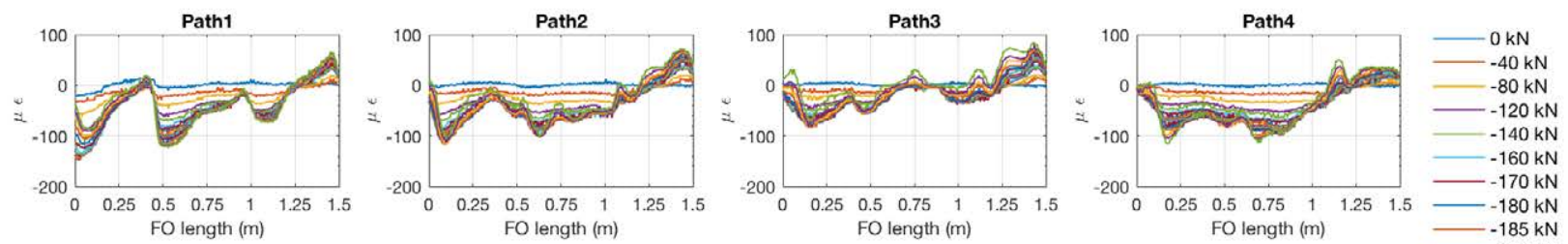

Path5

Path6
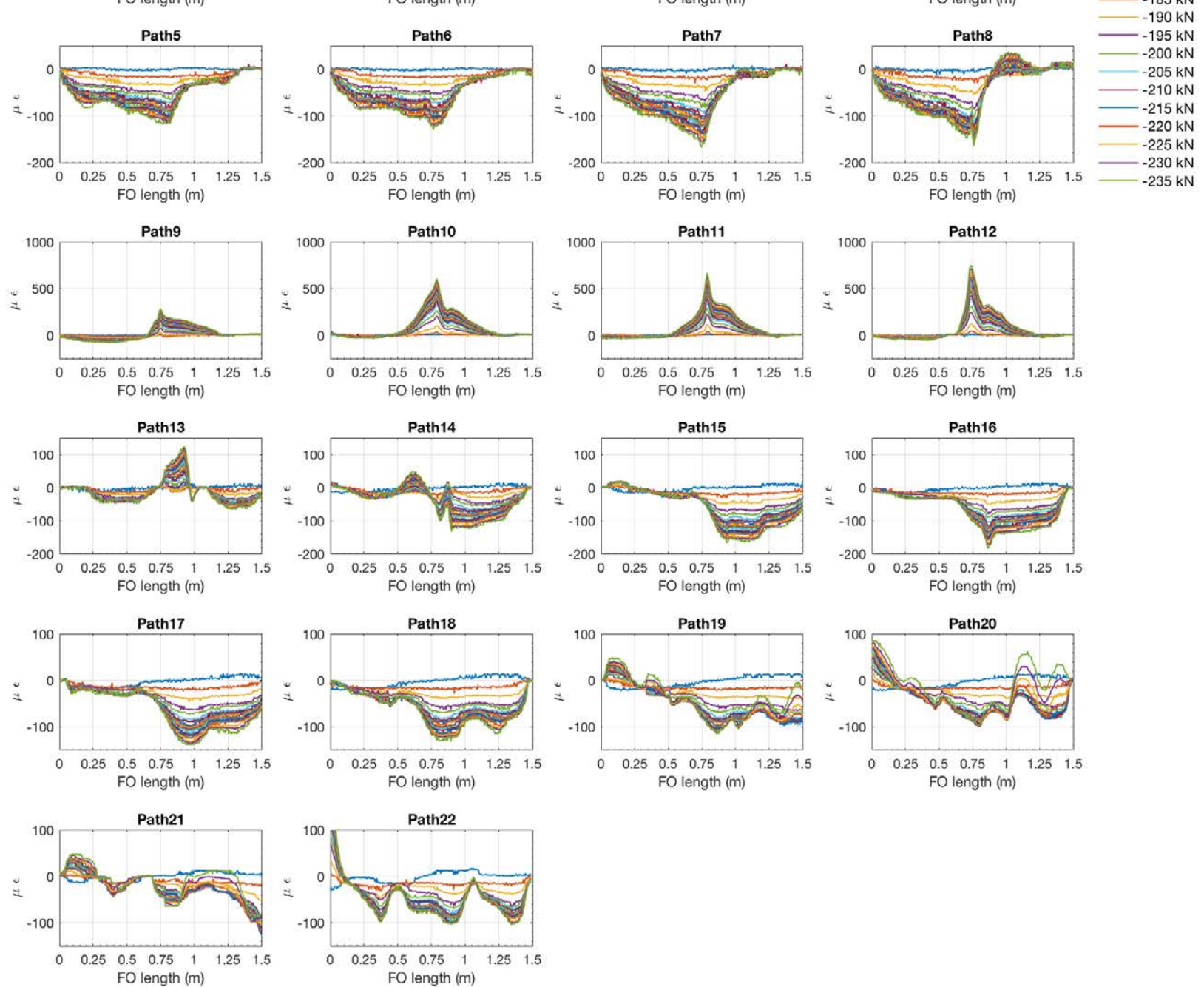

Figure 129. Whole distributed measurement for the buckling test under compressive load for the stiffened panel 2 . 

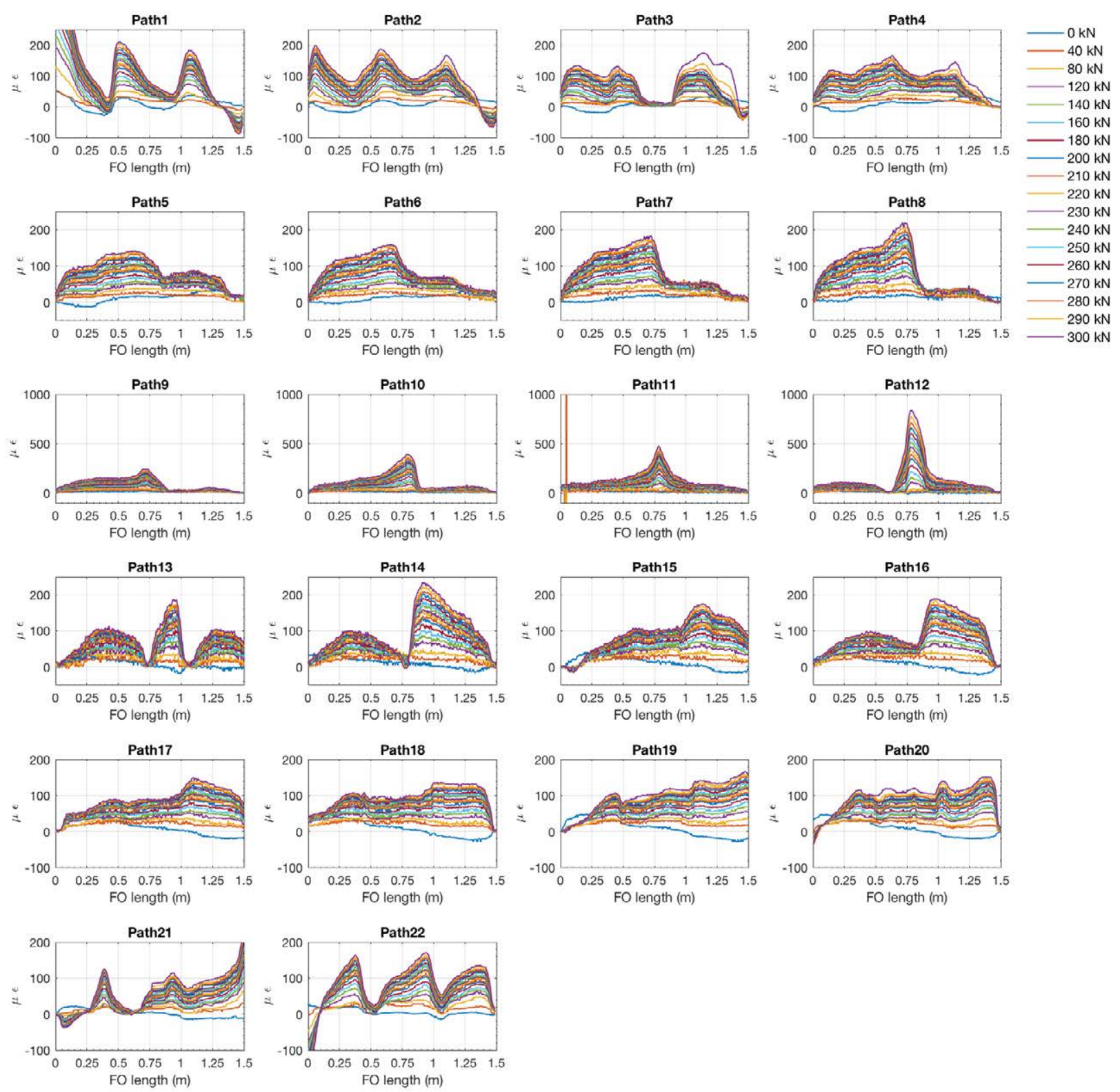

Figure 130. Whole distributed measurement for the buckling test under tensile load for the stiffened panel 2. 